\title{
Global Status and Trends in Intellectual Property Claims: Patent Dataset for Biodiversity
}

\section{PAUL OLDHAM \& ANTHONY MARK CUTTER ${ }^{1}$}

This patent dataset is made available by the authors to encourage further research and methodological development. In making the dataset available in an open access journal our aim is to encourage greater research and data sharing on intellectual property and biodiversity. On that basis the sole condition of use is attribution of authorship. Excel files are available from the authors upon request.

The research was conducted using the Advanced search function of the European Patent Office esp@ cenet "worldwide" database. ${ }^{2}$ The datasets refer to patent publication counts by publication year combined with International Patent Classification (IPC) codes. The datasets do not discriminate between patent applications and patent grants and will include re-publications as applications move through the procedure in multiple jurisdictions. As such the datasets refer to international patent activity on the systemic level. Patent documents are commonly awarded more than one IPC code and may therefore fall in more than one area of the data.

For further details on the research and data interpretation please see the accompanying article Mapping Global Status and Trends in Patent Activity for Biological and Genetic Material ${ }^{3}$ within this issue of Genomics, Society and Policy journal.

The dataset is made available as is to promote further research and consists of three sections:

\section{Search results for $2004,2005(\times 2), 2006$ (x 2), a working definition of biotechnology, and indicators for diseases (A61P)}

\section{Data comparison graphs and tables}

\section{Data coverage tables}

The authors gratefully thank CESAGen students Jodie Chapell, Catriona Forbes, Nina Moeller and Ben Soffa for their assistance in collating the datasets.

\footnotetext{
${ }^{1}$ Dr. Paul Oldham, Research Associate, ESRC Centre for Economic \& Social Aspects of Genomics (CESAGen), Lancaster University, UK (p.oldham@lancaster.ac.uk) designed and led the research. Mr. Anthony Mark Cutter, is a Barrister and Senior Lecturer, Lancashire Law School, University of Central Lancashire, UK (amcutter@uclan.ac.uk).

${ }^{2}$ http://ep.espacenet.com/advancedSearch?locale=en_EP

3 http://www.hss.ed.ac.uk/genomics/vol2no2/oldham1abstract.htm
} 


\begin{tabular}{|c|c|c|c|c|c|c|c|c|c|c|c|}
\hline & & & & 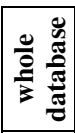 & $\underset{\substack{\infty \\
\infty \\
\infty}}{\infty}$ & $\begin{array}{l}\stackrel{a}{=} \\
\stackrel{n}{n}\end{array}$ & $\stackrel{\text { 岱 }}{-}$ & $\stackrel{n}{n}$ & & $\begin{array}{l}\tilde{b} \\
\triangleq\end{array}$ & $\begin{array}{l}8 \\
+ \\
+ \\
8 \\
8\end{array}$ \\
\hline & & 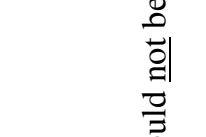 & & 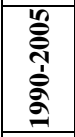 & 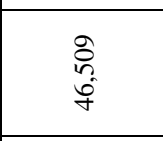 & $\stackrel{\tilde{h}}{=}$ & $\vec{\infty}$ & $\begin{array}{l}\text { 今̃ } \\
\text { in } \\
\text { in }\end{array}$ & के & $\begin{array}{l}\widehat{\infty} \\
\infty \\
\infty\end{array}$ & 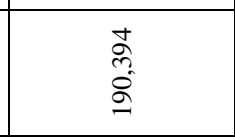 \\
\hline & & 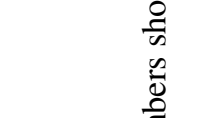 & & ลัे & $\begin{array}{l}\vec{n} \\
\stackrel{f}{f} \\
f\end{array}$ & $\stackrel{\text { ले }}{\stackrel{0}{\varrho}}$ & $\stackrel{\infty}{\curvearrowright}$ & $\begin{array}{l}\frac{4}{6} \\
\text { in }\end{array}$ & 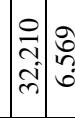 & 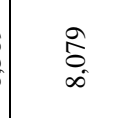 & $\begin{array}{l}\stackrel{2}{2} \\
\infty \\
\subseteq \\
\subseteq\end{array}$ \\
\hline & & $\begin{array}{l}\Xi \\
\Xi \\
0 \\
0 \\
0\end{array}$ & : & ถิ่ & 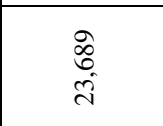 & $\begin{array}{l}0 \\
\text { d. } \\
\text { in }\end{array}$ & $\stackrel{\odot}{\ni}$ & ồ & $\begin{array}{lll}n & \infty \\
\ddots & \infty \\
\vdots & n \\
= & n & n\end{array}$ & $\begin{array}{l}\overrightarrow{\text { ते }} \\
\text { in }\end{array}$ & 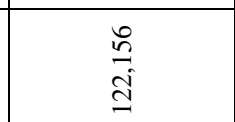 \\
\hline & & 节 & : & ڤั้ & $\stackrel{\infty}{\stackrel{\infty}{f}}$ & $\stackrel{8}{\circ}$ & $\mathbb{N}$ & $\stackrel{\infty}{\%}$ & : & $\stackrel{\infty}{\triangleright}$ & $\stackrel{\vec{B}}{=}$ \\
\hline 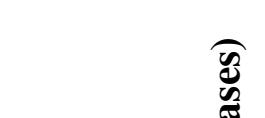 & & 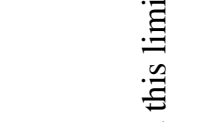 & 昰 & స్ & 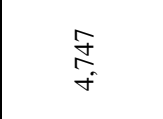 & $\begin{array}{l}\stackrel{8}{\circ} \\
\stackrel{n}{n}=\end{array}$ & $\infty$ & $\bar{m}$ & â & $\stackrel{+}{\stackrel{ \pm}{\sim}}$ & 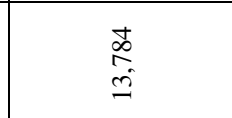 \\
\hline 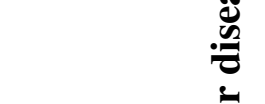 & & 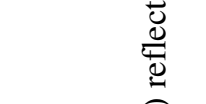 & $\begin{array}{ll}0.0 \\
0 \\
0\end{array}$ & ڤิ̀ & 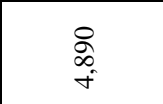 & $\stackrel{\infty}{\stackrel{\infty}{\rightarrow}}$ & $\stackrel{m}{-}$ & లె & : & $\underset{\infty}{\widetilde{N}}$ & \begin{tabular}{l}
$\infty$ \\
$\vdots$ \\
$\vdots$ \\
\multirow{J}{*}{}
\end{tabular} \\
\hline$\stackrel{\theta}{E}$ & & \begin{tabular}{l}
$\overrightarrow{0}$ \\
\multirow{4}{0}{0} \\
0.0
\end{tabular} & 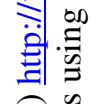 & ิิે & 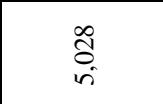 & బู & 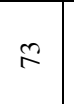 & $\stackrel{\infty}{ల}^{\infty}$ & 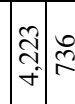 & 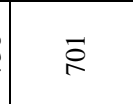 & $\begin{array}{l}\infty \\
\stackrel{0}{1} \\
\stackrel{ \pm}{ \pm}\end{array}$ \\
\hline$\stackrel{4}{\Xi}$ & & 8 & 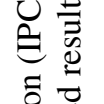 & స్తి & $\begin{array}{l}\hat{a} \\
\dot{\sigma}\end{array}$ & 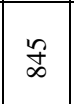 & న & ळे & लेख? & $\bar{n}$ & $\begin{array}{l}8 \\
n^{2} \\
\text { mi }\end{array}$ \\
\hline 웅 & & & 莡: & ఫ్సે & \begin{tabular}{l} 
है \\
\multirow{+}{*}{}
\end{tabular} & $\stackrel{\infty}{\infty}$ & ฉ & $\stackrel{\mathscr{\infty}}{\stackrel{\infty}{+}}$ & ले & $\frac{0}{6}$ & $\begin{array}{l} \pm \\
\infty \\
\infty \\
\end{array}$ \\
\hline 巳ٕ & & & 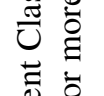 & & $\stackrel{0}{m}$ & $\underset{\infty}{ \pm}$ & 8 & $\stackrel{\wp}{\stackrel{\infty}{\sigma}}$ & ה్ & 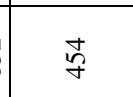 & $\begin{array}{l}\tilde{\aleph} \\
\text { త్ }\end{array}$ \\
\hline$\stackrel{\circ}{\circ}$ & & & ᄅ. & $\stackrel{\circ}{\circ}$ & 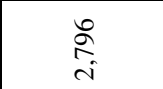 & $\stackrel{\sim}{\sim}$ & $\infty$ & ঙ̊গ్ & 今̂n & 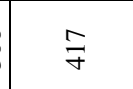 & 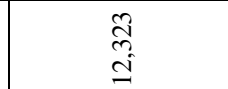 \\
\hline อี & & 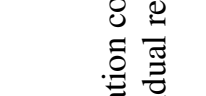 & 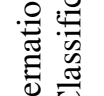 & $\hat{\sigma}$ & $\frac{\bar{a}}{i}$ & $\stackrel{8}{8}$ & $\stackrel{\infty}{\sim}$ & $\stackrel{\infty}{\sim}$ & 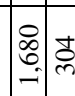 & \&্+ & $\stackrel{\Xi}{\exists}$ \\
\hline 递 & & 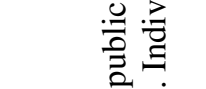 & 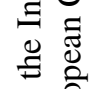 & $\stackrel{\circ}{\circ}$ & $\stackrel{n}{a}$ & 奋 & $\tilde{\mathrm{N}}$ & $\stackrel{\Rightarrow}{m}$ & 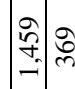 & $\bar{F}$ & 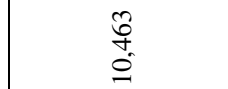 \\
\hline$\stackrel{\infty}{30}$ & & & 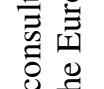 & $\stackrel{\circ}{\circ}$ & 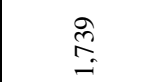 & $\underset{ల}{\infty}$ & $\stackrel{0}{0}$ & $\stackrel{0}{d}$ & (ָ) & 字 & 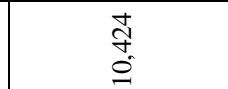 \\
\hline 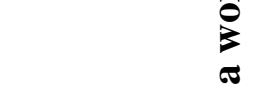 & & & 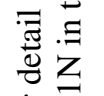 & 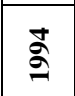 & $\begin{array}{l}\overrightarrow{\sigma_{0}} \\
-\end{array}$ & fo & 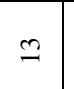 & ڤे & ڤ్రి & $\stackrel{\dddot{q}}{q}$ & $\stackrel{\infty}{=}$ \\
\hline ठั้ & & 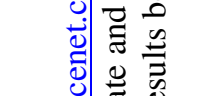 & 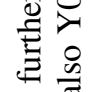 & ڤొ & $\stackrel{\widehat{B}}{-}$ & ஓొ & $\vec{\sim}$ & $\vec{d}$ & $\stackrel{\infty}{=}$ & $\vec{q}$ & 亭 \\
\hline$\sqrt{2}$ & & 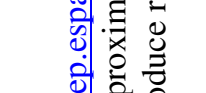 & 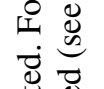 & $\tilde{\Xi}$ & $\stackrel{\vec{n}}{n}$ & 寻 & $\hat{\sim}$ & $\frac{\pi}{m}$ & $\begin{array}{l}a \\
\infty\end{array}$ & 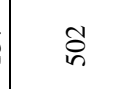 & 㤐 \\
\hline త్ & $\underset{7}{8}$ & 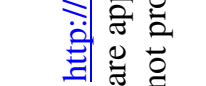 & 㞼 & $\bar{\sigma}$ & 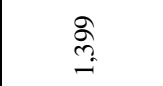 & $\stackrel{\infty}{\infty}$ & $\ddot{\sim}$ & ह & $\underset{\infty}{\infty} \mid \equiv$ & ஜु & 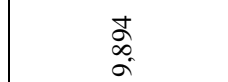 \\
\hline ஓ્ণ & లై & 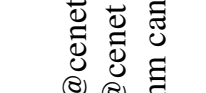 & 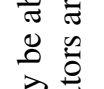 & Бิ & $\stackrel{\Xi}{\stackrel{I}{\leftrightarrows}}$ & ది & $\ddot{m}$ & $\stackrel{\sim}{\sim}$ & : & $\stackrel{\sim}{n}$ & $\begin{array}{l}\infty \\
2 \\
\alpha\end{array}$ \\
\hline 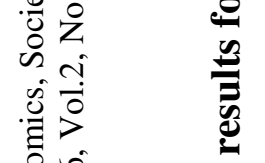 & 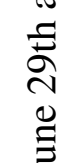 & 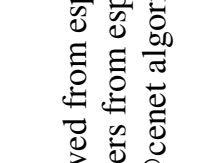 & 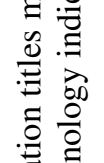 & $\stackrel{\varrho}{\varrho}$ & $\frac{\pi}{0}$ & 瞇 & 愛 & 龺 & 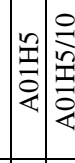 & $\begin{array}{l}\overrightarrow{0} \\
\stackrel{3}{0}\end{array}$ & $\frac{z}{a}$ \\
\hline 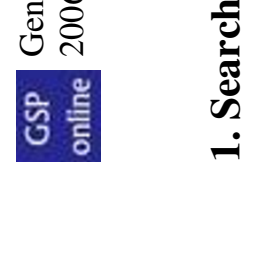 & : & 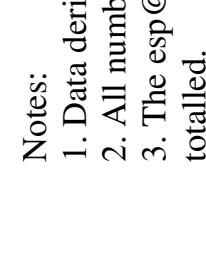 & 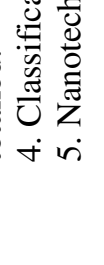 & 言 & 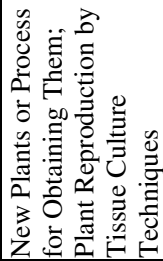 & 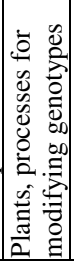 & 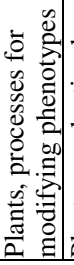 & 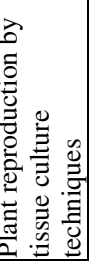 & 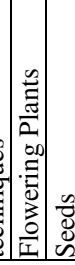 & 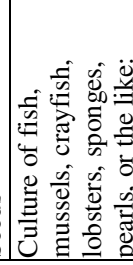 & 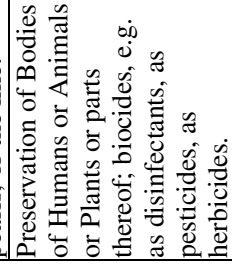 \\
\hline
\end{tabular}




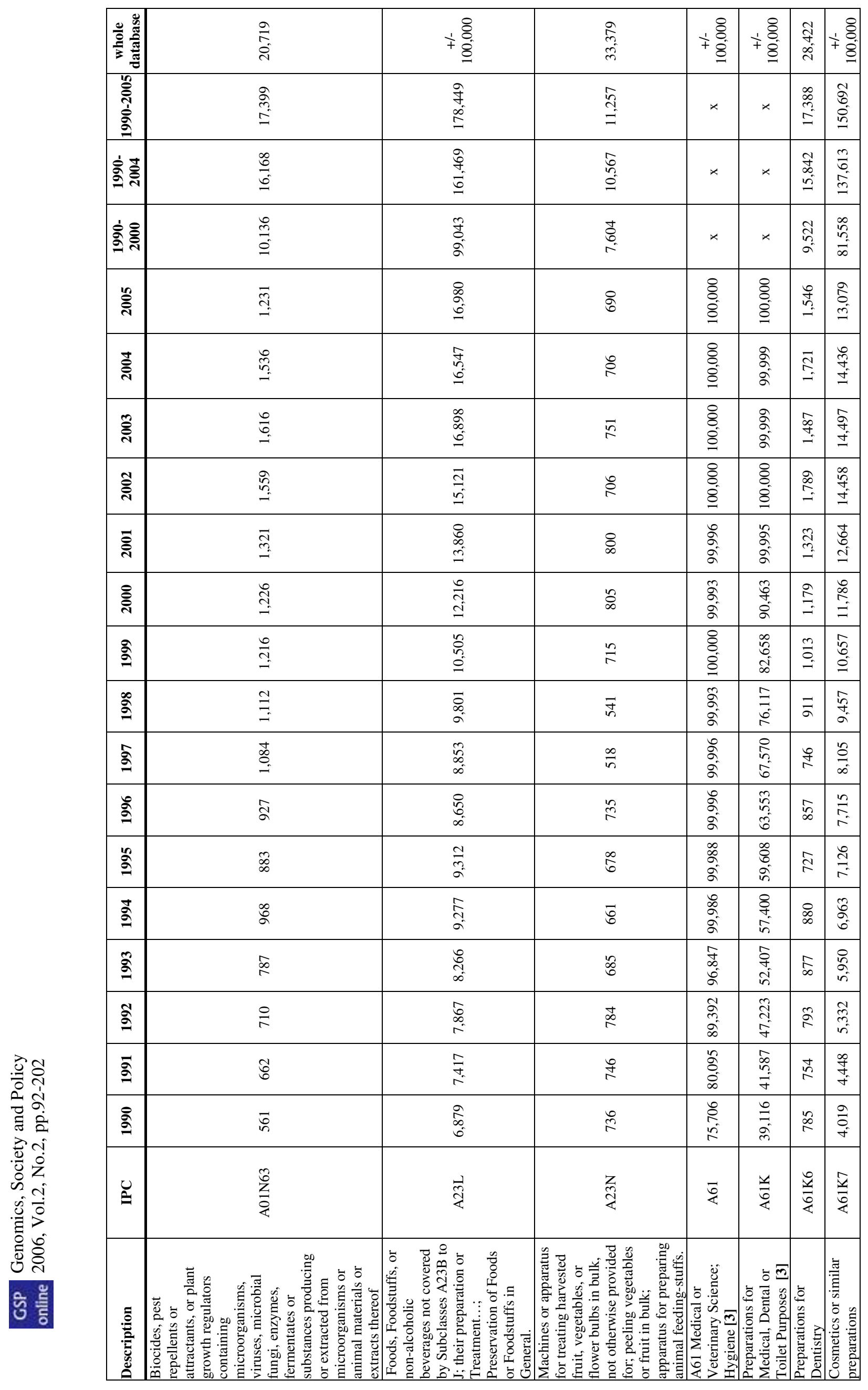




\begin{tabular}{|c|c|c|c|c|c|c|c|c|c|c|c|c|c|}
\hline & $\begin{array}{l}\text { ro } \\
\text { d. } \\
\end{array}$ & 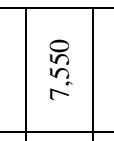 & 总 & 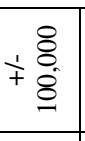 & 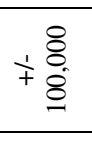 & & & & & 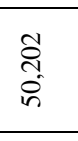 & 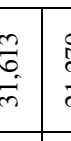 & & \\
\hline & 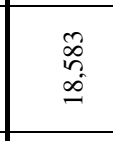 & 暿 & $\frac{m}{\bar{s}}$ & 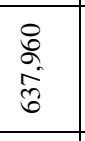 & 高 & & & & 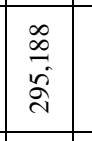 & 嘉 & 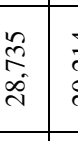 & & \\
\hline & 胥 & 喜 & 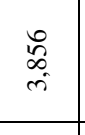 & 总 & $\begin{array}{l}\overline{\bar{g}} \\
\bar{d} \\
\bar{g}\end{array}$ & & & & 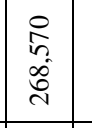 & 墨 & & & \\
\hline & हू & 売 & $\stackrel{\circ}{0}$ & స్ & 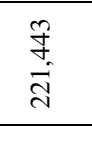 & & 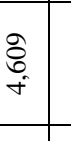 & & $\mid$ & 啇 & 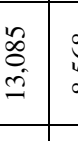 & & \\
\hline & 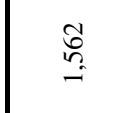 & 8 & 5 & 売 & $\frac{8}{p}$ & त & 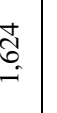 & & $\mid$ & $\begin{array}{c}n \\
c \\
c \\
c\end{array}$ & $\begin{array}{l}\text { in } \\
\text { in }\end{array}$ & & \\
\hline & 虽 & 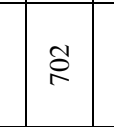 & f & 劲 & 高 & 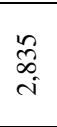 & 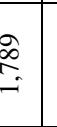 & & $\mid$ & ְ̊g & 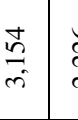 & & \\
\hline & 嘉 & $\frac{a}{x}$ & 筈 & 等 & 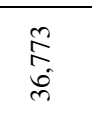 & Fु & 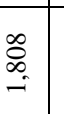 & 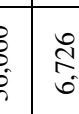 & 总 & $\frac{\infty}{\infty}$ & 焗 & & \\
\hline & క్ & $\bar{\alpha}$ & 票 & 营 & 䋧 & $y_{j}^{2}$ & 虽 & 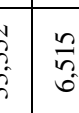 & 喆 & 素 & कृ & & 童 \\
\hline & $\frac{n}{n g}$ & 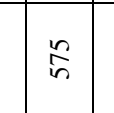 & S & 紊 & 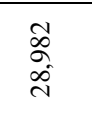 & $=$ & 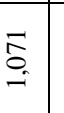 & 敌 & 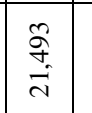 & 然 & ङ & & $\overbrace{\infty}^{\infty}$ \\
\hline & ఫ్ త్ & 7 & $\stackrel{x}{*}$ & 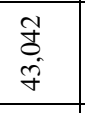 & 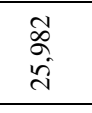 & $\stackrel{3}{=}$ & $\frac{1}{\infty}$ & $\begin{array}{l}9 \\
7 \\
n \\
n\end{array}$ & 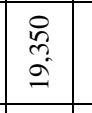 & $\frac{⿱ 0}{d}$ & $\frac{8}{\pi}$ & & 胥 \\
\hline & $\stackrel{g}{2}$ & 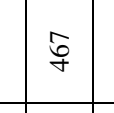 & 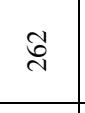 & 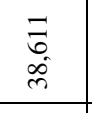 & 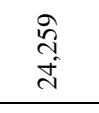 & $\stackrel{\circ}{g}$ & 8 & $\frac{a}{b}$ & 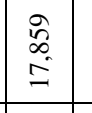 & $\frac{\mathrm{o}}{\mathrm{N}}$ & 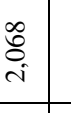 & & $\frac{5}{8}$ \\
\hline 2 & 军 & 呆 & त्व & 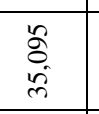 & 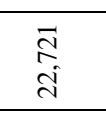 & 总 & $\overline{\bar{n}}$ & $\frac{8}{7}$ & 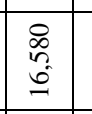 & तี & 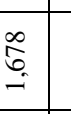 & & 䨖 \\
\hline & 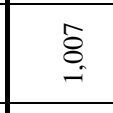 & \begin{tabular}{|c|} 
g \\
\end{tabular} & $\stackrel{\square}{\square}$ & $\begin{array}{l}\text { 总 } \\
\bar{d}\end{array}$ & $\begin{array}{l}\frac{\mathrm{a}}{\mathrm{g}} \\
\end{array}$ & $\stackrel{8}{\circ}$ & 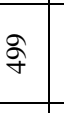 & 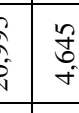 & \begin{tabular}{|l|}
$\overrightarrow{\bar{n}}$ \\
$\underline{\underline{b}}$
\end{tabular} & สู & 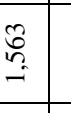 & & 5 \\
\hline S & $\stackrel{\mathrm{g}}{\mathrm{g}}$ & 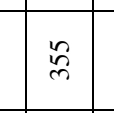 & : & 号 & 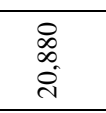 & $\bar{\otimes}$ & 京 & 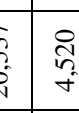 & 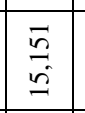 & 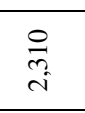 & 噌 & & 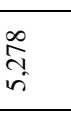 \\
\hline & ने & $\stackrel{ \pm}{m}$ & I & $\frac{\mathrm{g}}{0}$ & 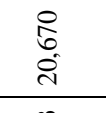 & 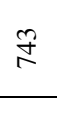 & 哭 & $\frac{1}{3}$ & 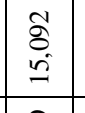 & 悹 & : & & 总 \\
\hline 8 & 亏 & $\ddot{m}$ & $\stackrel{\square}{2}$ & 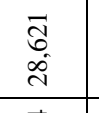 & $\frac{\pi}{8}$ & $\stackrel{8}{8}$ & $\stackrel{\circ}{\circ}$ & 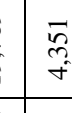 & 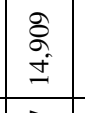 & $\stackrel{m}{i}_{i}^{\circ}$ & $\frac{2}{2}$ & & $\begin{array}{l}\text { fo } \\
z\end{array}$ \\
\hline & $\frac{2}{2}$ & $\approx$ & $\overrightarrow{\underline{a}}$ & 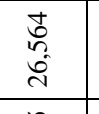 & 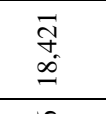 & 8 & : & & 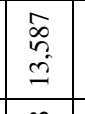 & $\overline{\bar{g}}$ & : & & 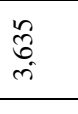 \\
\hline . & 吉 & \pm & $\therefore$ & 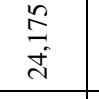 & 葛 & 啹 & 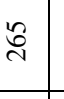 & & $\begin{array}{l}9 \\
\text { If }\end{array}$ & $\stackrel{\text { of }}{z=1}$ & 5 & & $\bar{d} \frac{\pi}{2}$ \\
\hline 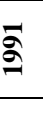 & $\check{n}$ & $\overline{2}$ & +8 & $\begin{array}{l}\frac{\infty}{q} \\
\substack{a \\
c}\end{array}$ & 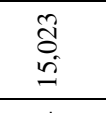 & $\stackrel{\circ}{q}$ & 部 & & 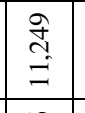 & 总 & $\stackrel{\leftrightarrow}{\mathrm{g}}$ & & $\bar{్}$ \\
\hline & ş & $\cong$ & $\therefore$ & 浩 & 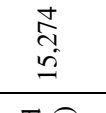 & & & & $\begin{array}{l}\text { 总 } \\
\stackrel{0}{=}\end{array}$ & 疍 & $\frac{7}{7}$ & & 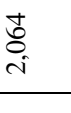 \\
\hline & & & $\begin{array}{l}\frac{\bar{x}}{2} \\
\frac{3}{2}\end{array}$ & $\frac{\overrightarrow{3}}{\underline{\underline{y}}}$ & 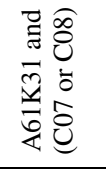 & & & & & 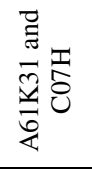 & & & 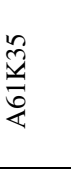 \\
\hline
\end{tabular}




\begin{tabular}{|c|c|c|c|c|c|c|c|c|c|c|c|c|c|c|c|c|}
\hline 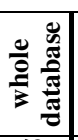 & $\begin{array}{l}n \\
\hat{b} \\
f \\
f\end{array}$ & $\begin{array}{c}\vec{f} \\
\underset{c}{\infty} \\
i\end{array}$ & 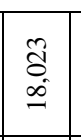 & $\begin{array}{l}n \\
a \\
a \\
\dot{\lambda}\end{array}$ & $\mid \begin{array}{c}0 \\
0 \\
0 \\
0 \\
0\end{array}$ & 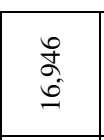 & 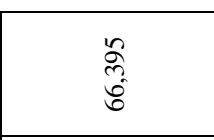 & $\begin{array}{l}\overrightarrow{\widehat{O}} \\
\stackrel{-}{\Omega}\end{array}$ & 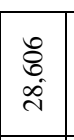 & $\begin{array}{l}\hat{\text { ̀े }} \\
\text { in }\end{array}$ & $\stackrel{\leftrightarrow}{\circ}$ & $\stackrel{5}{=}$ & $\stackrel{+}{\stackrel{n}{-}}$ & $\left|\begin{array}{r|r} & 8 \\
1 & 8 \\
+ & 0 \\
& 0\end{array}\right|$ & $\begin{array}{r}8 \\
+ \\
+ \\
+ \\
0\end{array}$ & 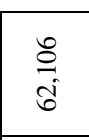 \\
\hline 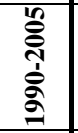 & $\begin{array}{l} \pm \\
\text { ¿े } \\
\text { बे }\end{array}$ & $\begin{array}{l}\hat{\alpha} \\
\dot{\alpha}\end{array}$ & 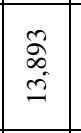 & 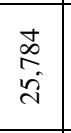 & $\begin{array}{l}\stackrel{2}{R} \\
\text { a }\end{array}$ & $\begin{array}{l}\text { an } \\
\text { nn } \\
\end{array}$ & $\begin{array}{l}\infty \\
i n \\
\vdots \\
0 \\
0\end{array}$ & $\frac{9}{a}$ & 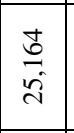 & 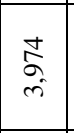 & $\stackrel{\text { શे }}{\rightarrow}$ & $\stackrel{\infty}{\infty}$ & $\stackrel{\infty}{\stackrel{\infty}{-}}$ & 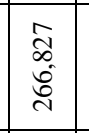 & 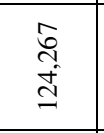 & $\begin{array}{l}\text { ठे } \\
\text { in }\end{array}$ \\
\hline 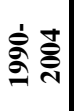 & $\begin{array}{l}0 \\
= \\
0\end{array}$ & $\begin{array}{c}\overrightarrow{\sigma_{0}} \\
\stackrel{\infty}{\infty}\end{array}$ & $\begin{array}{l}\text { Cू } \\
\text { ปे }\end{array}$ & $\begin{array}{l}\infty \\
\text { ते } \\
\underset{\sim}{3}\end{array}$ & $\begin{array}{l}\vec{d} \\
\infty \\
\infty \\
\infty\end{array}$ & 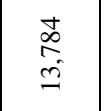 & $\frac{8}{i}$ & 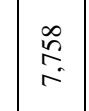 & $\begin{array}{l}\infty \\
8 \\
0 \\
2\end{array}$ & $\begin{array}{c}\tilde{N} \\
\tilde{n} \\
m\end{array}$ & $\begin{array}{l}\overrightarrow{\mathrm{D}} \\
\stackrel{\mathrm{N}}{二}\end{array}$ & 早 & $\stackrel{\stackrel{ }{二}}{=}$ & 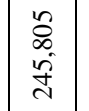 & $\begin{array}{l}\underset{\Xi}{\Xi} \\
\cong\end{array}$ & हे \\
\hline डેे & $\begin{array}{l}0 \\
\stackrel{0}{0} \\
i\end{array}$ & $\begin{array}{l}\stackrel{c}{o} \\
\stackrel{m}{=} \\
=\end{array}$ & 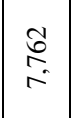 & $\stackrel{\infty}{\stackrel{\infty}{=}}$ & $\begin{array}{l}\text { gi } \\
\stackrel{+}{+}\end{array}$ & $\begin{array}{l}0 \\
0 \\
0.0 \\
0 .\end{array}$ & $\begin{array}{l}\text { 今̂ } \\
\infty \\
\text { ci }\end{array}$ & 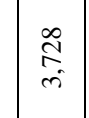 & $\underset{\substack{\circ \\
\rightarrow}}{\stackrel{0}{-}}$ & 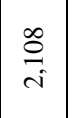 & $\stackrel{\sim}{\approx}$ & $\tilde{\Im}$ & $\frac{\partial}{6}$ & $\underset{\substack{y \\
\stackrel{5}{2}}}{\stackrel{y}{a}}$ & $\begin{array}{l}\overrightarrow{\mathrm{n}} \\
\overrightarrow{6}\end{array}$ & $\begin{array}{l}\text { है } \\
\text { ì }\end{array}$ \\
\hline ڤ్̀ે & $\begin{array}{l}\infty \\
\infty \\
\infty \\
\infty\end{array}$ & 号 & $\bar{\sigma}$ & $\begin{array}{l}0 \\
n \\
i \\
i\end{array}$ & $\ddot{\alpha}$ & $\stackrel{\vec{f}}{\stackrel{7}{n}}$ & $\begin{array}{l}\stackrel{\infty}{f} \\
\stackrel{\sigma}{\sigma}\end{array}$ & $\underset{\substack{\infty \\
=}}{=}$ & $\begin{array}{l}0 \\
6 \\
6 \\
6\end{array}$ & $\tilde{\sigma}$ & $\stackrel{\infty}{=}$ & 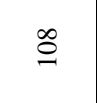 & $\stackrel{\nearrow}{\beth}$ & $\begin{array}{l}\tilde{\delta} \\
\vec{d}\end{array}$ & 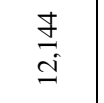 & $\frac{m}{m_{0}}$ \\
\hline ذ్ڤે & 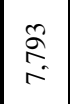 & 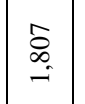 & $\underset{\widetilde{N}}{-}$ & 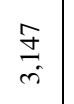 & $\stackrel{\infty}{\stackrel{0}{=}}$ & $\stackrel{f}{\stackrel{F}{2}}$ & $\begin{array}{c}\vec{\infty} \\
\infty \\
0\end{array}$ & $\underset{\mathbb{Z}}{\mathbb{Z}}$ & $\begin{array}{c}+ \\
\stackrel{+}{\infty} \\
\stackrel{+}{+}\end{array}$ & bे & $\stackrel{ \pm}{=}$ & t & 品 & 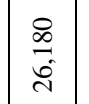 & 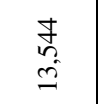 & 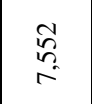 \\
\hline ڤิ̀ & 寺 & $\begin{array}{l}\tilde{O} \\
i \\
i\end{array}$ & $\stackrel{\vec{g}}{-}$ & $\underset{\vec{g}}{\vec{r}}$ & $\stackrel{2}{\stackrel{2}{-}}$ & 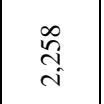 & $\stackrel{\infty}{\stackrel{\infty}{\sim}}$ & $\stackrel{m}{=}$ & $\begin{array}{l}\overrightarrow{0} \\
\infty \\
+\end{array}$ & $\vec{q}$ & $\widehat{\Xi}$ & $\Xi$ & $\stackrel{\triangleright}{\stackrel{\infty}{-}}$ & 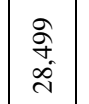 & $\begin{array}{l}n \\
\tilde{n} \\
n \\
n\end{array}$ & $\underset{\infty}{\infty}$ \\
\hline ธ్ิ & $\begin{array}{l}\stackrel{D}{\circ} \\
\text { o. }\end{array}$ & $\stackrel{\widetilde{\sigma}}{\sigma}$ & 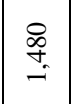 & $\begin{array}{l}n \\
\stackrel{n}{m} \\
\dot{m}\end{array}$ & $\stackrel{\vartheta}{\stackrel{m}{二}}$ & ơ & $\begin{array}{l}n \\
n \\
0\end{array}$ & no & \begin{tabular}{l}
$\stackrel{0}{ }$ \\
\multirow{2}{*}{} \\
$\forall$
\end{tabular} & $\vec{n}$ & సे & in & $\stackrel{\leftrightarrow}{0}$ & 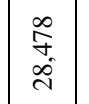 & $\begin{array}{l}\stackrel{n}{a} \\
\stackrel{-}{n}\end{array}$ & $\underset{\infty}{\stackrel{d}{d}}$ \\
\hline క్తి & $\begin{array}{l}\stackrel{\sigma}{\infty} \\
\underset{+}{+}\end{array}$ & 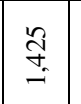 & $\underset{f}{\stackrel{f}{g}}$ & 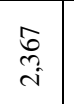 & 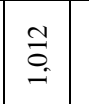 & $\stackrel{\vec{n}}{=}$ & $\begin{array}{l}\stackrel{0}{0} \\
+ \\
+\end{array}$ & $\stackrel{m}{2}$ & बे & ్ֶ & $\approx$ & $\stackrel{t}{n}$ & a & $\frac{8}{3}$ & $\begin{array}{l}\stackrel{\infty}{\neq} \\
\stackrel{0}{\subseteq}\end{array}$ & 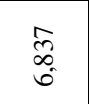 \\
\hline క్ેి & 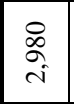 & $\begin{array}{l}\text { o } \\
\stackrel{\sim}{-} \\
-\end{array}$ & డ & $\begin{array}{c}\sigma \\
\stackrel{-}{\circ} \\
-\end{array}$ & ळे & $\stackrel{\Xi}{\cong}$ & $\begin{array}{l}\text { के } \\
\text { ले } \\
\text {. }\end{array}$ & 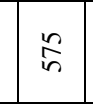 & 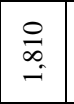 & $\stackrel{\sim}{\sim}$ & 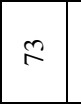 & $\stackrel{\infty}{+}$ & $\cong$ & $\begin{array}{l}\text { î } \\
\text { î }\end{array}$ & $\vec{\sigma}$ & $\begin{array}{l}0 \\
\text { do } \\
\text { in }\end{array}$ \\
\hline बे & * & $\begin{array}{l}\mathfrak{o} \\
\stackrel{\sim}{-}\end{array}$ & $\stackrel{\circ}{\circ}$ & 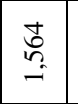 & 8 & $\stackrel{\infty}{\circ}$ & $\underset{\stackrel{R}{f}}{\stackrel{p}{m}}$ & $\stackrel{\vec{n}}{n}$ & $\star$ & 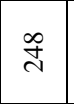 & $\cong$ & if & $\vec{n}$ & $\begin{array}{l}\stackrel{\infty}{\infty} \\
\stackrel{2}{2}\end{array}$ & $\frac{m}{n}$ & $\begin{array}{l}\stackrel{\infty}{\infty} \\
\stackrel{+}{+}\end{array}$ \\
\hline$\stackrel{\infty}{\partial}$ & $\star$ & $\stackrel{\partial}{\leftrightarrows}$ & $\tilde{\infty}$ & 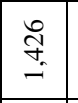 & $\stackrel{0}{i n}$ & స్రి & $\stackrel{m}{m}$ & 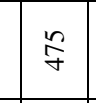 & $\star$ & $\underset{\sim}{\sim}$ & $\infty$ & $\ddot{m}$ & 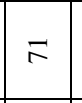 & $\begin{array}{l}\bar{y} \\
\infty \\
\stackrel{\sigma}{a}\end{array}$ & $\stackrel{5}{\stackrel{2}{2}}$ & 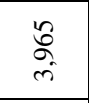 \\
\hline$\hat{\sigma}$ & $\star$ & $\begin{array}{l}n \\
\stackrel{2}{=} \\
=\end{array}$ & $\bar{\infty}$ & 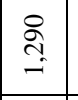 & $\underset{⿱}{\vec{G}}$ & $\stackrel{n}{\sim}$ & $\stackrel{\equiv}{\equiv}$ & $\hat{g}$ & $\star$ & 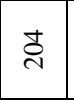 & 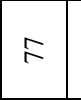 & $\stackrel{\infty}{m}$ & $\nsubseteq$ & $\begin{array}{l}\frac{\infty}{m} \\
\underline{n}\end{array}$ & 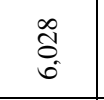 & 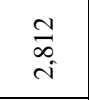 \\
\hline పे & $\star x$ & $\stackrel{n}{=}$ & $\overbrace{\infty}$ & $\stackrel{\Xi}{=}$ & f & $\bar{\delta}$ & $\begin{array}{l}\hat{E} \\
\text { i }\end{array}$ & 尺) & $\star$ & ڤి & 8 & $\mathscr{F}$ & 8 & $\begin{array}{l}\text { సे } \\
\text { ळे }\end{array}$ & $\begin{array}{l}0 \\
b \\
b \\
b \\
\end{array}$ & $\begin{array}{l}\text { ta } \\
\text { i } \\
\end{array}$ \\
\hline$\stackrel{\circ}{\circ}$ & $\star$ & $\stackrel{\mathfrak{g}}{=}$ & F & $\stackrel{\circ}{\swarrow}$ & હે & \& & $\begin{array}{l}\hat{6} \\
\text { i }\end{array}$ & f & $\star$ & $\stackrel{2}{9}$ & $\stackrel{\circ}{\circ}$ & q & $\tilde{n}$ & $\begin{array}{l}+ \\
\stackrel{0}{\circ} \\
\stackrel{\Xi}{=}\end{array}$ & $\begin{array}{l}n \\
\text { s. } \\
i \\
i n\end{array}$ & $\stackrel{9}{=}$ \\
\hline I & $\star$ & $\tilde{\tilde{\delta}}$ & 导 & $\hat{\infty}_{\infty}$ & $\stackrel{\infty}{\infty} \underset{\sim}{\infty}$ & $\stackrel{n}{q}$ & $\begin{array}{l}\text { đั } \\
\text { i }\end{array}$ & $\vec{d}$ & $\star$ & $\Xi$ & in & i & in & 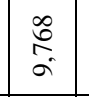 & $\begin{array}{l}\text { P } \\
\text { in }\end{array}$ & $\Sigma$ \\
\hline$\stackrel{\partial}{\sigma}$ & $x$ & ลू & 舟 & $\stackrel{\circ}{0}$ & ڤે & $\hat{m}$ & $\stackrel{m}{\underline{G}}$ & ন & $\star$ & $\stackrel{\infty}{\infty}$ & $\hat{n}$ & $\bar{m}$ & q & 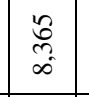 & $\begin{array}{l}\stackrel{\sqrt{n}}{ } \\
\text { + }\end{array}$ & के \\
\hline$\tilde{\sigma}$ & $\star$ & $\frac{n}{\infty}$ & 胥 & $\tilde{n}$ & $\stackrel{\infty}{ \pm}$ & ֶે & ले & $\stackrel{2}{2}$ & $\star$ & I & $\stackrel{i}{n}$ & $\hat{n}$ & F & 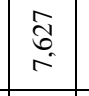 & $\begin{array}{l}\hat{S} \\
\stackrel{+}{*}\end{array}$ & in \\
\hline$\overline{\hat{\sigma}}$ & $\star$ & గి & ণ̊ & $\hat{\vec{\gamma}}$ & 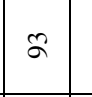 & $\stackrel{œ}{2}$ & $\stackrel{\infty}{\circ}$ & $\tilde{n}$ & $\star$ & $\underset{J}{ \pm}$ & $q$ & $m$ & $m$ & 点 & $\begin{array}{l}\infty \\
\stackrel{\infty}{m} \\
\dot{m}\end{array}$ & $\stackrel{\infty}{n}$ \\
\hline ڤ్ & $\star$ & $\frac{\pi}{6}$ & 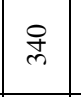 & $\hat{m}$ & in & $\stackrel{\circ}{n}$ & $\stackrel{5}{\circ}$ & $\cong$ & $\star$ & $\stackrel{n}{n}$ & in & ల & 寸 & $\begin{array}{l}n \\
\hat{n} \\
n \\
n\end{array}$ & $\underset{m}{\stackrel{\overbrace{}}{m}}$ & $\approx$ \\
\hline$\stackrel{\varrho}{\Xi}$ & 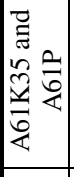 & 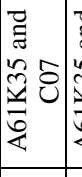 & 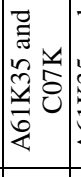 & 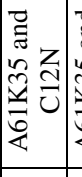 & 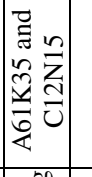 & 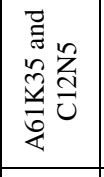 & 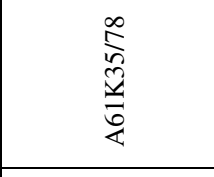 & 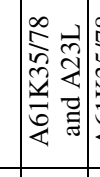 & 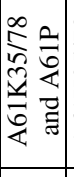 & 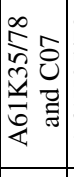 & 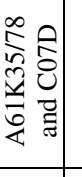 & 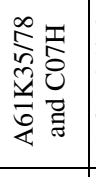 & 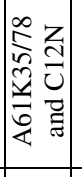 & 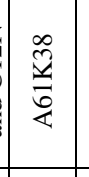 & $\frac{\widehat{\vec{d}}}{\overrightarrow{0}}$ & $\frac{\infty}{d}$ \\
\hline 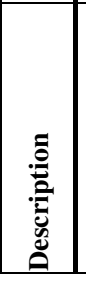 & 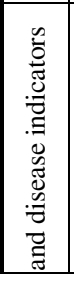 & 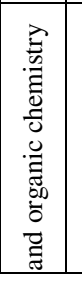 & 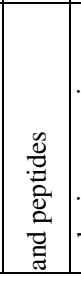 & 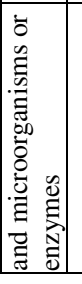 & 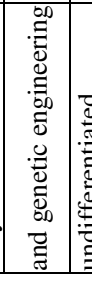 & 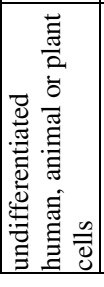 & 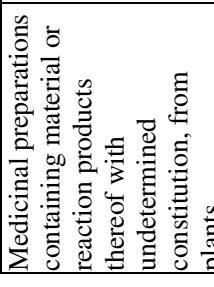 & 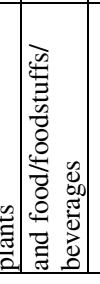 & 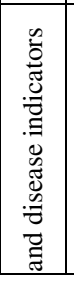 & 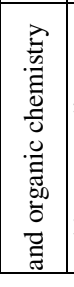 & 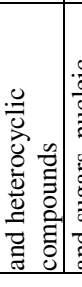 & 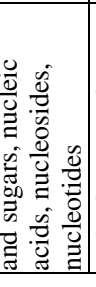 & 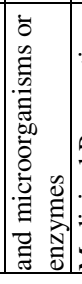 & 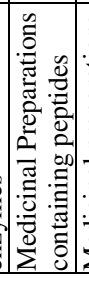 & 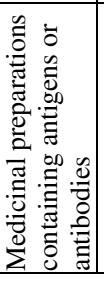 & 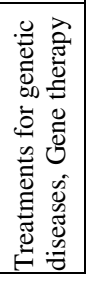 \\
\hline
\end{tabular}




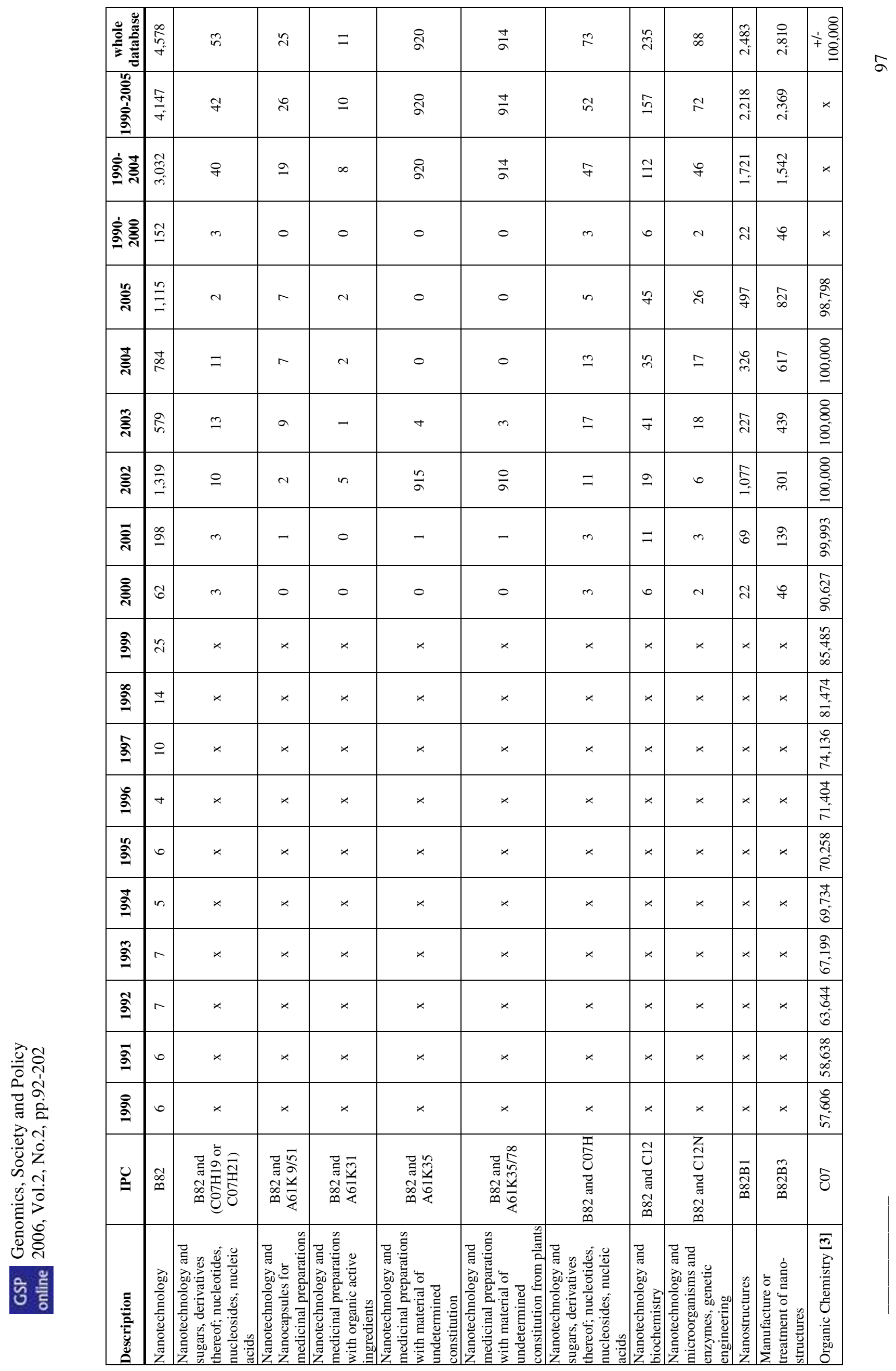




\begin{tabular}{|c|c|c|c|c|c|c|c|c|c|c|c|}
\hline 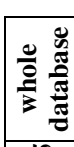 & & & 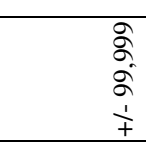 & 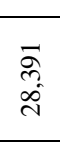 & $\begin{array}{r}\quad 8 \\
1 \\
+ \\
+ \\
0\end{array}$ & $\begin{array}{l}\infty \\
\infty \\
\infty \\
\infty \\
\end{array}$ & \begin{tabular}{r}
1 \\
1 \\
+ \\
+ \\
\hdashline \\
\hdashline \\
0
\end{tabular} & 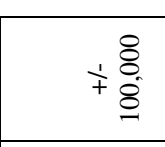 & $\begin{array}{l}\infty \\
0 \\
0 \\
0 \\
0\end{array}$ & 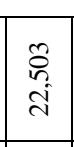 & 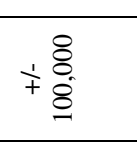 \\
\hline 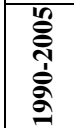 & 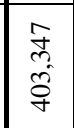 & 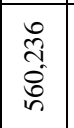 & 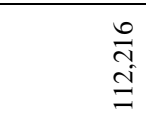 & $\begin{array}{l}\underset{\Delta}{\Delta} \\
+ \\
+\end{array}$ & 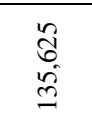 & 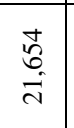 & \begin{tabular}{|l|} 
\\
$\vdots$ \\
d. \\
\end{tabular} & $\begin{array}{l}n \\
\vdots \\
\bar{\infty} \\
\bar{\infty}\end{array}$ & $\frac{\sqrt{\mathrm{m}}}{\mathrm{m}}$ & $\begin{array}{l}0 \\
\hat{n} \\
0 \\
0\end{array}$ & 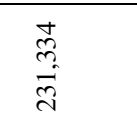 \\
\hline 言 & \begin{tabular}{|l|} 
\\
$\infty$ \\
$\infty$ \\
$\infty$ \\
$\infty$ \\
$m$
\end{tabular} & 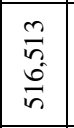 & 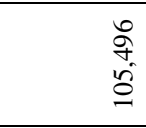 & 点 & $\begin{array}{l}\frac{\infty}{n} \\
\frac{a}{d} \\
d\end{array}$ & 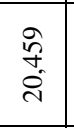 & \begin{tabular}{|l|}
$n$ \\
0 \\
0 \\
$n$ \\
$m$ \\
$m$
\end{tabular} & 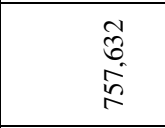 & 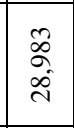 & $\begin{array}{l}\infty \\
\stackrel{\infty}{0} \\
0\end{array}$ & 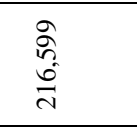 \\
\hline 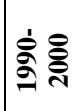 & 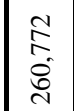 & $\begin{array}{l}\hat{s} \\
\hat{s} \\
\text { d. }\end{array}$ & 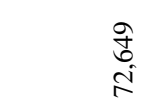 & గ్రి & 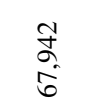 & 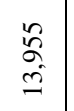 & \begin{tabular}{|l|}
\multirow{2}{*}{} \\
$\stackrel{2}{2}$ \\
$\stackrel{2}{2}$
\end{tabular} & 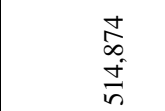 & $\begin{array}{l}\text { త్ర } \\
\stackrel{2}{2}\end{array}$ & $\frac{n}{7}$ & $\begin{array}{l}\infty \\
\stackrel{0}{0} \\
\substack{0 \\
త}\end{array}$ \\
\hline 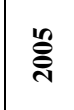 & 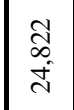 & त̂ & ఫิ & $\Xi$ & $\stackrel{\Xi}{\Xi}$ & $\stackrel{\leftrightarrow}{g}$ & $\mid \begin{array}{l}\vec{b} \\
\dot{0} \\
i\end{array}$ & $\begin{array}{l}\text { 举 } \\
\text { 品 }\end{array}$ & 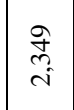 & ळ్ & 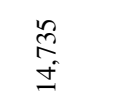 \\
\hline ț & \begin{tabular}{|l}
1 \\
\\
0 \\
0
\end{tabular} & 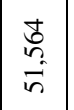 & 灾 & సे & 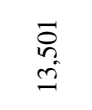 & $\underset{-}{\stackrel{t}{G}}$ & $\begin{array}{l}n \\
\tilde{n} \\
\tilde{n} \\
\tilde{n}\end{array}$ & $\begin{array}{l}\text { : } \\
\text { के }\end{array}$ & $\underset{i}{\text { 导 }}$ & in & 草 \\
\hline క్రి & 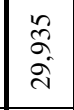 & \begin{tabular}{|l}
$\vec{\infty}$ \\
$\dot{o}$ \\
$\dot{q}$
\end{tabular} & $\begin{array}{l}\infty \\
\substack{0 \\
\infty \\
\infty}\end{array}$ & లె & 离 & $\stackrel{\infty}{\stackrel{\infty}{=}}$ & 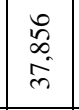 & 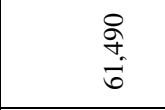 & $\underset{i}{\vec{j}}$ & 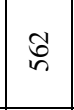 & $\begin{array}{l}\text { 足 } \\
\stackrel{\alpha}{=}\end{array}$ \\
\hline స్త్ & \begin{tabular}{|l|}
$\hat{0}$ \\
0 \\
0
\end{tabular} & 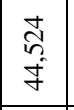 & $\underset{\infty}{\stackrel{0}{m}}$ & $\underset{\sim}{\infty}$ & $\begin{array}{l}\frac{\tilde{m}}{2} \\
\underline{n}\end{array}$ & : & 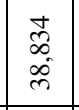 & 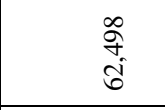 & 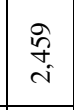 & in & 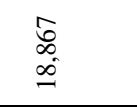 \\
\hline$\overline{\mathrm{J}}$ & 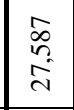 & $\begin{array}{c}\tilde{b} \\
\text { on } \\
\infty \\
\infty\end{array}$ & 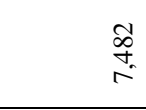 & ì & 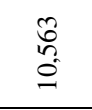 & $\underset{g}{-}$ & $\underset{f}{ \pm}$ & 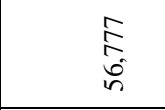 & $\frac{\vec{\infty}}{\vec{i}}$ & 导 & 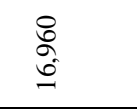 \\
\hline हั & \begin{tabular}{|l|}
$\infty$ \\
0 \\
0 \\
0 \\
\\
\end{tabular} & 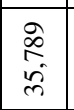 & : & సె & $\begin{array}{c}\approx \\
\substack{1 \\
\infty \\
\infty}\end{array}$ & 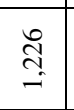 & 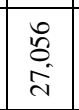 & 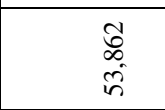 & $\stackrel{5}{5}$ & $\stackrel{\infty}{\curvearrowright}$ & 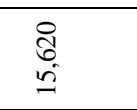 \\
\hline ga & \begin{tabular}{|l|l}
$n$ \\
$\infty$ \\
0 \\
di \\
\end{tabular} & 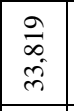 & : & $\underset{d}{\stackrel{9}{d}}$ & $\begin{array}{l}\text { 㟥 } \\
\text { s. }\end{array}$ & 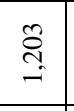 & 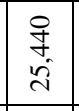 & $\begin{array}{l}\circ \\
\frac{8}{8} \\
0\end{array}$ & $\begin{array}{l}\text { ô } \\
\text { i }\end{array}$ & $\mid \begin{array}{l}\infty \\
⿱ 亠 乂\end{array}$ & 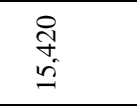 \\
\hline$\stackrel{\infty}{\stackrel{\infty}{\partial}}$ & 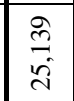 & $\begin{array}{l}\text { 盖 } \\
\text { d }\end{array}$ & 总 & $\stackrel{\infty}{\sim}$ & $\begin{array}{l}\stackrel{8}{\infty} \\
\infty\end{array}$ & 孚 & \begin{tabular}{|l|}
\multicolumn{1}{|c}{} \\
$\tilde{\pi}$ \\
$\tilde{y}$
\end{tabular} & $\begin{array}{l}\text { th } \\
\text { ơ } \\
\text { o. }\end{array}$ & $\begin{array}{l}\text { o. } \\
\text { i. }\end{array}$ & $\underset{\substack{\infty \\
\infty}}{ }$ & 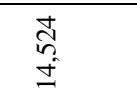 \\
\hline$\stackrel{5}{g}$ & \begin{tabular}{|l|} 
\\
0 \\
0 \\
0 \\
\end{tabular} & 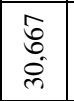 & 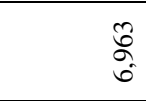 & $\stackrel{0}{d}$ & $\underset{\widetilde{2}}{\stackrel{2}{*}}$ & 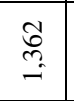 & 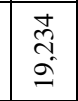 & 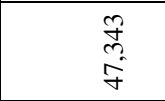 & $\begin{array}{l}\infty \\
\stackrel{\infty}{\circ} \\
\stackrel{-}{-}\end{array}$ & $\bar{g}$ & $\begin{array}{l}\text { 㐫 } \\
\stackrel{0}{2}\end{array}$ \\
\hline$\stackrel{\circ}{\circ}$ & \begin{tabular}{|l|}
$\overrightarrow{0}$ \\
$\mathrm{j}$ \\
$\mathrm{i}$
\end{tabular} & $\begin{array}{l}0 \\
\infty \\
\infty \\
\vdots \\
\vdots\end{array}$ & స్త్ర & ते & $\begin{array}{l}0 \\
0 \\
0.0 \\
0.0\end{array}$ & 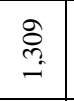 & 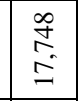 & 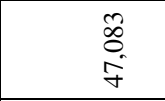 & 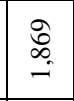 & \& & $\begin{array}{l}\frac{a}{f} \\
\text { If }\end{array}$ \\
\hline$\stackrel{0}{\circ}$ & \begin{tabular}{|l|} 
\\
0 \\
$\infty$ \\
0 \\
0
\end{tabular} & \begin{tabular}{|l}
$\vec{b}$ \\
\\
\end{tabular} & 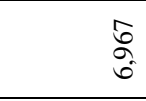 & ते & $\begin{array}{l}\infty \\
\infty \\
b^{2} \\
n^{2}\end{array}$ & 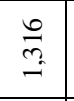 & \begin{tabular}{|l}
$\infty$ \\
$\stackrel{0}{0}$ \\
$\underline{0}$
\end{tabular} & $\begin{array}{l}\overrightarrow{5} \\
\dot{b} \\
\vec{f}\end{array}$ & : & $\underset{ల}{\infty}$ & $\begin{array}{l}\infty \\
\text { o } \\
\text { d }\end{array}$ \\
\hline I & 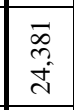 & 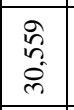 & $\begin{array}{l}\frac{\infty}{\infty} \\
0 \\
0\end{array}$ & 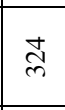 & $\begin{array}{l}\text { هo } \\
\stackrel{8}{i n}\end{array}$ & 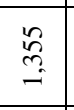 & 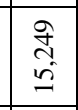 & 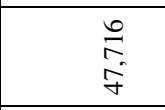 & 芯 & ర్ల & $\begin{array}{l}\bar{F} \\
\mathrm{~F}\end{array}$ \\
\hline$\stackrel{0}{g}$ & \begin{tabular}{|l|} 
\\
0 \\
0 \\
0 \\
0
\end{tabular} & 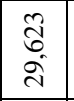 & 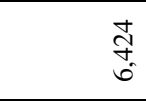 & $\underset{\infty}{\infty}$ & is & $\stackrel{\substack{0 \\
\hdashline}}{-}$ & $\begin{array}{l}\hat{m} \\
\hat{g}\end{array}$ & $\begin{array}{l}\text { वi } \\
\text { ơ }\end{array}$ & $\stackrel{\infty}{\stackrel{\infty}{c}}$ & $D_{i}$ & 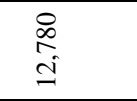 \\
\hline ईี & 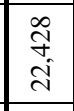 & 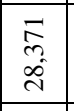 & $\begin{array}{l}\bar{o} \\
\vec{b}_{0}^{\circ}\end{array}$ & $\underset{\sim}{\infty}$ & $\underset{f}{\stackrel{0}{f}}$ & \begin{tabular}{|l}
$\vec{m}$ \\
$\stackrel{m}{-}$
\end{tabular} & \begin{tabular}{l}
\multirow{2}{*}{} \\
İ \\
In
\end{tabular} & $\begin{array}{l}\bar{\infty} \\
p_{2} \\
\sigma \\
\sigma \\
\end{array}$ & 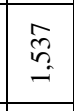 & ळे & $\begin{array}{l}\vec{c}_{\infty} \\
\stackrel{=}{=}\end{array}$ \\
\hline $\bar{g}$ & \begin{tabular}{|l|} 
\\
ôे \\
$\dot{\vec{j}}$ \\
\end{tabular} & 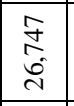 & 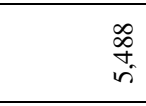 & is & $\stackrel{\infty}{\underset{f}{f}}$ & $\overrightarrow{\vec{g}_{-}}$ & 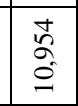 & 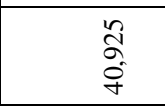 & 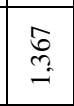 & $\stackrel{\circ}{m}$ & 总 \\
\hline పे & \begin{tabular}{|l|} 
\\
8 \\
0 \\
$i$
\end{tabular} & 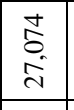 & 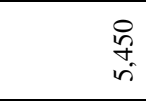 & $\infty_{i}^{\infty}$ & 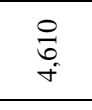 & $\stackrel{2}{2}$ & $\begin{array}{l}\text { 总 } \\
\text { o. }\end{array}$ & $\begin{array}{l}03 \\
0 \\
0 \\
0 \\
0\end{array}$ & 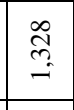 & 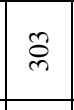 & 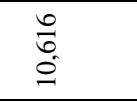 \\
\hline ڤ & $\begin{array}{l}0 \\
\delta \\
\delta\end{array}$ & 尺े & 岕 & $\begin{array}{l}0 \\
8 \\
8\end{array}$ & 苞 & 兽 & 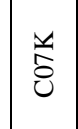 & $\stackrel{\circ}{8}$ & $\begin{array}{l}\mathscr{0} \\
\ddot{8} \\
\tilde{u}\end{array}$ & $\begin{array}{l}\text { O } \\
\ddot{8}\end{array}$ & 害 \\
\hline 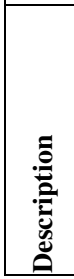 & 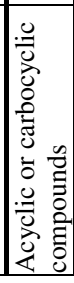 & & 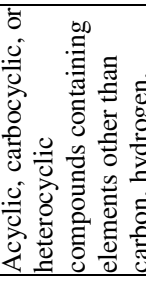 & & 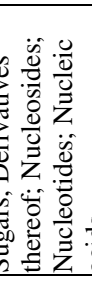 & 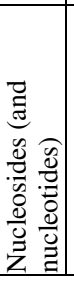 & 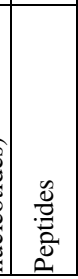 & 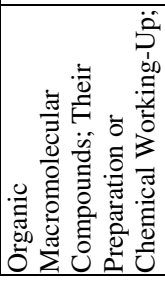 & 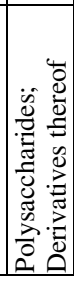 & & 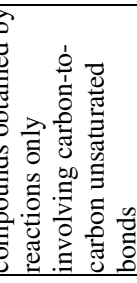 \\
\hline
\end{tabular}




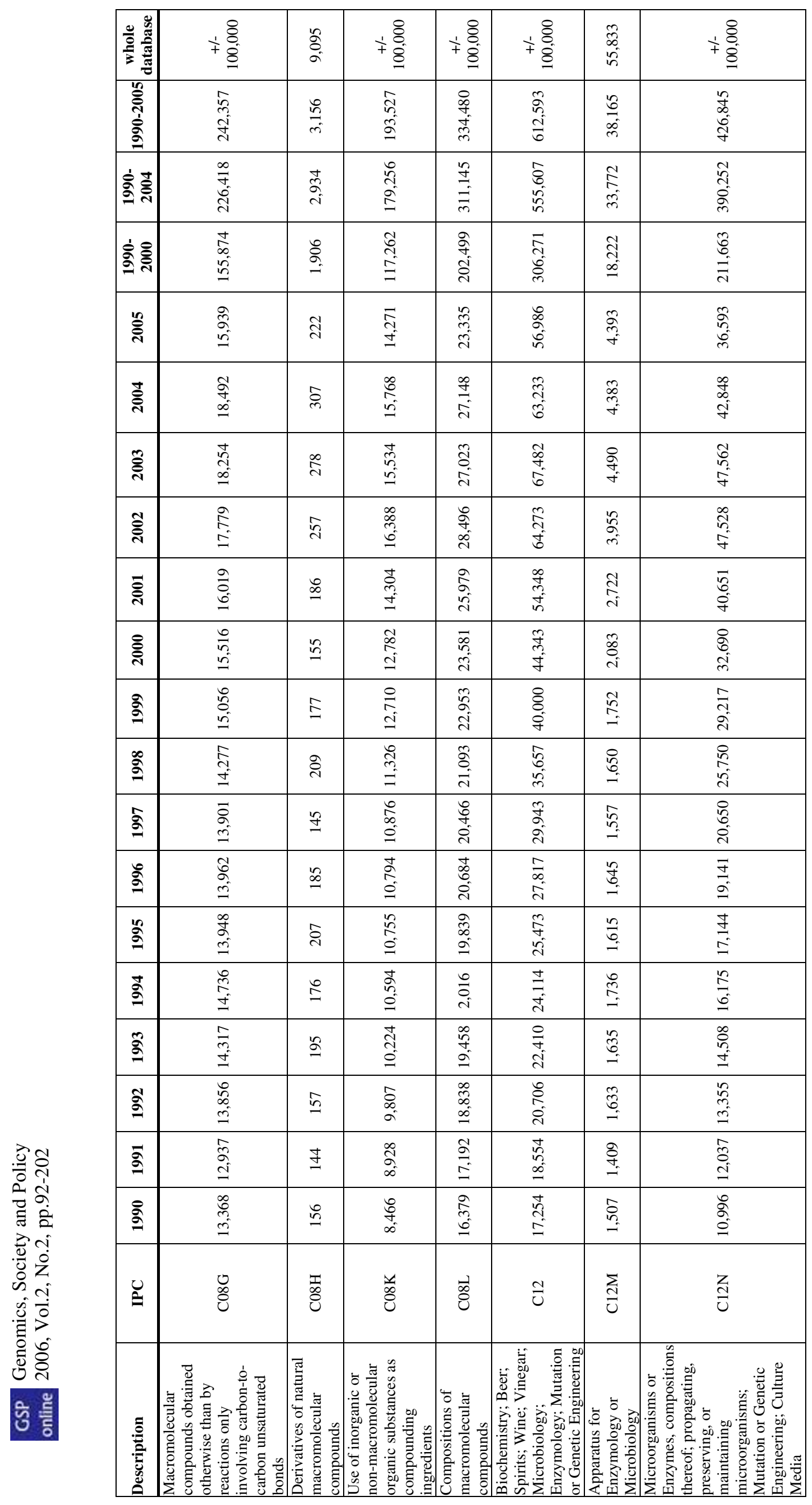




\begin{tabular}{|c|c|c|c|c|c|c|c|c|}
\hline 商 & $+\frac{8}{+}+\frac{0}{8}$ & $\begin{array}{l}8 \\
+ \\
+\end{array}$ & $\underset{\substack{+\infty}}{\stackrel{\infty}{*}}$ & $\begin{array}{l}m \\
0 \\
\dot{j} \\
\dot{m}\end{array}$ & 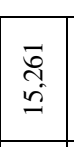 & $\begin{array}{l}8 \\
+ \\
+ \\
+ \\
0\end{array}$ & & 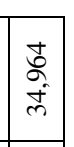 \\
\hline 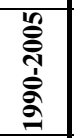 & $\begin{array}{l}\infty \\
\alpha^{\circ} \\
\sigma^{\prime}\end{array}$ & 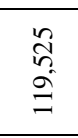 & ¿̊ & $\begin{array}{c}\stackrel{\wp}{0} \\
\stackrel{m}{m} \\
\end{array}$ & 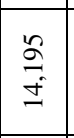 & $\begin{array}{l}\bar{y} \\
\text { ầ } \\
\end{array}$ & $\stackrel{\infty}{+}$ & 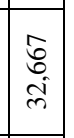 \\
\hline 言 & $\begin{array}{l}0 \\
\frac{0}{2} \\
\frac{2}{2}\end{array}$ & $\begin{array}{l} \pm \\
\text { à } \\
\text { gे } \\
\end{array}$ & $\begin{array}{l}\text { 守 } \\
\text { S }\end{array}$ & \begin{tabular}{|l|}
$\vec{E}$ \\
$\infty$ \\
id \\
\end{tabular} & \begin{tabular}{|l}
\multirow{\pi}{4}{} \\
İ \\
\end{tabular} & 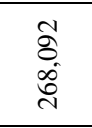 & $\mathcal{f}$ & 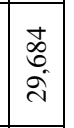 \\
\hline 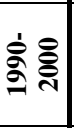 & 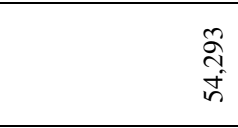 & $\begin{array}{l}\infty \\
\infty \\
\infty \\
\infty\end{array}$ & $\frac{8}{m}$ & $\frac{a}{m_{2}}$ & \begin{tabular}{|l|}
\multirow{2}{*}{} \\
$\stackrel{\sigma}{\sigma}$ \\
$\dot{\sigma}$
\end{tabular} & 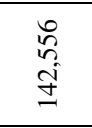 & $\begin{array}{l}\text { Fे } \\
\text { فी }\end{array}$ & 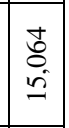 \\
\hline 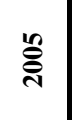 & $\begin{array}{l}\text { Iै } \\
\text { in }\end{array}$ & 商 & $\stackrel{\infty}{\stackrel{\infty}{n}}$ & $\stackrel{t}{2}$ & 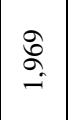 & \begin{tabular}{l} 
号 \\
\multirow{2}{*}{}
\end{tabular} & $b^{n}$ & $\begin{array}{l}\infty \\
\infty \\
a \\
i \\
\end{array}$ \\
\hline ఫั & 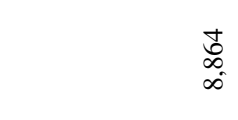 & $\begin{array}{l}0 \\
0 \\
\text { i } \\
\text { I }\end{array}$ & $\widetilde{\infty}$ & $\stackrel{s}{g}$ & $\stackrel{n}{=}$ & 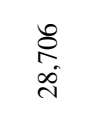 & $\begin{array}{l}\infty \\
\infty \\
0 \\
0 \\
0\end{array}$ & \begin{tabular}{|l|}
$\vec{m}$ \\
$\infty$ \\
$m$
\end{tabular} \\
\hline క్ & $\begin{array}{l}\stackrel{0}{0} \\
\stackrel{0}{0}\end{array}$ & $\begin{array}{l}\stackrel{Q}{+} \\
\stackrel{+}{=}\end{array}$ & $\tilde{s}$ & $\begin{array}{l}\underset{f}{1} \\
\infty\end{array}$ & 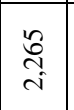 & $\begin{array}{l}\infty \\
\infty \\
\infty \\
\infty\end{array}$ & : & 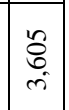 \\
\hline క్ & $\begin{array}{l}\text { 吕 } \\
\stackrel{0}{0}\end{array}$ & 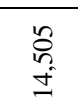 & 5 & $\begin{array}{c}\mathfrak{g} \\
\dot{f}\end{array}$ & ह̂. & 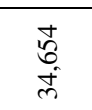 & 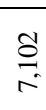 & $\underset{\substack{\bar{\infty} \\
c}}{\bar{c}}$ \\
\hline$\overline{\mathrm{c}}$ & $\begin{array}{l}\stackrel{a}{0} \\
\infty\end{array}$ & 㟢 & $\overline{\text { in }}$ & $\begin{array}{l}\infty \\
\stackrel{\circ}{i} \\
\dot{i}\end{array}$ & $\underset{\Im}{\stackrel{్}{-}}$ & बิ & 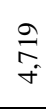 & $\mid \begin{array}{c}0 \\
0 \\
0 \\
-1\end{array}$ \\
\hline ક્ปે & 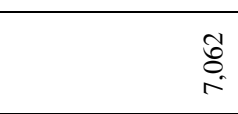 & 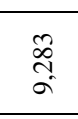 & $\vec{n}$ & 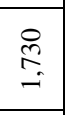 & $\bar{\infty}_{\infty}$ & 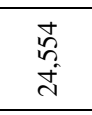 & fy & 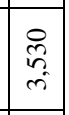 \\
\hline बे & $\overbrace{\substack{\infty \\
0 \\
0}}$ & $\begin{array}{l}\text { ơ } \\
\text { do } \\
\infty\end{array}$ & iิ & $\stackrel{9}{7}$ & $\stackrel{n}{2}$ & $\begin{array}{l}\infty \\
\stackrel{\infty}{0} \\
\stackrel{-}{+} \\
\end{array}$ & $\begin{array}{l}\text { D. } \\
\text { in }\end{array}$ & \begin{tabular}{|c|}
8 \\
$i$ \\
$i$ \\
\end{tabular} \\
\hline$\stackrel{\infty}{2}$ & 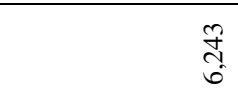 & $\begin{array}{l}\widehat{n} \\
\hat{c}\end{array}$ & ț & 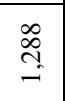 & 吉 & 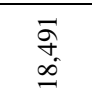 & 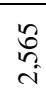 & $\begin{array}{l}\overrightarrow{5} \\
\overrightarrow{2}\end{array}$ \\
\hline$\hat{g}$ & $\begin{array}{l}\frac{n}{2} \\
\omega^{2}\end{array}$ & 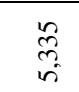 & $\stackrel{q}{d}$ & $\overrightarrow{0}$ & $\stackrel{\infty}{\infty} \stackrel{\infty}{\circ}$ & 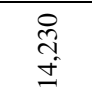 & $\begin{array}{l}\stackrel{0}{0} \\
\text { i. }\end{array}$ & $\begin{array}{c} \\
6 \\
0 \\
-1\end{array}$ \\
\hline$\stackrel{\circ}{\circ}$ & 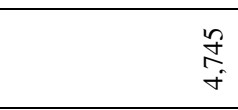 & 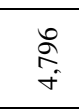 & $\tilde{\widetilde{\tau}}$ & హิ & 㤐 & $\begin{array}{l}\text { I } \\
\text { I }\end{array}$ & $\underset{g}{g}$ & $\frac{9}{\mathrm{I}}$ \\
\hline$\stackrel{0}{\mathrm{~g}}$ & $\begin{array}{l}\frac{\infty}{n} \\
\frac{n}{f}\end{array}$ & 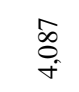 & $\stackrel{ \pm}{\sim}$ & 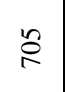 & $\stackrel{f}{f}$ & $\begin{array}{l}\stackrel{0}{a} \\
\stackrel{-}{=}\end{array}$ & 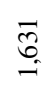 & $\underset{-}{\overrightarrow{8}}$ \\
\hline 奈 & 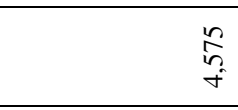 & స్ & $\stackrel{\infty}{\simeq}$ & $\begin{array}{l}\infty \\
\infty \\
i\end{array}$ & $\stackrel{n}{n}$ & 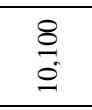 & $\stackrel{\infty}{0}$ & $\stackrel{2}{\infty}$ \\
\hline$\stackrel{0}{\sigma}$ & 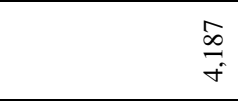 & 哭 & $\stackrel{\infty}{\circ}$ & $\stackrel{\circ}{\&}$ & $\overline{\mathrm{a}}$ & $\underset{\infty}{\frac{7}{7}}$ & in & iे \\
\hline 亏ั & ڤેे & $\begin{array}{l}\text { के } \\
\text { iे }\end{array}$ & $\stackrel{\infty}{=}$ & \& & $\ddot{i}$ & $\begin{array}{l}\infty \\
\stackrel{\infty}{\kappa}\end{array}$ & F & t \\
\hline $\bar{\Xi}$ & $\begin{array}{l}\infty \\
\omega^{\infty} \\
\omega^{2}\end{array}$ & 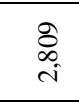 & $\stackrel{\text { cे }}{\circ}$ & $\stackrel{n}{m}$ & తి & $\begin{array}{l}0 \\
\text { ă } \\
0\end{array}$ & $\bar{q}$ & $\underset{\sim}{\pi}$ \\
\hline$\stackrel{\text { పू }}{ }$ & I্ & 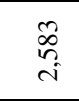 & $\stackrel{\circ}{\circ}$ & fै & $\stackrel{\infty}{\cong}$ & $\frac{8}{0}$ & ra & $\vec{m}$ \\
\hline$\stackrel{\varrho}{\varrho}$ & z & 窇 & $\begin{array}{l}\text { 离 } \\
\text { 窵 } \\
\text { Un }\end{array}$ & 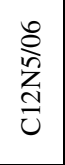 & $\begin{array}{l}\infty \\
\text { 离 } \\
\text { d } \\
\text { un }\end{array}$ & 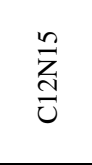 & & 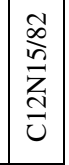 \\
\hline 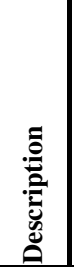 & 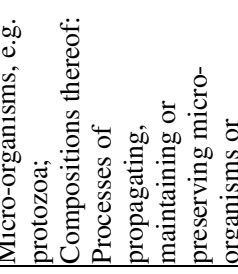 & 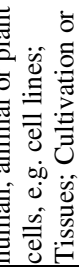 & 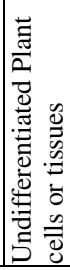 & & 槖 & 题 & & 常 \\
\hline
\end{tabular}




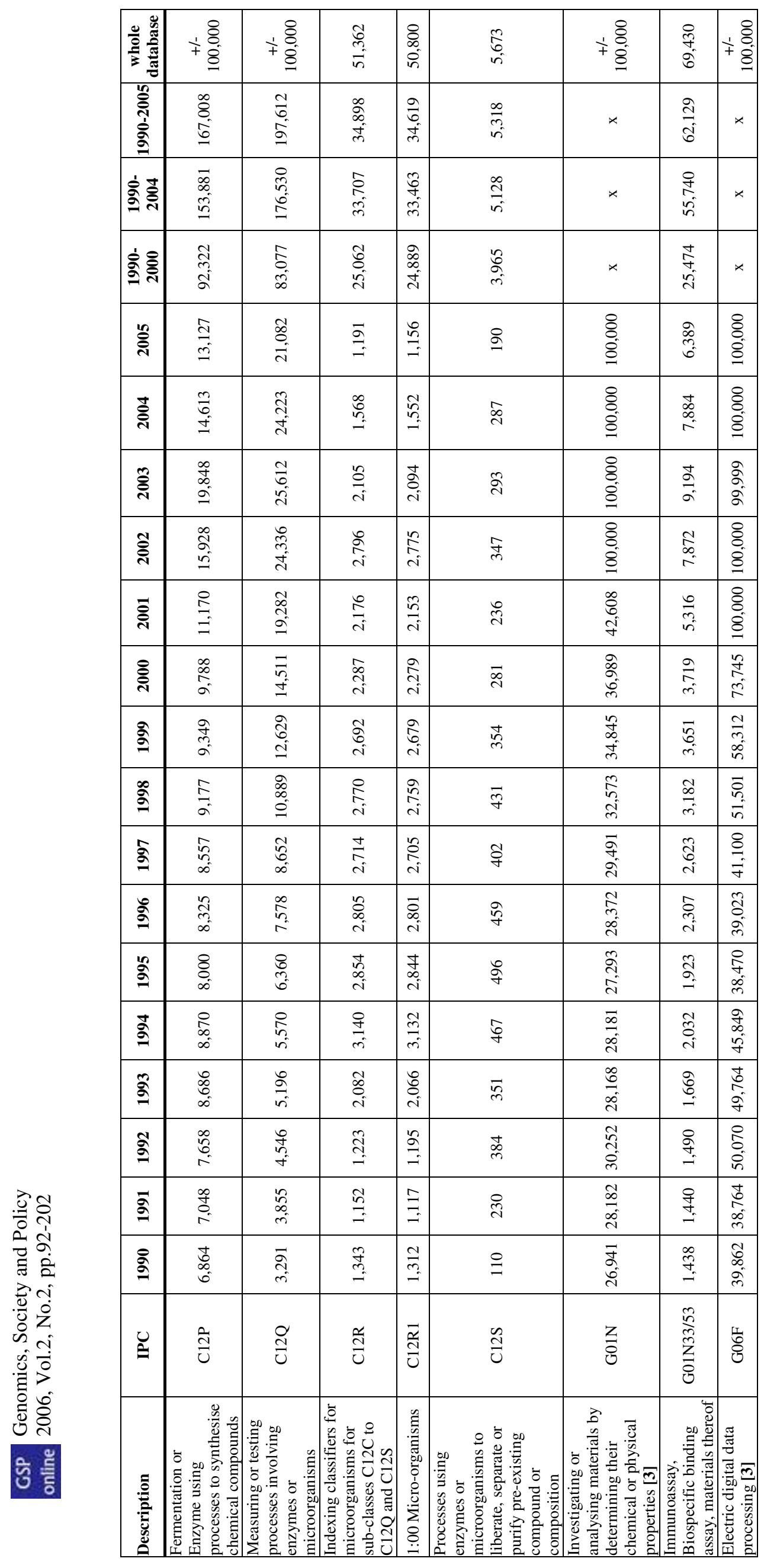




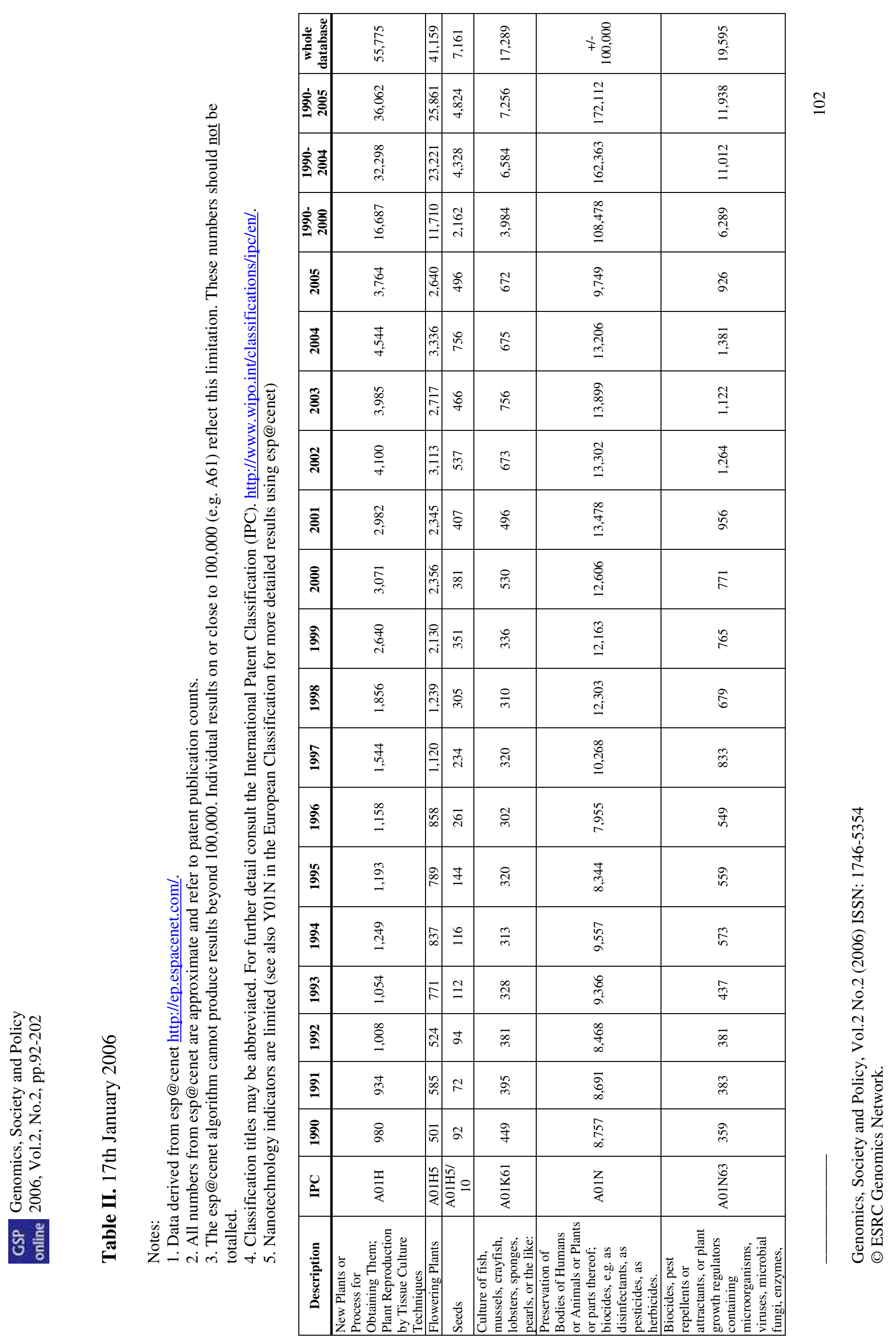




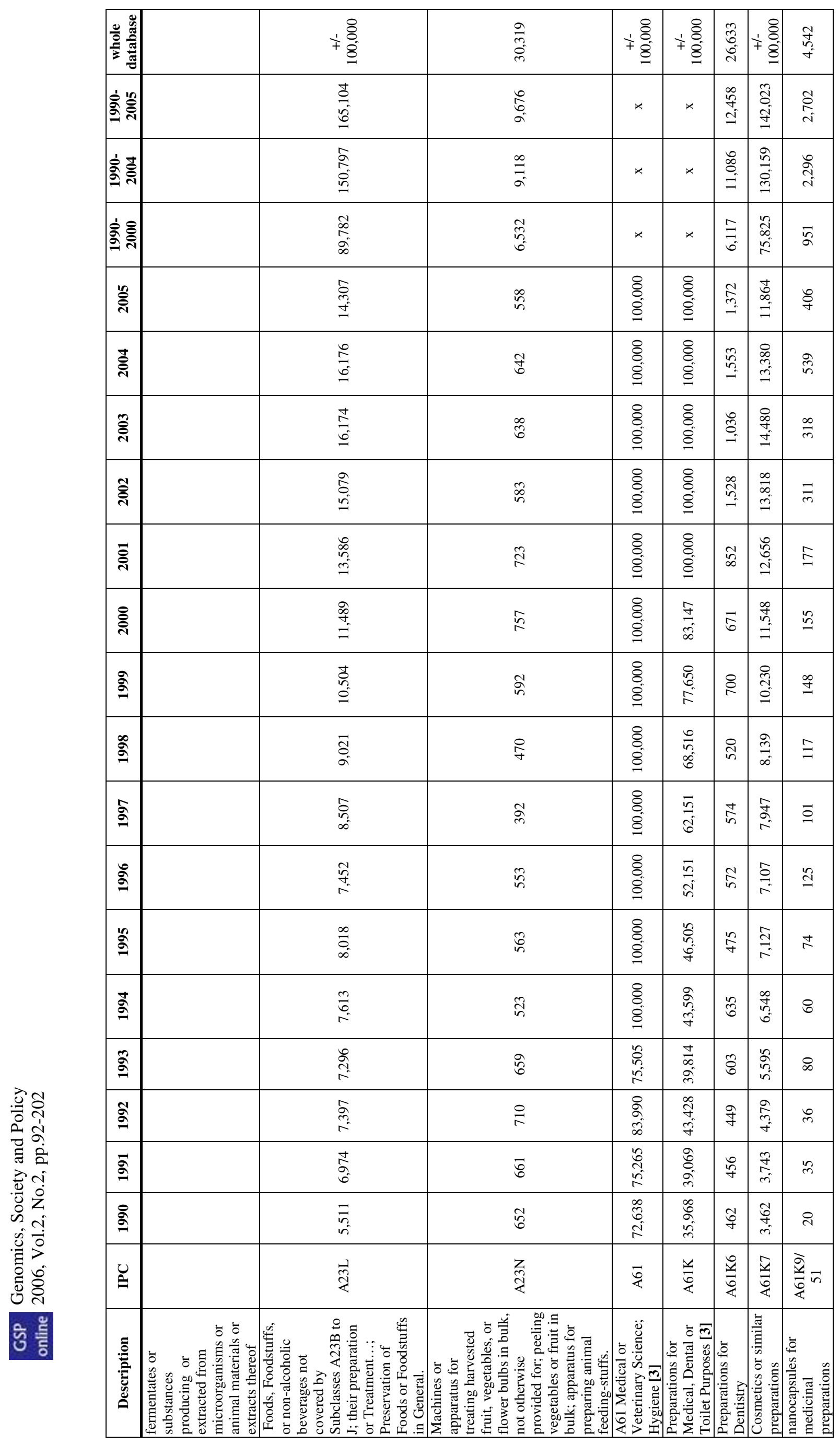




\begin{tabular}{|c|c|c|c|c|c|c|c|c|c|c|c|}
\hline 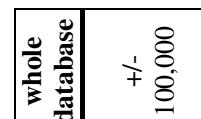 & $+\stackrel{8}{+}$ & लू & 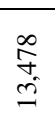 & $\begin{array}{l}\text { त्र } \\
\text { त्र }\end{array}$ & 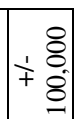 & 范 & $+\frac{8}{+0}$ & 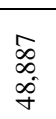 & $\begin{array}{l}\text { के } \\
\text { के }\end{array}$ & $\begin{array}{l}\infty \\
\infty \\
\infty \\
0\end{array}$ & $+\frac{8}{+}$ \\
\hline $\mid$\begin{tabular}{l|l}
0 \\
0
\end{tabular} & 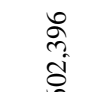 & $\begin{array}{l}7 \\
f \\
0\end{array}$ & $\begin{array}{c}\text { âd } \\
\text { a. }\end{array}$ & $\begin{array}{l}\tilde{\delta} \\
\dot{ \pm}\end{array}$ & $\begin{array}{l}\text { 今े } \\
\text { के } \\
\text { ơ }\end{array}$ & $\frac{n}{0}$ & $\begin{array}{l}\overrightarrow{0} \\
0 \\
0 \\
0 \\
0\end{array}$ & $\begin{array}{l}\infty \\
\infty \\
\infty \\
\infty\end{array}$ & $\begin{array}{l}\text { ț } \\
\text { ș }\end{array}$ & $\begin{array}{l}\infty \\
\infty \\
\infty \\
0\end{array}$ & $\begin{array}{l}5 \\
8 \\
8\end{array}$ \\
\hline
\end{tabular}

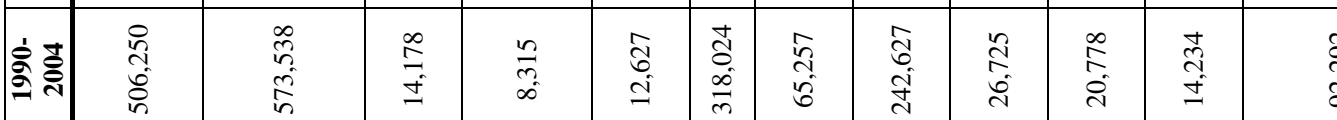

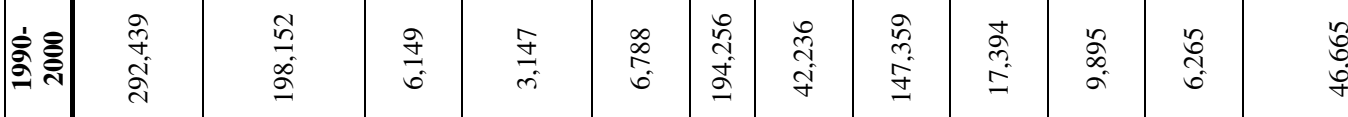

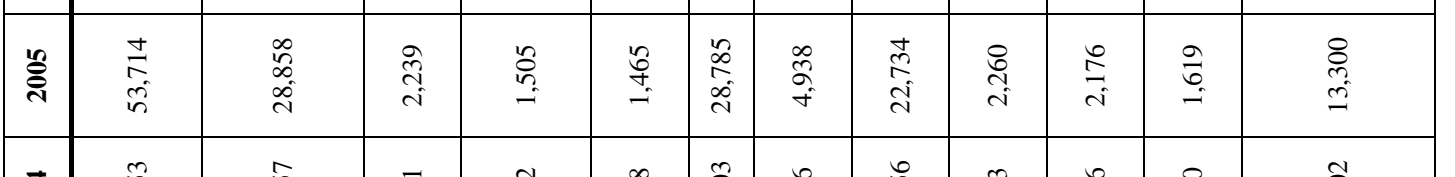

\begin{tabular}{|c|c|c|c|c|c|c|c|c|c|c|c|c|}
\hline క్స్త & $\begin{array}{l}n \\
\delta \\
0 \\
5\end{array}$ & $\begin{array}{l}\tilde{b} \\
\infty \\
\infty \\
\infty\end{array}$ & $\begin{array}{l}\overrightarrow{5} \\
\text { d }\end{array}$ & & 罗 & $\begin{array}{c}\text { Oे } \\
\text { De }\end{array}$ & $\stackrel{0}{\underline{n}}$ & $\begin{array}{l}0 \\
\stackrel{0}{0} \\
0\end{array}$ & 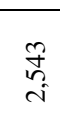 & 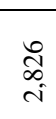 & 胥 & $\frac{\widetilde{\delta}}{\mathrm{I}}$ \\
\hline & 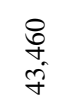 & $\begin{array}{l}\frac{n}{0} \\
i \\
i\end{array}$ & 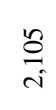 & İ & $\bar{g}$ & $\begin{array}{l}\overline{7} \\
\text { di }\end{array}$ & $\begin{array}{l}\text { o } \\
\text { th } \\
\text { in }\end{array}$ & $\begin{array}{l}\text { ô. } \\
\text { in }\end{array}$ & $\underset{\substack{+i}}{ }$ & సे & $\begin{array}{l}\text { D. } \\
\text { i }\end{array}$ & $\begin{array}{l}\stackrel{t}{0} \\
\text { I }\end{array}$ \\
\hline & 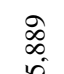 & $?$ & $\stackrel{ \pm}{\partial}$ & ఏ్ల్ & $\overline{\mathrm{g}}$ & $\stackrel{\infty}{v}$ & : & $\stackrel{0}{0}$ & ন্ป & $g$ & $\vec{m}$ & ర్ర \\
\hline
\end{tabular}

\begin{tabular}{|c|c|c|c|c|c|c|c|c|c|c|c|c|}
\hline క్ష & $\begin{array}{l}\infty \\
0 \\
\text { o. } \\
\text { n }\end{array}$ & $\stackrel{\frac{v}{c}}{m}$ & $\frac{\mathrm{d}}{\mathrm{N}}$ & $\stackrel{5}{0}$ & 总 & 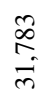 & $\begin{array}{l}0 \\
\text { in } \\
\text { n }\end{array}$ & 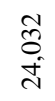 & 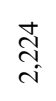 & s. & $\overrightarrow{\overrightarrow{\vec{d}}}$ & $\begin{array}{l}\vec{\circ} \\
\Rightarrow\end{array}$ \\
\hline స్త్ & $\begin{array}{l}\text { ठृ } \\
\text { סृ }\end{array}$ & $\begin{array}{l}\text { o } \\
\text { D. }\end{array}$ & 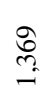 & $\stackrel{6}{2}$ & 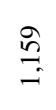 & $\begin{array}{l}\text { d. } \\
\text { ते }\end{array}$ & $\begin{array}{l}\text { gr } \\
\text { in }\end{array}$ & $\begin{array}{l}\vec{a} \\
\vec{i}\end{array}$ & $\frac{d}{i}$ & $\begin{array}{l}\text { d. } \\
\text { i } \\
i\end{array}$ & $\begin{array}{l}0 \\
0 \\
-1\end{array}$ & $\begin{array}{l}0 \\
0 \\
\infty \\
0\end{array}$ \\
\hline ఫ్సి & 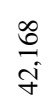 & $\begin{array}{l}+ \\
0 \\
o \\
\text { d }\end{array}$ & $\vec{\infty}$ & 壳 & $\stackrel{8}{\circ}$ & $\begin{array}{l}\hat{~} \\
\text { సે }\end{array}$ & $\begin{array}{l}\vec{m} \\
\text { min }\end{array}$ & $\frac{\mathbb{d}}{\stackrel{0}{\infty}}$ & 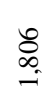 & $\stackrel{\text { g }}{q}$ & s. & 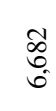 \\
\hline$a$ & $\frac{0}{n !}$ & శి & $F$ & वे & ஜ & $\begin{array}{l}\infty \\
\infty\end{array}$ & $\stackrel{\infty}{\infty}$ & ñ & $\vec{E}$ & تే & $\approx$ & f \\
\hline
\end{tabular}

\begin{tabular}{|c|c|c|c|c|c|c|c|c|c|c|c|c|}
\hline gे & $\begin{array}{l}0 \\
n \\
n \\
n\end{array}$ & $\begin{array}{l}\text { ते } \\
\text { ते }\end{array}$ & F & भे & $\stackrel{2}{\curvearrowright}$ & 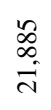 & 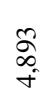 & $\begin{array}{l}\tilde{n} \\
\underline{n} \\
\underline{0}\end{array}$ & $\underset{=}{\mathbb{E}}$ & 范 & న్ & 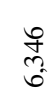 \\
\hline$\stackrel{20}{2}$ & $\begin{array}{l}\bar{\alpha} \\
\text { oे }\end{array}$ & 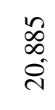 & 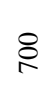 & 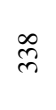 & $\stackrel{\circ}{\circ}$ & 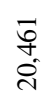 & 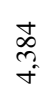 & 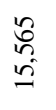 & $\underset{⿱ n}{\vec{n}}$ & 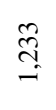 & ळం & $\begin{array}{l}\tilde{n} \\
\hat{b}\end{array}$ \\
\hline$\stackrel{\hat{g}}{ }$ & $\begin{array}{l}\hat{a} \\
\dot{\alpha}\end{array}$ & $\begin{array}{l}\stackrel{m}{d} \\
\Omega \\
\text { I }\end{array}$ & $\stackrel{0}{\infty}$ & $\ddot{q}$ & م⿱ & 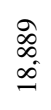 & $\begin{array}{l}\text { 守 } \\
f\end{array}$ & 占 & $\begin{array}{l}\infty \\
\substack{\infty \\
i}\end{array}$ & 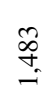 & $\stackrel{\overrightarrow{0}}{-}$ & 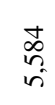 \\
\hline ه & $=$ & के & & 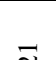 & $\cong$ & $\infty$ & $\approx$ & $m$ & $\stackrel{+}{+}$ & $=$ & & $\varnothing$ \\
\hline
\end{tabular}

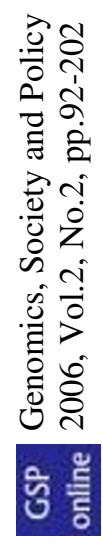

\begin{tabular}{|c|c|c|c|c|c|c|c|c|c|c|c|c|}
\hline & & & & & & & & & & & & \\
\hline gू & 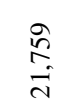 & $\begin{array}{l}\tilde{n} \\
\hat{n} \\
\underline{0}\end{array}$ & సิ & 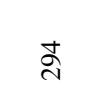 & જે & 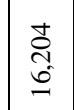 & $\underset{\sim}{\vec{f}}$ & 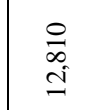 & ণิ ָे & $\stackrel{\circ}{\circ}$ & 总 & 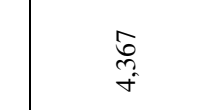 \\
\hline$\stackrel{8}{g}$ & 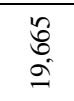 & $\begin{array}{l}\text { o } \\
0 \\
\text { s. }\end{array}$ & के & $\stackrel{\sim}{\circ}$ & $\tilde{n}$ & $\begin{array}{l}\hat{\sigma} \\
\dot{\jmath} \\
\dot{y}\end{array}$ & $\begin{array}{l}\tilde{b} \\
\dot{w} \\
m\end{array}$ & $\begin{array}{l}n \\
\mathbb{I} \\
\mathbb{I}\end{array}$ & 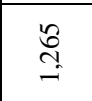 & $\vec{F}$ & $\vec{\lambda}$ & 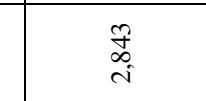 \\
\hline ธี & $\begin{array}{l}0+1 \\
\text { d } \\
\text { d. }\end{array}$ & $\begin{array}{l}\text { m } \\
\hat{j} \\
\dot{J}\end{array}$ & \&े & $\bar{\Xi}$ & 芯 & $\begin{array}{l}\overrightarrow{\mathrm{S}} \\
\dot{ \pm} \\
\end{array}$ & $\begin{array}{l}\hat{\sigma} \\
\text { is } \\
\end{array}$ & $\stackrel{\varrho}{\stackrel{2}{=}}$ & 寿 & बे & 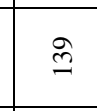 & 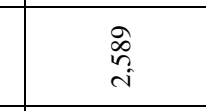 \\
\hline ఏ్ & $\begin{array}{l}\text { ma } \\
\hat{n} \\
\text { ते }\end{array}$ & $\begin{array}{l}\stackrel{\circ}{10} \\
\stackrel{n}{n}\end{array}$ & 처 & $\stackrel{\Xi}{\Xi}$ & $\tilde{\sigma}$ & $\begin{array}{l}\vec{\pi} \\
\stackrel{\text { In }}{\Omega}\end{array}$ & 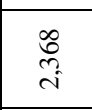 & 㕝 & $\underset{\mathrm{S}}{\vec{S}}$ & $\stackrel{\circ}{m}$ & $\therefore$ & $\vec{g}$ \\
\hline$\stackrel{8}{2}$ & $\begin{array}{l}\text { ते } \\
\text { d. }\end{array}$ & $\begin{array}{l}\hat{\tilde{b}} \\
\dot{J}\end{array}$ & $\frac{m}{m}$ & fo & $\stackrel{m}{m}$ & 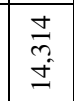 & 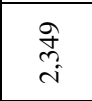 & $\begin{array}{l}0 \\
\text { in } \\
\text { o. }\end{array}$ & $\stackrel{n}{=}$ & $\underset{\sim}{~}$ & in & $S_{-}$ \\
\hline 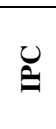 & 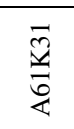 & 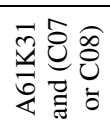 & & & & 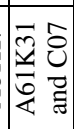 & 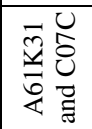 & & & & $\overrightarrow{\underline{z}}$ & $\begin{array}{l}n \\
\hat{n} \\
0 \\
0\end{array}$ \\
\hline 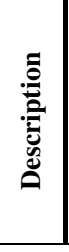 & 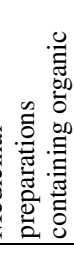 & 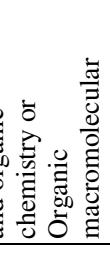 & 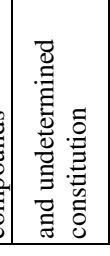 & 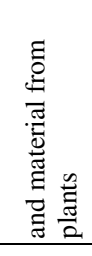 & 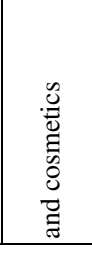 & & 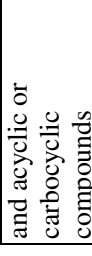 & 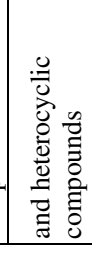 & 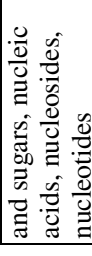 & 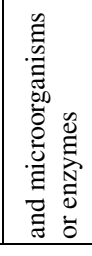 & 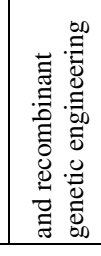 & 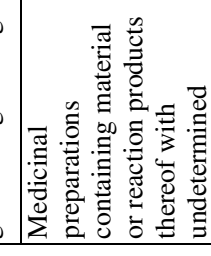 \\
\hline
\end{tabular}




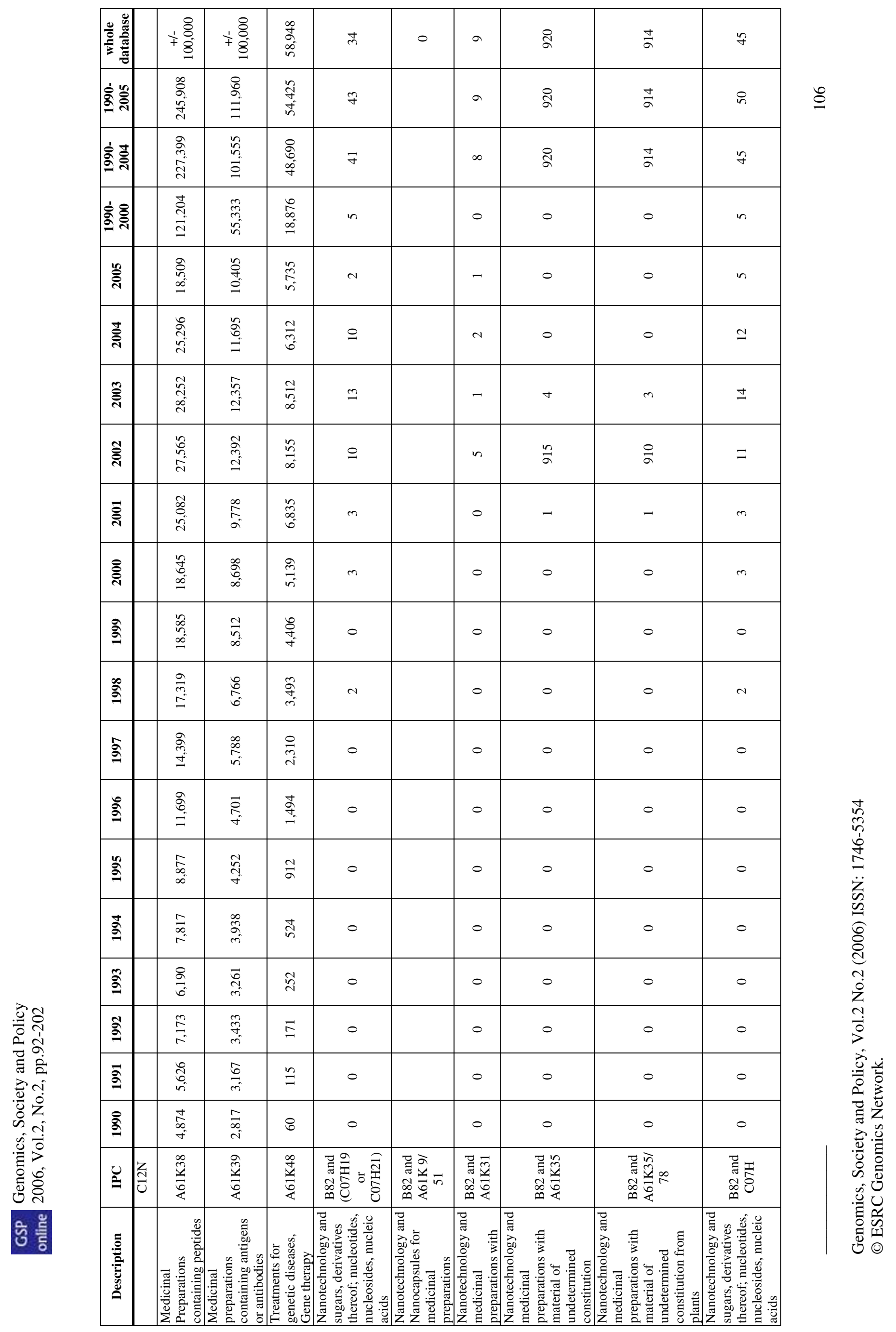




\begin{tabular}{|c|c|c|c|c|c|c|c|c|c|c|c|c|c|}
\hline 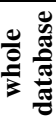 & $\stackrel{8}{\circ}$ & $\stackrel{+}{n}$ & 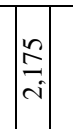 & 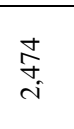 & $\mid$\begin{tabular}{ll}
0 \\
+ \\
+ \\
+ \\
\\
\hdashline
\end{tabular} & $\begin{array}{l}1 \\
+\frac{8}{0} \\
+\stackrel{8}{-}\end{array}$ & $\left|\begin{array}{rl} & 0 \\
+ & 0 \\
+ & 0 \\
\hdashline & 0\end{array}\right|$ & $\begin{array}{l}\frac{8}{8} \\
+\frac{8}{8} \\
\stackrel{8}{8}\end{array}$ & $\begin{array}{l}\text { ते } \\
\text { के }\end{array}$ & $\begin{array}{l}\dot{8} \\
+ \\
+ \\
\\
0\end{array}$ & 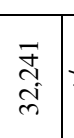 & 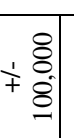 & 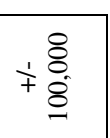 \\
\hline 亏亏亏 & in & $F$ & $=$ & $\underset{i}{\vec{J}}$ & * & 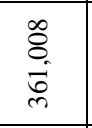 & 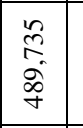 & $\begin{array}{l}0 \\
\text { ợ } \\
\text { g. }\end{array}$ & ले & 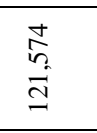 & 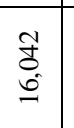 & 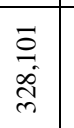 & 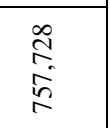 \\
\hline 言 & $\cong$ & F & : & 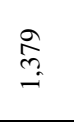 & $\star$ & 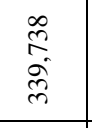 & 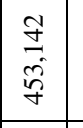 & के & $\underset{\vec{m}}{\vec{m}}$ & $\begin{array}{l}\stackrel{\circ}{0} \\
\stackrel{\Xi}{\Xi}\end{array}$ & $\begin{array}{l}\text { वे } \\
\text { in } \\
\end{array}$ & $\begin{array}{l}\text { o } \\
\text { 文 } \\
\text { d }\end{array}$ & 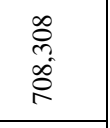 \\
\hline 客言 & 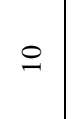 & $m$ & $F$ & తి & * & $\begin{array}{l}\text { ते } \\
\text { ते } \\
\text { ते }\end{array}$ & $\begin{array}{l}\text { aे } \\
\text { a. } \\
\text { d. }\end{array}$ & : & 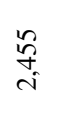 & $\begin{array}{l}0 \\
0 \\
0 \\
n \\
n\end{array}$ & \begin{tabular}{l}
$\infty$ \\
\multirow{2}{\infty}{} \\
$\sigma$
\end{tabular} & 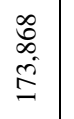 & 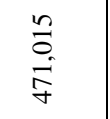 \\
\hline 今్ & $\infty$ & $\stackrel{+}{\sim}$ & $\frac{\infty}{f}$ & $\stackrel{\complement}{\curvearrowright}$ & 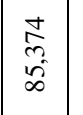 & 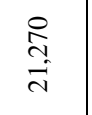 & 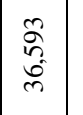 & $\begin{array}{l}\text { 总 } \\
\text { in }\end{array}$ & $\stackrel{\infty}{ \pm}$ & $\begin{array}{l}\infty \\
\vdots \\
\sigma \\
\sigma\end{array}$ & $\underset{\Xi}{-}$ & $\begin{array}{l}\text { fo } \\
\text { वृं }\end{array}$ & $\begin{array}{l}\text { वे } \\
\text { वे }\end{array}$ \\
\hline ఫ్ స్ & $\dot{m}$ & $=$ & $\frac{\lambda}{2}$ & $\underset{\infty}{\infty}$ & $\begin{array}{l}8 \\
8 \\
\dot{8} \\
\end{array}$ & 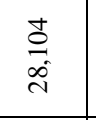 & $\begin{array}{l}\tilde{\hat{\sigma}} \\
\dot{\sigma} \\
\dot{\sigma}\end{array}$ & 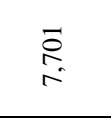 & 电 & $\begin{array}{l}\stackrel{\sim}{0} \\
\stackrel{\infty}{=}\end{array}$ & $\underset{\substack{g \\
=}}{\mathscr{g}}$ & $\begin{array}{c}\stackrel{+}{0} \\
\dot{m} \\
\end{array}$ & $\underset{\substack{n \\
\infty}}{=}$ \\
\hline ڤ్సે & $\tilde{m}$ & $\stackrel{\infty}{.}$ & 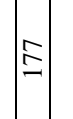 & 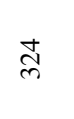 & 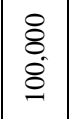 & 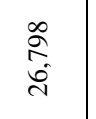 & 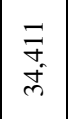 & $\begin{array}{l}\infty \\
\stackrel{\infty}{n} \\
\sim\end{array}$ & gे & 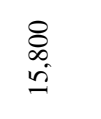 & वे & $\begin{array}{l}\vec{N} \\
0 \\
0 \\
\infty \\
\infty\end{array}$ & 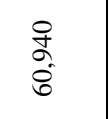 \\
\hline ธ్ & 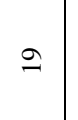 & 6 & $\stackrel{2}{a}$ & $\stackrel{\infty}{\underset{\sim}{2}}$ & 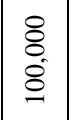 & $\begin{array}{l}\frac{\infty}{\stackrel{2}{2}} \\
\text { ते }\end{array}$ & 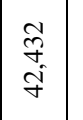 & $\stackrel{\overbrace{}}{\stackrel{\sigma}{r}}$ & 空 & 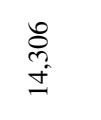 & $\stackrel{\Xi}{i}$ & $\begin{array}{l}\frac{6}{0} \\
\frac{n}{2} \\
m\end{array}$ & 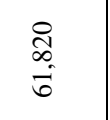 \\
\hline క్స్ & $\simeq$ & $m$ & 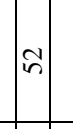 & $\stackrel{\varrho}{\varrho}$ & $\begin{array}{l}\stackrel{8}{0} \\
\stackrel{8}{0} \\
\end{array}$ & 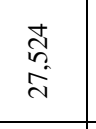 & 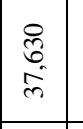 & 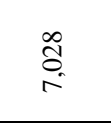 & $E$ & $\frac{q}{0}$ & ๙ু & 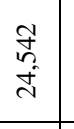 & 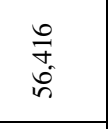 \\
\hline స్సి & 0 & $\mathrm{~A}$ & $\approx$ & f & 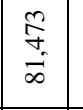 & $\begin{array}{l}\text { of } \\
\text { d } \\
\text { d }\end{array}$ & 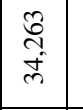 & 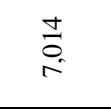 & $\bar{\Xi}$ & 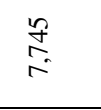 & $\vec{\infty}$ & 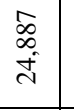 & $\begin{array}{l}\text { 寿 } \\
\vec{n}\end{array}$ \\
\hline बे & $m$ & - & $A$ & 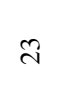 & 志 & $\begin{array}{l}\text { 売 } \\
\text { d }\end{array}$ & 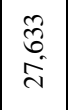 & 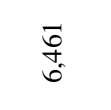 & I & 亭 & $\ddot{\mathscr{O}}$ & 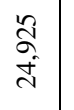 & $\begin{array}{l}\text { F } \\
\text { in } \\
\text { in }\end{array}$ \\
\hline$\stackrel{2}{g}$ & - & 0 & in & $\simeq$ & 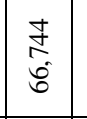 & $\underset{\vec{c}}{\vec{j}}$ & $\underset{\substack{0 \\
c}}{i}$ & 荵 & है & 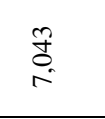 & in & $\begin{array}{l}\overrightarrow{x_{0}} \\
\vec{i}\end{array}$ & $\begin{array}{l}\vec{\sigma} \\
+ \\
+ \\
\dot{y}\end{array}$ \\
\hline Sิ & 0 & 0 & - & $a$ & $\begin{array}{l}\text { त्र } \\
\text { तु } \\
\end{array}$ & $\begin{array}{l}\text { ले } \\
\text { ले } \\
\text { ते }\end{array}$ & $\begin{array}{l}\text { त्र } \\
\text { हु } \\
\end{array}$ & $\begin{array}{l}\text { aे } \\
\text { in }\end{array}$ & $\stackrel{n}{n}$ & $\begin{array}{l}\infty \\
\substack{\infty \\
0 \\
0}\end{array}$ & $\stackrel{\text { g }}{\mathrm{g}}$ & $\begin{array}{l}0 \\
0 \\
0 \\
\text { s. } \\
\end{array}$ & 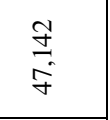 \\
\hline$\stackrel{\circ}{\circ}$ & 0 & 0 & - & $\checkmark$ & 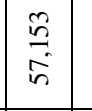 & $\begin{array}{l}\vec{\lambda} \\
\stackrel{\lambda}{I}\end{array}$ & $\begin{array}{l}\vec{E} \\
\text { D. } \\
\text { in } \\
\text {. }\end{array}$ & $\begin{array}{l}\vec{\sigma} \\
\text { in }\end{array}$ & $\stackrel{\infty}{\infty}$ & $\begin{array}{l}\vec{\infty} \\
\hat{n} \\
n^{2}\end{array}$ & 占 & $\begin{array}{l}\frac{\partial}{\partial} \\
\dot{I}\end{array}$ & $\begin{array}{l}\frac{a}{0} \\
\stackrel{0}{f}\end{array}$ \\
\hline ڤ̆ & 0 & 0 & - & 0 & $\begin{array}{l}n \\
\vdots \\
0 \\
0 \\
n \\
n\end{array}$ & 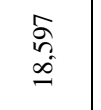 & 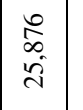 & $\begin{array}{l}\text { D. } \\
\text { hn } \\
\text { in }\end{array}$ & ণิ & $\begin{array}{l}\stackrel{m}{8} \\
i\end{array}$ & $\stackrel{\circ}{\stackrel{\circ}{-}}$ & $\begin{array}{l}0 \\
0 \\
\text { dे }\end{array}$ & 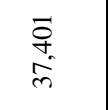 \\
\hline ڤ̆ & 0 & 0 & - & n & $\frac{\partial}{\vec{\gamma}}$ & $\begin{array}{l}\stackrel{8}{g} \\
\stackrel{g}{g}\end{array}$ & $\begin{array}{l}0 \\
0 \\
\text { in } \\
\end{array}$ & 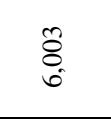 & $\overline{\mathrm{i}}$ & 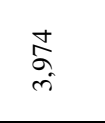 & $\underset{\infty}{+}$ & 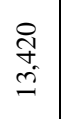 & 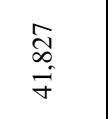 \\
\hline$\stackrel{8}{2}$ & 0 & 0 & $\sim$ & $r$ & $\begin{array}{l}0 \\
\text { : } \\
i \\
i \\
i n\end{array}$ & 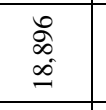 & 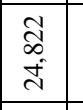 & $\frac{0}{i n}$ & $\overrightarrow{\vec{i}}$ & $\begin{array}{l}8 \\
\stackrel{8}{+} \\
\end{array}$ & $\overbrace{\infty}$ & $\begin{array}{l}\tilde{S} \\
\text { Oे } \\
\end{array}$ & $\begin{array}{l}9 \\
\text { Jे } \\
\text { in }\end{array}$ \\
\hline$\delta$ & 0 & 0 & 0 & 0 & 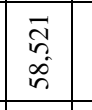 & 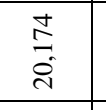 & 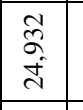 & $\begin{array}{l}\infty \\
\infty \\
\infty \\
i\end{array}$ & $\stackrel{\infty}{\stackrel{\infty}{\sim}}$ & $\begin{array}{l}\text { 足 } \\
\stackrel{n}{+}\end{array}$ & $\mid$ & 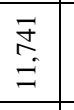 & $\begin{array}{l}\infty \\
\text { ते } \\
\infty \\
\infty\end{array}$ \\
\hline$\overline{\widehat{\sigma}}$ & 0 & 0 & 0 & 0 & 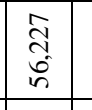 & $\begin{array}{l}\text { : } \\
\text { ते }\end{array}$ & 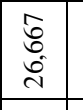 & $\begin{array}{l}\tilde{B} \\
\text { in }\end{array}$ & 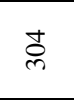 & 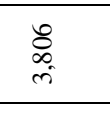 & $\stackrel{8}{2}$ & 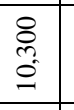 & $\begin{array}{l}\frac{\partial}{\vec{\partial}} \\
\frac{\partial}{\infty}\end{array}$ \\
\hline ప్ & 0 & 0 & - & in & 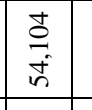 & $\begin{array}{l}\overline{\vec{\lambda}} \\
\stackrel{\Omega}{\Omega}\end{array}$ & $\begin{array}{l}\text { d. } \\
\text { di } \\
\end{array}$ & 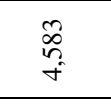 & $\stackrel{\Re}{m}$ & : & : & 童 & 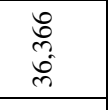 \\
\hline$\triangleq$ & 芽 & 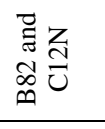 & 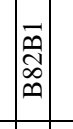 & $\begin{array}{l}\infty \\
\stackrel{0}{0} \\
\infty \\
\infty\end{array}$ & $\tilde{8}$ & 范 & 今ิ & 嶽 & : & 苞 & $\begin{array}{l}\stackrel{2}{\vec{T}} \\
\overrightarrow{0}\end{array}$ & 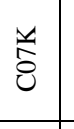 & $\stackrel{\infty}{8}$ \\
\hline$\stackrel{8}{\circ}$ & 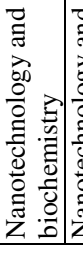 & 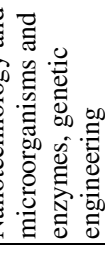 & & & & & & & & 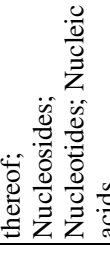 & & & 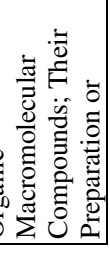 \\
\hline
\end{tabular}




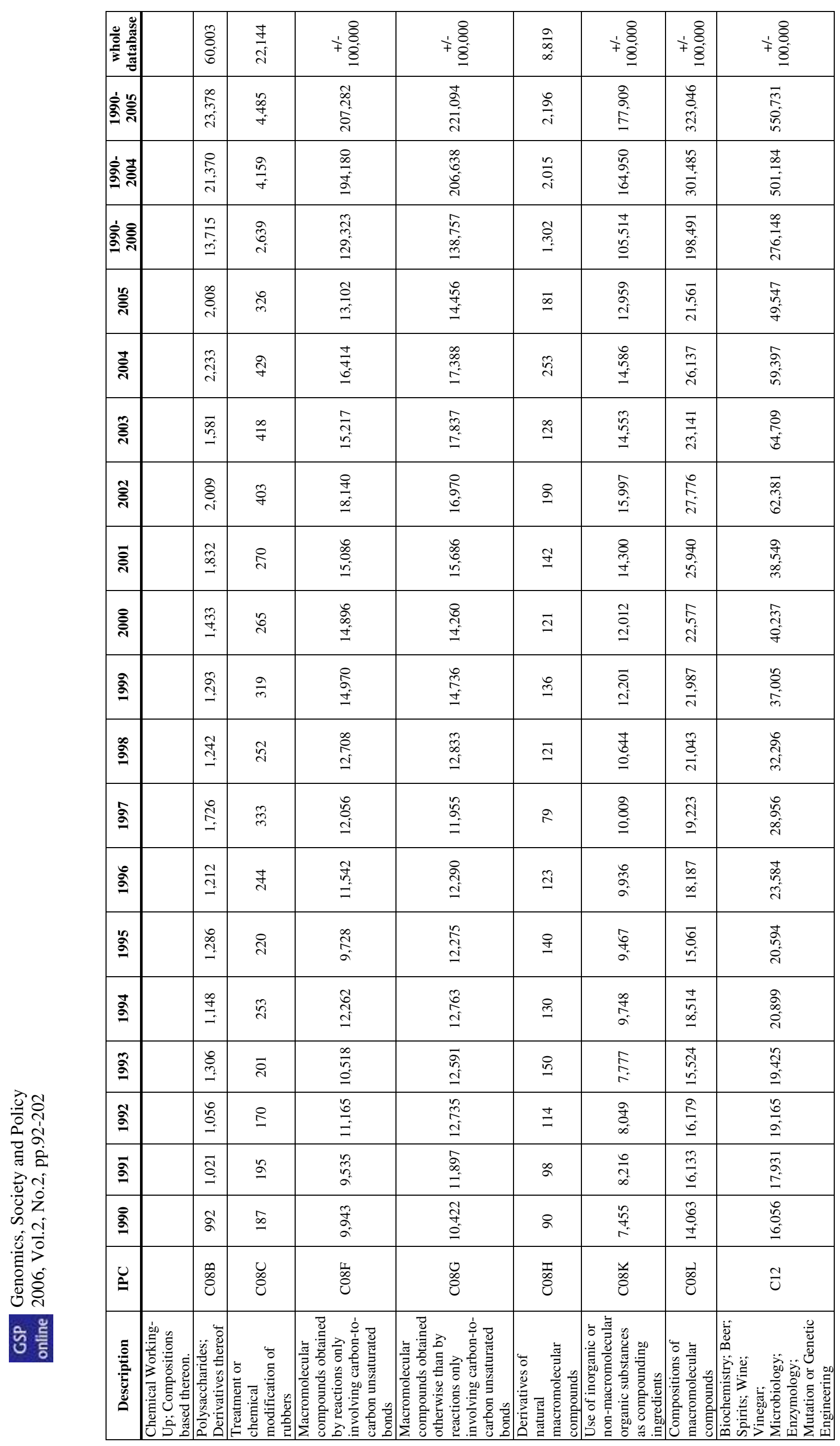




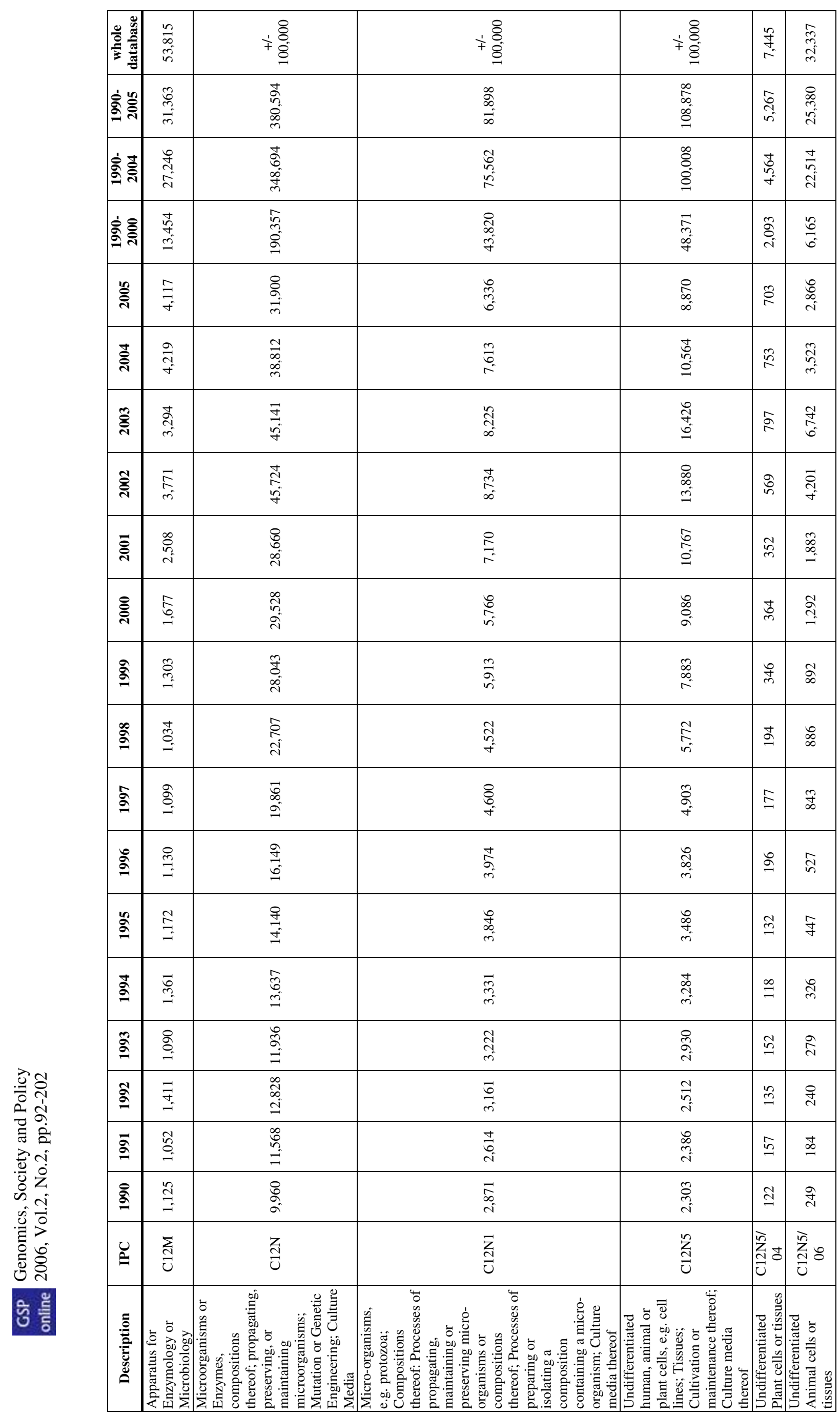




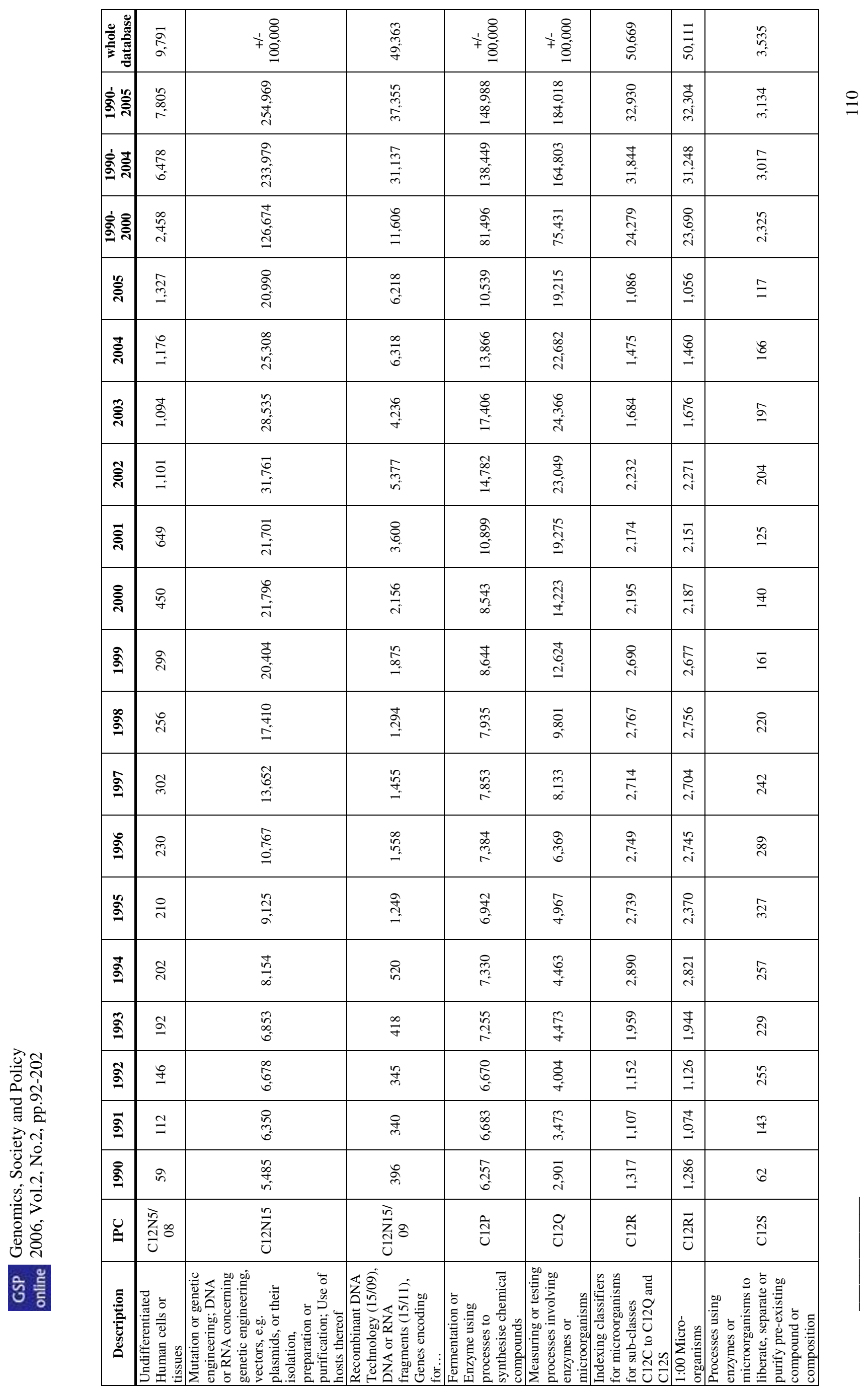




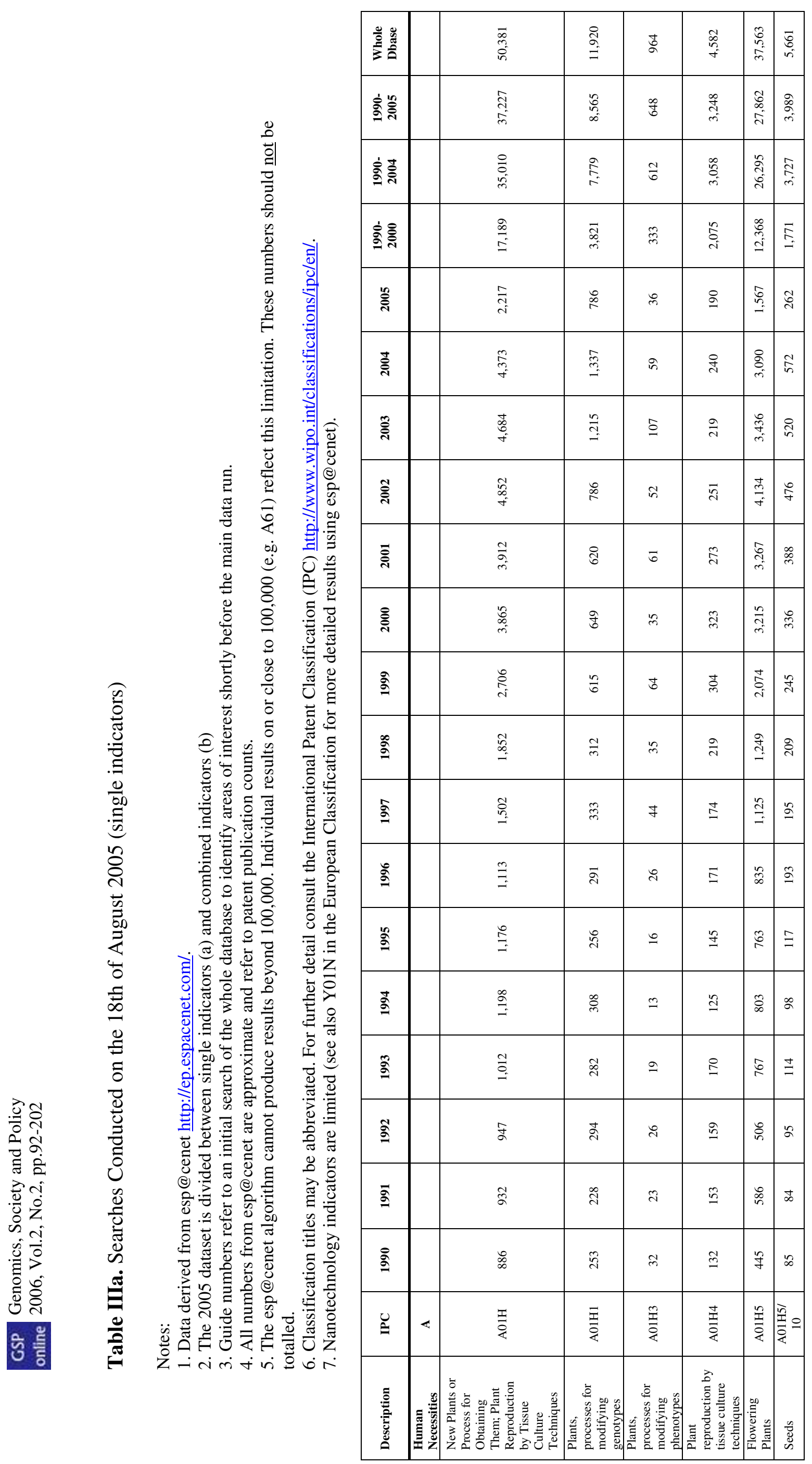




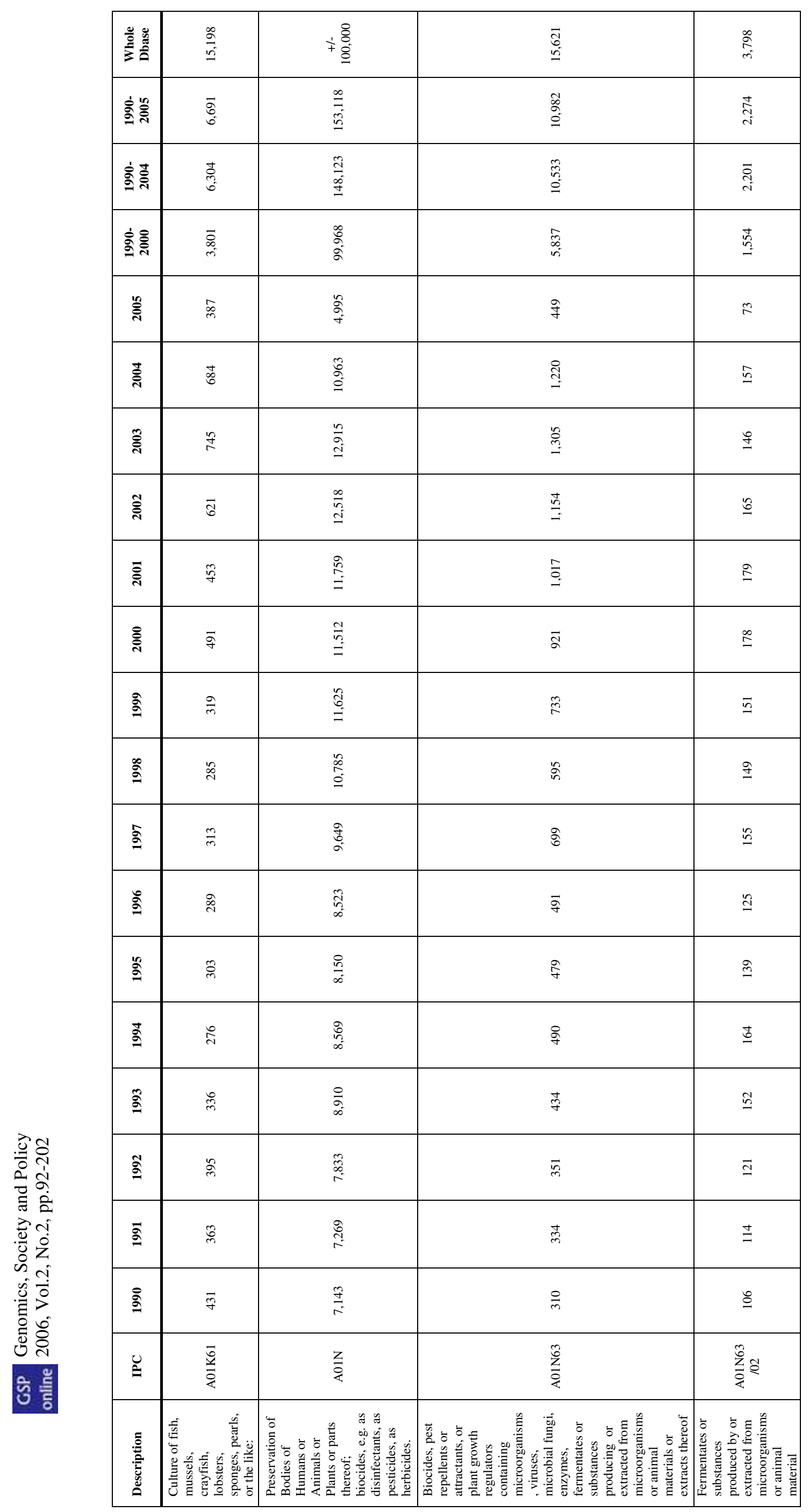




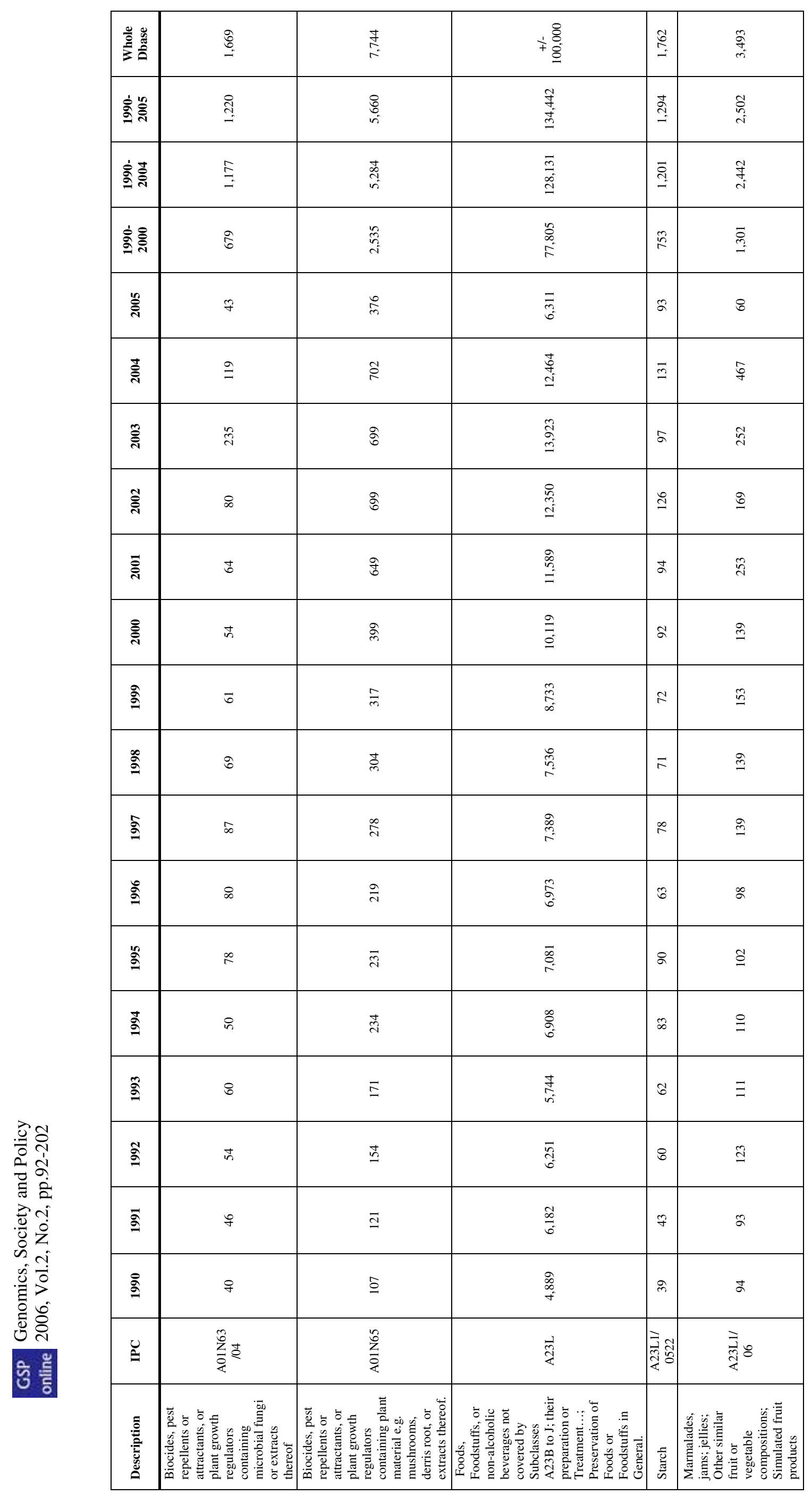




\begin{tabular}{|c|c|c|c|c|c|}
\hline 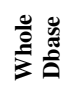 & $\begin{array}{l}\text { वे } \\
\end{array}$ & 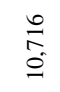 & f & 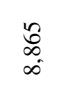 & 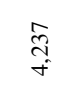 \\
\hline 言高 & $\stackrel{N}{=}$ & $\begin{array}{c}\text { d } \\
\text { in }\end{array}$ & $\hat{n}$ & $\begin{array}{l}\text { It } \\
\text { r. }\end{array}$ & 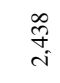 \\
\hline 紊言 & $\overline{\hat{g}_{0}}$ & శ్రే & $\stackrel{2}{i}$ & $\begin{array}{l}\frac{\infty}{5} \\
+ \\
+\end{array}$ & ते \\
\hline s. & 㞼 & 点 & $\stackrel{\circ}{7}$ & 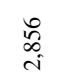 & 卓 \\
\hline 銥 & f & $\vec{d}$ & $\vec{d}$ & ฝั & s) \\
\hline 言 & $\stackrel{\infty}{0}$ & $\overrightarrow{\bar{\sigma}}$ & ra & $\vec{n}$ & สี \\
\hline 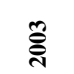 & 离 & 点 & $\Xi$ & 字 & 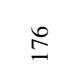 \\
\hline క్స్ & $\hat{\bar{b}}$ & 总 & $\sigma$ & 吉 & $\stackrel{ \pm}{g}$ \\
\hline ప్ & 架 & S & $\stackrel{\infty}{\llcorner}$ & iี & 王 \\
\hline స్ㅎํ & 吕 & $\bar{\sigma}$ & $\stackrel{+}{ \pm}$ & 品 & $\stackrel{\varrho}{\subseteq}$ \\
\hline ळे & $\stackrel{\infty}{\infty}$ & s. & q & $\nexists$ & $\ddot{n}$ \\
\hline$\stackrel{\infty}{\circ}$ & f & 志 & q & 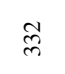 & $\Xi$ \\
\hline S & $F$ & 궘 & $\vec{a}$ & 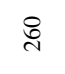 & 主 \\
\hline$\stackrel{\circ}{\circ}$ & i & 索 & $m$ & $\widetilde{\pi}$ & $\underline{n}$ \\
\hline$\stackrel{0}{g}$ & în & సี & $\stackrel{\infty}{\infty}$ & : & $\stackrel{\circ}{h}$ \\
\hline 奇 & g্ & $\tilde{a}$ & $\neq$ & 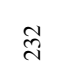 & $\stackrel{ \pm}{I}$ \\
\hline 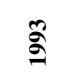 & $\stackrel{\infty}{\text { 呆 }}$ & 今े & $\infty$ & $\tilde{ส}$ & $\beth$ \\
\hline 亏ี & 索 & $\underset{\sim}{\mathbb{a}}$ & $\infty$ & 呆 & $\Xi$ \\
\hline $\bar{\sigma}$ & f & वू & $\underset{\sim}{\infty}$ & $\stackrel{\infty}{\infty}$ & $\cong$ \\
\hline دิ & $\Rightarrow$ & $\stackrel{d}{\circ}$ & $\stackrel{9}{ }$ & $\tilde{a}$ & 5 \\
\hline$\cong$ & $\overrightarrow{\bar{\lambda}}$ & जे & 궗류 & वृत्वृत & 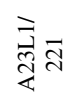 \\
\hline
\end{tabular}




\begin{tabular}{|c|c|c|c|c|c|c|c|c|c|c|}
\hline 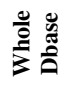 & 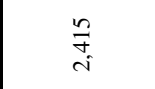 & $\begin{array}{l}\bar{B} \\
\text { in }\end{array}$ & $\begin{array}{l}\hat{\text { N }} \\
\text { re }\end{array}$ & مِ & $\underset{\sim}{\mathbb{t}}$ & $\underset{-}{8}$ & $\begin{array}{c}\tilde{O} \\
\vdots \\
\dot{\sigma} \\
\dot{w}\end{array}$ & $\begin{array}{l}\overrightarrow{\vec{o}} \\
\vec{i}\end{array}$ & $\begin{array}{l}\infty \\
\stackrel{2}{a} \\
\stackrel{+}{f}\end{array}$ & $\stackrel{\circ}{\neq}$ \\
\hline 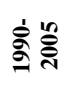 & $\stackrel{\circ}{\stackrel{2}{ت}}$ & $\begin{array}{l}\frac{t}{0} \\
i\end{array}$ & $\begin{array}{l}\infty \\
\stackrel{\infty}{-} \\
-1\end{array}$ & $\begin{array}{l}\vec{g} \\
\stackrel{+}{+}\end{array}$ & $\sqrt{\infty}$ & $\frac{n}{6}$ & $\begin{array}{l}\text { t } \\
\text { i } \\
\text { in }\end{array}$ & : & $\begin{array}{l}n \\
\mathfrak{r} \\
m\end{array}$ & 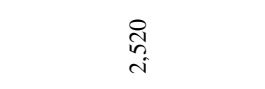 \\
\hline 今े & $\begin{array}{l}\infty \\
i n \\
i n\end{array}$ & $\underset{\substack{+\underset{c}{+}}}{ }$ & $\underset{-}{\hat{S}}$ & 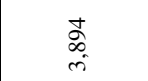 & $\frac{0}{\infty}$ & $\stackrel{\infty}{\infty}$ & 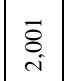 & $\stackrel{\infty}{\leftrightarrows}$ & $\begin{array}{l}\vec{F} \\
m \\
m\end{array}$ & 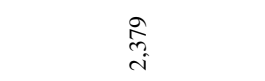 \\
\hline 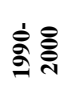 & $\stackrel{\infty}{\circ}$ & 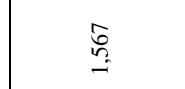 & $\stackrel{8}{\because}$ & 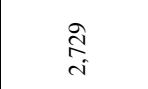 & ๙ิ & : & I & $\mathscr{8}$ & $\begin{array}{l}\infty \\
\stackrel{n}{c} \\
i\end{array}$ & 导 \\
\hline 甯 & $\cong$ & $\stackrel{-}{-}$ & $\vec{\infty}$ & $\bar{\Xi}$ & F & 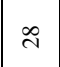 & $\approx$ & $\infty$ & $\stackrel{ \pm}{ \pm}$ & $\bar{\Xi}$ \\
\hline క్స & $\overline{\bar{\alpha}}$ & $\underset{\sim}{\circ}$ & $\stackrel{q}{\mathcal{F}}$ & $\stackrel{\widehat{A}}{\hat{N}}$ & $\approx$ & $\infty$ & 五 & 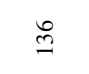 & $\underset{\sim}{\stackrel{t}{d}}$ & $\stackrel{\infty}{\sim}$ \\
\hline 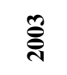 & $\hat{\imath}$ & $\frac{n}{N}$ & $\Xi$ & $\stackrel{m}{m}$ & $F$ & in & $\Xi$ & $\stackrel{\vartheta}{\exists}$ & 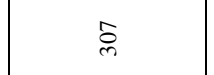 & 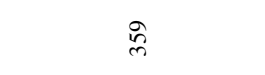 \\
\hline క్ڤ్ & छे & $\vec{d}$ & $\bar{n}$ & $\stackrel{\circ}{m}$ & $\stackrel{\infty}{n}$ & $\Varangle$ & $\stackrel{n}{n}$ & $\tilde{g}$ & ff & $\widetilde{\Omega}$ \\
\hline స్తి & $\underline{m}$ & 。ั & $\stackrel{\Xi}{\beth}$ & $\overrightarrow{\mathrm{d}}$ & 8 & g & $\cong$ & $\Xi$ & : & 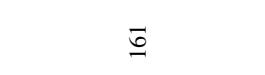 \\
\hline ఫ్సે & a & $\ddot{b}$ & के & $\vec{J}$ & in & वे & 导 & $\infty$ & శై & $\cong$ \\
\hline$\stackrel{\partial}{=}$ & $\stackrel{\infty}{\infty}$ & $\stackrel{\Xi}{\Xi}$ & $\stackrel{\infty}{=}$ & $\frac{n}{d}$ & F & 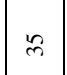 & $\cong$ & I & $\stackrel{\sim}{a}$ & $\stackrel{ \pm}{\stackrel{t}{2}}$ \\
\hline $\begin{array}{l}\infty \\
\stackrel{\infty}{\sigma}\end{array}$ & $\infty$ & 巳 & $\cong$ & $\underset{d}{\mathbb{N}}$ & in & f & తి & $\infty$ & 颃 & 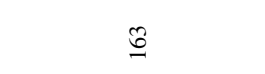 \\
\hline 产 & 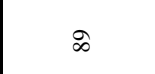 & $\stackrel{n}{n}$ & $\stackrel{t}{\stackrel{t}{a}}$ & ల్ల & 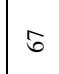 & $\approx$ & $\underset{I}{~}$ & 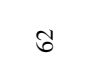 & స్సి & 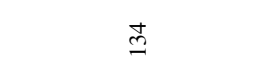 \\
\hline ڤ. & 8 & की & $\stackrel{\varrho}{\varrho}$ & तี & 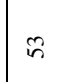 & ల & $\cong$ & $\infty$ & $\stackrel{\infty}{\sim}$ & $\stackrel{8}{\circ}$ \\
\hline$\stackrel{\circ}{\circ}$ & $\approx$ & $\underline{\square}$ & $\cong$ & 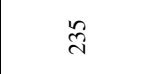 & if & $m$ & ปे & in & $\stackrel{d}{\mathbb{N}}$ & $\stackrel{\infty}{\circ}$ \\
\hline 索 & $\$$ & 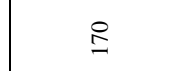 & $\stackrel{\Xi}{\Xi}$ & 웜 & $\approx$ & q & $\stackrel{ \pm}{I}$ & 5 & ¿্ণ & $\stackrel{\infty}{\stackrel{\circ}{2}}$ \\
\hline$\stackrel{0}{\sigma}$ & in & $\underline{n}$ & $\stackrel{\varrho}{\exists}$ & त্ন & $m$ & $\bar{\lambda}$ & $\stackrel{\circ}{\stackrel{\circ}{9}}$ & in & $\stackrel{t}{\mathrm{~g}}$ & $\stackrel{\infty}{=}$ \\
\hline$\stackrel{\Xi}{\sigma}$ & in & 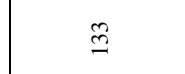 & 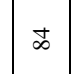 & ¿্ণ & $\bar{m}$ & 㝏 & 孚 & if & $\vec{m}$ & $\stackrel{8}{\circ}$ \\
\hline $\bar{\sigma}$ & $\hat{m}$ & $\infty$ & 8 & $\overline{\mathrm{d}}$ & $\stackrel{\infty}{q}$ & ì & g & $\tilde{m}$ & $\Phi$ & $\%$ \\
\hline$\stackrel{\circ}{g}$ & n & $\stackrel{\varrho}{\varrho}$ & $\infty$ & 乱 & q & 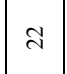 & さे & in & I & $\stackrel{2}{2}$ \\
\hline 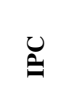 & $\overrightarrow{\vec{j}}$ & $\overrightarrow{\vec{\jmath}} \overline{\text { त्र }}$ & 局 & 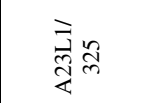 & 武 & न्तृ & 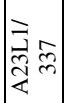 & 冚 & 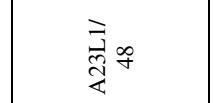 & 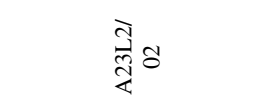 \\
\hline 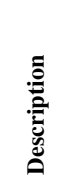 & 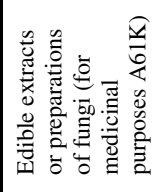 & 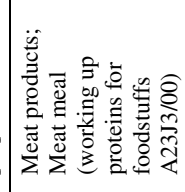 & 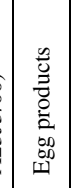 & 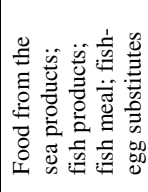 & 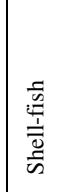 & 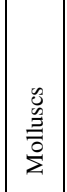 & 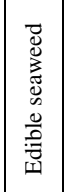 & 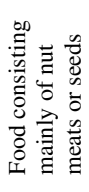 & 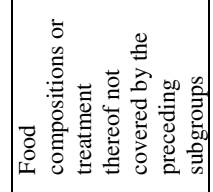 & 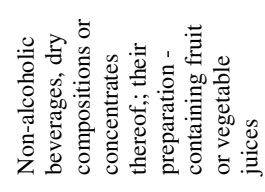 \\
\hline
\end{tabular}




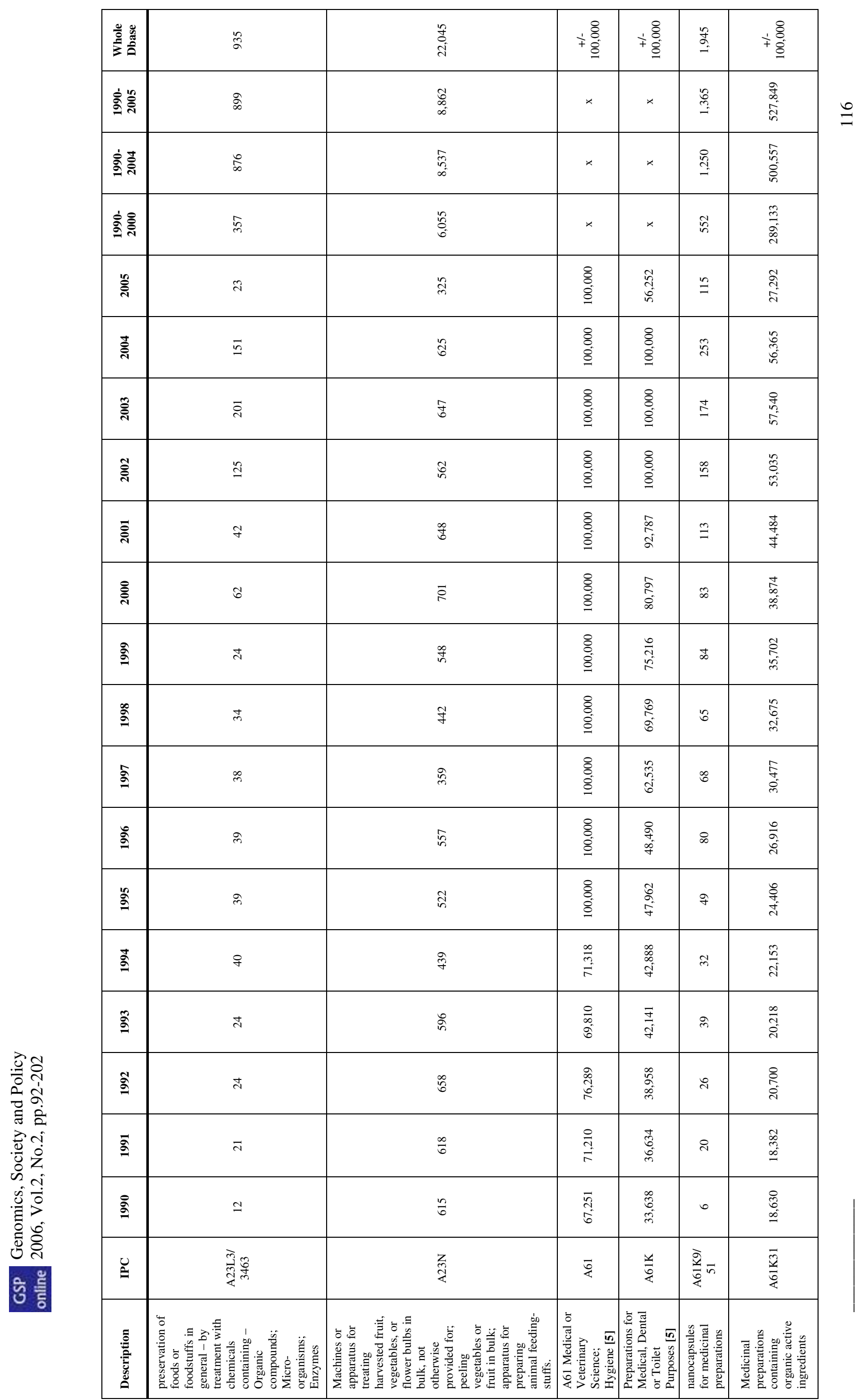




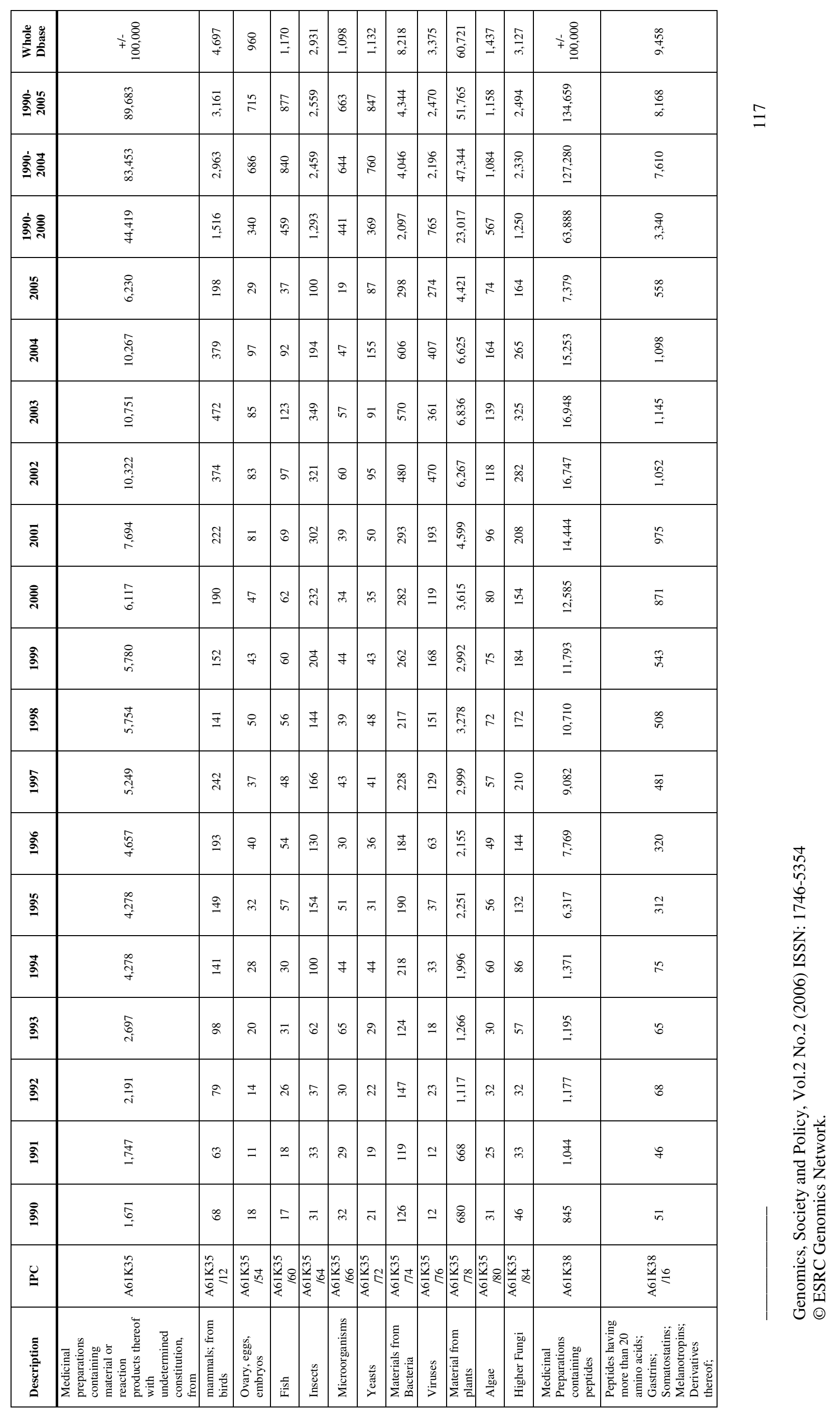




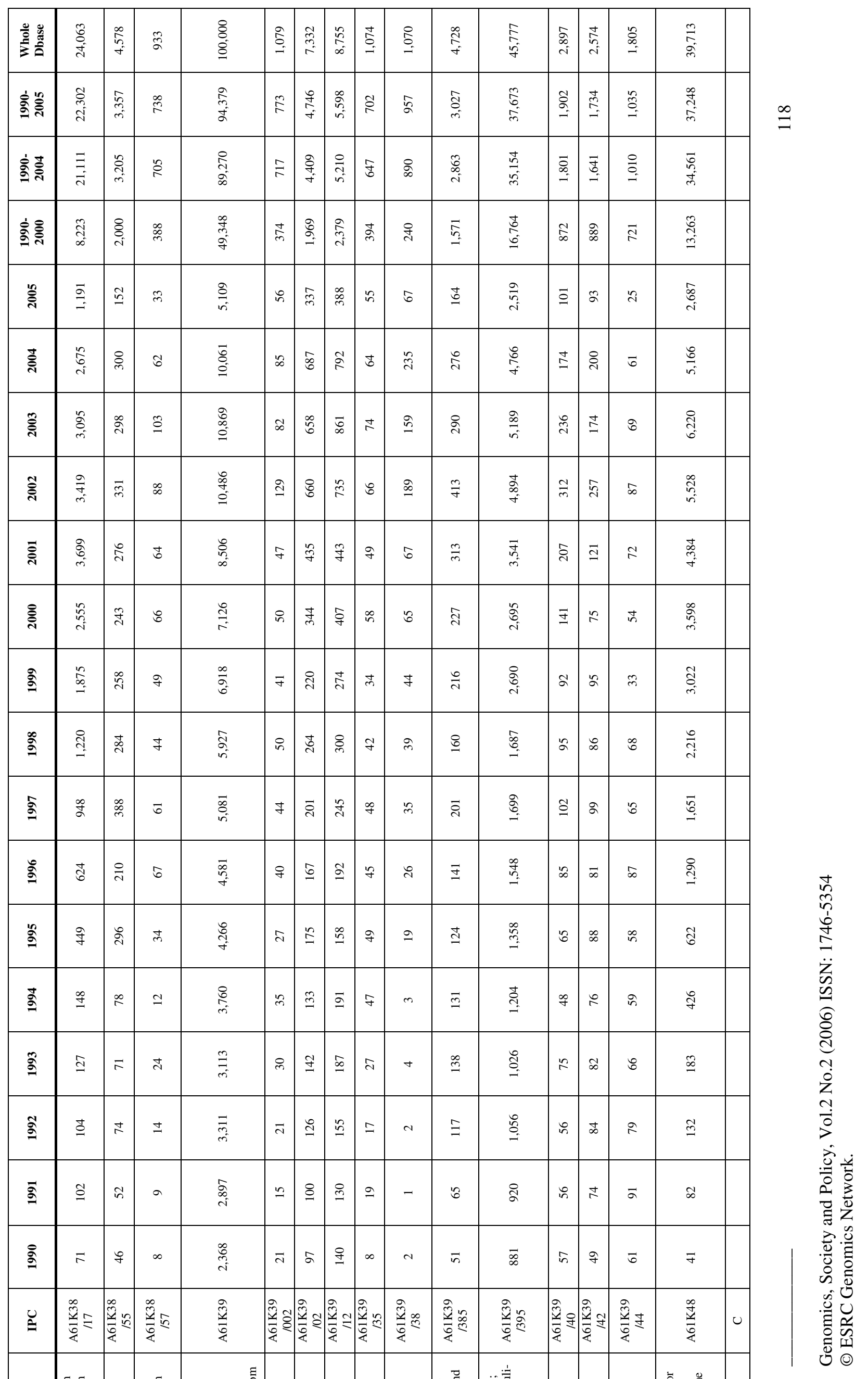

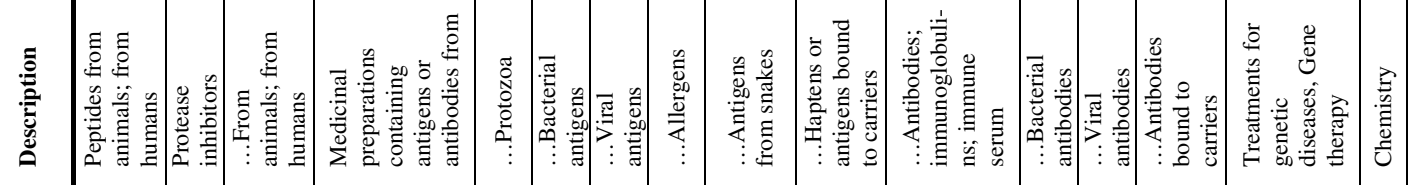




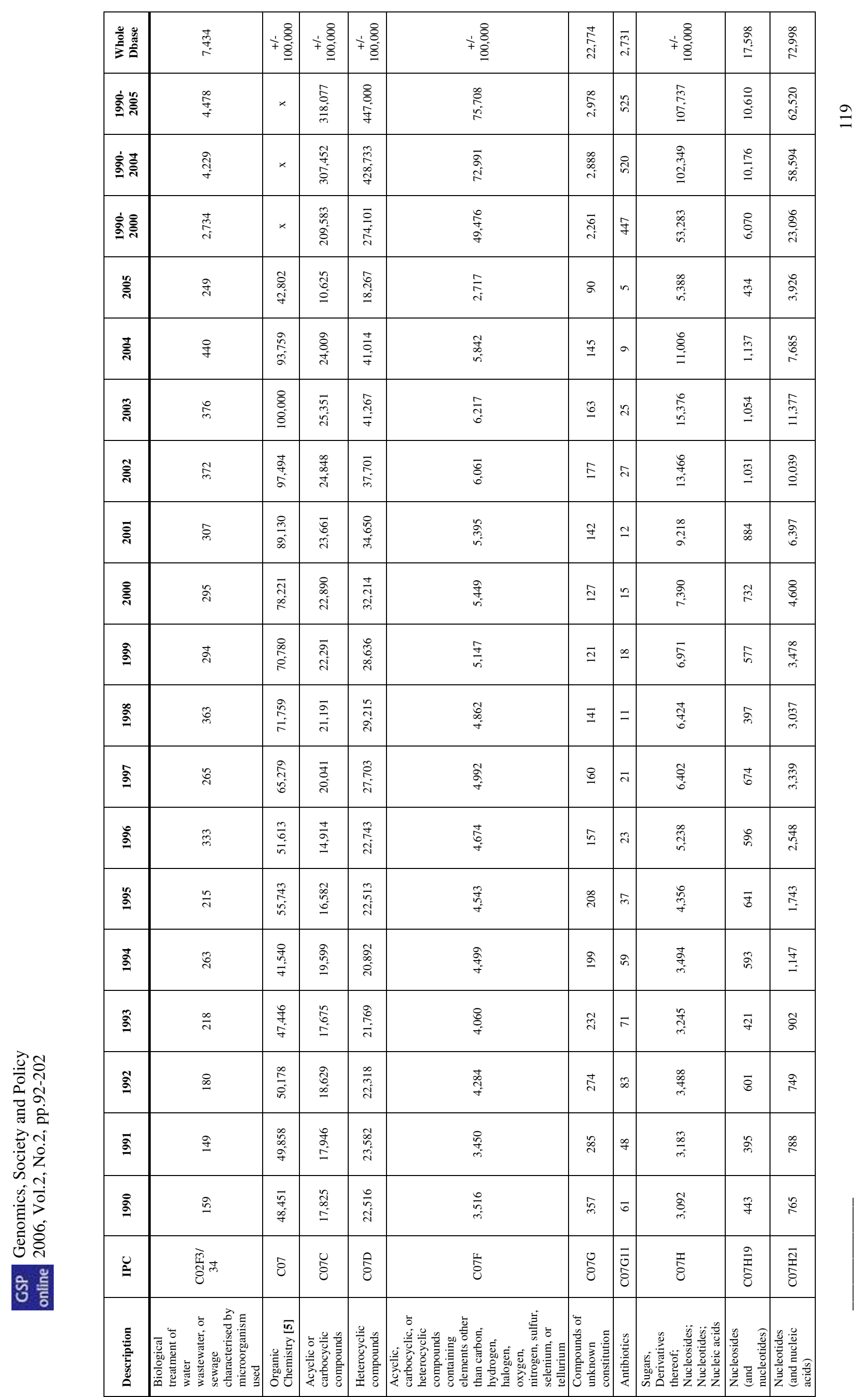




\begin{tabular}{|c|c|c|c|c|c|c|c|c|c|c|c|c|c|}
\hline 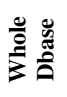 & & 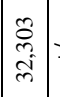 & $\left.\mid \begin{array}{ll}1 & 0 \\
+ & 0 \\
+ & 0 \\
0\end{array}\right]$ & 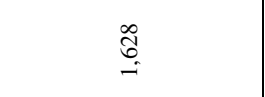 & 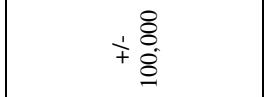 & 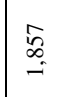 & \begin{tabular}{|l|l}
$\infty$ \\
\multirow{2}{*}{} \\
+
\end{tabular} & 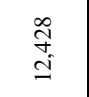 & $\underset{f}{\stackrel{f}{*}}$ & 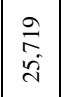 & $\begin{array}{l}\vec{t} \\
\infty \\
\vec{b}\end{array}$ & 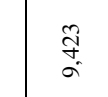 & $\begin{array}{l}\stackrel{a}{2} \\
\alpha \\
\sigma\end{array}$ \\
\hline 离 & & $\mid \begin{array}{l}9 \\
9 \\
i \\
\end{array}$ & 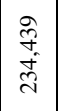 & $\stackrel{ \pm}{\underset{ \pm}{*}}$ & $\begin{array}{l}\text { D. } \\
\infty \\
\text { త్ }\end{array}$ & 胥 & 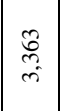 & $\stackrel{\widehat{్}}{=}$ & \& & 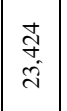 & $\begin{array}{l}\stackrel{0}{f} \\
\underset{j}{+}\end{array}$ & 总 & 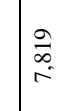 \\
\hline 言 言 & & $\begin{array}{l}\mathcal{I} \\
\mathfrak{d} \\
\text { İ }\end{array}$ & 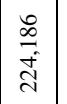 & $\stackrel{\sim}{\cong}$ & $\begin{array}{l}\text { b. } \\
\stackrel{n}{n} \\
\stackrel{\Xi}{\Xi}\end{array}$ & $\begin{array}{l}\infty \\
\stackrel{\infty}{\sim} \\
\stackrel{2}{-}\end{array}$ & 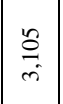 & $\begin{array}{l}\stackrel{+}{\Delta} \\
\stackrel{\Omega}{0}\end{array}$ & 怘 & $\begin{array}{l}\overline{\mathrm{d}} \\
\overline{\mathrm{d}}\end{array}$ & 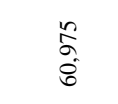 & 胥 & בֶב \\
\hline 商言 & & 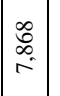 & 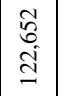 & $\stackrel{\infty}{\infty}$ & $\frac{\infty}{\vec{n}}$ & $\hat{q}$ & $\cong$ & $\begin{array}{l}\stackrel{\partial}{0} \\
i\end{array}$ & $\stackrel{\infty}{\simeq}$ & 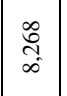 & $\begin{array}{l}\text { वे } \\
\text { ç }\end{array}$ & 兮 & $\begin{array}{l}\overrightarrow{\widehat{d}} \\
\mathrm{i}\end{array}$ \\
\hline 今్ & & $\stackrel{\infty}{\stackrel{\sigma}{\sigma}}$ & 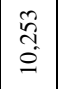 & in & $\begin{array}{l}\bar{\sigma} \\
\text { d. }\end{array}$ & 点 & $\stackrel{\infty}{\sim}$ & $\frac{m}{m}$ & m & త్ & $\begin{array}{l}n \\
\stackrel{q}{m} \\
m\end{array}$ & $\stackrel{\leftrightarrow}{\mathrm{C}}$ & $\frac{7}{6}$ \\
\hline 言 & & $\stackrel{\Xi}{\Xi}$ & 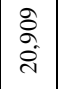 & $\Xi$ & $\begin{array}{l}\text { \& } \\
\text { I } \\
\text { I }\end{array}$ & : & iे & ర్ & ले & 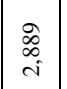 & $\begin{array}{l}a \\
\stackrel{d}{0} \\
b\end{array}$ & \& & ت্ণ্ড- \\
\hline ثิ & & 空 & $\frac{\hat{b}}{\frac{0}{20}}$ & $E$ & $\begin{array}{l}n \\
0 \\
\infty \\
n \\
n\end{array}$ & $\stackrel{\infty}{\infty}$ & gे & $\stackrel{0}{\circ}$ & $n$ & $\frac{n}{m}$ & $\stackrel{\circ}{\stackrel{\infty}{\infty}}$ & $\frac{n}{a}$ & 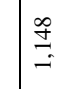 \\
\hline క్ & & $\stackrel{\stackrel{9}{=}}{=}$ & \begin{tabular}{|l}
0 \\
0 \\
0 \\
0
\end{tabular} & $\stackrel{\mathscr{\sigma}}{a}$ & $\begin{array}{l}\frac{n}{0} \\
\end{array}$ & $\stackrel{\text { ¿ }}{\sim}$ & 活 & $\begin{array}{l}\bar{F} \\
\text { i } \\
i\end{array}$ & F & $\stackrel{\circ}{\circ}$ & $\stackrel{n}{\exists}$ & 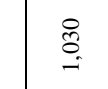 & $\stackrel{\infty}{\stackrel{2}{=}}$ \\
\hline స్ & & $\infty$ & 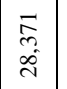 & 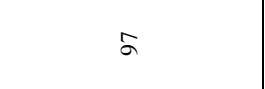 & \begin{tabular}{l}
0 \\
on \\
\multirow{J}{*}{}
\end{tabular} & $\cong$ & f & $\begin{array}{l}0 \\
\stackrel{b}{0} \\
i\end{array}$ & q & Fे & 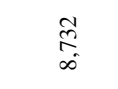 & $\vec{\infty}$ & E \\
\hline ষ্ণ & & $\mid \begin{array}{c}\infty \\
\vdots \\
- \\
-\end{array}$ & $\begin{array}{l}n \\
0 \\
6 \\
\infty \\
\infty \\
-1\end{array}$ & 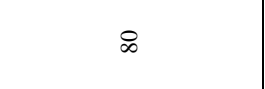 & 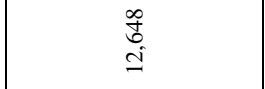 & $\sigma$ & $\overline{\tilde{e}}$ & 吕 & $\stackrel{\infty}{n}$ & 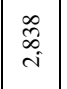 & 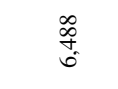 & 芯 & 索 \\
\hline$\stackrel{g}{g}$ & & 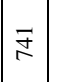 & 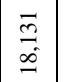 & m & $\stackrel{\mathbb{a}}{\stackrel{N}{=}}$ & 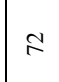 & $\stackrel{\circ}{\sim}$ & 号 & $m$ & 志 & ô. & $\underset{\sim}{\mathbb{N}}$ & 究 \\
\hline$\stackrel{\circ}{\circ}$ & & $\stackrel{\Sigma}{\Sigma}$ & \begin{tabular}{|l|}
$\vec{\infty}$ \\
$\tilde{c}_{0}^{-1}$
\end{tabular} & $\bar{\infty}$ & 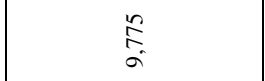 & 8 & 壳 & $\stackrel{\infty}{\stackrel{\infty}{m}}$ & $\stackrel{\sim}{\circ}$ & $\stackrel{\widetilde{m}}{\rightarrow}$ & 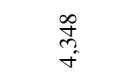 & ๙ั & 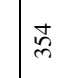 \\
\hline$\underline{\sigma}$ & & $\vec{\infty}$ & 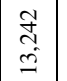 & $\bar{a}$ & 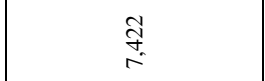 & $\infty$ & 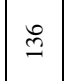 & $\ddot{m}$ & 岕 & $\vec{\Omega}$ & 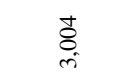 & Oి & 夺 \\
\hline ڤั & & 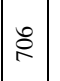 & $\mid \begin{array}{l}\mathbf{t} \\
0 \\
0 \\
0\end{array}$ & \pm & $\begin{array}{l}8 \\
0 \\
0 \\
6\end{array}$ & 8 & 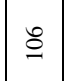 & $\vec{d}$ & $\stackrel{\infty}{0}$ & $\frac{m}{6}$ & to & 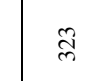 & $\tilde{\omega}$ \\
\hline$\stackrel{10}{g}$ & & $\stackrel{n}{\infty}$ & 它 & $\infty$ & 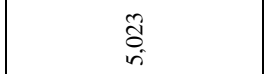 & in & $\vec{\infty}$ & \&े & ส & $\stackrel{\circ}{f}$ & 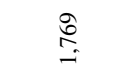 & 志 & $\underset{\widetilde{\pi}}{\infty}$ \\
\hline 奈 & & i & \begin{tabular}{|l}
$\infty$ \\
0 \\
0 \\
$\infty$ \\
$\infty$
\end{tabular} & ส & శ్ & $=$ & $\stackrel{+}{m}$ & o & $m$ & $\stackrel{\overbrace{}}{\varrho}$ & in & $\underset{ల}{\mathscr{ల}}$ & $\bar{\infty}$ \\
\hline$\stackrel{0}{g}$ & & $\stackrel{8}{\circ}$ & 点 & $\pi$ & $\approx$ & r & $\stackrel{1}{\circ}$ & in & $\mathrm{d}$ & 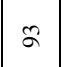 & $\ddot{n}$ & तั & g \\
\hline 亏ั & & $\frac{7}{6}$ & 总 & $m$ & 荡 & 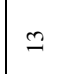 & in & $\infty$ & $m$ & $\stackrel{8}{\varrho}$ & $\underset{ల}{.}$ & 戹 & it \\
\hline $\bar{\sigma}$ & & iे & 总 & $\stackrel{d}{A}$ & $\underset{\infty}{~}$ & $\simeq$ & $\stackrel{\circ}{\circ}$ & $\stackrel{\infty}{q}$ & $a$ & 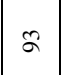 & $\stackrel{\widehat{\lambda}}{ }$ & 尔 & $\bar{\infty}$ \\
\hline$\stackrel{\circ}{2}$ & & $\begin{array}{l}\infty \\
\substack{\infty \\
\infty}\end{array}$ & $\begin{array}{l}\tilde{2} \\
\hat{\sigma} \\
0\end{array}$ & 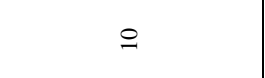 & $\stackrel{\circ}{\circ}$ & 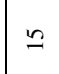 & $=$ & $\vec{n}$ & 0 & $\infty$ & 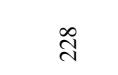 & $\stackrel{m}{\sim}$ & $\underset{q}{q}$ \\
\hline U & $\begin{array}{l}\overline{\mathbb{I}} \\
\stackrel{8}{8}\end{array}$ & 客 & 章 & 莣 & 竞 & $\frac{7}{2} \stackrel{2}{2}$ & 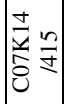 & 索哭 & 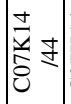 & 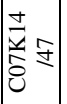 & 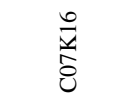 & 昱 & 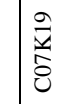 \\
\hline 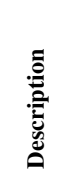 & 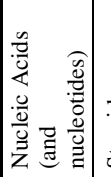 & 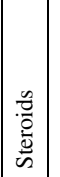 & 童 & 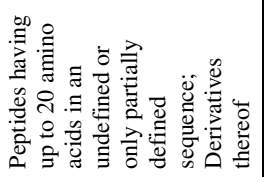 & 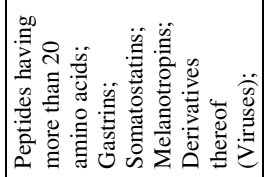 & 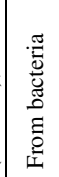 & 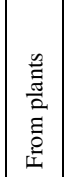 & 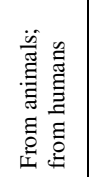 & 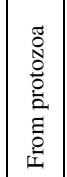 & 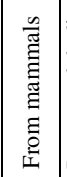 & 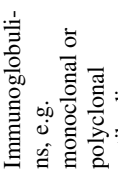 & 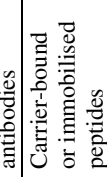 & 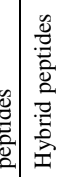 \\
\hline
\end{tabular}




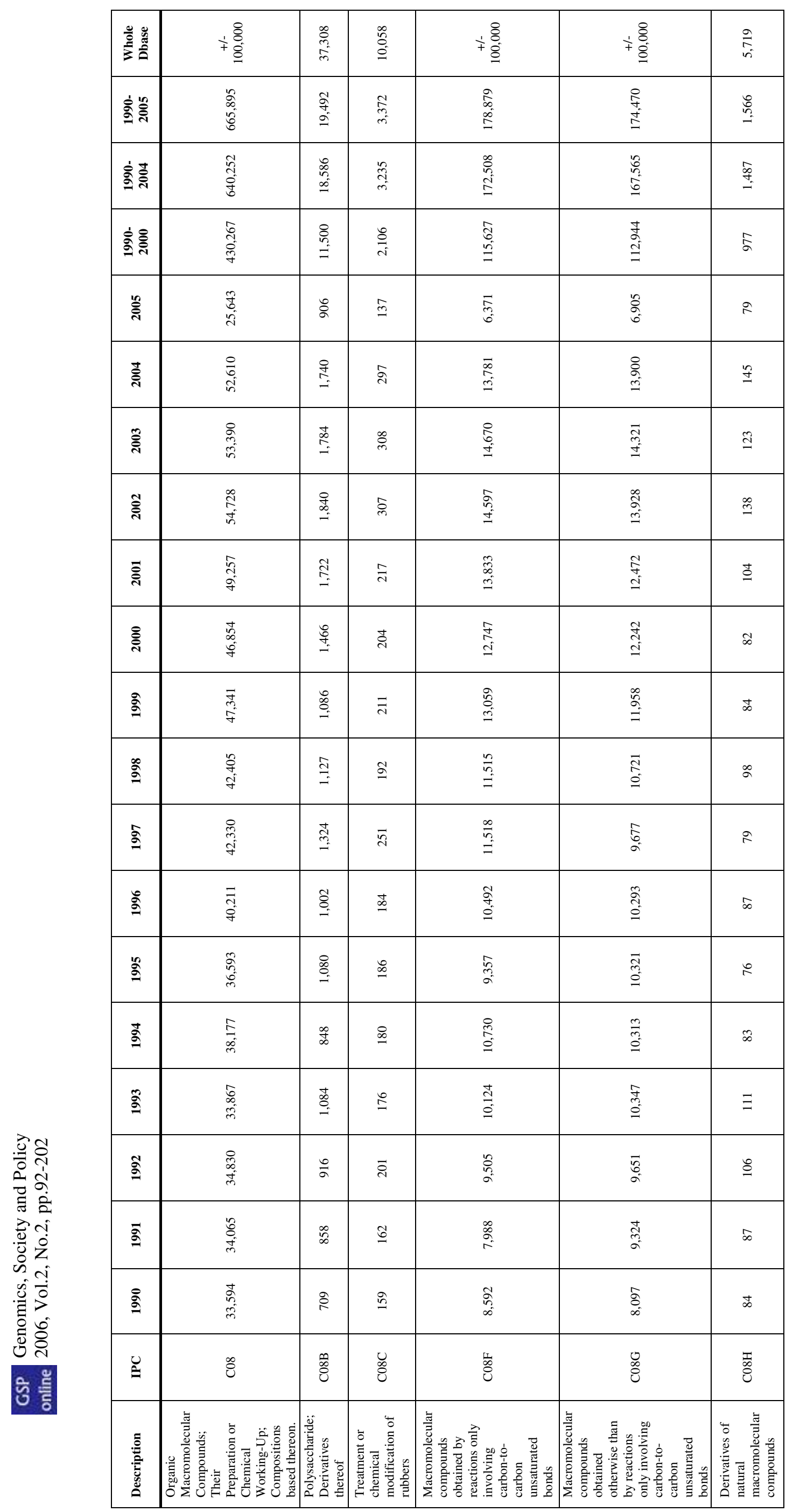




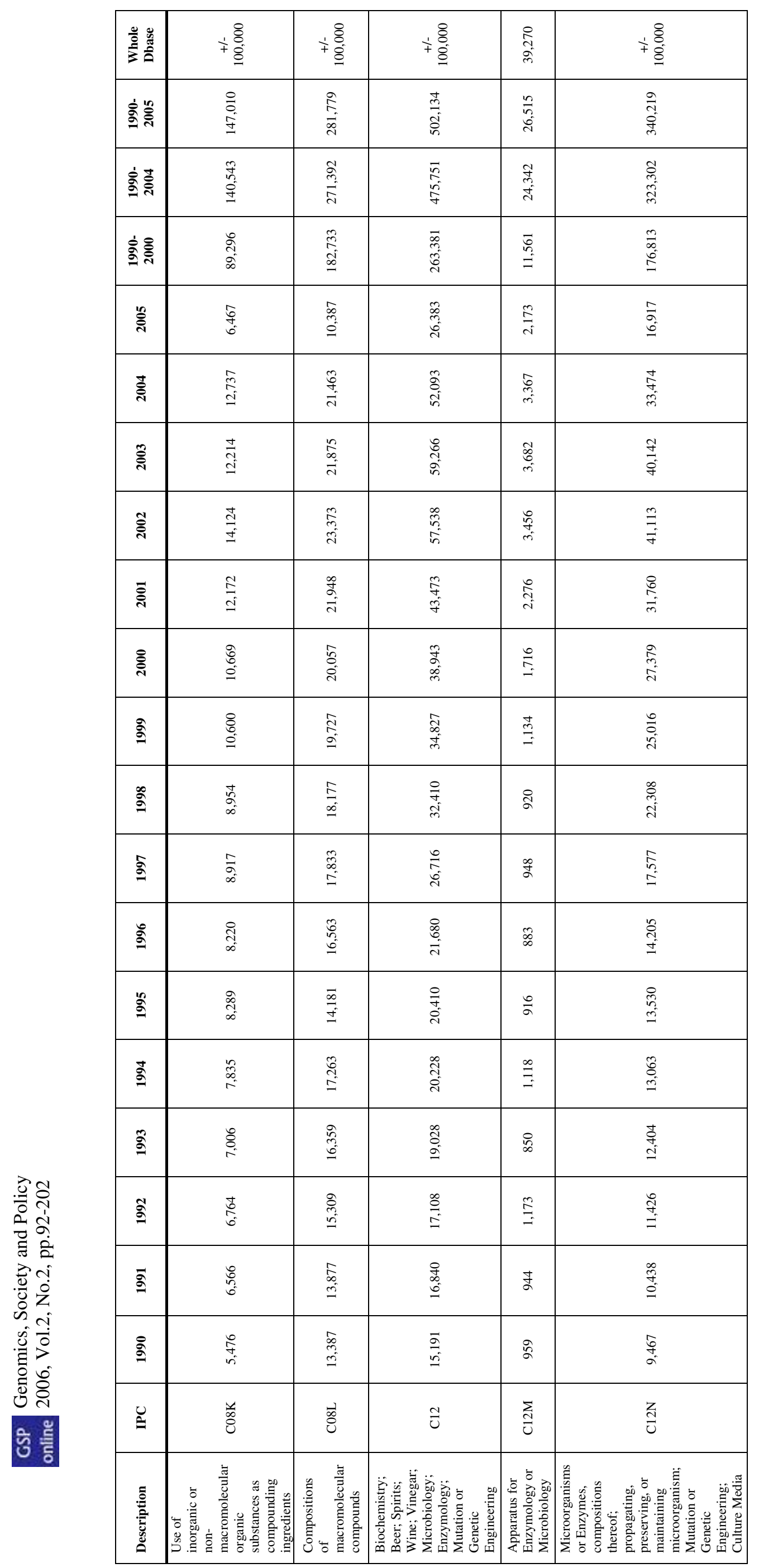




\begin{tabular}{|c|c|c|c|c|c|c|c|}
\hline 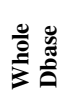 & 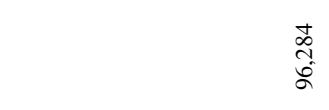 & $\underset{-}{\stackrel{2}{0}}$ & $\underset{\substack{\infty \\
\stackrel{0}{i}}}{\stackrel{\infty}{i}}$ & 季 & 总 & $\begin{array}{l}8 \\
+ \\
+ \\
+\end{array}$ & 品 \\
\hline 紊譶 & $\begin{array}{l}\overrightarrow{\overrightarrow{0}} \\
\stackrel{2}{1}\end{array}$ & 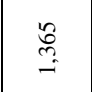 & 高 & $\begin{array}{l}\underset{f}{+} \\
\underset{i}{i}\end{array}$ & 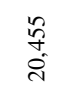 & 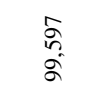 & 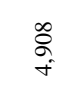 \\
\hline 言喜 & 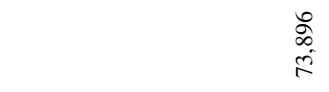 & $\overrightarrow{\mathrm{c}}$ & 章 & $\begin{array}{l}+ \\
\text { ते } \\
\text { in }\end{array}$ & $\begin{array}{l}\stackrel{0}{0} \\
\stackrel{0}{0}\end{array}$ & 筞 & 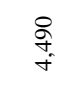 \\
\hline 章言 & $\begin{array}{l}\infty \\
\substack{+\gamma \\
\gamma}\end{array}$ & $\stackrel{\circ}{\circledR}$ & $\begin{array}{l}\text { for } \\
\text { of } \\
\text { d }\end{array}$ & 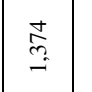 & 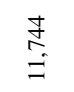 & 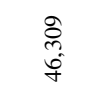 & $\overline{\bar{i}}$ \\
\hline 䓂 & 点 & t & $\bar{\sim}$ & $\ddot{9}$ & $\ddot{\infty}$ & $\begin{array}{l}\text { 䯧 } \\
\text { q }\end{array}$ & $\stackrel{\infty}{\not}$ \\
\hline 熟 & 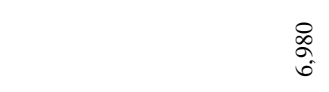 & 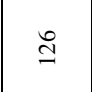 & 专 & 志 & 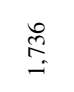 & 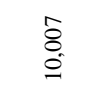 & $\tilde{E}$ \\
\hline 密 & $\underset{\infty}{\infty}$ & $\stackrel{n}{=}$ & 尛 & 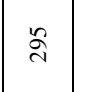 & $\stackrel{\ominus}{\stackrel{i}{i}}$ & $\begin{array}{l}\overline{\bar{y}} \\
\underline{\underline{y}}\end{array}$ & $\underset{\infty}{ \pm}$ \\
\hline స్ & $\begin{array}{l}\text { 素 } \\
\text { 足 }\end{array}$ & of & 字 & ते & 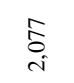 & 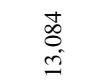 & 号 \\
\hline ప్ & 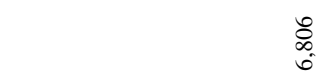 & $\stackrel{\infty}{\Xi}$ & 然 & $\cong$ & $\stackrel{\circ}{\stackrel{0}{0}}$ & 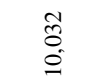 & $\stackrel{8}{\infty}$ \\
\hline 高 & $\begin{array}{c}\text { qu } \\
\text { on } \\
\text { in }\end{array}$ & s. & 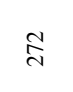 & $\stackrel{\cong}{\cong}$ & $\stackrel{\circ}{\stackrel{2}{\leftrightarrows}}$ & $\begin{array}{l}\infty \\
\underset{\infty}{\infty}\end{array}$ & 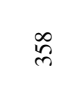 \\
\hline gे & 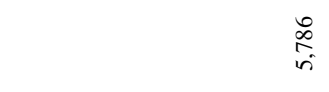 & 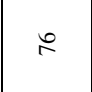 & 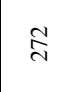 & 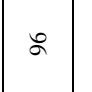 & $\stackrel{\infty}{\overparen{్}}$ & 祃 & $\stackrel{\infty}{p}$ \\
\hline g & $\begin{array}{l}8 \\
8 \\
8 \\
8\end{array}$ & $\therefore$ & $\stackrel{\circ}{\circledR}$ & $\Xi$ & $\aleph_{\sigma}^{\infty}$ & $\begin{array}{l}\infty \\
0 \\
0 \\
0\end{array}$ & $\stackrel{\Omega}{\Omega}$ \\
\hline$\underline{\underline{a}}$ & $\begin{array}{c}\infty \\
\substack{q \\
⿱}\end{array}$ & $\infty$ & ลे & 9 & $\stackrel{m}{\Xi}$ & $\begin{array}{c}8 \\
\frac{8}{7} \\
7\end{array}$ & bे \\
\hline$\stackrel{\circ}{\circ}$ & 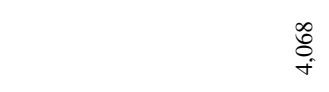 & 8 & g d & $\Xi$ & $\overbrace{\infty}^{\infty}$ & 号 & $£$ \\
\hline gू & : & $\stackrel{\infty}{\infty}$ & 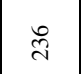 & 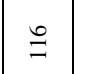 & $\supseteqq$ & 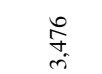 & $\stackrel{\infty}{\cong}$ \\
\hline 壴 & శ్ & $\infty$ & $\stackrel{\circ}{N}$ & $\stackrel{8}{6}$ & ๙ి & 然 & 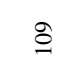 \\
\hline $\mathrm{g}$ & $\frac{\widetilde{m}}{m}$ & $\ddot{8}$ & 5 & $\underline{m}$ & $\stackrel{8}{8}$ & 尊 & $\overline{\mathrm{I}}$ \\
\hline 亏ี & 兽 & in & 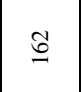 & $\cong$ & $\mathscr{8}$ & $\frac{\infty}{i}$ & 우 \\
\hline $\bar{\Xi}$ & 总 & $\tilde{n}$ & $\stackrel{\circ}{\underline{0}}$ & $\stackrel{\circ}{g}$ & $\infty_{\infty}^{\infty}$ & $\frac{\bar{g}}{\mathrm{~s}}$ & $\underset{I}{q}$ \\
\hline ळे & 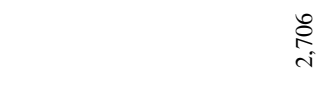 & o & $\stackrel{8}{8}$ & $\cong$ & 志 & 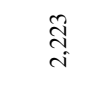 & $\exists$ \\
\hline$\cong$ & $\underset{\mathrm{d}}{\overline{\mathrm{d}}}$ & $\underset{\bar{z}}{\bar{u}} \beth$ & $\underset{\bar{z}}{\bar{u}} \pm$ & 言 & 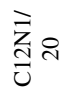 & 莺 & 穿す \\
\hline 害 & 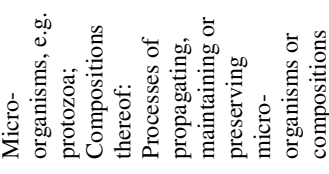 & 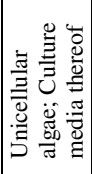 & 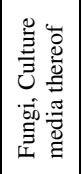 & 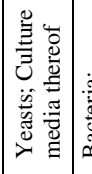 & 童 & 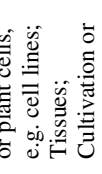 & 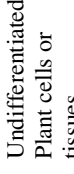 \\
\hline
\end{tabular}




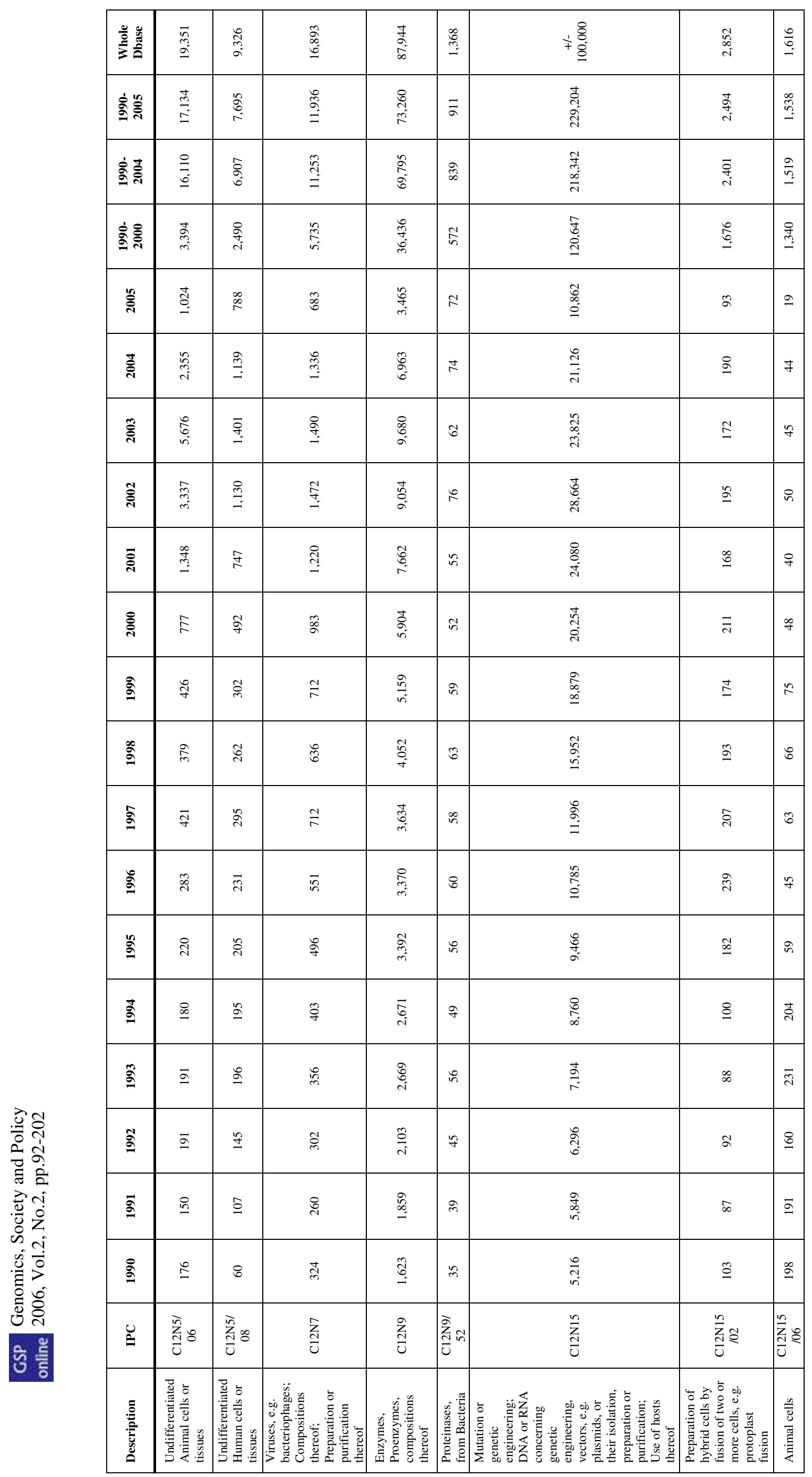




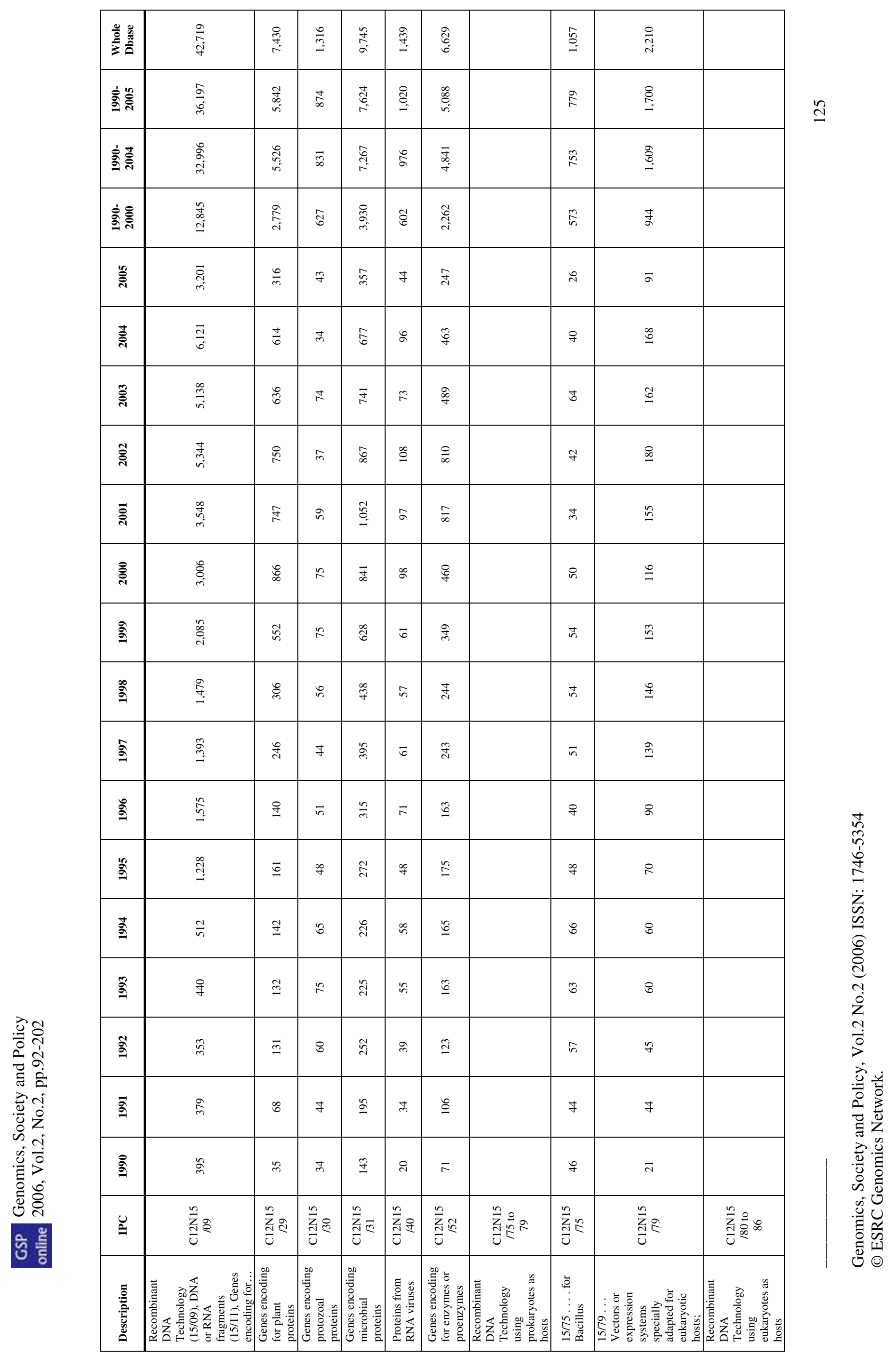




\begin{tabular}{|c|c|c|c|c|c|c|c|c|c|c|c|c|}
\hline 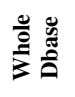 & 窵 & $\begin{array}{l}\tilde{y} \\
\text { co } \\
\sim\end{array}$ & $\begin{array}{l}\frac{J}{E} \\
\stackrel{2}{2}\end{array}$ & $\begin{array}{l}\stackrel{0}{0} \\
\stackrel{8}{\mathrm{I}} \\
\mathrm{i}\end{array}$ & 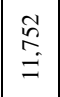 & $\begin{array}{r}8 \\
+\frac{8}{8} \\
\dot{0}\end{array}$ & 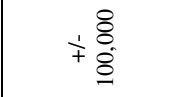 & $\frac{5}{a}$ & $\stackrel{8}{S}$ & go & $\frac{8}{\infty}$ & 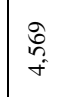 \\
\hline 亏ેे & $\stackrel{\circ}{\circ}$ & $\begin{array}{l}\bar{B} \\
\text { n. } \\
\text { in }\end{array}$ & $\begin{array}{l}\hat{O} \\
\infty \\
\stackrel{0}{0}\end{array}$ & $\begin{array}{l}\tilde{\sigma} \\
\text { o. }\end{array}$ & $\begin{array}{l}\infty \\
\infty \\
\infty \\
\alpha \\
\alpha\end{array}$ & $\begin{array}{l}\overrightarrow{\widehat{B}} \\
\vec{j} \\
\stackrel{j}{3}\end{array}$ & $\begin{array}{l}\stackrel{8}{+} \\
\stackrel{+}{0} \\
-1\end{array}$ & $\underset{\substack{\text { f } \\
\text { d }}}{ }$ & $\stackrel{\frac{a}{m}}{=}$ & 8̊ & $\underset{-}{\stackrel{*}{*}}$ & $\frac{\infty}{m}$ \\
\hline 㝘 & $\bar{\jmath}$ & 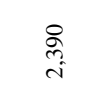 & $\begin{array}{l}\text { 象 } \\
\text { م. }\end{array}$ & 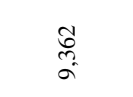 & $\begin{array}{l}\overrightarrow{5} \\
\infty \\
\infty\end{array}$ & 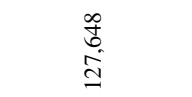 & 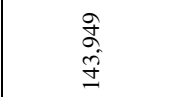 & $\begin{array}{l}\infty \\
\infty \\
\dot{\infty} \\
\dot{m}\end{array}$ & ब্ণ & 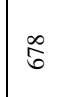 & $\vec{n}$ & 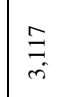 \\
\hline 亏ેे & $\stackrel{\infty}{\infty}$ & $\widehat{C}_{-}^{\infty}$ & 卓 & $\begin{array}{l}\stackrel{\infty}{0} \\
\stackrel{m}{f}\end{array}$ & $\begin{array}{l}\frac{n}{n} \\
\frac{n}{f}\end{array}$ & $\begin{array}{l}\hat{a} \\
\text { s. }\end{array}$ & 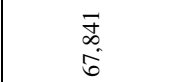 & $\begin{array}{l}\stackrel{\infty}{ \pm} \\
\stackrel{j}{+}\end{array}$ & S̆ & 奇 & $\stackrel{\infty}{\stackrel{-}{二}}$ & $\stackrel{m}{i}$ \\
\hline 岕 & in & $\Xi$ & 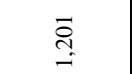 & $\frac{0}{6}$ & ì & $\begin{array}{c}\mathscr{\infty} \\
\stackrel{\alpha}{\sigma}\end{array}$ & $\underset{\overrightarrow{\mathrm{d}}}{\overline{\mathrm{g}}}$ & $\hat{0}$ & $\vec{\lambda}$ & $\simeq$ & $\ddot{\lambda}$ & $\approx$ \\
\hline ఫ্ড & $\stackrel{+}{\infty}$ & $\Xi$ & $\stackrel{9}{i}$ & $\stackrel{\mathscr{O}}{\stackrel{\infty}{-}}$ & హి & 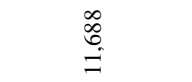 & $\begin{array}{l}\overrightarrow{0} \\
\text { b. } \\
\text { on }\end{array}$ & 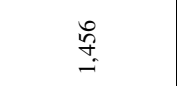 & $\stackrel{i}{n}$ & i & $\approx$ & $\stackrel{2}{ }$ \\
\hline ڤ్సે & $\approx$ & के & $\stackrel{\infty}{\underset{i}{\vec{j}}}$ & $\stackrel{\vec{\Phi}}{-}$ & : & 胥 & $\begin{array}{l}\text { D. } \\
\text { an } \\
\end{array}$ & $\underset{-\underset{-}{\sim}}{\mathbb{\infty}}$ & 8 & $\tilde{m}$ & $\infty$ & f \\
\hline స్ํำ & $\$$ & ন্ป & 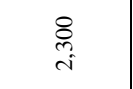 & $\stackrel{\infty}{=}$ & $\stackrel{\stackrel{\circ}{g}}{=}$ & $\begin{array}{l}\infty \\
\vdots \\
\\
\end{array}$ & $\begin{array}{l}\overline{\mathbf{D}} \\
\stackrel{\sim}{0} \\
\stackrel{i}{0}\end{array}$ & $\frac{\pi}{\mathrm{d}}$ & $\infty$ & $F$ & $\approx$ & $\vec{a}$ \\
\hline ప్ & $\Phi$ & $\underline{g}$ & $\underset{\substack{ \pm i}}{\vec{J}}$ & 号 & 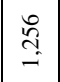 & 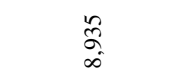 & 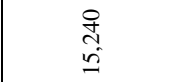 & $\stackrel{\infty}{\stackrel{\infty}{\sim}}$ & $\infty$ & $\bar{n}$ & $\tilde{b}$ & $\Xi$ \\
\hline ث્సે & $\approx$ & $\bar{i}$ & 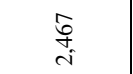 & Sू & \% & $\begin{array}{l}\infty \\
\vdots \\
\infty \\
\infty\end{array}$ & $\begin{array}{l}\tilde{N} \\
\text { o. } \\
\text { తa }\end{array}$ & तี & I & F & $\stackrel{+}{\infty}$ & 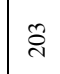 \\
\hline$\stackrel{\partial}{\partial}$ & 5 & $\stackrel{\sim}{\circ}$ & $\stackrel{\infty}{\stackrel{\infty}{n}}$ & $\underset{\infty}{\mathbb{\infty}}$ & \& & $\begin{array}{l}\stackrel{m}{0} \\
\tilde{o}_{\infty}\end{array}$ & $\begin{array}{l}\underset{ \pm}{ \pm} \\
=\end{array}$ & 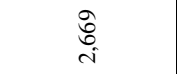 & $\approx$ & $\infty$ & 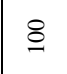 & ন্ন \\
\hline$\stackrel{\infty}{\sigma}$ & 8 & I & ڤे & 志 & 过 & 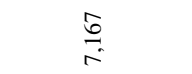 & $\frac{\stackrel{\infty}{a}}{\sigma^{\prime}}$ & $\begin{array}{l}\vec{n} \\
i\end{array}$ & a & in & $\stackrel{g}{\varrho}$ & ্ֻণ \\
\hline$\underline{\sigma}$ & 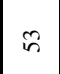 & $\underline{\square}$ & $\bar{\sigma}$ & in & in & 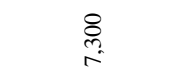 & तี & 兽 & $\stackrel{\circ}{ }$ & $\stackrel{\circ}{n}$ & $\stackrel{\infty}{=}$ & 占 \\
\hline$\stackrel{\circ}{\circ}$ & t & $\stackrel{\infty}{=}$ & ల్m & \&্ల & 专 & 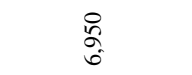 & 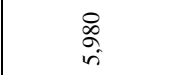 & $\underset{\substack{0 \\
i \\
i}}{ }$ & $\cong$ & $\stackrel{\infty}{q}$ & 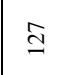 & $\stackrel{t}{N}$ \\
\hline$\stackrel{\circ}{\circ}$ & $\approx$ & 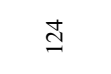 & 总 & 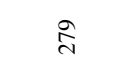 & $\stackrel{\infty}{i}$ & $\begin{array}{l}\frac{n}{7} \\
f \\
0\end{array}$ & 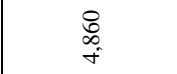 & $\underset{i}{\stackrel{n}{i}}$ & $\stackrel{\varrho}{=}$ & $F$ & $\cong$ & ते \\
\hline J & in & 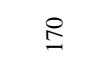 & ๙ิे & สี & $\stackrel{\infty}{ }$ & 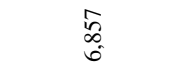 & 卓 & $\begin{array}{l}\text { సे } \\
\text { ì }\end{array}$ & \pm & F & $\Xi$ & 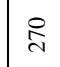 \\
\hline$\stackrel{\Omega}{\sigma}$ & fo & $\hat{n}$ & $\underset{\text { I }}{2}$ & $\cong$ & 黑 & 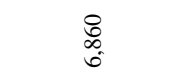 & $\underset{c}{f}$ & \begin{tabular}{l}
$\Delta$ \\
\multirow{2}{*}{}
\end{tabular} & $\sigma$ & $F$ & 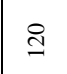 & 吉 \\
\hline$\delta$ & $\bar{n}$ & 吉 & $\Xi$ & I & I & $\begin{array}{l}\cong \\
0 \\
0\end{array}$ & के & $\stackrel{\text { సે }}{=}$ & t & $\bar{\lambda}$ & $\stackrel{\circ}{\circ}$ & \& \\
\hline$\overline{\mathrm{g}}$ & $\bar{\lambda}$ & $\stackrel{\check{g}}{\Omega}$ & $\overrightarrow{\underline{m}}$ & I & $\stackrel{\varrho}{\varrho}$ & $\frac{0}{0 .}$ & $\begin{array}{l}\text { à } \\
\text { à }\end{array}$ & $\underset{-}{S}$ & $\vec{n}$ & ส & 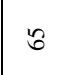 & $\bar{\sigma}$ \\
\hline 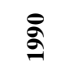 & $\stackrel{i}{\circ}$ & $\stackrel{\vartheta}{=}$ & in & 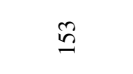 & \& & $\begin{array}{l}\text { aे } \\
\text { in }\end{array}$ & 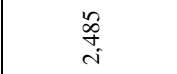 & त्̀ & 8 & ते & $\stackrel{2}{2}$ & $\stackrel{ \pm}{\underline{a}}$ \\
\hline$\stackrel{\varrho}{\varrho}$ & $\sum_{\substack{n \\
0}}^{\infty}$ & $\stackrel{a}{u} \bar{\infty}^{n}$ & 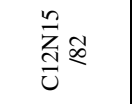 & 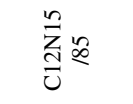 & $\sum_{\substack{n \\
0}} \infty$ & בิ & d & $\stackrel{\approx}{\tilde{U}}$ & & $\frac{\vec{z}}{d}$ & 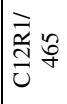 & 穿 \\
\hline 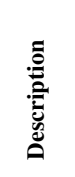 & 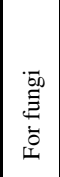 & 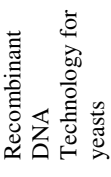 & 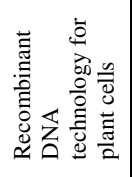 & 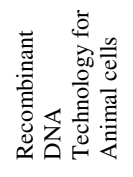 & 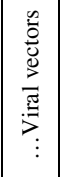 & 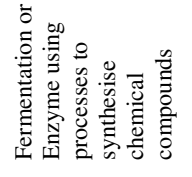 & 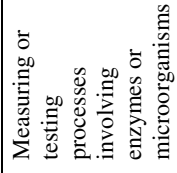 & 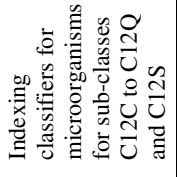 & 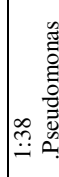 & 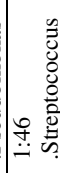 & 离 & 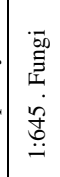 \\
\hline
\end{tabular}




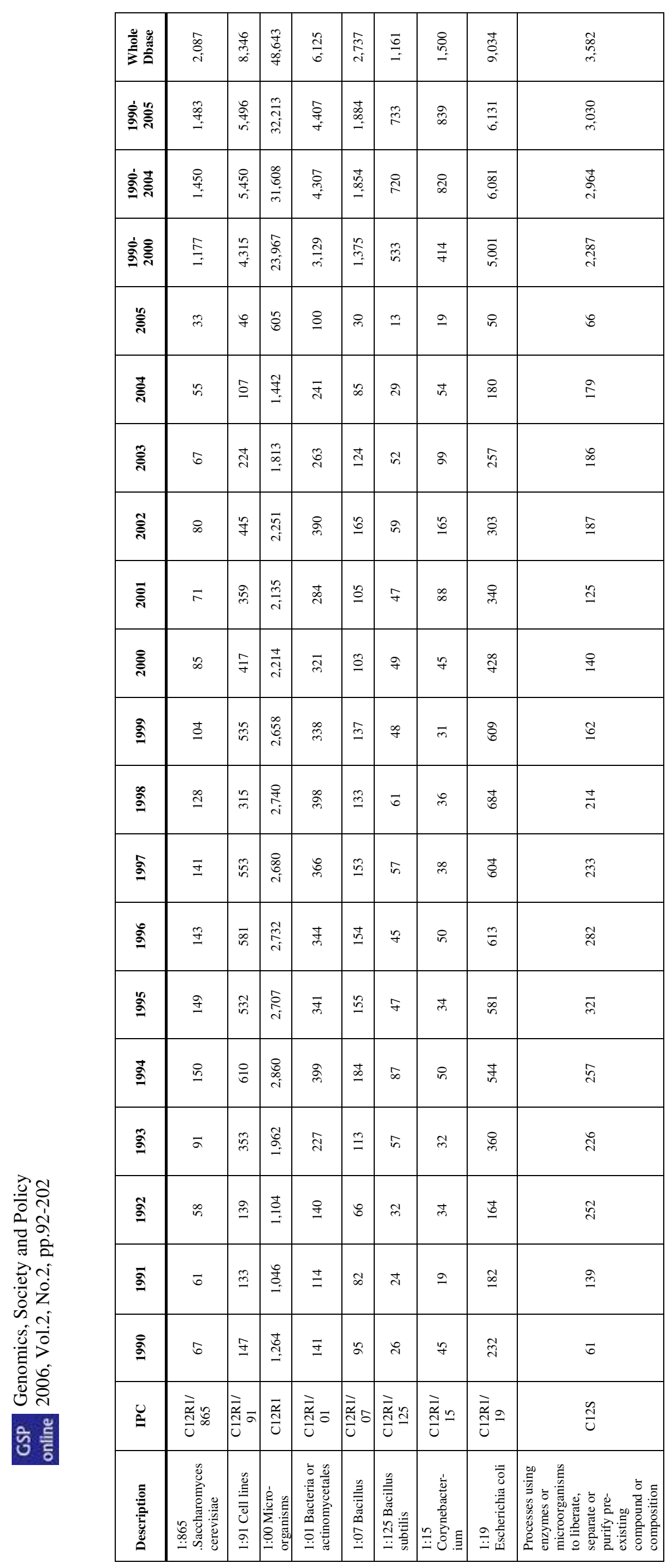




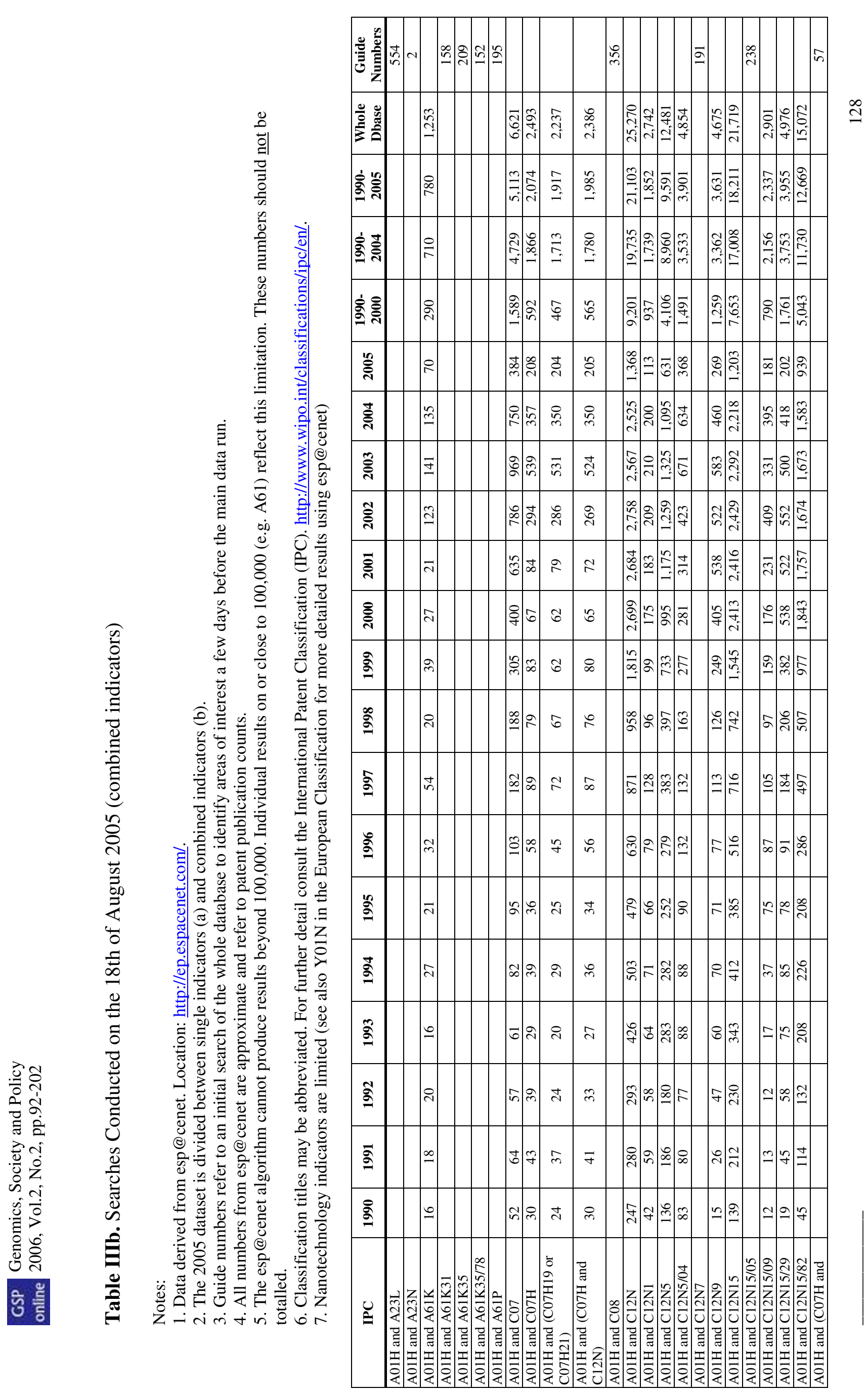

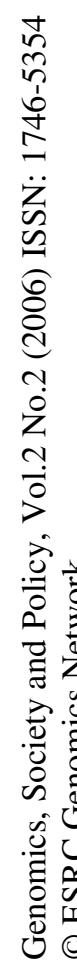




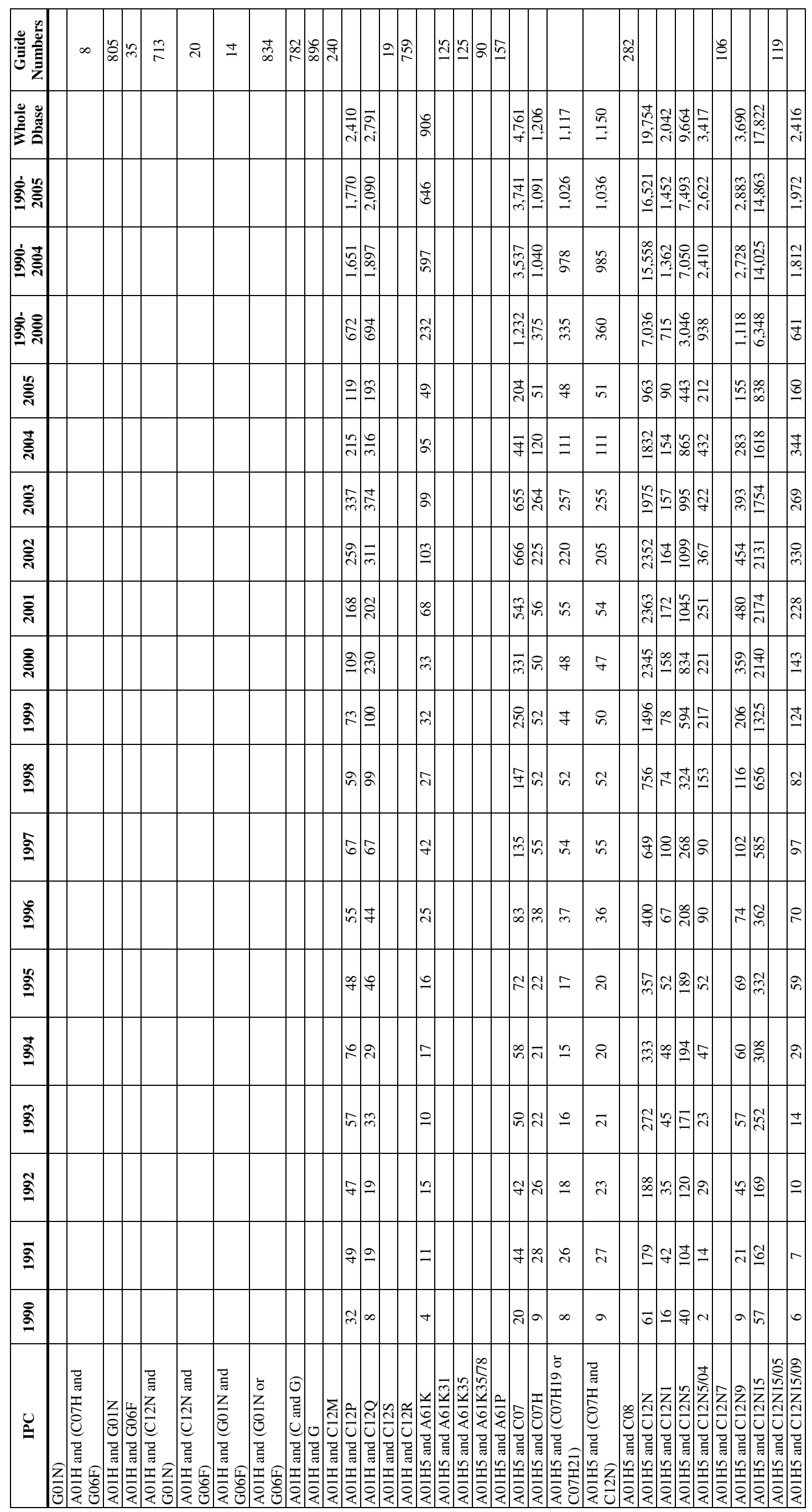




\begin{tabular}{|c|c|c|c|c|c|c|c|c|c|c|c|c|c|c|c|c|c|c|c|c|c|c|c|c|}
\hline 象 & & f & & $\infty$ & & & & 0 & 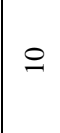 & $\overline{8}$ & 6 & & & $\cong-$ & & & & & $\hat{\imath}$ & & & & & \\
\hline 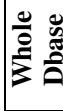 & $\mid$\begin{tabular}{lll}
0 \\
0 \\
\hdashline
\end{tabular} & & & & 8 & 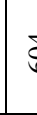 & & & & & & & $\hat{\imath}$ & & 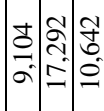 & $\begin{array}{r}8 \\
+ \\
+ \\
\\
0\end{array}$ & a & & $\mid$ & 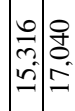 & $\stackrel{\substack{\infty \\
i}}{\stackrel{\infty}{\infty}}$ & $\mid$ & 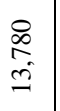 & Ĭ \\
\hline ถ્રે & 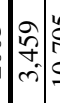 & & & & $\frac{1}{b}$ & 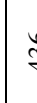 & & & & & & & 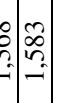 & & 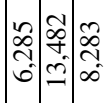 & $\frac{\tilde{c}}{\tilde{q}}$ & 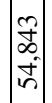 & 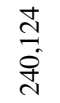 & 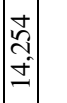 & 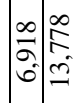 & $\begin{array}{l}\infty \\
\infty \\
\infty \\
i\end{array}$ & वे. & $\begin{array}{l}\hat{\sigma} \\
\sigma^{\prime}\end{array}$ & I \\
\hline 今े & 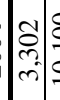 & & & & કેશ & F & & & & & & & $=$\begin{tabular}{lll}
0 \\
\hdashline \\
\hdashline
\end{tabular} & & 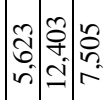 & \begin{tabular}{|l} 
Dे \\
h. \\
id
\end{tabular} & $\left|\begin{array}{|}\overline{2} \\
\overline{2} \\
\text { in }\end{array}\right|$ & $\begin{array}{l}\text { ơ } \\
\text { aें } \\
\text { ì }\end{array}$ & 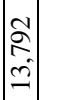 & 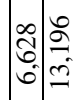 & $\frac{n}{0}$ & $\left|\begin{array}{l}0 \\
\vdots \\
\text { in }\end{array}\right|$ & $\begin{array}{l}\tilde{n} \\
\hat{n} \\
\sigma^{2}\end{array}$ & $\mathscr{d}$ \\
\hline 今े & 窟 & & & & . & 5 & & & & & & & 8 & & 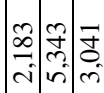 & $\mid \begin{array}{l} \pm \\
0 \\
0 \\
\infty \\
\infty \\
\infty\end{array}$ & 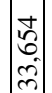 & $\begin{array}{l}\hat{\infty} \\
\hat{n}^{2} \\
\hat{n}\end{array}$ & $\left|\begin{array}{c}0 \\
0 \\
0 \\
2 \\
2\end{array}\right|$ & 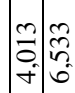 & 守 & 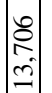 & $\begin{array}{l}\text { a } \\
\text { o. } \\
i\end{array}$ & f \\
\hline ثิे & in & & & & ते & $y$ & & & & & & & 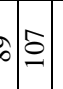 & & 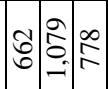 & $\begin{array}{l}0 \\
\tilde{\sigma} \\
\text { g్ }\end{array}$ & $\left|\begin{array}{l}n \\
\vdots \\
\text { in }\end{array}\right|$ & $\begin{array}{l}\stackrel{+}{\circ} \\
\stackrel{0}{=}\end{array}$ & 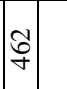 & 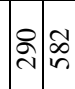 & $\frac{n}{2}$ & . & 宗 & is \\
\hline ঙั๋ & 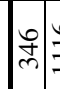 & & & & 8 & 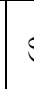 & & & & & & & $\stackrel{c}{c}$ & & 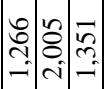 & $\frac{\vec{n}}{\vec{n}}$ & 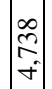 & $\begin{array}{l}\stackrel{4}{a} \\
\text { İ }\end{array}$ & $\mid$ & : & 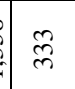 & $\mid$ & $\tilde{\alpha}$ & $\approx$ \\
\hline 气ิે & $\ddot{\sigma})$ & & & & $\infty$ & 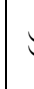 & & & & & & & 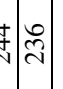 & & : & $\frac{\vec{g}}{\vec{m}}$ & 放 & $\begin{array}{l}\text { ত̃ } \\
\text { +̇ }\end{array}$ & $\stackrel{2}{2}$ & $\stackrel{n}{\sim} \underset{\substack{\infty \\
=}}{=}$ & $=\infty$ & $\mid$ & $\stackrel{\infty}{=}$ & 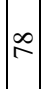 \\
\hline ङ్సิ & $\because \frac{0}{n}$ & & & & $\approx=$ & $y$ & & & & & & & ㅇำ & & 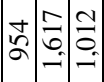 & $\begin{array}{l}\hat{a} \\
\hat{\alpha} \\
\text { do }\end{array}$ & $\left|\begin{array}{l}\infty \\
\hdashline \\
\hdashline \\
\dot{f}\end{array}\right|$ & $\frac{n}{n}$ & $\mid$ & त & $\stackrel{\infty}{\lesssim}$ & $\left|\begin{array}{l}0 \\
\text { in }\end{array}\right|$ & $\stackrel{\curvearrowright}{\infty}$ & in \\
\hline ฮิ่ & $\frac{7}{3}$ & & & & 8 & 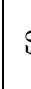 & & & & & & & \pm & & సิ & $\begin{array}{l}\mathscr{a} \\
0 \\
\text { and } \\
\text { in }\end{array}$ & $\begin{array}{l}\overrightarrow{\tilde{m}} \\
\stackrel{\sim}{+}\end{array}$ & $\begin{array}{l}\frac{J}{0} \\
\text { Q }\end{array}$ & $\mid$ & $\stackrel{\infty}{n} \underset{\sim}{\stackrel{f}{n}}$ & $\stackrel{I}{I}$ & $\mid$ & $\tilde{\infty}$ & मे \\
\hline ఫ్సి & $\bar{\sigma}$ & & & & $\infty>$ & i & & & & & & & : & & 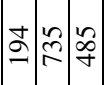 & $\begin{array}{l}\overline{\alpha o} \\
\text { di } \\
\text { in }\end{array}$ & $\left|\begin{array}{l}\vec{a} \\
\vec{s} \\
\dot{f}\end{array}\right|$ & $\stackrel{\text { c}}{\stackrel{\text { s}}{=}}$ & : & in: & $\stackrel{2}{\exists}$ & | & त్రి & $\stackrel{c}{\infty}$ \\
\hline$\hat{\sigma}$ & ले & & & & $f \circ$ & 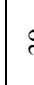 & s & & & & & & 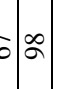 & & 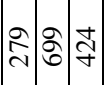 & $\mid$ & 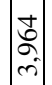 & 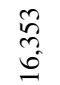 & $\left|\begin{array}{l}\infty \\
\infty \\
\infty\end{array}\right|$ & $\vec{f}$ & 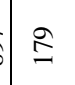 & : & $\underset{n}{ \pm}$ & i \\
\hline$\stackrel{2}{\circ}$ & 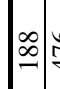 & & & & C. & ๙ & & & & & & & 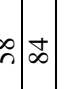 & & 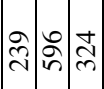 & $\begin{array}{l}\tilde{\delta} \\
\frac{\tilde{d}}{0}\end{array}$ & 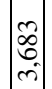 & $\stackrel{\hat{\Xi}}{\underline{n}}$ & $\frac{7}{2}$ & ๗ે) & 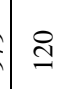 & : & 学 & $f$ \\
\hline$\hat{\sigma}$ & 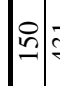 & & & & $=0$ & S & & & & & & & 5 & & 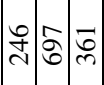 & $\begin{array}{l}\infty \\
\tilde{0} \\
2 \\
2\end{array}$ & $\mid$ & $\begin{array}{l}\infty \\
\dot{\infty} \\
\dot{J}\end{array}$ & $\partial$ & 宇: & $\stackrel{\circ}{\sim}$ & : & $\frac{\infty}{\infty}$ & i \\
\hline$\stackrel{2}{2}$ & $\infty$ & & & & 00 & $\circ$ & & & & & & & $\infty$ & & ఫ্ণ & $\begin{array}{l}\tilde{n} \\
\infty \\
\infty \\
\infty \\
a\end{array}$ & $\mid$\begin{tabular}{c|c}
$\overrightarrow{\vec{n}}$ \\
$\substack{n \\
m}$
\end{tabular} & $\stackrel{m}{m}$ & $\overrightarrow{\tilde{\infty}}$ & $f \stackrel{\infty}{\stackrel{2}{n}}$ & $\stackrel{I}{2}$ & : & $\stackrel{\infty}{\curvearrowright}$ & is \\
\hline 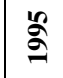 & $\because$ & & & & 0.0 & 5 & b & & & & & & $\approx$ & & 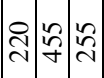 & $\begin{array}{l}n \\
n \\
n \\
= \\
=\end{array}$ & $\mid$ & $\begin{array}{l}\text { aे } \\
0 \\
\text { i }\end{array}$ & : & $\begin{array}{l}\infty \\
\stackrel{f}{*}\end{array}$ & $\stackrel{\infty}{0}$ & : & हे & is \\
\hline పे & $\pi \leqq$ & & & & ro & 4 & & & & & & & $\hat{\vartheta} \approx$ & & 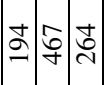 & 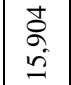 & $\mid$ & 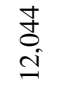 & 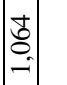 & 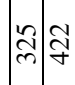 & $\cong$ & $\mid$ & 占 & 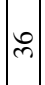 \\
\hline$\hat{\sigma}$ & $\$ \varsubsetneqq$ & & & & +0 & 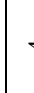 & t & & & & & & $\stackrel{\infty}{\infty} \mid \infty$ & & ఫీ & $\begin{array}{l}\mathfrak{b} \\
\stackrel{2}{a}\end{array}$ & 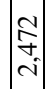 & $\begin{array}{l}\bar{\sigma} \\
\grave{\varrho}\end{array}$ & $\left|\begin{array}{l}0 \\
\infty \\
\infty\end{array}\right|$ & 省离 & $\nsubseteq$ & $\mid$\begin{tabular}{l|}
$\infty$ \\
$\infty$ \\
$\infty$
\end{tabular} & $\tilde{y}$ & $\approx$ \\
\hline$\delta$ & $\stackrel{\infty}{\circ}$ & & & & +0 & 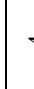 & t & & & & & & $\hat{n} \pm$ & & 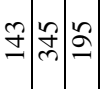 & 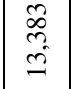 & $\mid \begin{array}{l}\vec{a} \\
\hat{i} \\
i\end{array}$ & लิ & 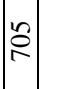 & ત્તిల్ల & $\stackrel{\circ}{\varrho}$ & $\stackrel{\circ}{\circ}$ & $\stackrel{8}{q}$ & 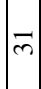 \\
\hline ลิ & $m த$ & & & & $\sim 0$ & 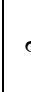 & v & & & & & & $\therefore$ & & 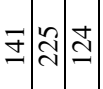 & $\mid$\begin{tabular}{l}
$\infty$ \\
$\stackrel{\infty}{0}$ \\
\multirow{=}{*}{}
\end{tabular} & | & $\begin{array}{l}\text { Oे } \\
\text { o. } \\
\infty\end{array}$ & d. & సิ & 8 & $\left|\begin{array}{c}\Delta \\
\infty \\
\infty\end{array}\right|$ & $\overline{\mathrm{i}}$ & i \\
\hline పิ & $=\approx$ & & & & -0 & 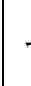 & 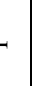 & & & & & & $=\backsim$ & & $2 \ln$ & $\mid \begin{array}{l}n \\
\stackrel{n}{c} \\
\underline{I}\end{array}$ & $\mid$ & $\begin{array}{l}\text { Oे } \\
\text { n. } \\
\infty\end{array}$ & $\mid \begin{array}{l}n \\
\infty \\
n\end{array}$ & ণ্ & $\stackrel{\Xi}{\circ}$ & 常 & $\stackrel{\sim}{\circ}$ & \\
\hline$\stackrel{\varrho}{\Xi}$ & 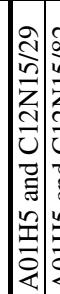 & & & & 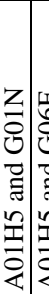 & & & 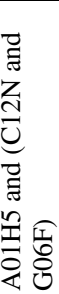 & 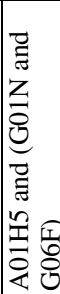 & 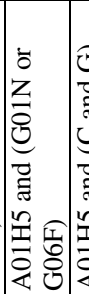 & 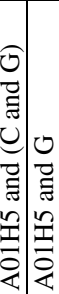 & & 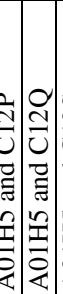 & 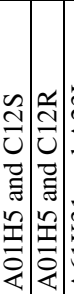 & 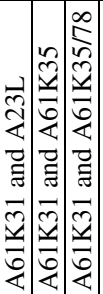 & 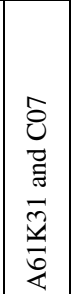 & $\begin{array}{l}0 \\
0 \\
0 \\
0 \\
0 \\
\vdots \\
\vdots \\
0 \\
0 \\
0 \\
0 \\
\end{array}$ & 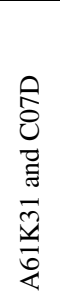 & 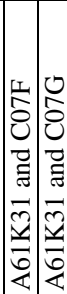 & 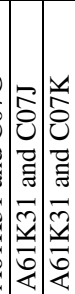 & 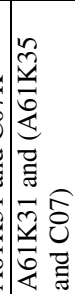 & 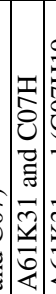 & 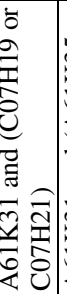 & 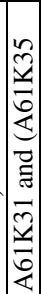 \\
\hline
\end{tabular}




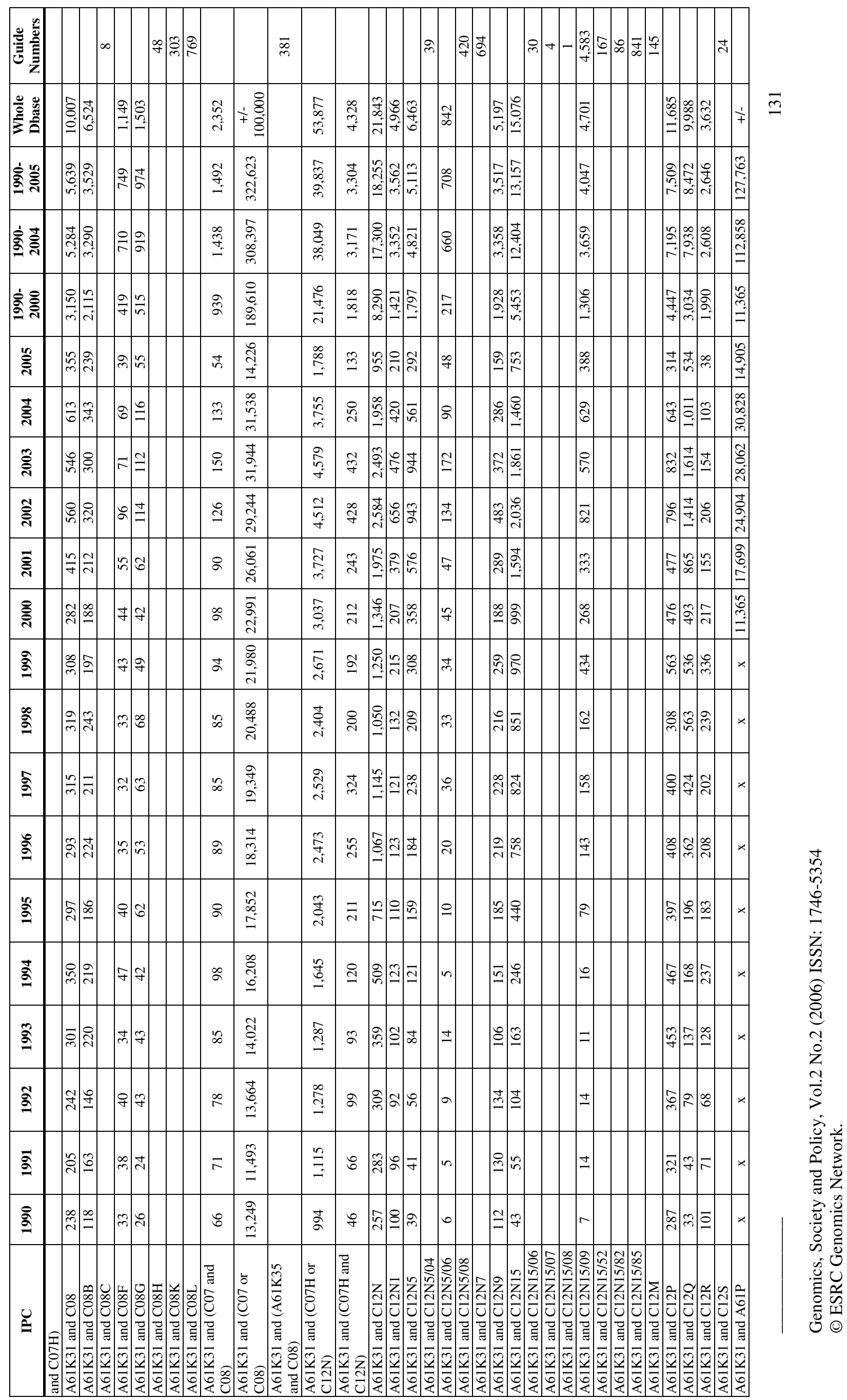


III

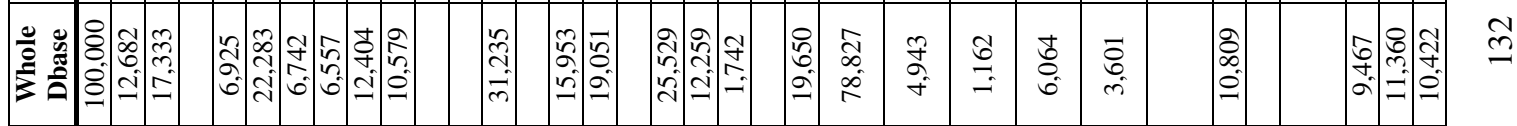

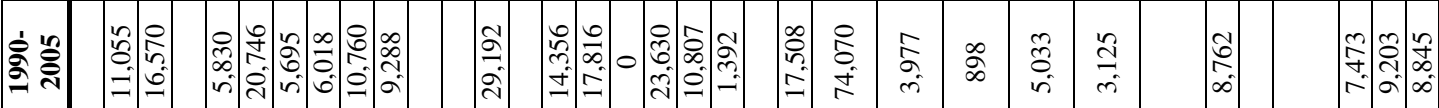

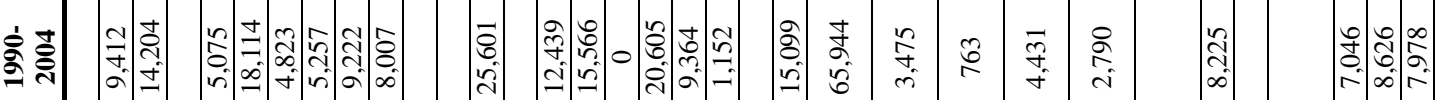

言离

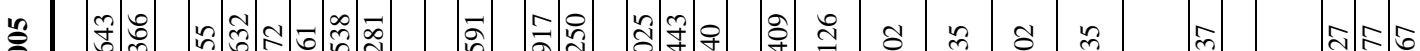

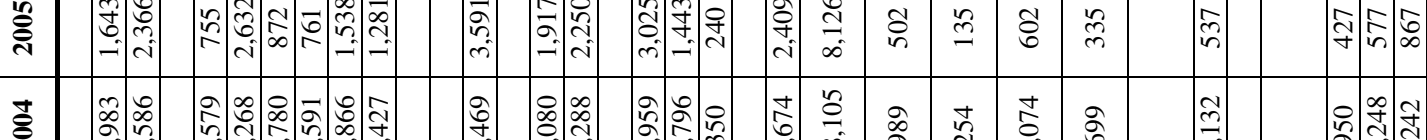

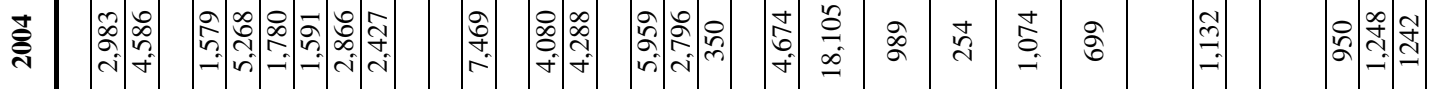

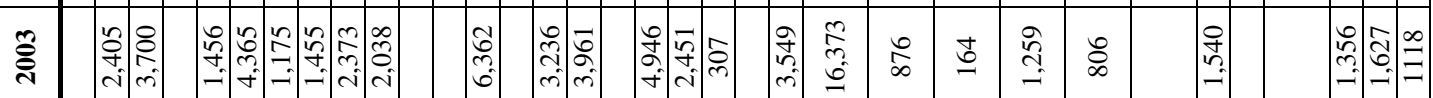

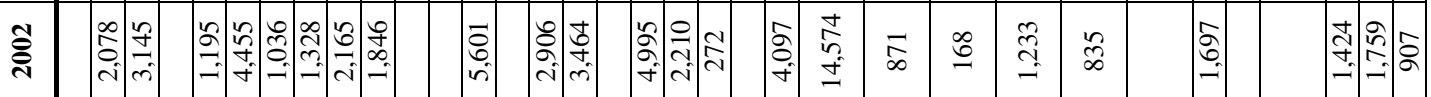

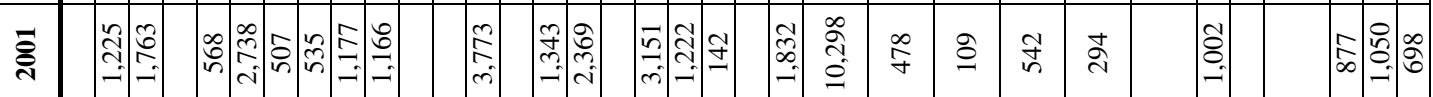

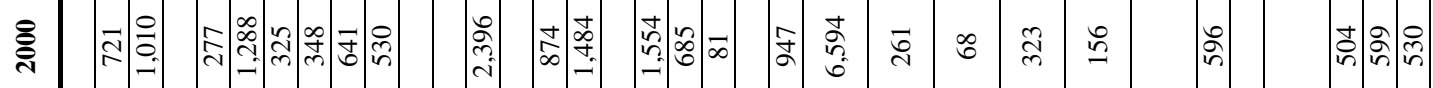

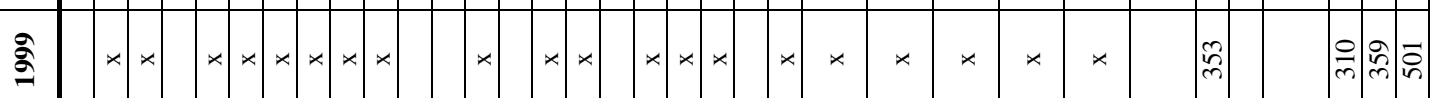

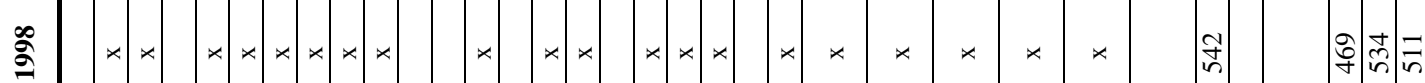

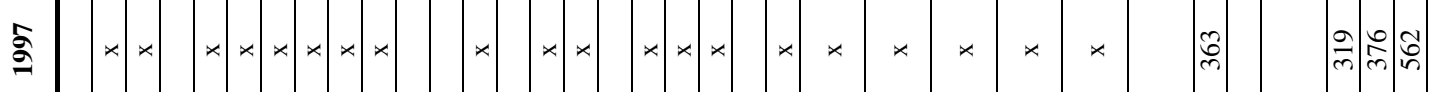

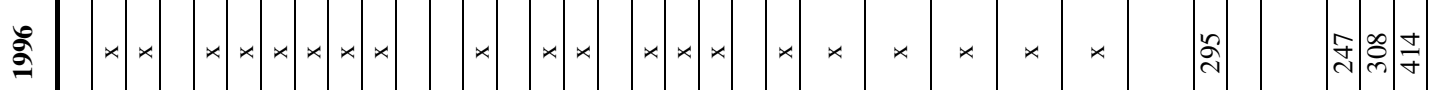

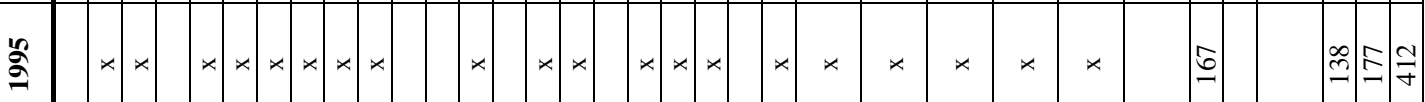

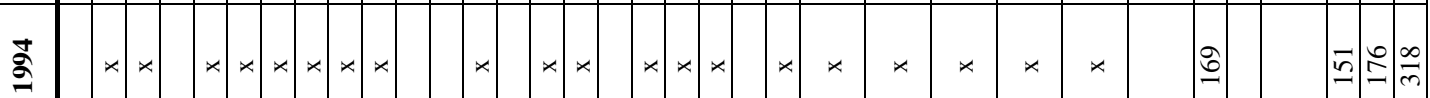

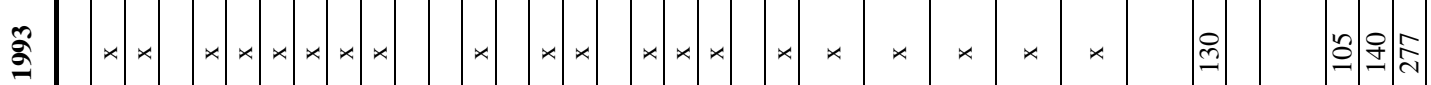

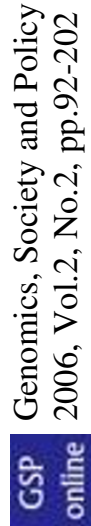

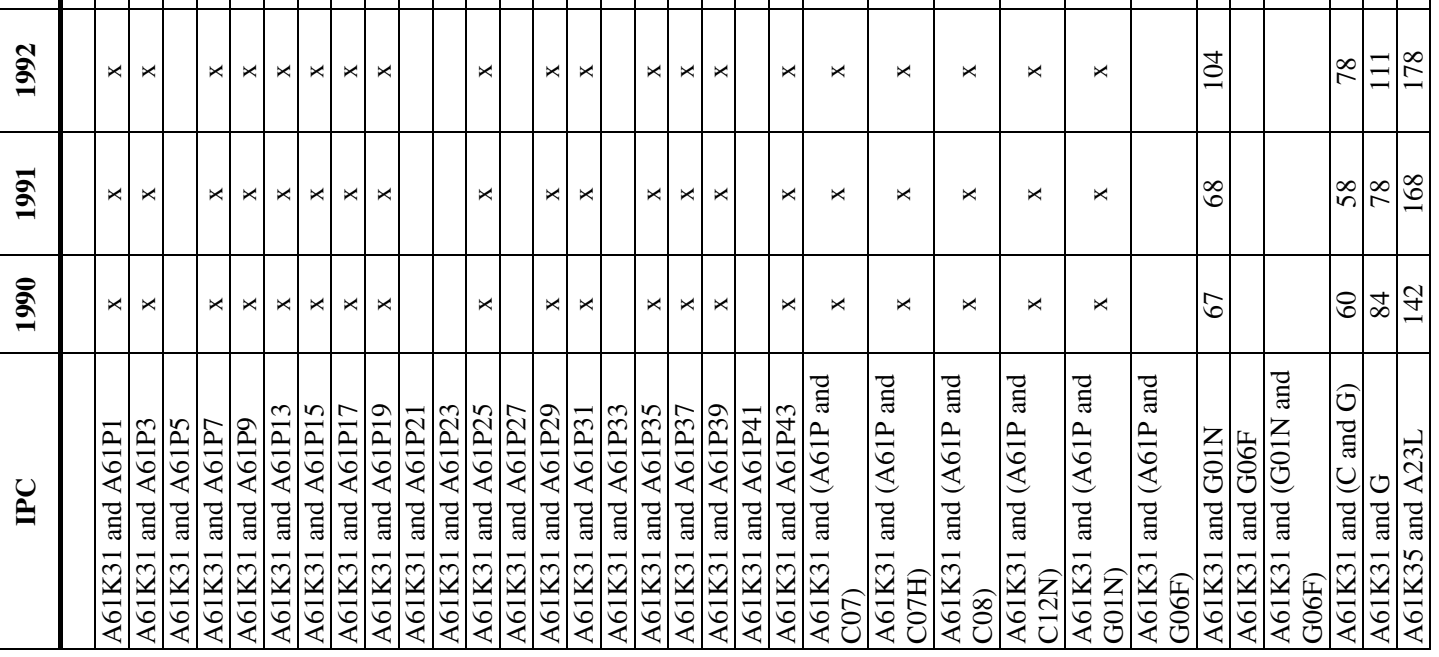




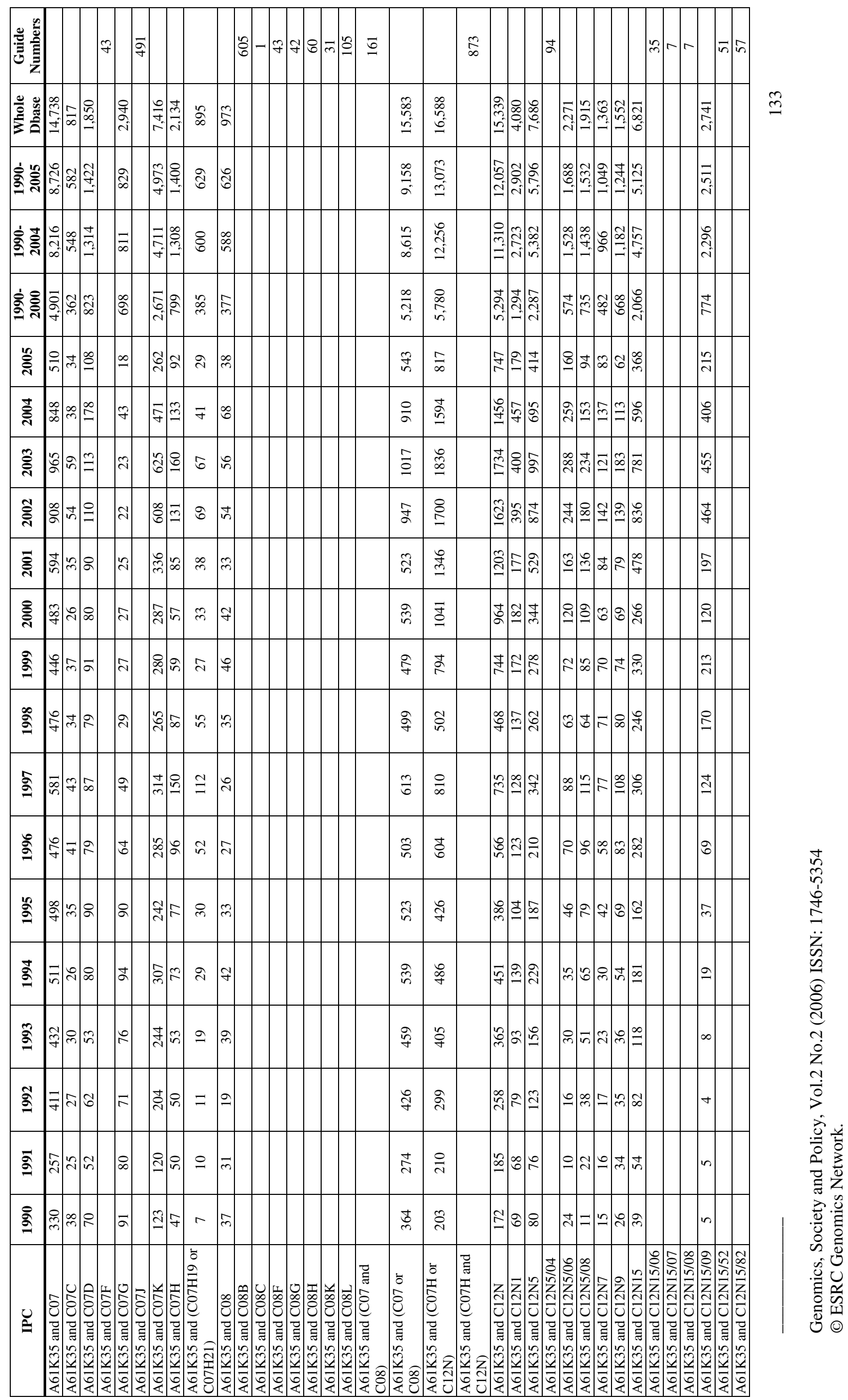




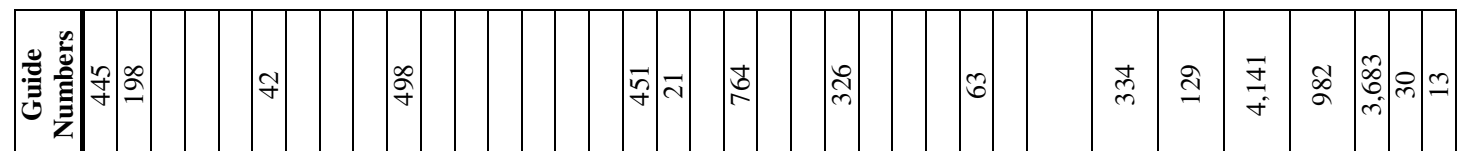

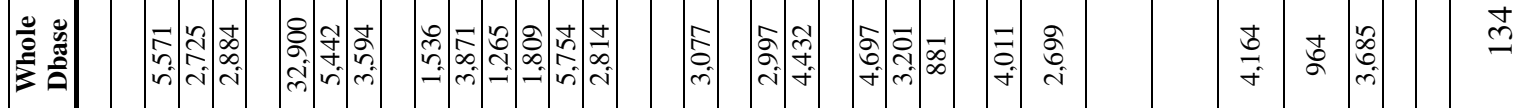

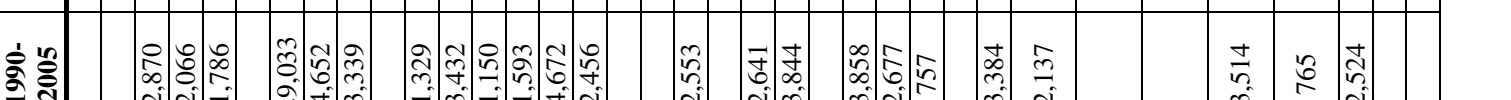

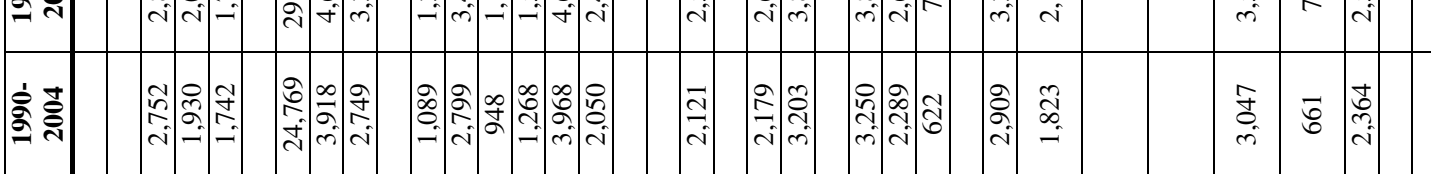

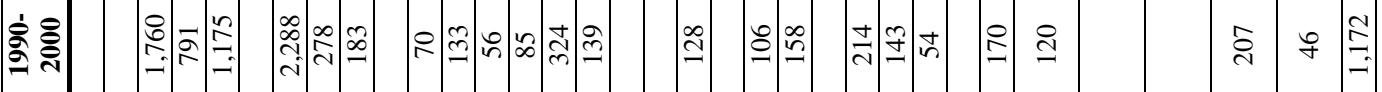

竎

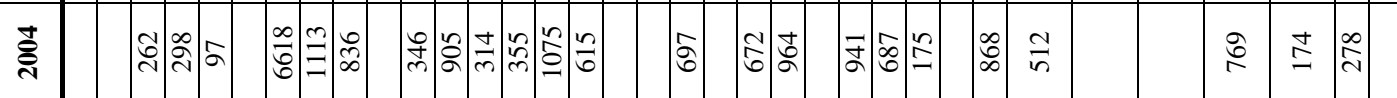

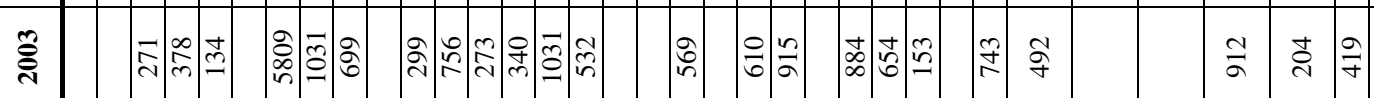

帘

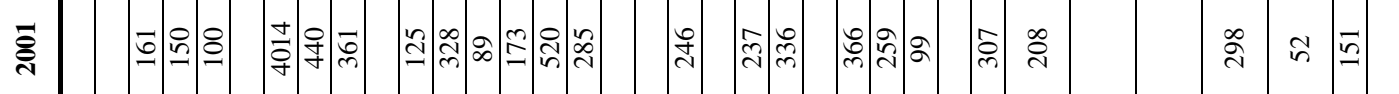

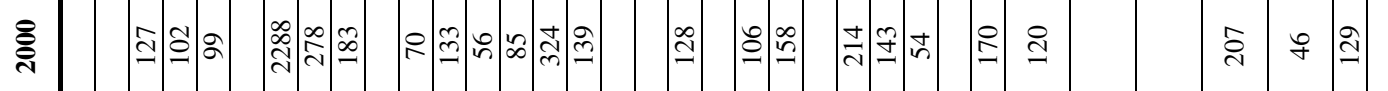

ลิ

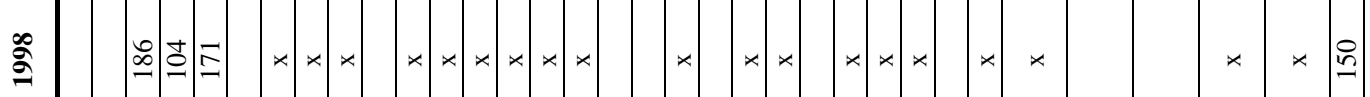

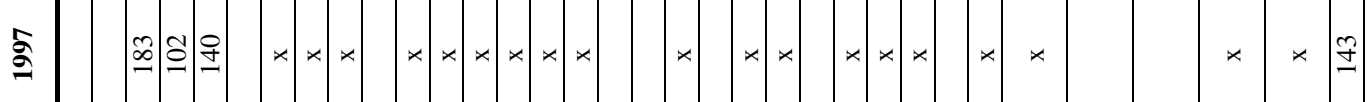

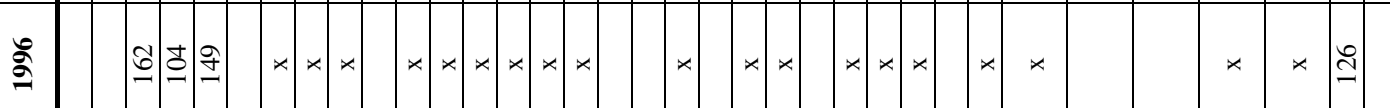

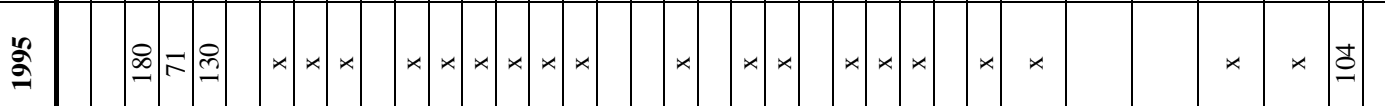

2 ㅎำ

2

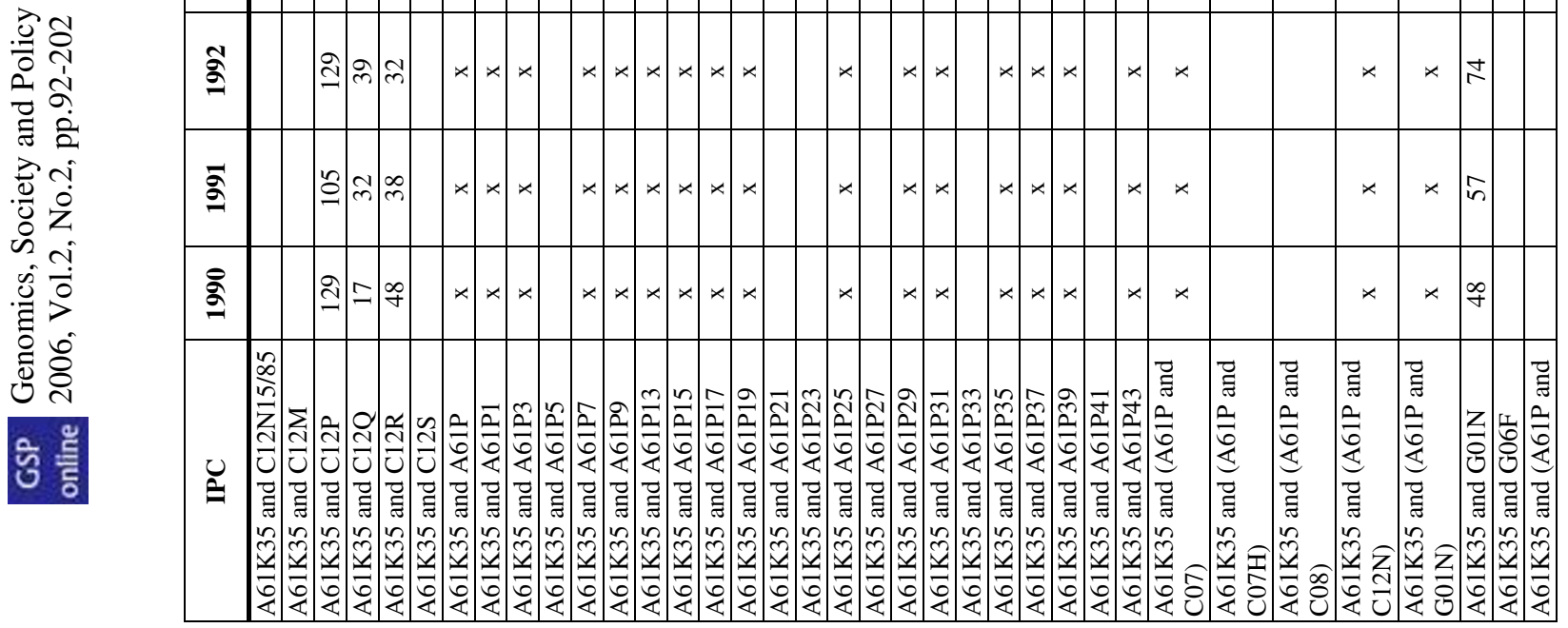

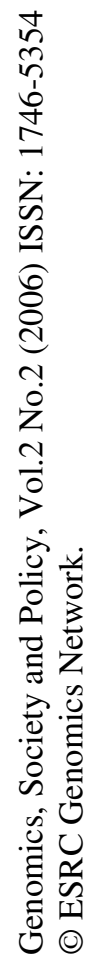




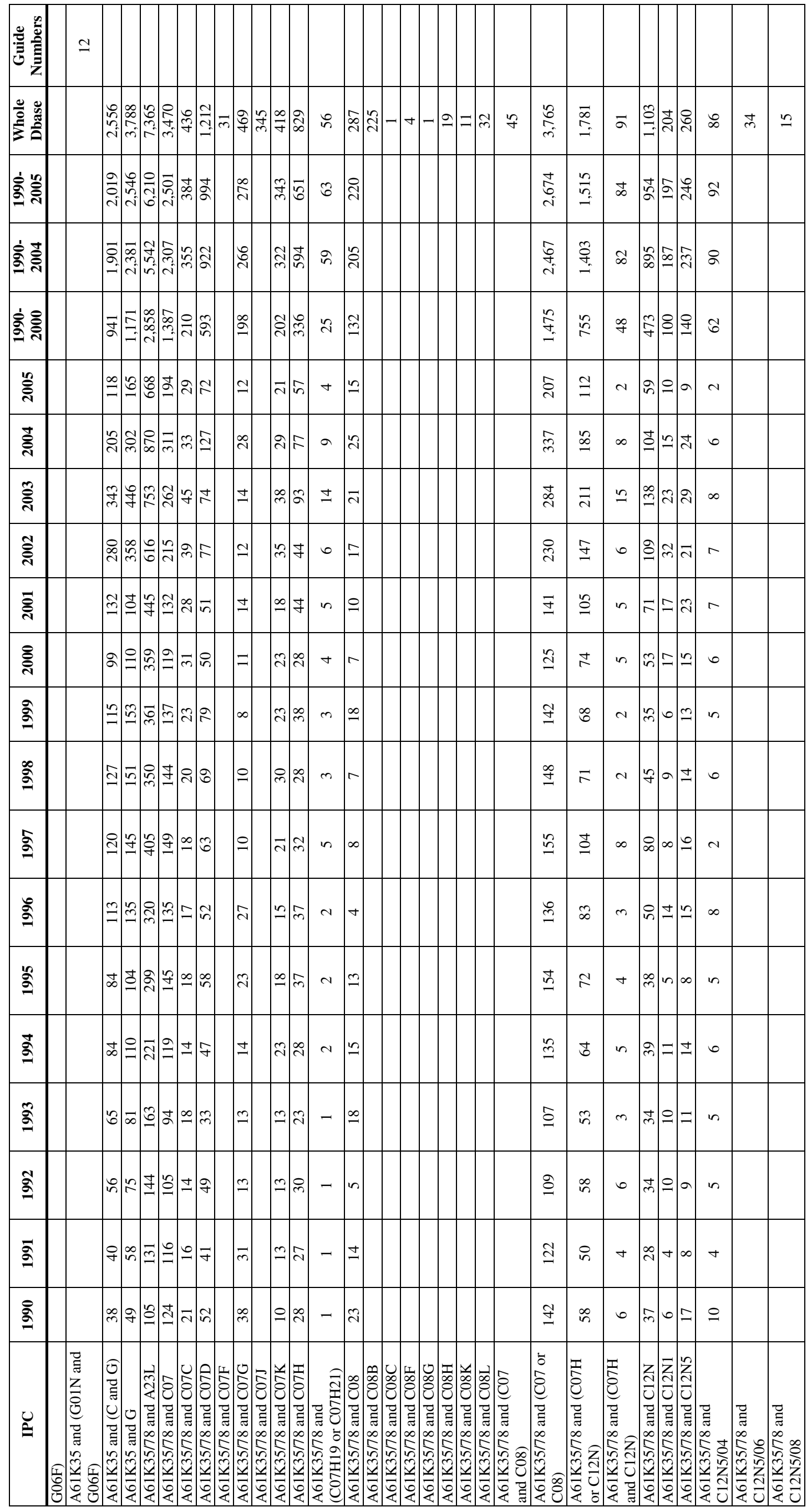




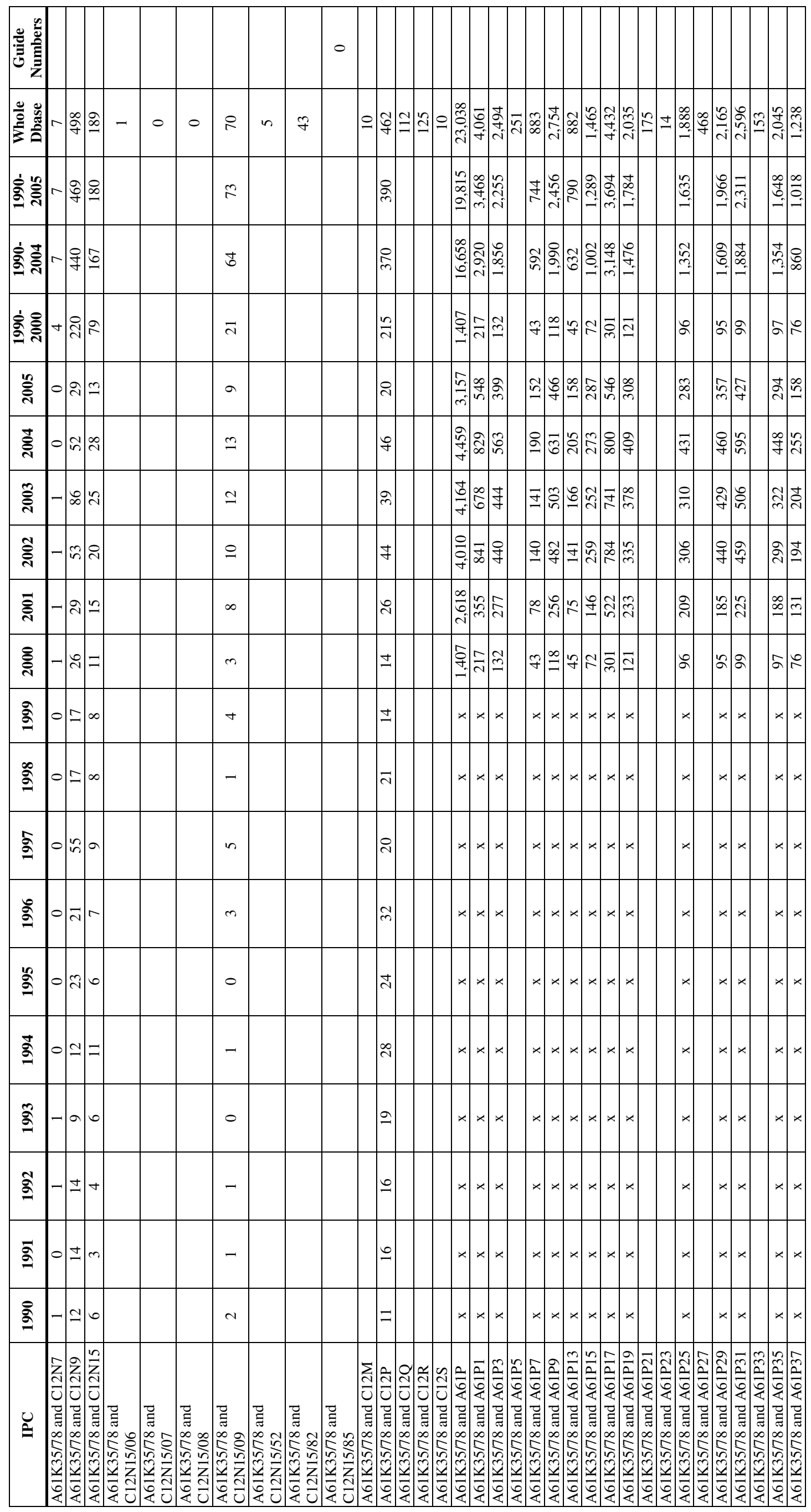




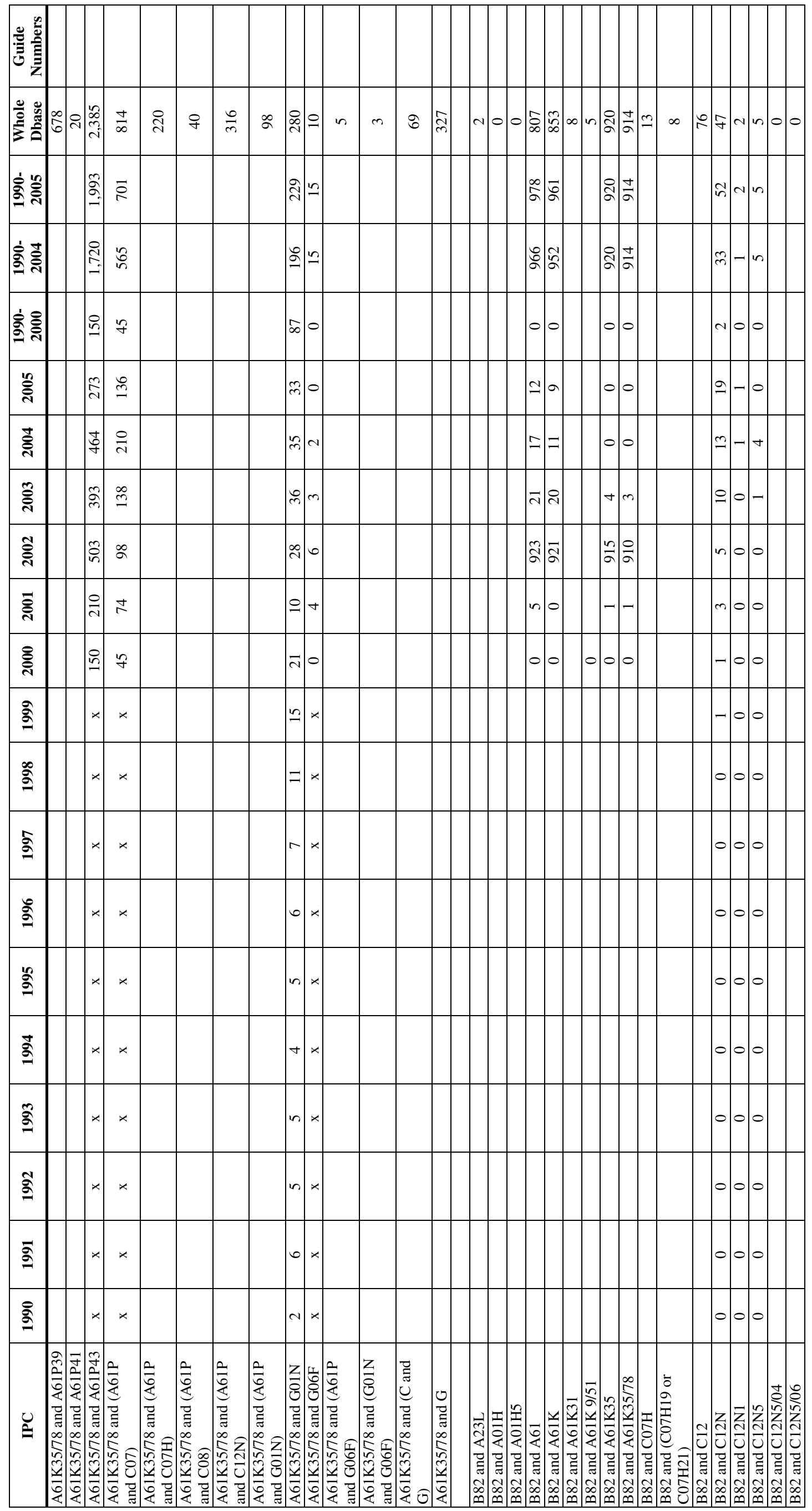




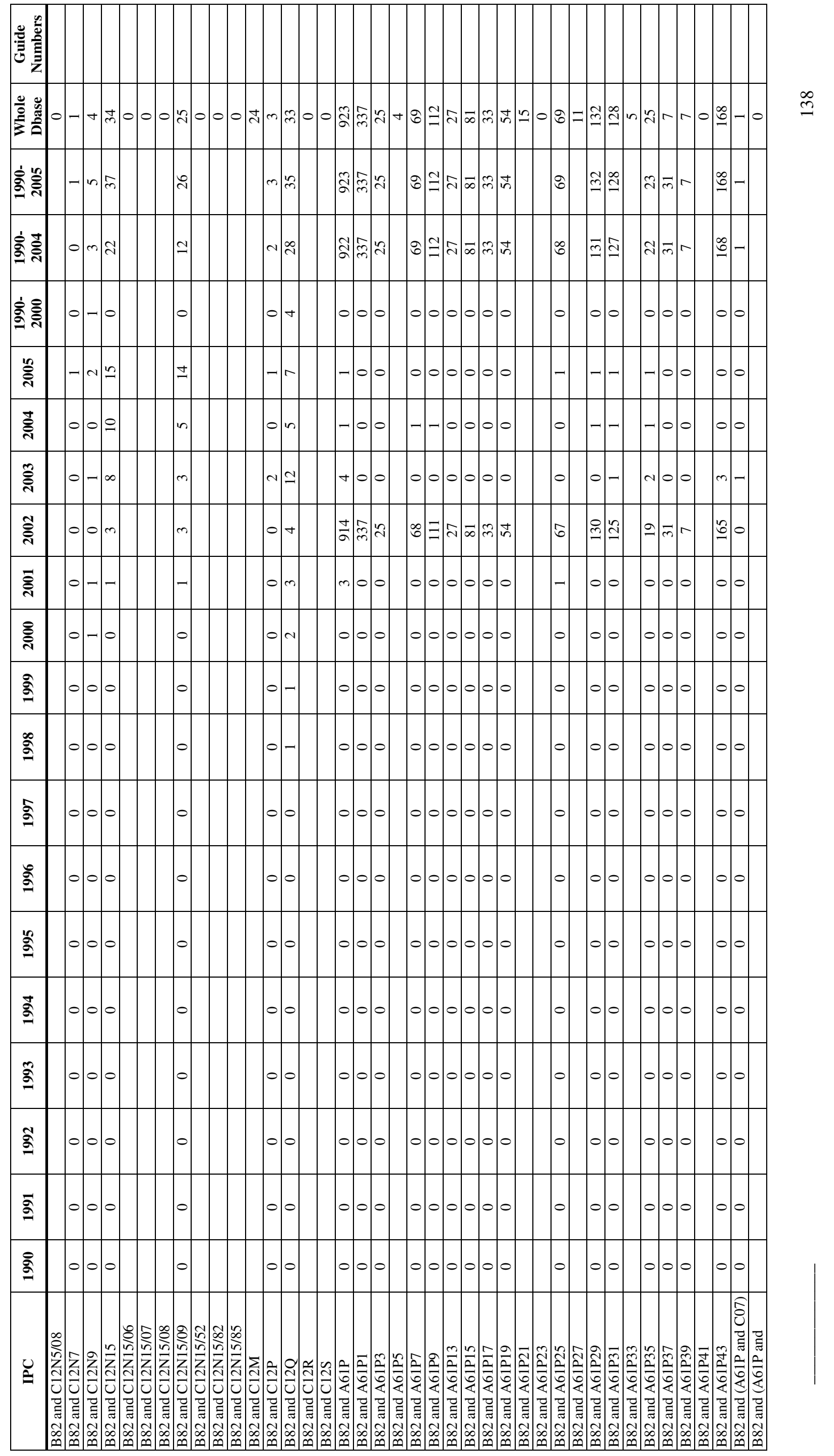




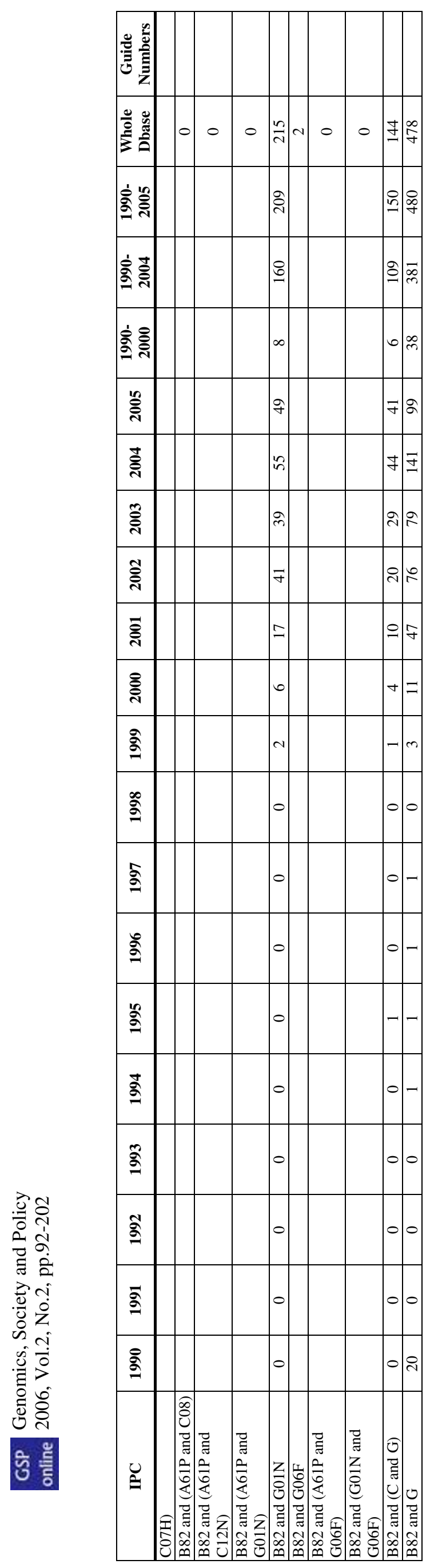




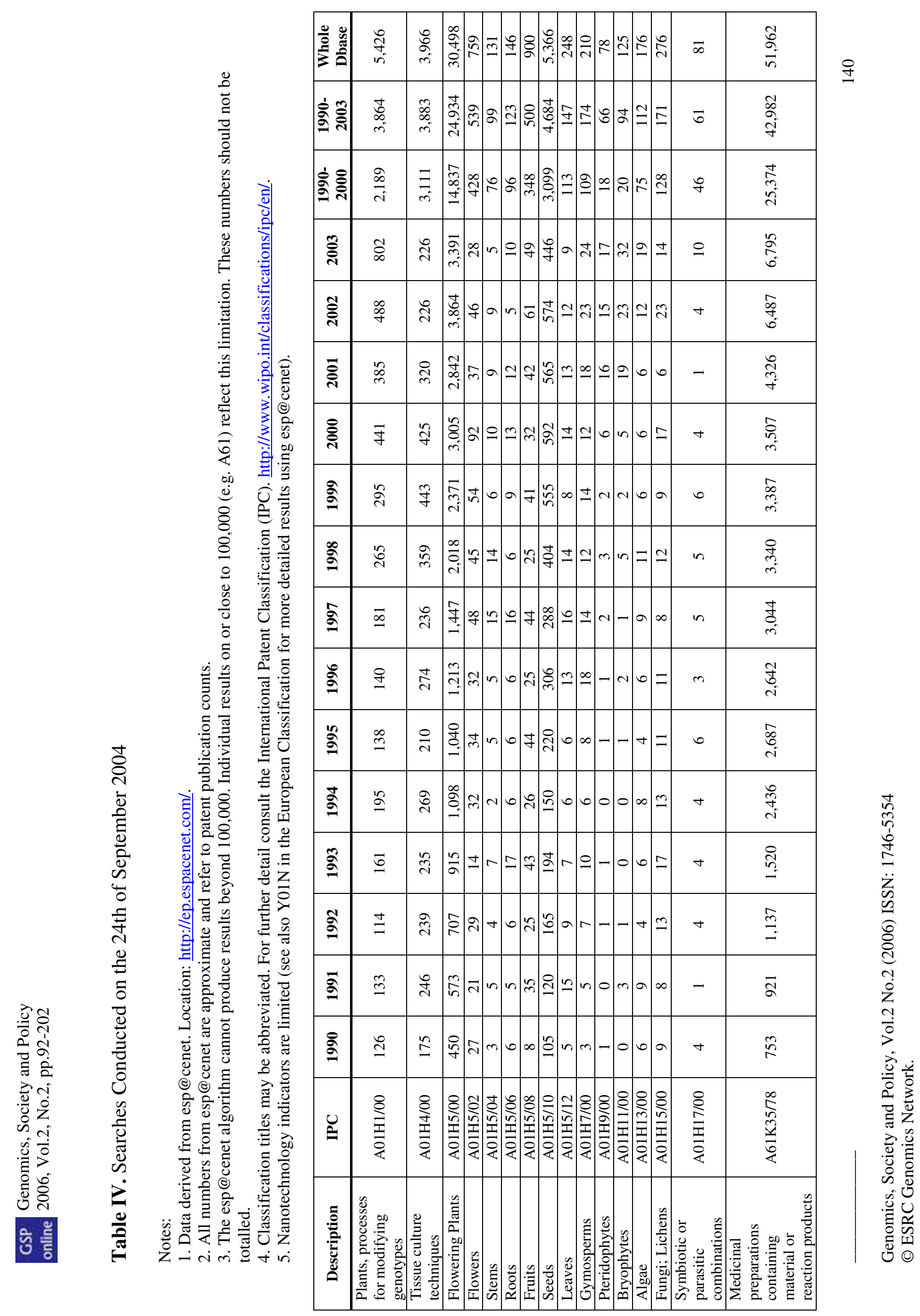




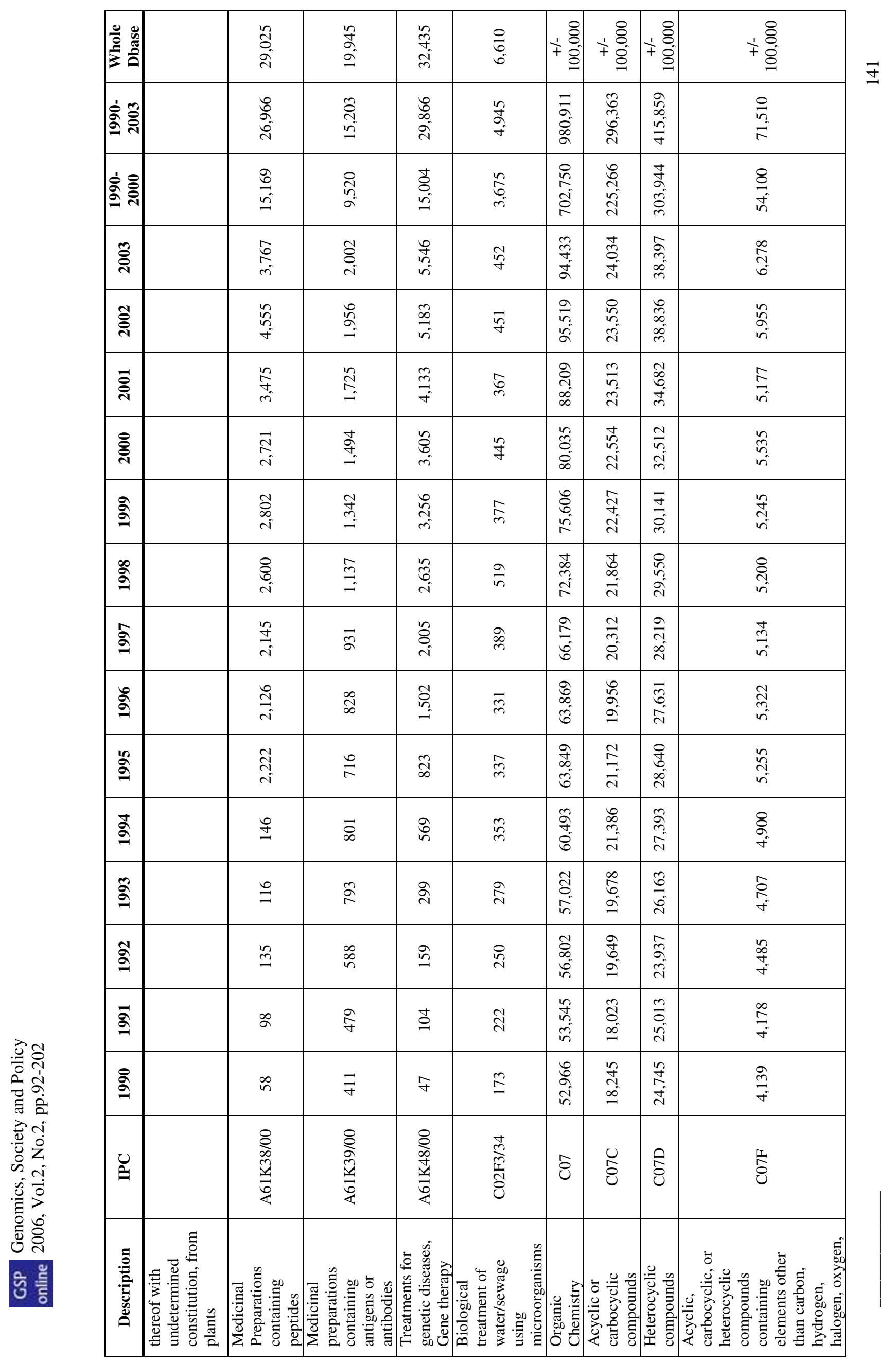




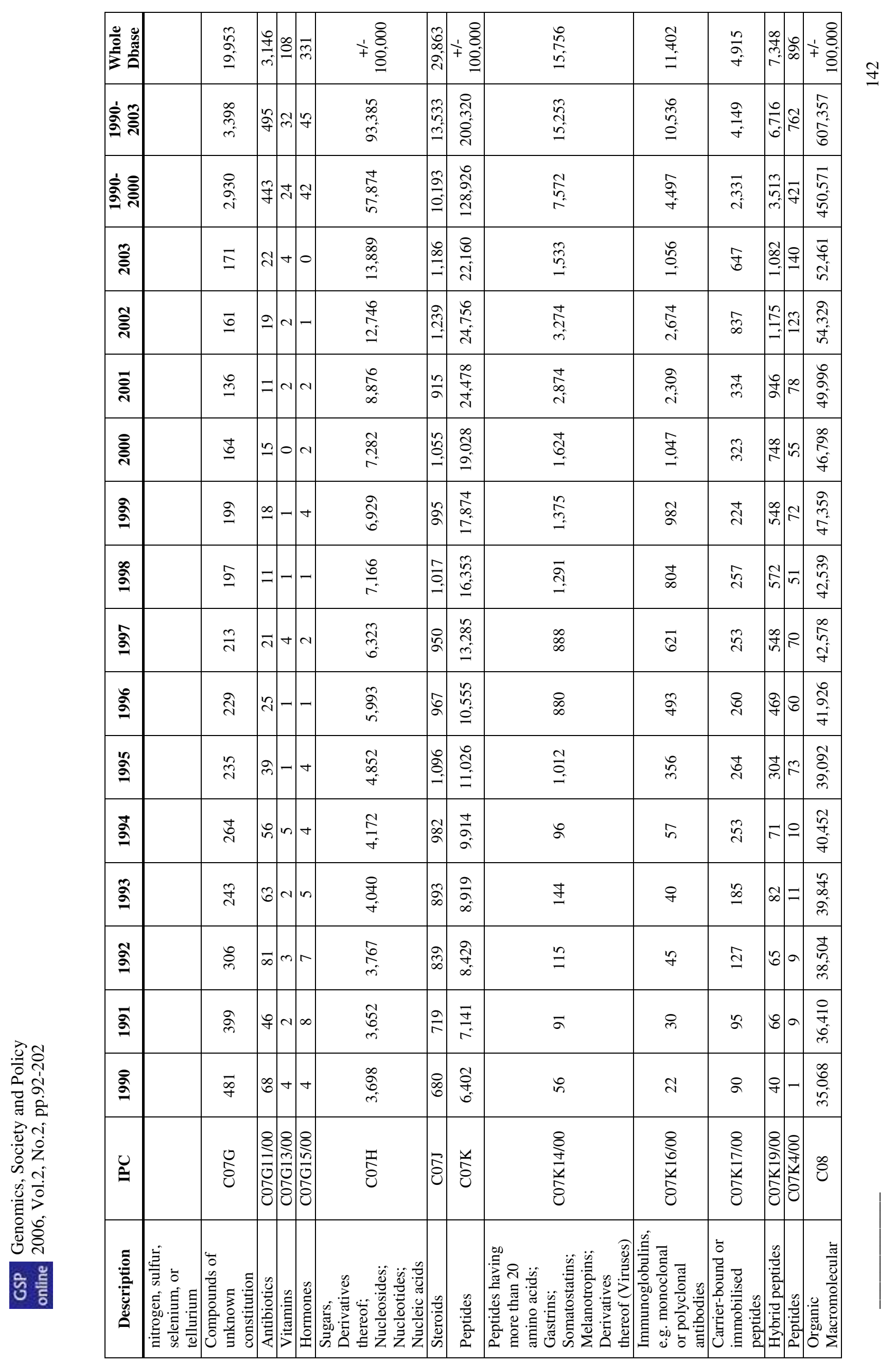

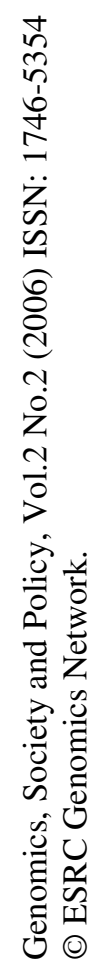




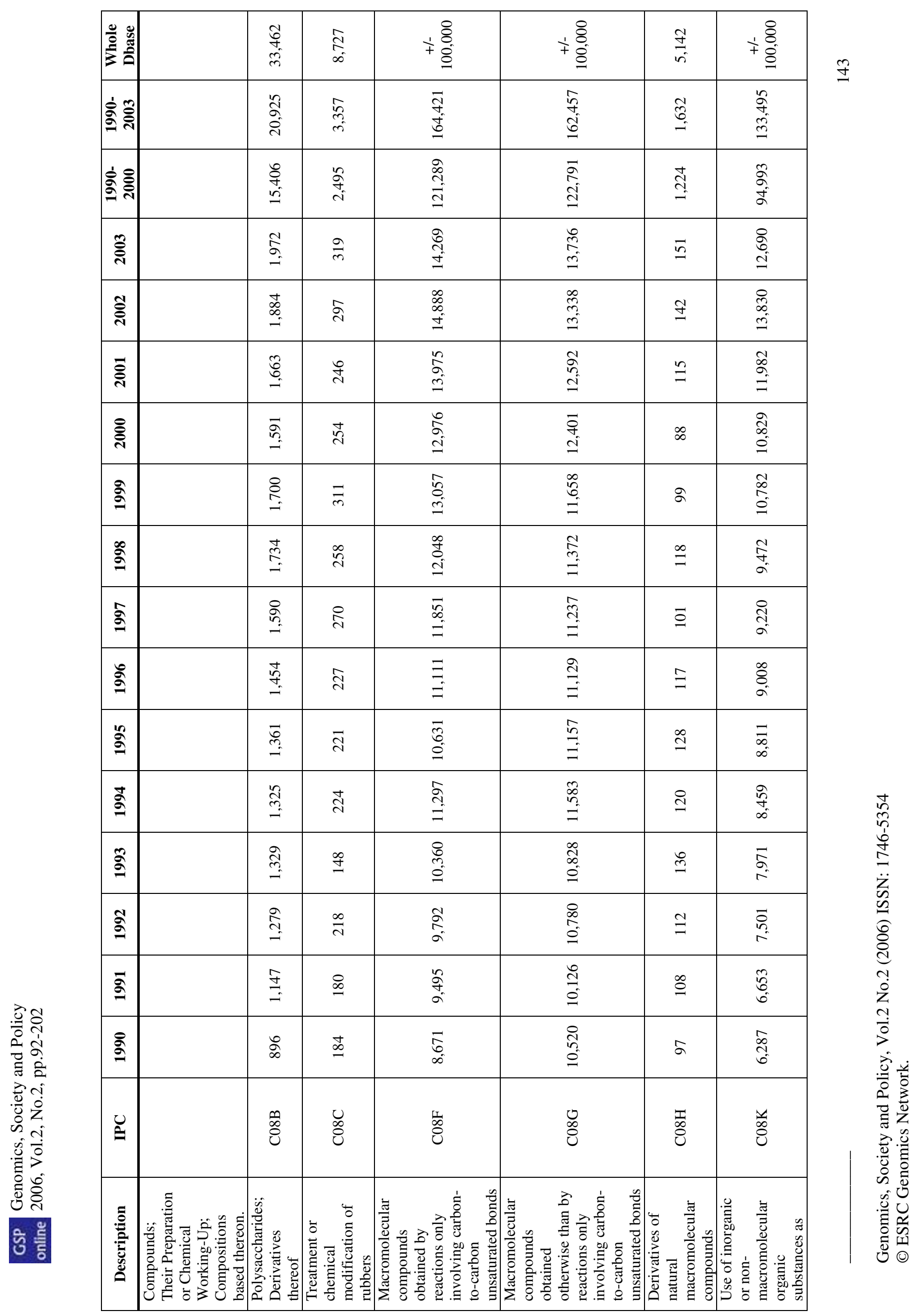




\begin{tabular}{|c|c|c|c|c|c|c|c|c|c|c|}
\hline$\frac{2}{2}$ & 它兽 & 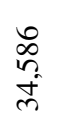 & 字兽 & $\frac{b}{n}$ & & 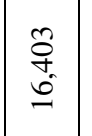 & 亳 & 苞 & 它密 & $\stackrel{\mathrm{m}}{\mathrm{m}}$ \\
\hline 譬离 & 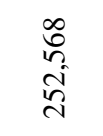 & $\begin{array}{l}\text { त̂ } \\
\text { dy }\end{array}$ & 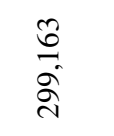 & : & $\begin{array}{l}: \\
\stackrel{0}{+}\end{array}$ & 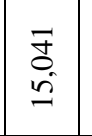 & ڤ్ & $\begin{array}{l}\stackrel{r}{\infty} \\
\stackrel{\infty}{=} \\
=\end{array}$ & 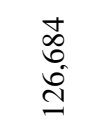 & $\frac{b}{m}$ \\
\hline 䜌 & 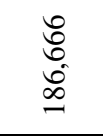 & 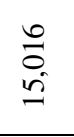 & 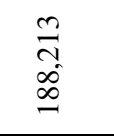 & 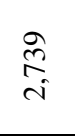 & 商 & 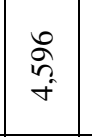 & 总 & 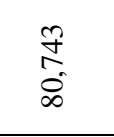 & $\begin{array}{l}\text { \% } \\
\text { i. } \\
\text { in }\end{array}$ & 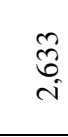 \\
\hline 总 & $\stackrel{\infty}{=}$ & च̃ & 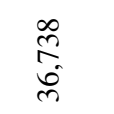 & $\underset{\infty}{\vec{\infty}}$ & $\vec{\infty}$ & \begin{tabular}{|c|} 
\\
$\infty$ \\
$\infty$ \\
$i$
\end{tabular} & $\overrightarrow{\mathrm{d}}$ & 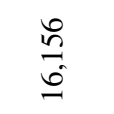 & $\begin{array}{l}\text { 学 } \\
\text { a }\end{array}$ & $\stackrel{\sim}{\circ}$ \\
\hline స్ & 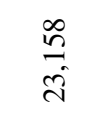 & 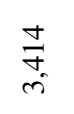 & $\begin{array}{l}\stackrel{2}{2} \\
\text { d. }\end{array}$ & 售 & 点 & \begin{tabular}{|l|} 
\\
$\tilde{n}$ \\
$\tilde{m}$
\end{tabular} & $\stackrel{\substack{0 \\
:}}{-i}$ & $\frac{\infty}{\underline{m}}$ & 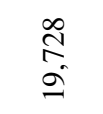 & $\bar{\Xi}$ \\
\hline స్ & $\begin{array}{l}0 \\
\stackrel{0}{0} \\
\text { i }\end{array}$ & $\begin{array}{l}\vec{\pi} \\
\vec{n}\end{array}$ & $\begin{array}{l}\text { 今े } \\
\text { is }\end{array}$ & $\stackrel{\circ}{\circ}$ & $\stackrel{\circ}{i n}$ & 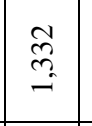 & 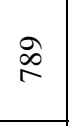 & $\begin{array}{l}\text { 离 } \\
\infty \\
\infty\end{array}$ & 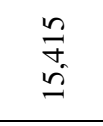 & $\cong$ \\
\hline 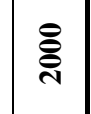 & $\begin{array}{l}\overrightarrow{\vec{a}} \\
\stackrel{a}{\Omega}\end{array}$ & 晜 & 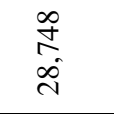 & $\frac{\infty}{n}$ & $\frac{\infty}{n}$ & $\begin{array}{c}\mathscr{n} \\
\infty \\
\infty\end{array}$ & 雍 & 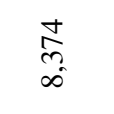 & $\begin{array}{l}\underset{F}{\vec{f}} \\
\text { I }\end{array}$ & $\stackrel{8}{\circ}$ \\
\hline बे & $\begin{array}{l}\hat{n} \\
\hat{\infty} \\
\underline{c}\end{array}$ & 尔 & $\begin{array}{l}\infty \\
0 \\
\infty \\
0 \\
\text { ch }\end{array}$ & $\frac{\circ}{m}$ & $\stackrel{\circ}{i n}$ & $\hat{0}$ & i & $\begin{array}{l}\text { 学 } \\
\infty\end{array}$ & $\begin{array}{l}\text { 至 } \\
\text { s. }\end{array}$ & త్తి \\
\hline$\stackrel{\infty}{\stackrel{\infty}{2}}$ & 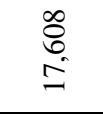 & 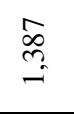 & $\begin{array}{l}\text { co } \\
\text { à } \\
\text { a }\end{array}$ & ন্ల & $\overbrace{}^{2}$ & $\frac{n}{6}$ & $\vec{b}$ & $\begin{array}{l}\infty \\
\substack{\infty \\
\infty} \\
\end{array}$ & 莕 & 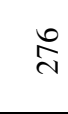 \\
\hline$\stackrel{\hat{\sigma}}{\underline{a}}$ & $\begin{array}{ll}n \\
c \\
c \\
n \\
n\end{array}$ & స్త్ & $\begin{array}{l}\tilde{y} \\
\stackrel{f}{\infty}\end{array}$ & 亏े & 亏े & $\frac{\infty}{n}$ & 等 & 雾 & 毶 & $\stackrel{\grave{c}}{ }$ \\
\hline$\stackrel{\circ}{\circ}$ & 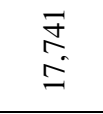 & 卷 & 先 & $\tilde{\pi}$ & $\tilde{a}$ & $\mathscr{g}$ & $\stackrel{\infty}{\infty}$ & 윳 & 总 & ลิ \\
\hline$\stackrel{0}{\stackrel{0}{g}}$ & $\begin{array}{l}\stackrel{\pi}{0} \\
\stackrel{0}{0}\end{array}$ & $\stackrel{\circ}{\frac{0}{3}}$ & 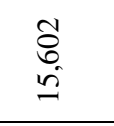 & $\Xi$ & $\Xi$ & F & $\stackrel{m}{m}$ & $\stackrel{R}{R}$ & 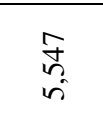 & f \\
\hline 萦 & $\begin{array}{l}0 \\
0 \\
0 \\
0 \\
0\end{array}$ & $\stackrel{\vec{g}}{=}$ & $\begin{array}{l}\mathscr{\infty} \\
\underset{f}{q}\end{array}$ & $\stackrel{8}{\circ}$ & $\stackrel{8}{\circ}$ & 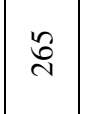 & בे & 命 & 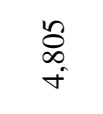 & 志 \\
\hline$\stackrel{0}{g}$ & $\begin{array}{l}\text { 茴: } \\
\stackrel{0}{0}\end{array}$ & 点 & 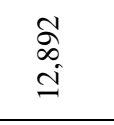 & $\stackrel{\circ}{\circ}$ & $\stackrel{8}{-1}$ & $\ddot{n}$ & 守 & 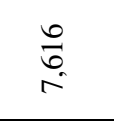 & 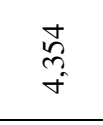 & đ̇ \\
\hline ఏ̃ & 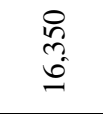 & 离 & 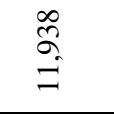 & 龺 & 孛 & $\stackrel{\circ}{\sim}$ & $\Xi$ & : & 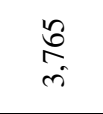 & : \\
\hline $\bar{\Xi}$ & $\begin{array}{l}\infty \\
\text { o } \\
+ \\
+\end{array}$ & $\stackrel{ \pm}{ \pm}$ & $\begin{array}{l}\text { 总 } \\
\stackrel{0}{0.0}\end{array}$ & $\stackrel{\circ}{\dddot{2}}$ & $\stackrel{\circ}{\sim}$ & $\begin{array}{l} \pm \\
\stackrel{ \pm}{*}\end{array}$ & $\Xi$ & $\begin{array}{l}\text { तु } \\
\text { वेt }\end{array}$ & 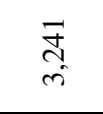 & $\stackrel{+}{g}$ \\
\hline$\stackrel{2}{2}$ & $\begin{array}{l}f \\
\text { 柔 }\end{array}$ & 芼 & 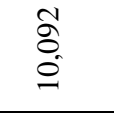 & $\stackrel{g}{\dddot{m}}$ & $\stackrel{\circ}{\rightarrow}$ & $\stackrel{\mathrm{d}}{.}$ & 8 & $\frac{9}{\bar{\sigma}}$ & 㞼 & \& \\
\hline 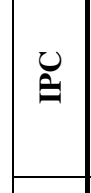 & 总 & 胥 & 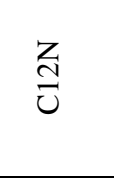 & $\begin{array}{l}\text { 壱 } \\
\text { d } \\
\text { d }\end{array}$ & 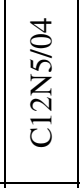 & 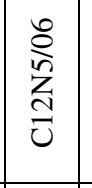 & 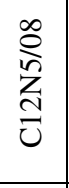 & ปี & $\frac{g}{\tilde{U}}$ & $\stackrel{\mathscr{U}}{\tilde{U}}$ \\
\hline 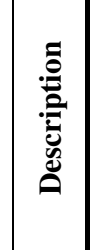 & & & & & & & & & & \\
\hline
\end{tabular}




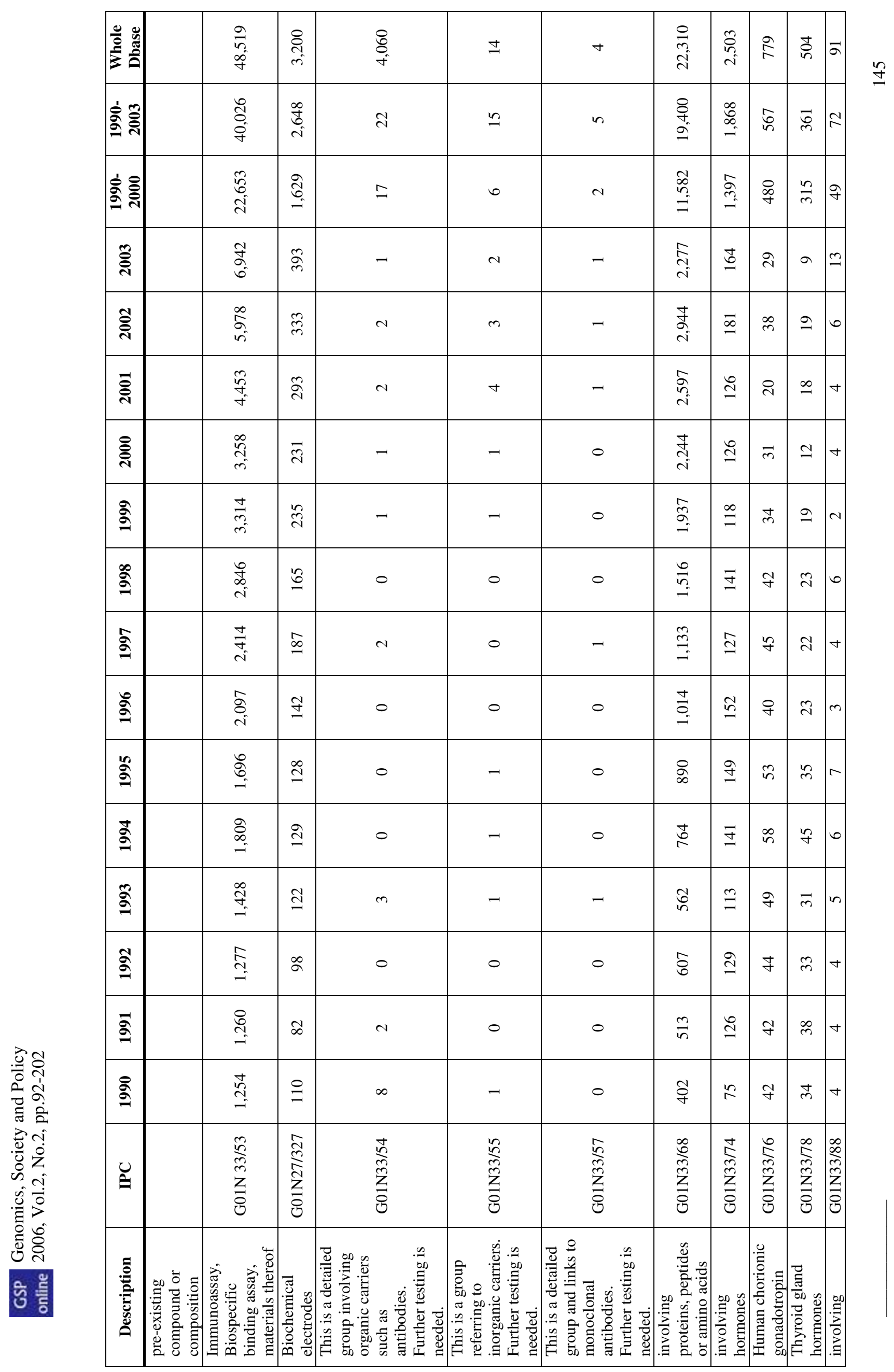




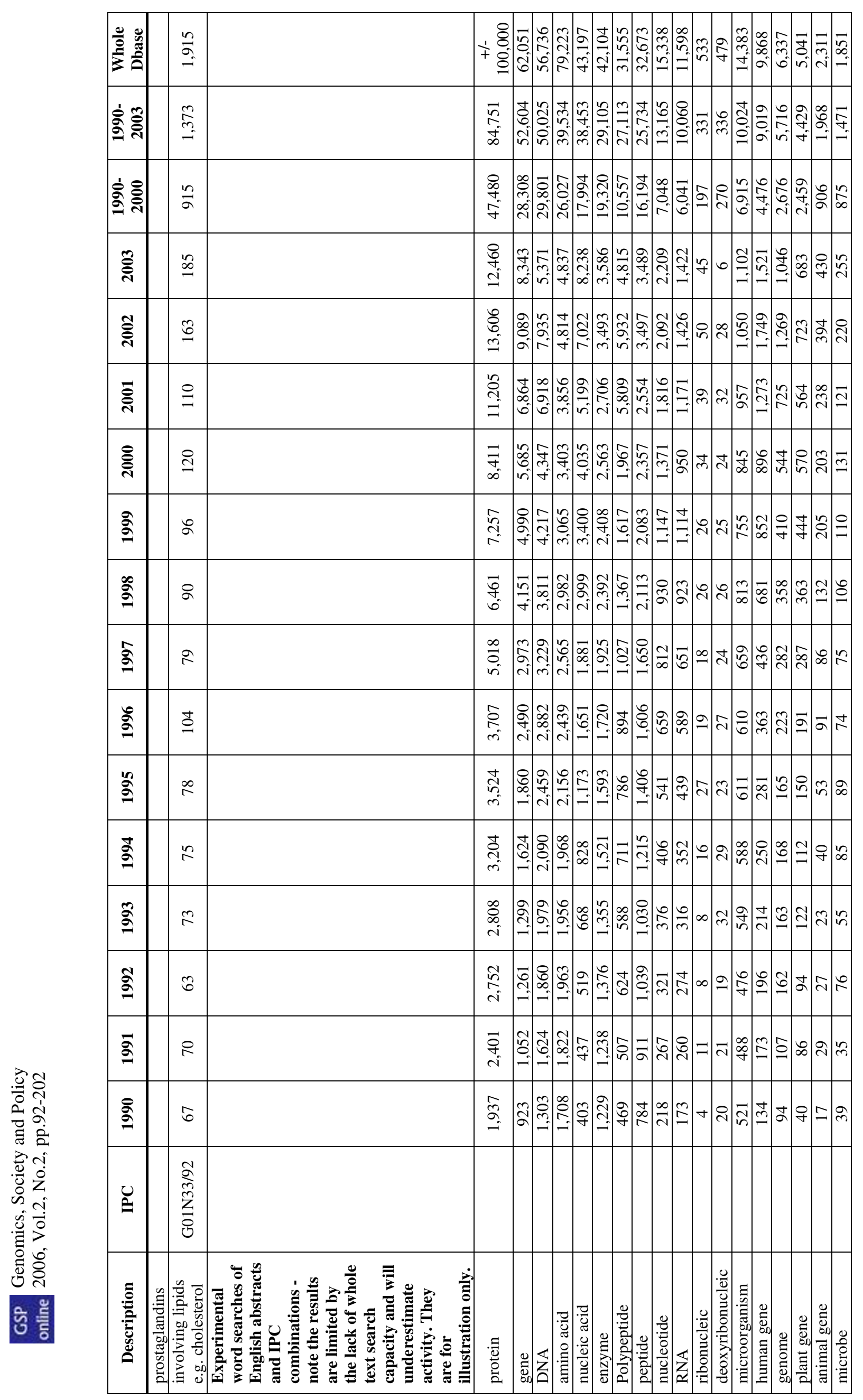




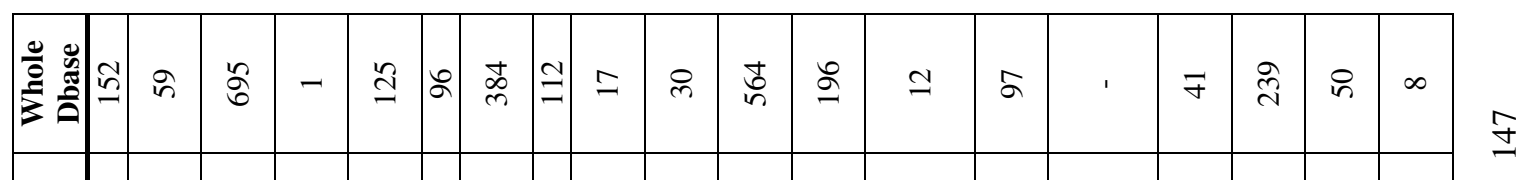

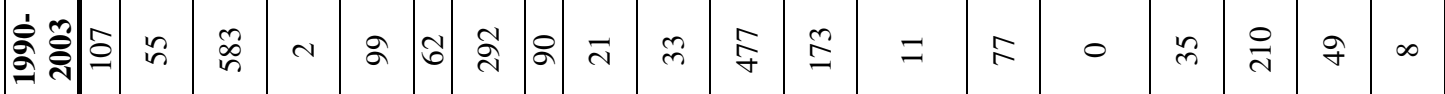
ลิ่ 气ิิ 气ิે ฮิ่

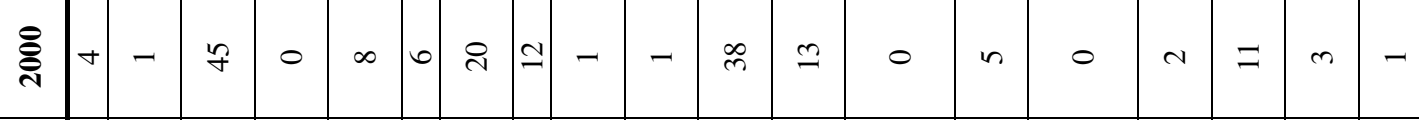

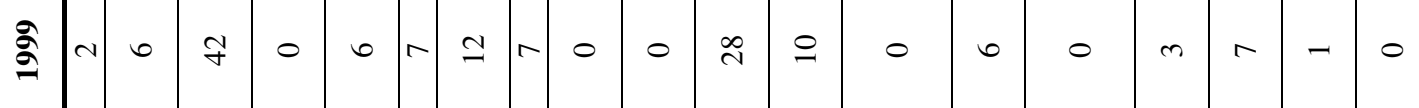

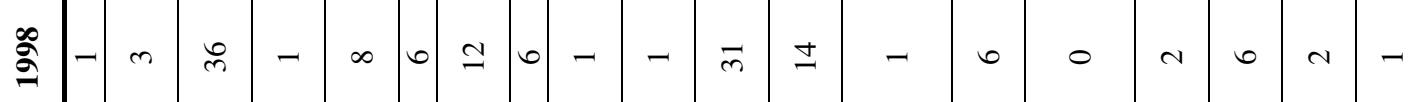
$\hat{g}$

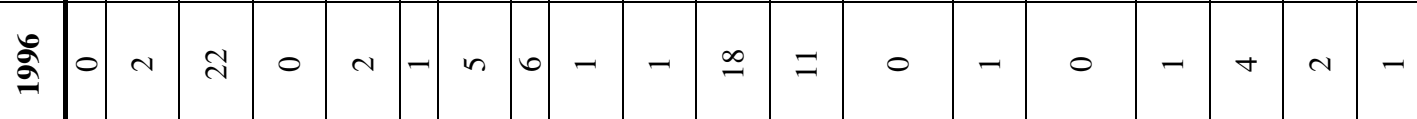
$\stackrel{\circ}{\circ}$

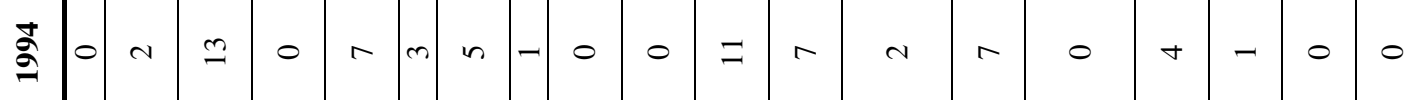

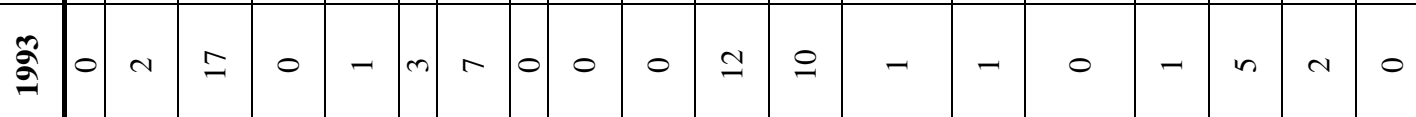

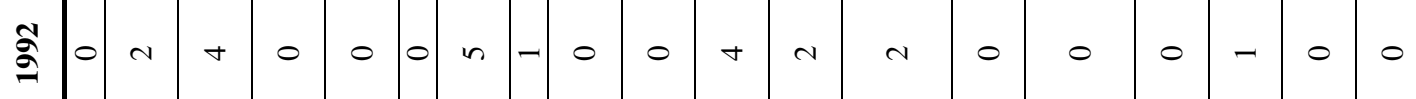

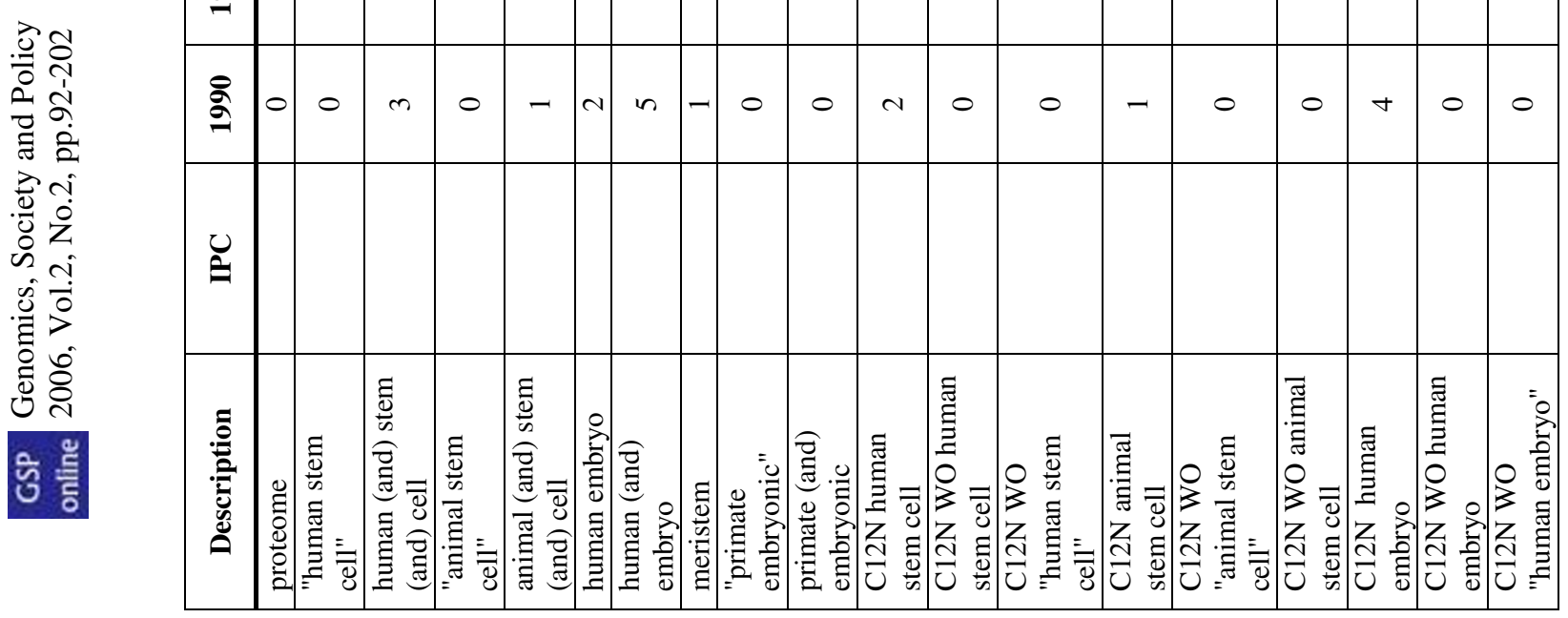

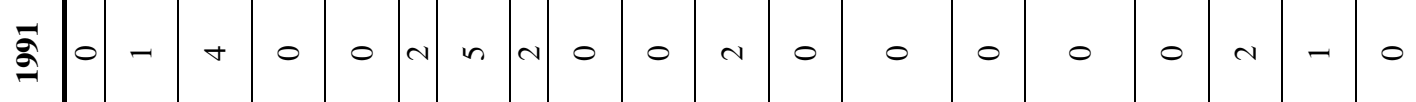




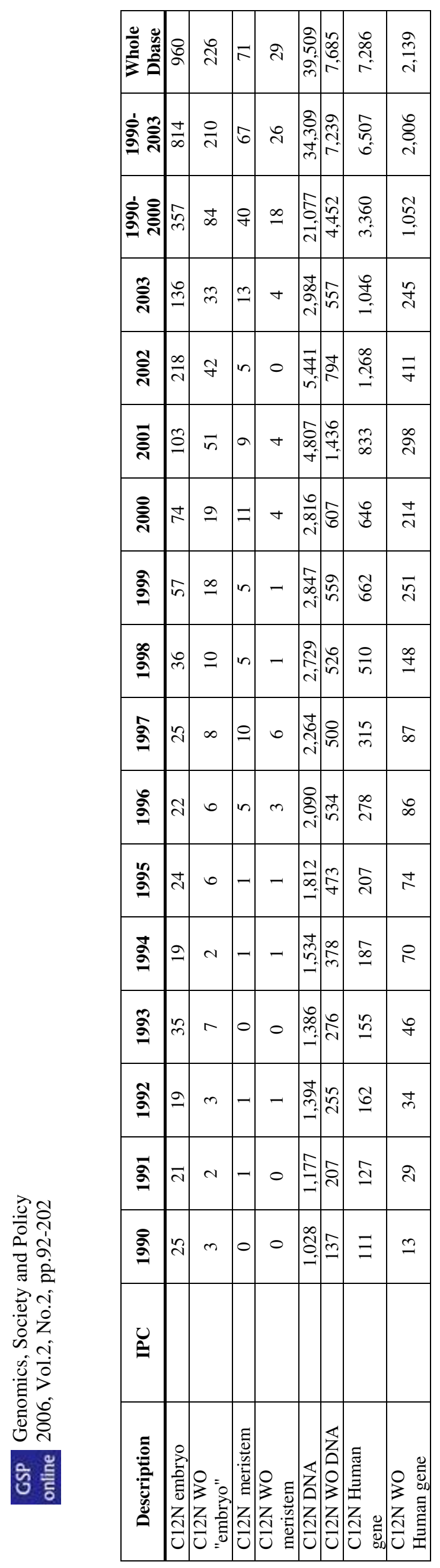




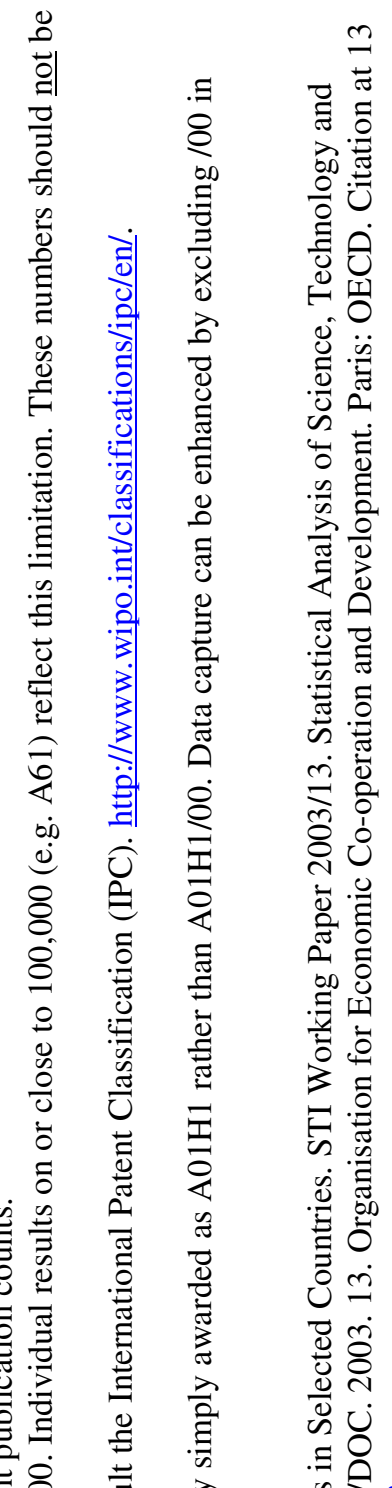

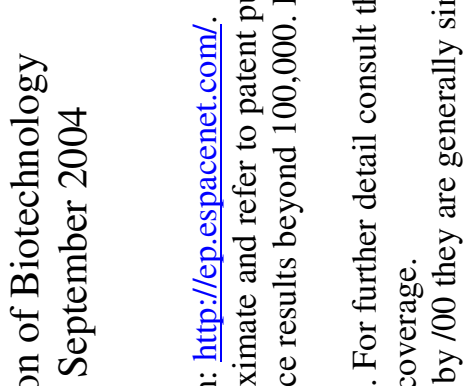

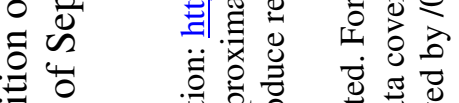

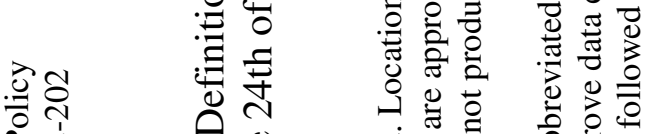

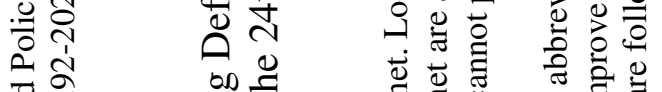

它全

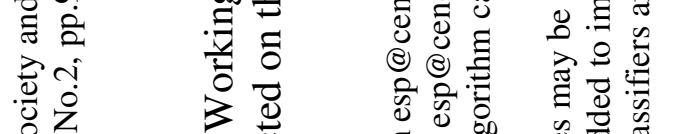

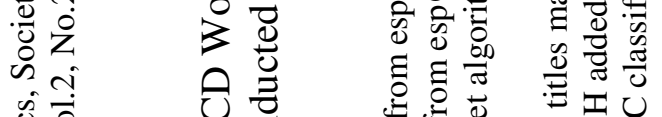

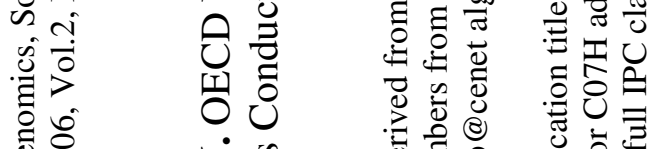

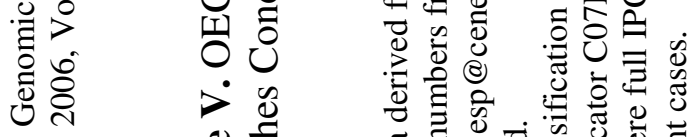

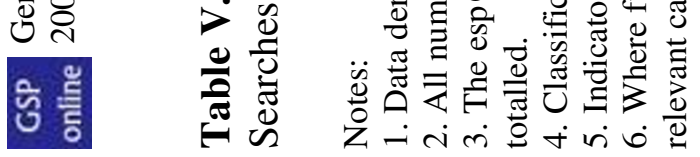

惫会

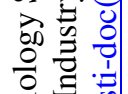

焉

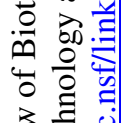

$\sum_{0}^{3}$ 至

ठำ

击 $\frac{0}{0}$ :

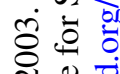

원

章要

可穿:

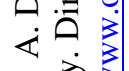

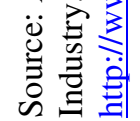

\begin{tabular}{|c|c|c|c|c|c|}
\hline 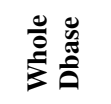 & $\begin{array}{l}\text { ơ } \\
\text { yे } \\
\text { in }\end{array}$ & $\begin{array}{l}\stackrel{0}{\circ} \\
\text { ले }\end{array}$ & $\begin{array}{l}\text { aे } \\
\text { aे }\end{array}$ & $\begin{array}{l}\text { iे } \\
\text { gे } \\
g\end{array}$ & $\begin{array}{l}\tilde{f} \\
\text { in } \\
\tilde{m}\end{array}$ \\
\hline 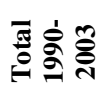 & 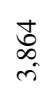 & $\begin{array}{l}\infty \\
\infty \\
\infty \\
\infty\end{array}$ & 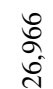 & $\begin{array}{l}\text { 今̂ } \\
\text { }\end{array}$ & 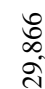 \\
\hline 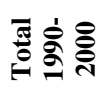 & $\stackrel{\infty}{\vec{i}}$ & $\underset{m}{\Xi}$ & $\frac{8}{6}$ & $\begin{array}{l}\stackrel{1}{2} \\
\text { aे }\end{array}$ & $\begin{array}{l} \pm \\
8 \\
\text { in }\end{array}$ \\
\hline 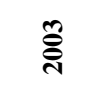 & ठీ & $\underset{\text { సె }}{ }$ & $\underset{\dot{s}}{\stackrel{\hat{n}}{m}}$ & $\underset{\text { ô }}{\text { i }}$ & 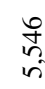 \\
\hline క్స్ & $\stackrel{\infty}{\stackrel{\infty}{+}}$ & స్ & $\begin{array}{l}n \\
n \\
n \\
q\end{array}$ & $\stackrel{\swarrow}{\cong}$ & $\frac{\infty}{n}$ \\
\hline క్స్ & $\stackrel{\sim}{\infty}$ & ల్లి & $\underset{\substack{n \\
q}}{m}$ & $\stackrel{2}{n}$ & $\stackrel{m}{;}$ \\
\hline సิ & 导 & ฉุ & $\underset{i}{\vec{i}}$ & $\underset{g}{g}$ & 今. \\
\hline ล & $n$ & $m$ & $\widetilde{\sigma}$ & $\mathcal{F}$ & $\curvearrowleft$ \\
\hline
\end{tabular}

\begin{tabular}{|c|c|c|c|c|c|}
\hline$\hat{\hat{\theta}}$ & $\stackrel{\text { ते }}{n}$ & f & $\begin{array}{l}\text { 定 } \\
\text { i. }\end{array}$ & 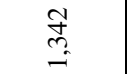 & 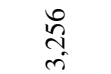 \\
\hline$\stackrel{\infty}{\circ}$ & $\stackrel{\sim}{\sim}$ & ळे & $\begin{array}{l}\underset{0}{0} \\
i \\
i\end{array}$ & 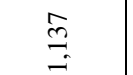 & $\begin{array}{l}n \\
\hat{\sigma} \\
i\end{array}$ \\
\hline$\hat{\hat{\sigma}}$ & $\stackrel{\infty}{\sim}$ & ల్రి & 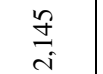 & $\bar{\alpha}$ & ڤे \\
\hline$\stackrel{2}{\circ}$ & q & $\underset{\Delta}{\stackrel{\Delta}{\Delta}}$ & $\underset{i}{i}$ & $\underset{\infty}{\infty}$ & 它 \\
\hline$\stackrel{\circ}{\circ}$ & $\stackrel{\infty}{\sim}$ & $\stackrel{\ominus}{\sim}$ & 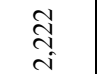 & $\stackrel{\circ}{ }$ & $\underset{\infty}{ు}$ \\
\hline 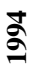 & 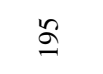 & वे & fo & $\bar{\infty}$ & : \\
\hline 气 & $\underline{\square}$ & $\ddot{\tilde{\gamma}}$ & $\stackrel{\varrho}{\beth}$ & $\Re$ & ते \\
\hline ڤี & $\stackrel{ \pm}{\Xi}$ & ले & $\stackrel{\leftrightarrow}{g}$ & $\begin{array}{l}\infty \\
i \\
i\end{array}$ & ڤิ \\
\hline बू & 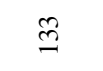 & I & $\stackrel{\circ}{\circ}$ & જे & $\stackrel{d}{\underline{O}}$ \\
\hline ప్ & $\stackrel{\beth}{\simeq}$ & 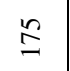 & $\stackrel{\infty}{n}$ & $\bar{\exists}$ & f \\
\hline U & $\underset{\frac{1}{4}}{\stackrel{8}{4}}$ & 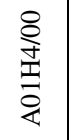 & 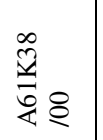 & 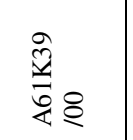 & 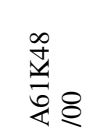 \\
\hline 气̆ & 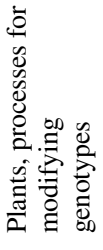 & 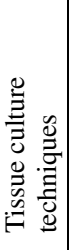 & 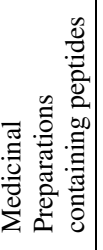 & 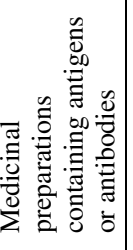 & 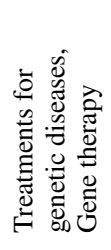 \\
\hline
\end{tabular}




\begin{tabular}{|c|c|c|c|c|c|c|c|c|c|c|c|c|}
\hline 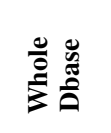 & & $\stackrel{0}{0}$ & $\frac{q}{m}$ & 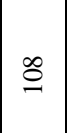 & $\vec{m}$ & ڤొ & $\begin{array}{l}\stackrel{0}{2} \\
\stackrel{2}{n} \\
\end{array}$ & 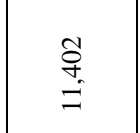 & $\begin{array}{l}\frac{n}{a} \\
\dot{f}\end{array}$ & $\stackrel{\infty}{\stackrel{\infty}{\stackrel{m}{r}}}$ & 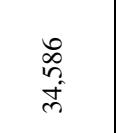 & $\begin{array}{l}8 \\
8 \\
\frac{1}{1} \\
\frac{1}{1}\end{array}$ \\
\hline 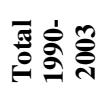 & & $\begin{array}{l}\text { qa } \\
\text { a } \\
\text { f }\end{array}$ & $\dddot{\dddot{q}}$ & $\tilde{m}$ & ø & $\stackrel{\Omega}{\sim}$ & 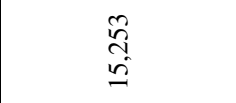 & $\begin{array}{l}\stackrel{0}{0} \\
\stackrel{n}{0} \\
\stackrel{0}{0}\end{array}$ & $\underset{f}{\stackrel{g}{+}}$ & $\stackrel{0}{\stackrel{0}{6}}$ & $\begin{array}{l}\stackrel{M}{A} \\
\stackrel{\sim}{d}\end{array}$ & $\begin{array}{l}\frac{8}{0} \\
\stackrel{\text { aे }}{0}\end{array}$ \\
\hline 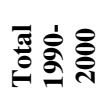 & & $\begin{array}{l}n \\
6 \\
n \\
n\end{array}$ & $\stackrel{f}{f}$ & $\stackrel{4}{A}$ & $\mathcal{F}$ & $\overline{\mathcal{F}}$ & $\underset{\substack{n \\
i}}{\stackrel{n}{n}}$ & $\underset{\mathfrak{g}}{\mathfrak{g}}$ & 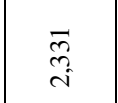 & $\frac{m}{n}$ & $\begin{array}{l}0 \\
0 \\
i \\
\end{array}$ & $\begin{array}{l}\stackrel{m}{\hat{z}} \\
\infty \\
\infty \\
\stackrel{0}{n}\end{array}$ \\
\hline ڤ్ڤે & & $\stackrel{\tilde{r}}{f}$ & $\tilde{\lambda}$ & $\nabla$ & 0 & q & 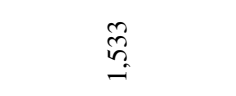 & $\stackrel{\circ}{:}$ & G & $\stackrel{0}{\mathscr{o}}$ & $\underset{\mathrm{d}}{\stackrel{\sim}{0}}$ & $\underset{\substack{\infty \\
\hat{\infty}}}{\infty}$ \\
\hline હิે & & $\vec{r}$ & 2 & $\mathrm{~N}$ & - & $\widetilde{\Xi}$ & 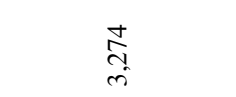 & $\begin{array}{l}+ \\
\substack{0 \\
i}\end{array}$ & $\hat{\infty}$ & $\stackrel{n}{\leftrightharpoons}$ & $\underset{\dot{m}}{\stackrel{\vec{\sigma}}{*}}$ & 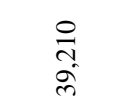 \\
\hline స్ํํ & & $\stackrel{్}{0}$ & $=$ & a & $\mathrm{N}$ & $\stackrel{\infty}{\curvearrowright}$ & $\underset{\substack{t \\
\infty \\
i}}{i}$ & $\underset{\substack{\text { di } \\
\text { in }}}{ }$ & mे & fơ & $\overrightarrow{\widetilde{\pi}}$ & $\begin{array}{c}\text { sे } \\
\text { in }\end{array}$ \\
\hline క్సે & & 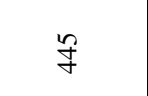 & $\simeq$ & 0 & N & $i n$ & $\underset{-}{\mathbb{S}}$ & 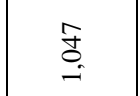 & శ్లి & $\stackrel{\infty}{\mathfrak{\sim}}$ & 吕 & 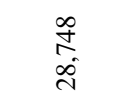 \\
\hline$\hat{\sigma}$ & & 㝏 & $\stackrel{\infty}{\simeq}$ & - & 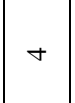 & ה & $\stackrel{n}{m}$ & $\stackrel{\mathscr{\sigma}}{\sigma}$ & $\underset{\sim}{\mathbb{N}}$ & $\stackrel{\infty}{\stackrel{\infty}{n}}$ & $\stackrel{\tilde{q}}{g}$ & $\begin{array}{l}\infty \\
\infty \\
\infty \\
\text { and }\end{array}$ \\
\hline$\stackrel{\infty}{\sigma}$ & & $\stackrel{\partial}{\vec{n}}$ & $=$ & - & - & $\vec{n}$ & ্ָড & 㕝 & $\hat{n}$ & $\tilde{i}$ & $\stackrel{\infty}{\infty}$ & 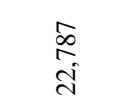 \\
\hline$\hat{2}$ & & $\stackrel{\infty}{\infty}$ & $\bar{\sim}$ & + & $\mathrm{N}$ & $R$ & $\begin{array}{l}\infty \\
\infty \\
\infty\end{array}$ & $\overline{\widehat{\sigma}}$ & $\tilde{n}$ & $\stackrel{\infty}{\stackrel{\infty}{n}}$ & సิ & $\begin{array}{l}\hat{y} \\
\stackrel{\infty}{0}\end{array}$ \\
\hline$\stackrel{2}{2}$ & & $\vec{m}$ & $\sqrt{2}$ & - & - & 8 & $\stackrel{\infty}{\infty}$ & $\mathscr{g}$ & : & ồ & $\stackrel{\text { fo }}{\stackrel{\text { f }}{-}}$ & 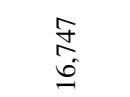 \\
\hline$\stackrel{\circ}{2}$ & & $\stackrel{m}{m}$ & ले & - & 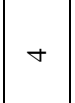 & 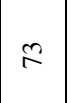 & $\stackrel{0}{0}$ & 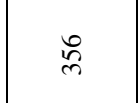 & $\underset{d}{\mathbb{d}}$ & 总 & $\stackrel{\circ}{\stackrel{m}{-}}$ & $\begin{array}{l}0 \\
\text { o } \\
\text { in }\end{array}$ \\
\hline$\stackrel{+}{\sigma}$ & & $\stackrel{\Re}{n}$ & in & in & 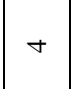 & 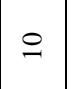 & ฉ & in & $\tilde{\tilde{n}}$ & $\nabla$ & $\underset{\sim}{\vec{g}}$ & 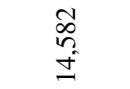 \\
\hline$\stackrel{\sigma}{\sigma}$ & & 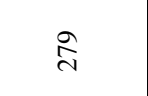 & 8 & $\mathrm{a}$ & in & $=$ & $\underset{J}{ \pm}$ & q & $\stackrel{\infty}{\sim}$ & $\tilde{\infty}$ & $\begin{array}{l}\stackrel{n}{2} \\
\stackrel{\sim}{-}\end{array}$ & 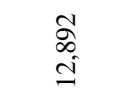 \\
\hline$\tilde{\sigma}$ & & : & $\vec{\infty}$ & $m$ & $r$ & $a$ & $\cong$ & iq & $\widehat{\Xi}$ & 8 & $\stackrel{\infty}{\stackrel{\infty}{\sim}}$ & $\stackrel{\infty}{\varrho}$ \\
\hline $\bar{\sigma}$ & & สี & f & $\mathrm{a}$ & $\infty$ & $a$ & $\bar{\sigma}$ & i & $\approx$ & $\stackrel{8}{8}$ & $\stackrel{+}{\stackrel{D}{=}}$ & $\begin{array}{l}\stackrel{8}{0} \\
\stackrel{0}{0}\end{array}$ \\
\hline 。 & & $\subseteq$ & $\ddot{\sigma}$ & + & 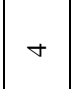 & - & $i$ & $\tilde{\lambda}$ & \& & q & $\begin{array}{l}\text { ָָ } \\
-\end{array}$ & $\begin{array}{l}\tilde{O} \\
\stackrel{0}{0}\end{array}$ \\
\hline U & Uू. & 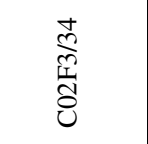 & \begin{tabular}{|l|}
$\overline{0}$ \\
0 \\
$\overline{8}$
\end{tabular} & $\begin{array}{l}\frac{m}{2} \\
0 \\
8 \\
8\end{array}$ & \begin{tabular}{|l|}
$\frac{n}{3}$ \\
0 \\
$\stackrel{8}{8}$ \\
8
\end{tabular} & 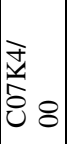 & $\begin{array}{l}\frac{\partial}{\vec{x}} \\
\frac{0}{8} 8\end{array}$ & $\begin{array}{l}\frac{0}{d} \\
\frac{0}{8} 8\end{array}$ & $\begin{array}{l}\overline{\bar{y}} \\
\frac{3}{8} 8\end{array}$ & $\begin{array}{l}\frac{\bar{v}}{\Delta} \\
\frac{\vec{y}}{8} 8\end{array}$ & 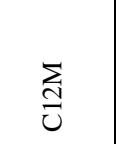 & ż \\
\hline 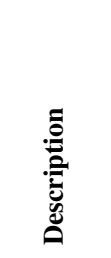 & & 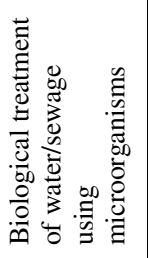 & 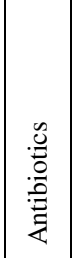 & 表 & 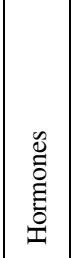 & : & 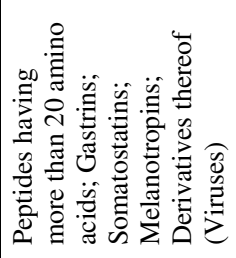 & 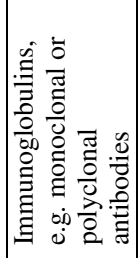 & 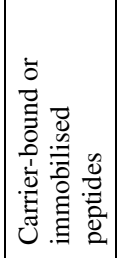 & 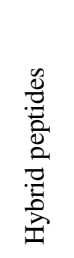 & 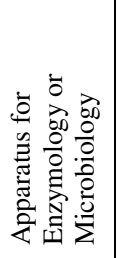 & 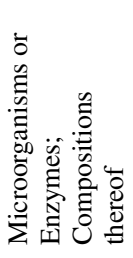 \\
\hline
\end{tabular}




\begin{tabular}{|c|c|c|c|c|c|c|c|}
\hline 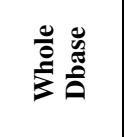 & $\begin{array}{l}\frac{8}{8} \\
\frac{8}{7}+\frac{1}{7}\end{array}$ & $\begin{array}{l}\frac{8}{8} \\
\frac{8}{7}+\frac{1}{7}\end{array}$ & ले & ళ్ల & $\begin{array}{l}\frac{a}{\bar{\partial}} \\
\text { के }\end{array}$ & d8 & \pm \\
\hline 至高咅言 & 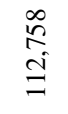 & $\begin{array}{l}\text { 营 } \\
\text { d् }\end{array}$ & $\frac{b}{\infty}$ & 总 & 帘 & $\pi$ & $\underline{n}$ \\
\hline 焉高高 & 爰 & 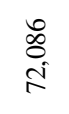 & 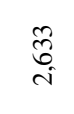 & ప్రి & 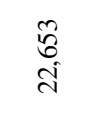 & $=$ & 。 \\
\hline 䓂 & 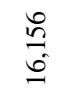 & $\begin{array}{l}9 \\
0 \\
2\end{array}$ & : & 8 & 愈 & - & a \\
\hline స్్ㅀ & 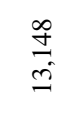 & 文 & $\Xi$ & $D$ & $\begin{array}{c}\infty \\
\vdots \\
0 \\
0\end{array}$ & $a$ & $m$ \\
\hline 言 & 趈 & 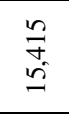 & $\stackrel{m}{m}$ & בे & $\frac{8}{8}$ & a & + \\
\hline 㐔 & 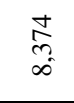 & 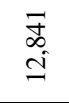 & $\stackrel{8}{9}$ & $\overrightarrow{\vec{d}}$ & $\begin{array}{l}\infty \\
\text { dै } \\
\text { हैं }\end{array}$ & - & - \\
\hline gे & $\frac{v_{\infty}^{\prime}}{\infty}$ & 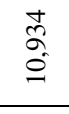 & శ్తి & $\dddot{y}$ & $\frac{ \pm}{\vec{c}}$ & - & - \\
\hline$\stackrel{\infty}{\circ}$ & 㐫 & : & $\stackrel{\circ}{~}$ & $\stackrel{\check{0}}{\circ}$ & 虽 & 0 & $\circ$ \\
\hline 重 & 䍂 & 壾 & E & $\underline{\Phi}$ & $\underset{j}{ \pm}$ & $a$ & 0 \\
\hline$\stackrel{\circ}{\circ}$ & \&్తి & 莺 & ลิ & $\nexists$ & ईેे & 0 & 0 \\
\hline$\frac{0}{g}$ & $\stackrel{R}{N}$ & 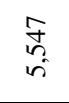 & 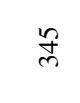 & $\stackrel{\Xi}{\Xi}$ & 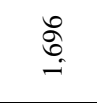 & 0 & - \\
\hline 壴 & 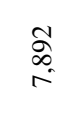 & 另 & 莒 & ฐి & $\stackrel{\text { Dे }}{=}$ & 0 & - \\
\hline 要 & 范 & 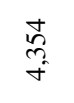 & ন & $\Xi$ & $\stackrel{\infty}{q}$ & $m$ & - \\
\hline 亏ี & : & 总 & : & $\infty$ & E & 0 & $\circ$ \\
\hline$\overline{\mathrm{g}}$ & 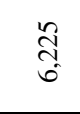 & 釆 & 莒 & $\infty$ & 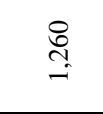 & $\sim$ & 0 \\
\hline 吾 & $\frac{9}{\bar{\sigma}}$ & 釆 & $F$ & $\cong$ & 志 & $\infty$ & - \\
\hline 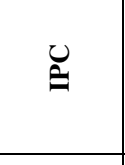 & ปิ & gี & $\stackrel{\mathscr{U}}{\tilde{u}}$ & 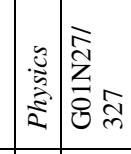 & $\begin{array}{l}\overline{\bar{x}} \\
\bar{z}_{\bar{z}}\end{array}$ & 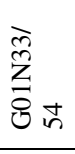 & 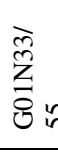 \\
\hline 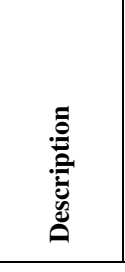 & & & & & & & \\
\hline
\end{tabular}




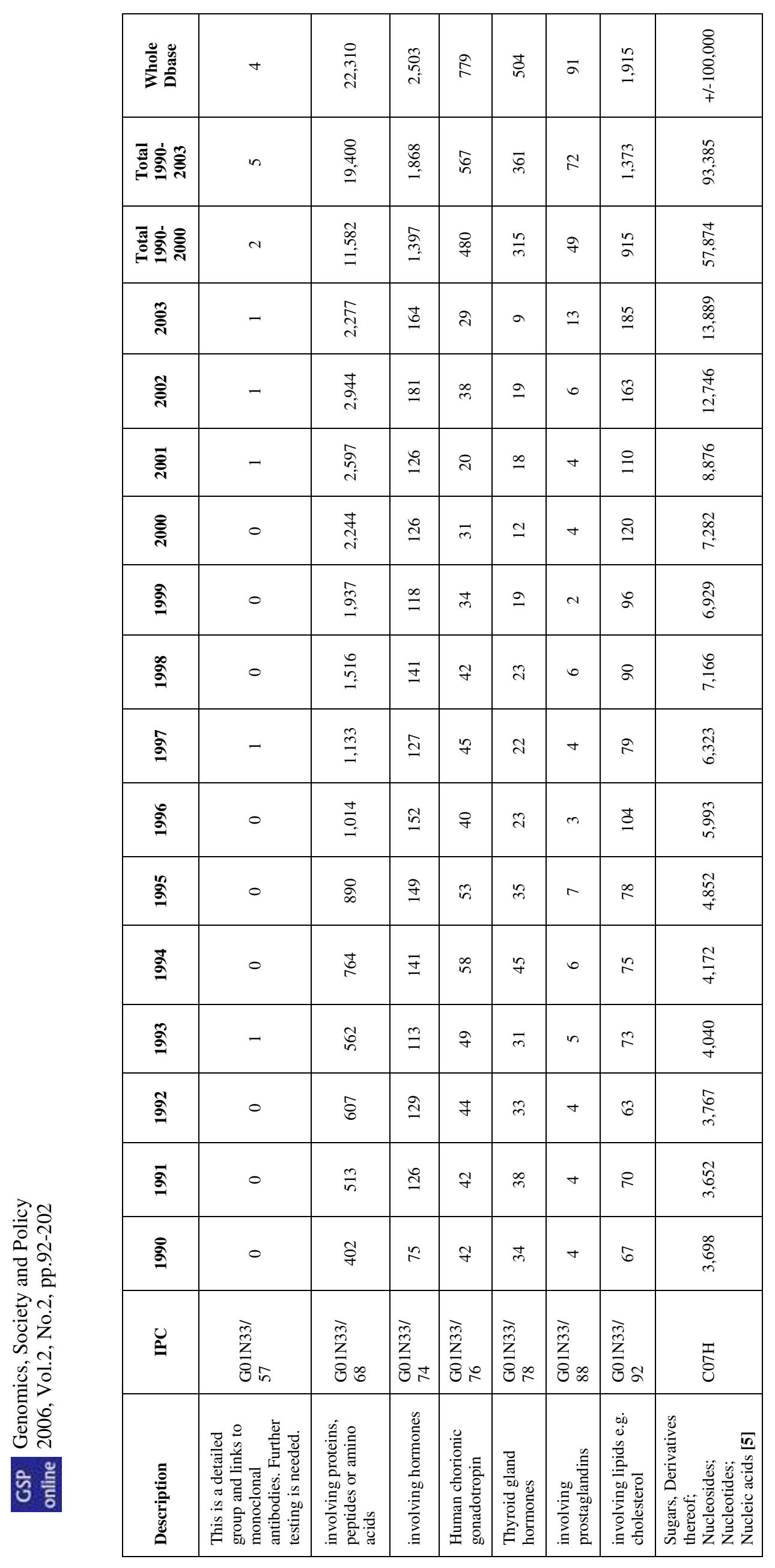


Table VI. Indicators for Diseases (A61P) Searches Conducted August 2005

Notes:

1. Data derived from esp@cenet http://ep.espacenet.com/.

2. All numbers from esp@ @enet are approximate and refer to patent publication counts.

3. The esp@ cenet algorithm cannot produce results beyond 100,000. Individual results on or close to 100,000 (e.g. A61) reflect this limitation. These numbers should not be totalled.

4. Classification titles may be abbreviated. For further detail consult the International Patent

Classification (IPC). http://www.wipo.int/classifications/ipc/en/.

5. Indicator under A61P were introduced in the year 2000 and their international use by patent offices worldwide may vary.

6. Patent examiners will generally award a classifier as A61P1 rather than the full A61P1/00. Exclude the /00 for more accurate returns in relevant cases.

\begin{tabular}{|c|c|c|}
\hline Diseases/Disorders & IPC & $\begin{array}{l}\text { espacenet coverage } \\
\text { whole database }\end{array}$ \\
\hline Drugs for disorders of the alimentary tract or the digestive system & $\mathrm{A} 61 \mathrm{P} 1 / 00$ & 20,178 \\
\hline Stomatological preparations, e.g. drugs for caries, aphtae, periodontitis & A61P1/02 & 2,382 \\
\hline $\begin{array}{l}\text { for ulcers, gastritis or reflux esophagitis, e.g. antacids, inhibitors ofacid secretion, } \\
\text { mucosal protectants }\end{array}$ & A61P1/04 & 6,309 \\
\hline Anti-spasmodics, e.g. drugs for colics, esophagic dyskinesia & A61P1/06 & 283 \\
\hline for nausea, cinetosis or vertigo; Antiemetics & A61P1/08 & 977 \\
\hline .Laxatives & A61P1/10 & 646 \\
\hline .Antidiarrhoeals & A61P1/12 & 965 \\
\hline $\begin{array}{l}\text { Prodigestives, e.g. acids, enzymes, appetite stimulants, antidyspeptics, tonics, } \\
\text { antiflatulents }\end{array}$ & A61P1/14 & 2,069 \\
\hline $\begin{array}{l}\text { for liver or gallbladder disorders, e.g. hepatoprotective agents, cholagogues, } \\
\text { litholytics }\end{array}$ & $\mathrm{A} 61 \mathrm{P} 1 / 16$ & 4,978 \\
\hline for pancreatic disorders, e.g. pancreatic enzymes & $\mathrm{A} 61 \mathrm{P} 1 / 18$ & 1,079 \\
\hline Drugs for disorders of the metabolism (of the blood or the extracellular fluid 7/00) & $\mathrm{A} 61 \mathrm{P} 3 / 00$ & 23,249 \\
\hline Nutrients, e.g. vitamins, minerals & $\mathrm{A} 61 \mathrm{P} 3 / 02$ & 1,738 \\
\hline .Anorexiants; Antiobesity agents & A61P3/04 & 5,887 \\
\hline .Antihyperlipidemics & $\mathrm{A} 61 \mathrm{P} 3 / 06$ & 5,890 \\
\hline for glucose homeostasis (pancreatic hormones 5/48) & $\mathrm{A} 61 \mathrm{P} 3 / 08$ & 563 \\
\hline .. for hyperglycaemia, e.g. antidiabetics & $\mathrm{A} 61 \mathrm{P} 3 / 10$ & 12,358 \\
\hline for electrolyte homeostasis & $\mathrm{A} 61 \mathrm{P} 3 / 12$ & 232 \\
\hline .. for calcium homeostasis & $\mathrm{A} 61 \mathrm{P} 3 / 14$ & 612 \\
\hline Drugs for disorders of the endocrine system & A61P5/00 & 6,988 \\
\hline .of the hypothalamic hormones, e.g. TRH, GnRH, CRH, GRH, somatostatin & A61P5/02 & 285 \\
\hline $\begin{array}{l}. . \text { for decreasing, blocking or antagonising the activity of the hypothalamic } \\
\text { hormones }\end{array}$ & A61P5/04 & 106 \\
\hline .of the anterior pituitary hormones, e.g. TSH, ACTH, FSH, LH, PRL, GH & $\mathrm{A} 61 \mathrm{P} 5 / 06$ & 351 \\
\hline $\begin{array}{l}\text {.. for decreasing, blocking or antagonising the activity of the anteriorpituitary } \\
\text { hormones }\end{array}$ & A61P5/08 & 67 \\
\hline .of the posterior pituitary hormones, e.g. oxytocin, ADH & A61P5/10 & 158 \\
\hline $\begin{array}{l}\text {.. for decreasing, blocking or antagonising the activity of the posteriorpituitary } \\
\text { hormones }\end{array}$ & $\mathrm{A} 61 \mathrm{P} 5 / 12$ & 31 \\
\hline .of the thyroid hormones, e.g. T3, T4 & A61P5/14 & 613 \\
\hline .. for decreasing, blocking or antagonising the activity of the thyroid hormones & A61P5/16 & 147 \\
\hline .of the parathyroid hormones & A61P5/18 & 172 \\
\hline .. for decreasing, blocking or antagonising the activity of PTH & $\mathrm{A} 61 \mathrm{P} 5 / 20$ & 35 \\
\hline .. for decreasing, blocking or antagonising the activity of calcitonin & $\mathrm{A} 61 \mathrm{P} 5 / 22$ & 18 \\
\hline .of the sex hormones & $\mathrm{A} 61 \mathrm{P} 5 / 24$ & 615 \\
\hline .. Androgens & A61P5/26 & 239 \\
\hline .. Antiandrogens & $\mathrm{A} 61 \mathrm{P} 5 / 28$ & 206 \\
\hline .. Oestrogens & $\mathrm{A} 61 \mathrm{P} 5 / 30$ & 552 \\
\hline
\end{tabular}




\begin{tabular}{|c|c|c|}
\hline Diseases/Disorders & IPC & $\begin{array}{l}\text { espacenet coverage } \\
\text { whole database }\end{array}$ \\
\hline .. Antioestrogens & A61P5/32 & 238 \\
\hline .. Gestagens & A61P5/34 & 57 \\
\hline .. Antigestagens & A61P5/36 & 50 \\
\hline .of the suprarenal hormones & A61P5/38 & 162 \\
\hline .. Mineralocorticosteroids, e.g. aldosterone; Drugs & $\mathrm{A} 61 \mathrm{P} 5 / 40$ & 60 \\
\hline .. for decreasing, blocking or antagonising the activity of mineralocorticosteroids & A61P5/42 & 40 \\
\hline $\begin{array}{l}\text {.. Glucocorticosteroids; Drugs increasing or potentiating the activity of } \\
\text { glucocorticosteroids }\end{array}$ & A61P5/44 & 91 \\
\hline .. for decreasing, blocking or antagonising the activity of glucocorticosteroids & A61P5/46 & 48 \\
\hline of the pancreatic hormones & A61P5/48 & 571 \\
\hline .. for increasing or potentiating the activity of insulin & A61P5/50 & 561 \\
\hline Drugs for disorders of the blood or the extracellular fluid & $\mathrm{A} 61 \mathrm{P} 7 / 00$ & 11,600 \\
\hline .Antithrombotic agents; Anticoagulants; Platelet aggregation inhibitors & $\mathrm{A} 61 \mathrm{P} 7 / 02$ & 5,411 \\
\hline .Antihaemorrhagics; Procoagulants; Haemostatatic agents; Antifibrinolyticagents & $\mathrm{A} 61 \mathrm{P} 7 / 04$ & 1,561 \\
\hline .Antianaemics & A61P7/06 & 1,493 \\
\hline $\begin{array}{l}\text { Plasma substitutes; Perfusion solutions; Dialytics or haemodialytics; Drugs for } \\
\text { electrolytic or acid-base disorders, e.g. hypovolemic shock (artificial tears } 81\end{array}$ & $\mathrm{~A} 61 \mathrm{P} 7 / 08$ & 391 \\
\hline .Antioedematous agents; Diuretics & $\mathrm{A} 61 \mathrm{P} 7 / 10$ & 547 \\
\hline .Antidiuretics, e.g. drugs for diabetes insipidus (ADH5/10) & $\mathrm{A} 61 \mathrm{P} 7 / 12$ & 165 \\
\hline Drugs for disorders of the cardiovascular system & A61P9/00 & 12,515 \\
\hline .Non-specific cardiovascular stimulants, e.g. drugs for syncope, antihypotensives & A61P9/02 & 792 \\
\hline .Inotropic agents, i.e. stimulants of cardiac contraction; Drugs for heart failure & A61P9/04 & 2,162 \\
\hline .Antiarrhythmics & A61P9/06 & 1,600 \\
\hline .Vasodilators for multiple indications & A61P9/08 & 1,116 \\
\hline $\begin{array}{l}\text { for treating ischaemic or atherosclerotic diseases, e.g. antianginal drugs, coronary } \\
\text { vasodilators, drugs for myocardial infarction, retinopathy, arteriosclerosis }\end{array}$ & A61P9/10 & 30,145 \\
\hline .Antihypertensives & $\mathrm{A} 61 \mathrm{P} 9 / 12$ & 5,368 \\
\hline $\begin{array}{l}. \text { Vasoprotectives; Antihaemorrhoidals; Drugs for varicose therapy; } \\
\text { Capillarystabilisers }\end{array}$ & A61P9/14 & 1,048 \\
\hline Drugs for disorders of the respiratory system & A61P11/00 & 15,174 \\
\hline . Nasal agents, e.g. decongestants & A61P11/02 & 1,481 \\
\hline for throat disorders & A61P11/04 & 568 \\
\hline Antiasthmatics & A61P11/06 & 7,321 \\
\hline . Bronchodilators & A61P11/08 & 927 \\
\hline . Expectorants & A61P11/10 & 340 \\
\hline . Mucolytics & A61P11/12 & 96 \\
\hline Antitussive agents & A61P11/14 & 770 \\
\hline . Central respiratory analeptics & A61P11/16 & 147 \\
\hline Drugs for disorders of the urinary system (diuretics $7 / 10$ ) & $\mathrm{A} 61 \mathrm{P} 13 / 00$ & 9,300 \\
\hline . of urine or of the urinary tract, e.g. urine acidifiers & $\mathrm{A} 61 \mathrm{P} 13 / 02$ & 1,065 \\
\hline for urolithiasis & A61P13/04 & 188 \\
\hline . Anti-spasmodics & A61P13/06 & 32 \\
\hline of the prostate & A61P13/08 & 1,662 \\
\hline of the bladder & $\mathrm{A} 61 \mathrm{P} 13 / 10$ & 940 \\
\hline of the kidneys & $\mathrm{A} 61 \mathrm{P} 13 / 12$ & 4,859 \\
\hline $\begin{array}{l}\text { Drugs for genital or sexual disorders (for disorders of sex hormones } \\
5 / 24 \text { );Contraceptives }\end{array}$ & A61P15/00 & 9,745 \\
\hline for disorders of the vagina & A61P15/02 & 528 \\
\hline . for inducing labour or abortion; Uterotonics & $\mathrm{A} 61 \mathrm{P} 15 / 04$ & 96 \\
\hline Antiabortive agents; Labour repressants & A61P15/06 & 508 \\
\hline $\begin{array}{l}\text {. for gonadal disorders or for enhancing fertility, e.g. inducers of ovulat } \\
\text { spermatogenesis }\end{array}$ & A61P15/08 & 1,528 \\
\hline
\end{tabular}




\begin{tabular}{|c|c|c|}
\hline Diseases/Disorders & IPC & $\begin{array}{l}\text { espacenet coverage } \\
\text { whole database }\end{array}$ \\
\hline for impotence & A61P15/10 & 1,838 \\
\hline for climacteric disorders & A61P15/12 & 716 \\
\hline . for lactation disorders, e.g. galactorrhoea & A61P15/14 & 228 \\
\hline . Masculine contraceptives & $\mathrm{A} 61 \mathrm{P} 15 / 16$ & 251 \\
\hline . Feminine contraceptives & $\mathrm{A} 61 \mathrm{P} 15 / 18$ & 788 \\
\hline Drugs for dermatological disorders & A61P17/00 & 20,973 \\
\hline . for treating wounds, ulcers, burns, scars, keloids, or the like & $\mathrm{A} 61 \mathrm{P} 17 / 02$ & 4,927 \\
\hline . Antipruritics & A61P17/04 & 1,210 \\
\hline Antipsoriatics & A61P17/06 & 4,712 \\
\hline . Antiseborrheics & A61P17/08 & 305 \\
\hline . Anti-acne agents & A61P17/10 & 1,136 \\
\hline . Keratolytics, e.g. wart or anti-corn preparations & A61P17/12 & 331 \\
\hline for baldness or alopecia & A61P17/14 & 1,775 \\
\hline . Emollients or protectives, e.g. against radiation & $\mathrm{A} 61 \mathrm{P} 17 / 16$ & 2,471 \\
\hline Drugs for skeletal disorders & A61P19/00 & 15,920 \\
\hline . for joint disorders, e.g. arthritis, arthrosis & A61P19/02 & 8,063 \\
\hline for non-specific disorders of the connective tissue & A61P19/04 & 522 \\
\hline . Antigout agents, e.g. antihyperuricemic or uricosuric agents & A61P19/06 & 598 \\
\hline . for bone diseases, e.g. rachitism, Paget's disease & A61P19/08 & 2,959 \\
\hline . . for osteoporosis & A61P19/10 & 4,648 \\
\hline Drugs for disorders of the muscular or neuromuscular system & A61P21/00 & 3,499 \\
\hline . Muscle relaxants, e.g. for tetanus or cramps & A61P21/02 & 519 \\
\hline for myasthenia gravis & A61P21/04 & 994 \\
\hline . Anabolic agents (androgens 5/26) & A61P21/06 & 46 \\
\hline Anaesthetics & A61P23/00 & 975 \\
\hline . Local anaesthetics & A61P23/02 & 330 \\
\hline Drugs for disorders of the nervous system & $\mathrm{A} 61 \mathrm{P} 25 / 00$ & 37,643 \\
\hline . for peripheral neuropathies & $\mathrm{A} 61 \mathrm{P} 25 / 02$ & 1,831 \\
\hline . Centrally acting analgesics, e.g. opioids & $\mathrm{A} 61 \mathrm{P} 25 / 04$ & 3,841 \\
\hline . Antimigraine agents & $\mathrm{A} 61 \mathrm{P} 25 / 06$ & 2,238 \\
\hline Antiepileptics; Anticonvulsants & A61P25/08 & 2,532 \\
\hline . for petit-mal & A61P25/10 & 44 \\
\hline .. for grand-mal & A61P25/12 & 30 \\
\hline . for treating abnormal movements, e.g. chorea, dyskinesia & $\mathrm{A} 61 \mathrm{P} 25 / 14$ & 2,100 \\
\hline . Anti-Parkinson drugs & A61P25/16 & 4,548 \\
\hline . Antipsychotics, i.e. neuroleptics; Drugs for mania or schizophrenia & $\mathrm{A} 61 \mathrm{P} 25 / 18$ & 4,151 \\
\hline . Hypnotics; Sedatives & $\mathrm{A} 61 \mathrm{P} 25 / 20$ & 1,844 \\
\hline Anxiolytics & $\mathrm{A} 61 \mathrm{P} 25 / 22$ & 3,065 \\
\hline . Antidepressants & $\mathrm{A} 61 \mathrm{P} 25 / 24$ & 5,059 \\
\hline Psychostimulants, e.g. nicotine, cocaine & A61P25/26 & 153 \\
\hline $\begin{array}{l}\text { for treating neurodegenerative disorders of the central nervous system, e.g. } \\
\text { nootropic agents, }\end{array}$ & $\mathrm{A} 61 \mathrm{P} 25 / 28$ & 12,141 \\
\hline for treating abuse or dependence & $\mathrm{A} 61 \mathrm{P} 25 / 30$ & 1,278 \\
\hline .. Alcohol-abuse & A61P25/32 & 912 \\
\hline .. Tobacco-abuse & $\mathrm{A} 61 \mathrm{P} 25 / 34$ & 564 \\
\hline . . Opioid-abuse & A61P25/36 & 689 \\
\hline Drugs for disorders of the senses & A61P27/00 & 9,213 \\
\hline Ophthalmic agents & A61P27/02 & 5,465 \\
\hline . . Artificial tears; Irrigation solutions & A61P27/04 & 256 \\
\hline .. Antiglaucoma agents or miotics & A61P27/06 & 1,857 \\
\hline
\end{tabular}




\begin{tabular}{|c|c|c|}
\hline Diseases/Disorders & IPC & $\begin{array}{c}\text { espacenet coverage } \\
\text { whole database }\end{array}$ \\
\hline .. Mydriatics or cycloplegics & A61P27/08 & 18 \\
\hline .. for accommodation disorders, e.g. myopia & A61P27/10 & 127 \\
\hline . . for cataracts & A61P27/12 & 531 \\
\hline .. Decongestants or antiallergics & A61P27/14 & 549 \\
\hline Otologicals & A61P27/16 & 1,290 \\
\hline Non-central analgesic, antipyretic or anti-inflammatory agents & A61P29/00 & 22,042 \\
\hline Anti-infectives, i.e. antibiotics, antiseptics, chemotherapeutics & A61P31/00 & 31,939 \\
\hline . Local antiseptics & A61P31/02 & 465 \\
\hline . Antibacterial agents & A61P31/04 & 11,537 \\
\hline .. for tuberculosis & A61P31/06 & 705 \\
\hline . . for leprosy & A61P31/08 & 109 \\
\hline . Antimycotics & A61P31/10 & 2,960 \\
\hline . Antivirals & A61P31/12 & 7,788 \\
\hline .. for RNA viruses & A61P31/14 & 1,669 \\
\hline$\ldots$ for influenza or rhinoviruses & A61P31/16 & 852 \\
\hline ... for HIV & A61P31/18 & 5,269 \\
\hline . . for DNA viruses & A61P31/20 & 822 \\
\hline$\ldots$ for herpes viruses & A61P31/22 & 1,394 \\
\hline Antiparasitic agents & A61P33/00 & 3,905 \\
\hline Antiprotozoals, e.g. for leishmaniasis, trichomoniasis, toxoplasmosis & $\mathrm{A} 61 \mathrm{P} 33 / 02$ & 963 \\
\hline . Amoebicides & $\mathrm{A} 61 \mathrm{P} 33 / 04$ & 47 \\
\hline . . Antimalarials & $\mathrm{A} 61 \mathrm{P} 33 / 06$ & 815 \\
\hline . . for Pneumocystis carinii & $\mathrm{A} 61 \mathrm{P} 33 / 08$ & 44 \\
\hline . Anthelmintics & A61P33/10 & 380 \\
\hline . . Schistosomicides & $\mathrm{A} 61 \mathrm{P} 33 / 12$ & 83 \\
\hline . Ectoparasiticides, e.g. scabicides & A61P33/14 & 315 \\
\hline Antineoplastic agents & A61P35/00 & 40,859 \\
\hline specific for leukemia & $\mathrm{A} 61 \mathrm{P} 35 / 02$ & 2,289 \\
\hline specific for metastasis & A61P35/04 & 2,068 \\
\hline Drugs for immunological or allergic disorders & A61P37/00 & 21,448 \\
\hline Immunomodulators & A61P37/02 & 4,726 \\
\hline . . Immunostimulants & A61P37/04 & 3,014 \\
\hline . . Immunosuppressants, e.g. drugs for graft rejection & A61P37/06 & 4,991 \\
\hline . Antiallergic agents (antiasthmatic agents 11/06; ophthalmic antiallergics27/14) & A61P37/08 & 5,850 \\
\hline General protective or antinoxious agents & A61P39/00 & 2,871 \\
\hline Antidotes & A61P39/02 & 635 \\
\hline . Chelating agents & A61P39/04 & 75 \\
\hline Free radical scavengers or antioxidants & A61P39/06 & 1,432 \\
\hline $\begin{array}{l}\text { Drugs used in surgical methods, e.g. surgery adjuvants for preventing adhesion or } \\
\text { for vitreum substitution }\end{array}$ & A61P41/00 & 708 \\
\hline Drugs for specific purposes, not provided for in groups $1 / 00$ to $41 / 00$ & $\mathrm{~A} 61 \mathrm{P} 43 / 00$ & 27,938 \\
\hline
\end{tabular}




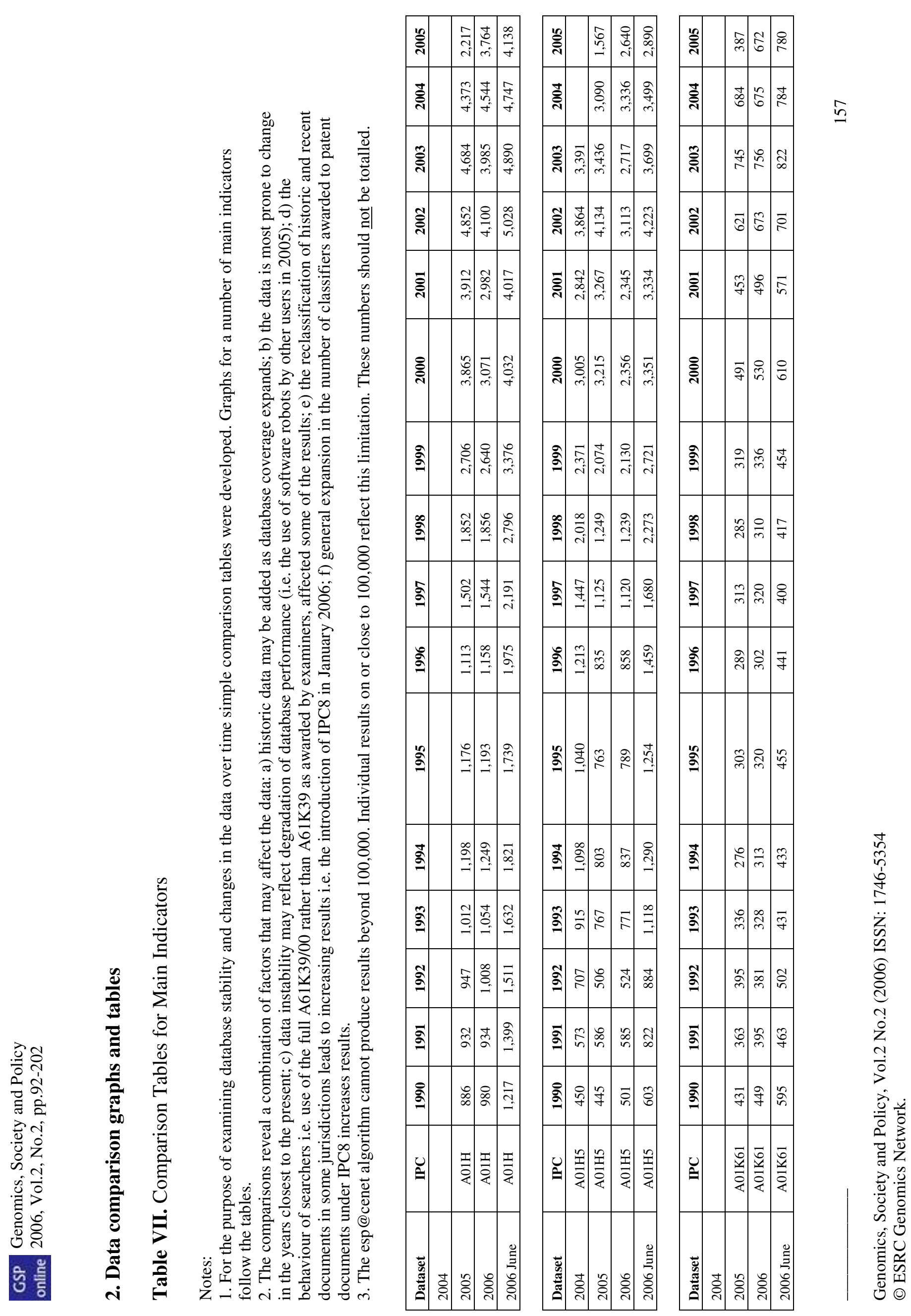




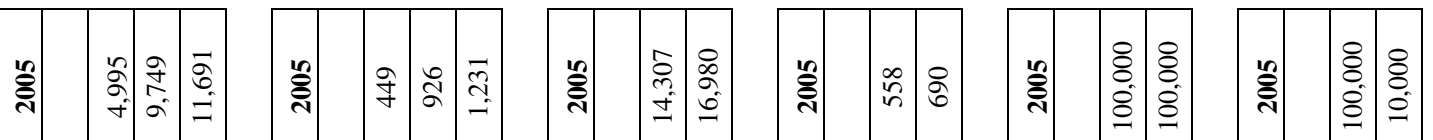

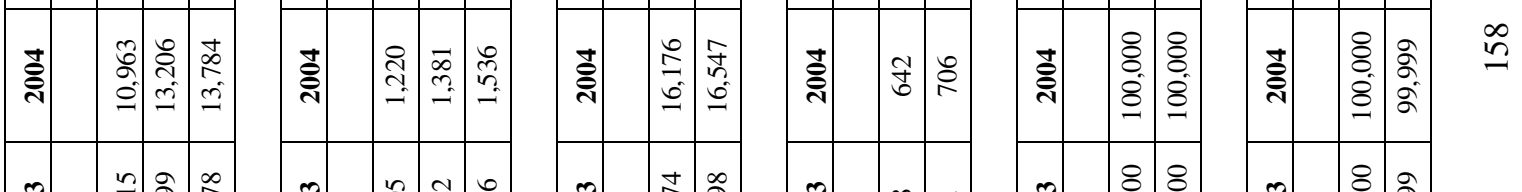

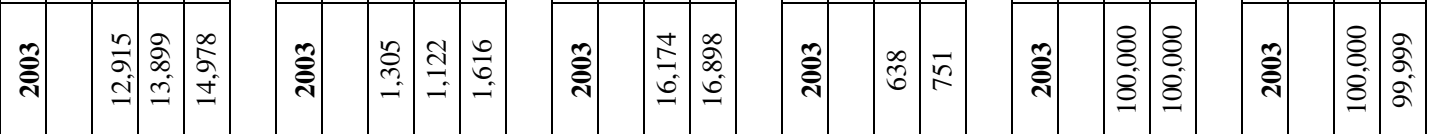

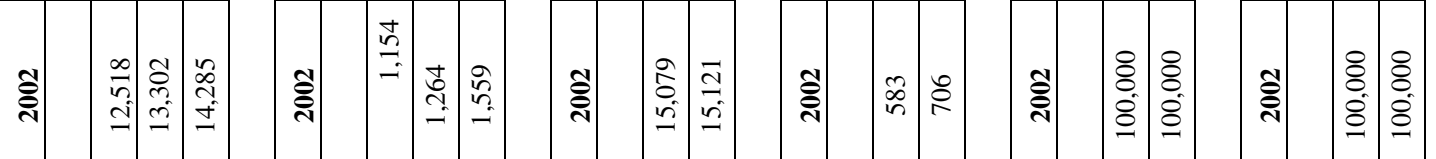

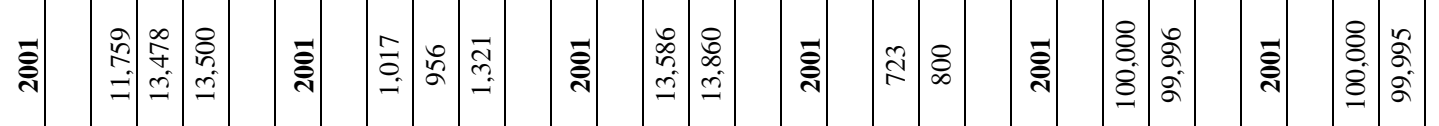

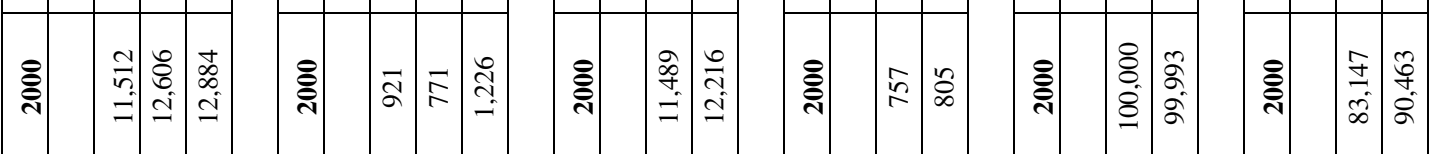

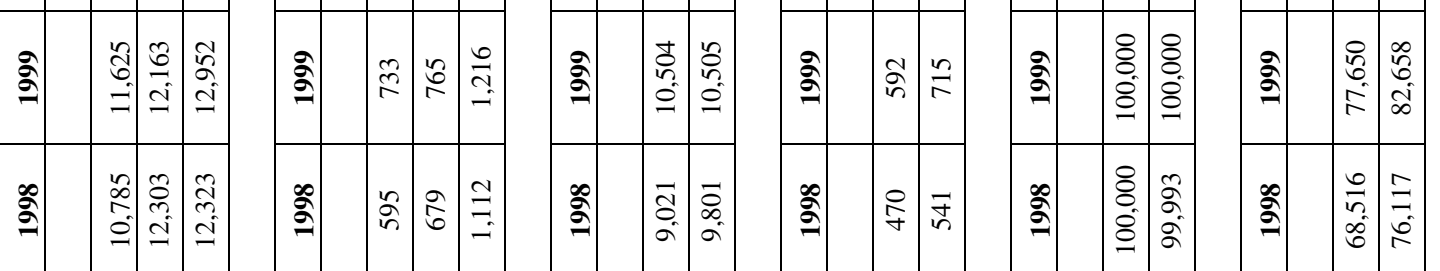

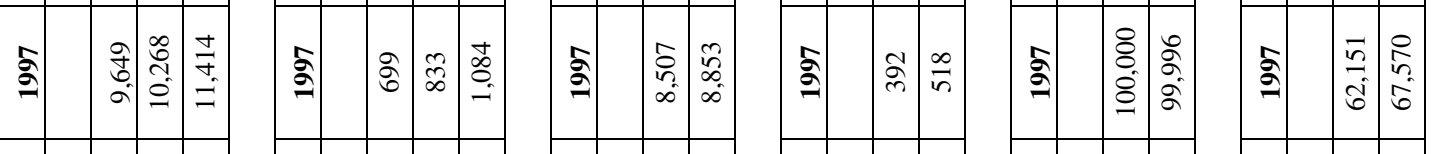

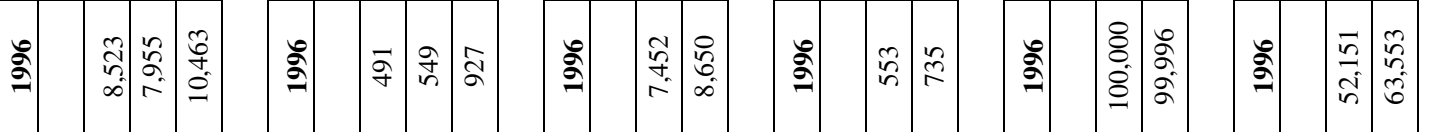

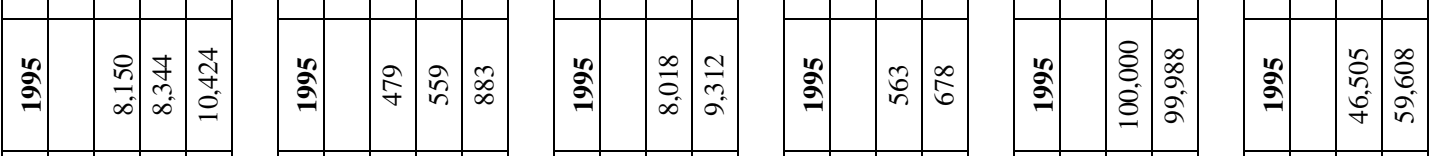

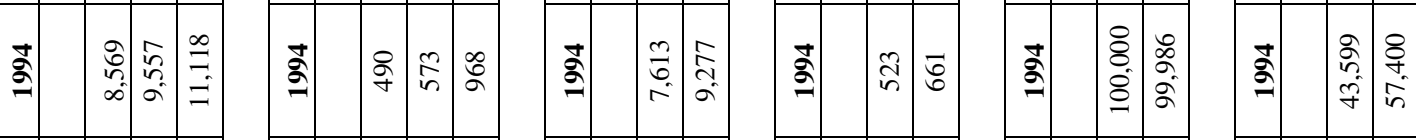

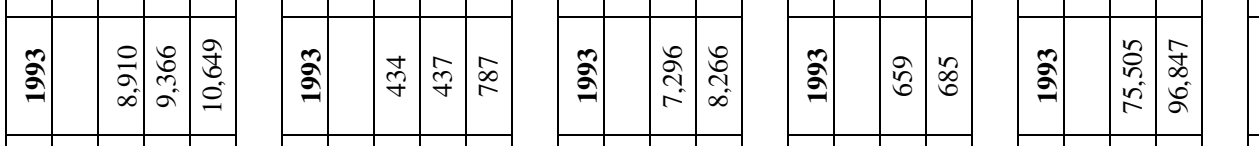

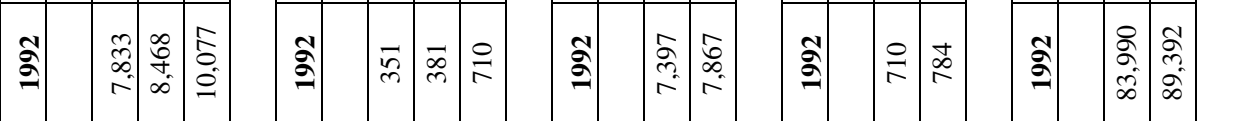

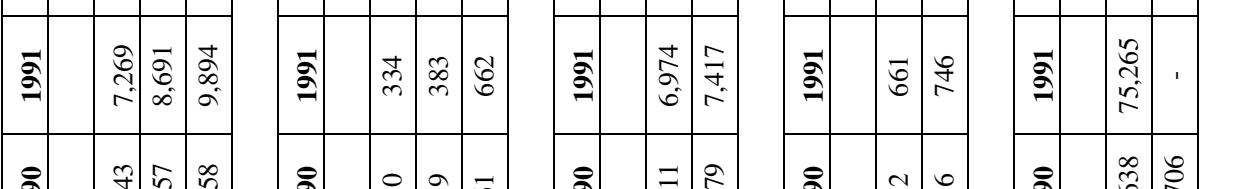

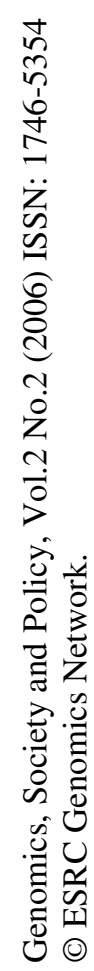




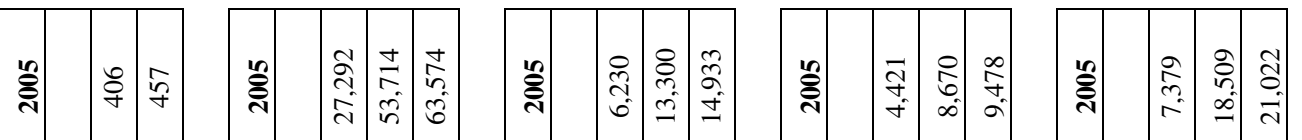

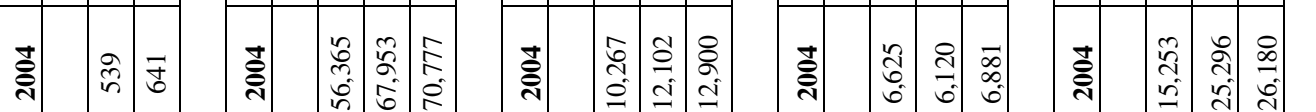

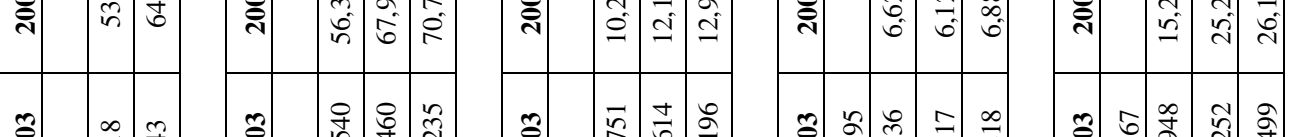

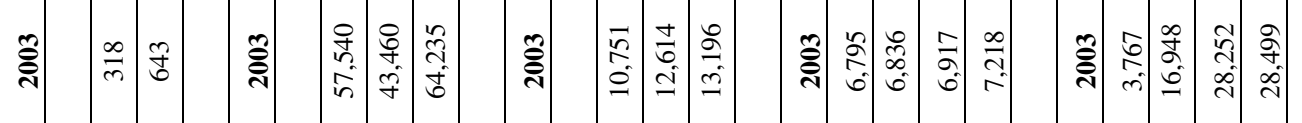

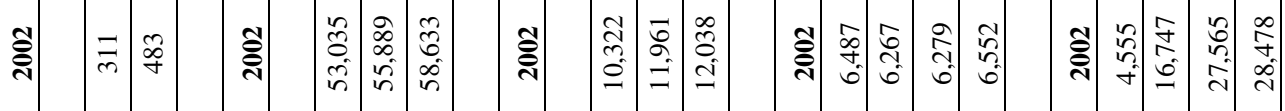

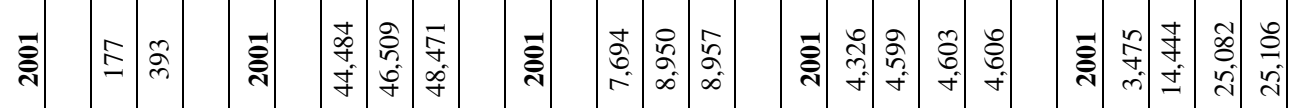

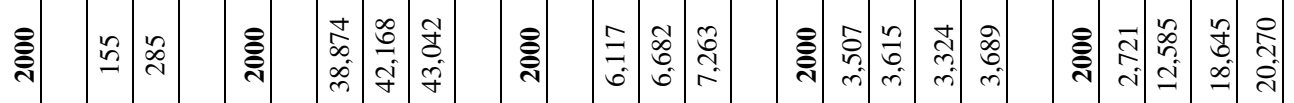

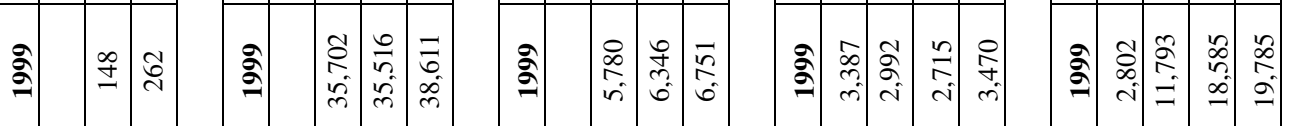

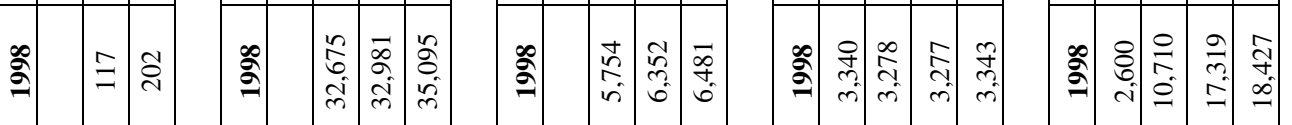

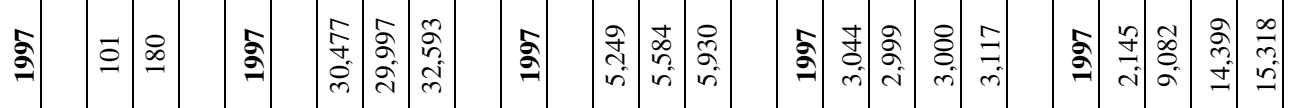

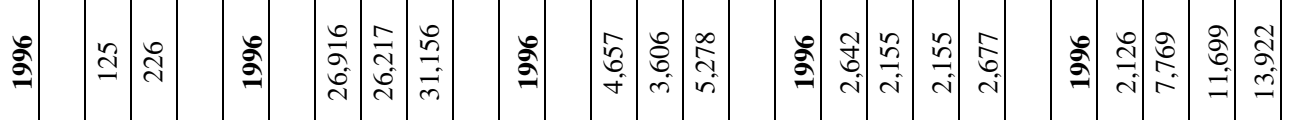

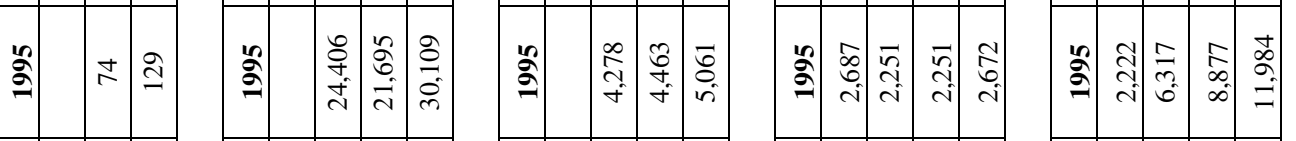

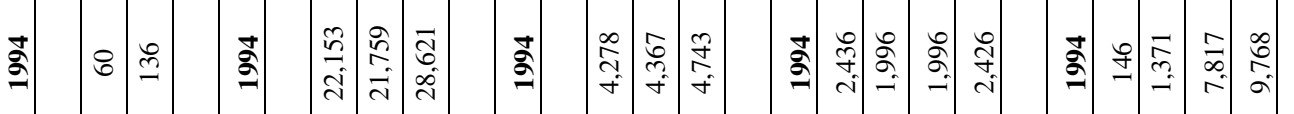

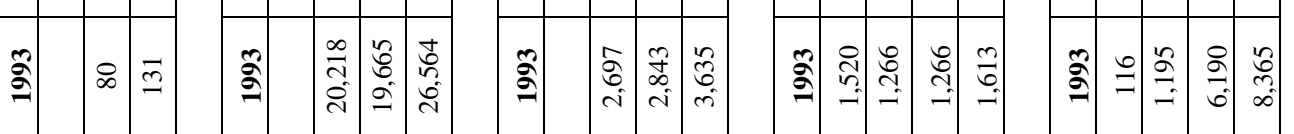

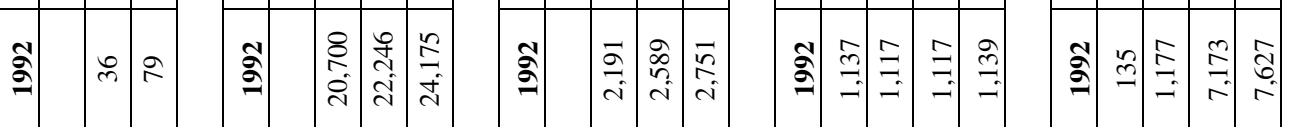

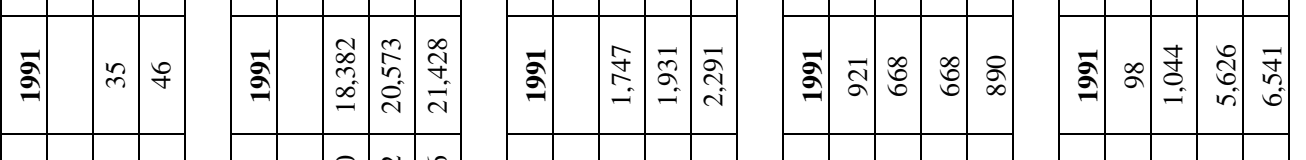

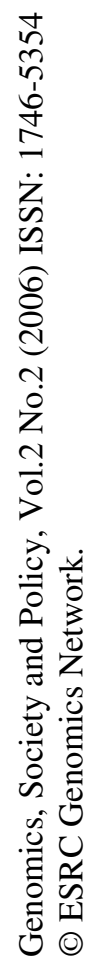




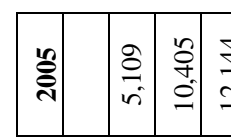

言

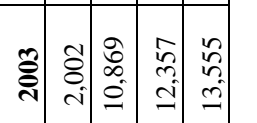

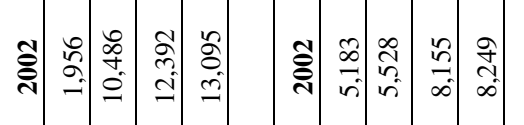

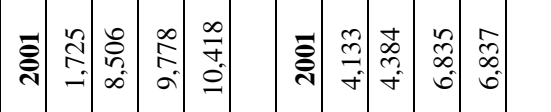

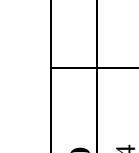

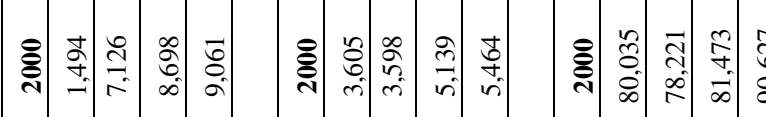

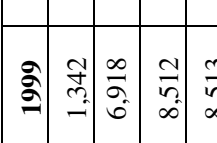

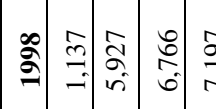

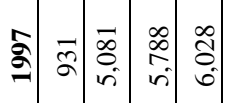

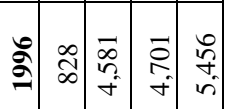

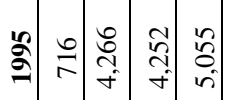

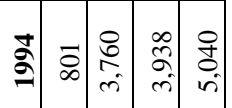

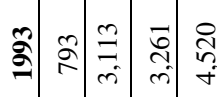

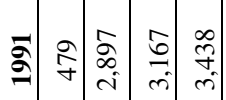

흥흄

흘

들

0
0
0
0

धि

๖ํํㅇ ఠ

图

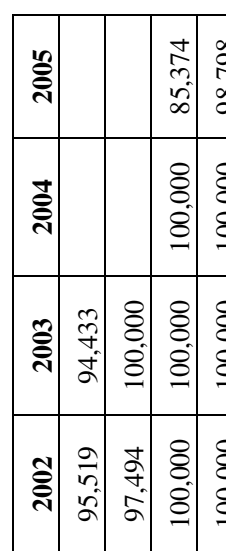

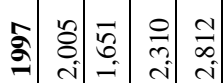

ํำ:

2 कृ

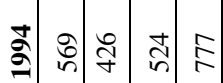

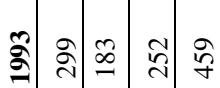

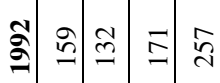

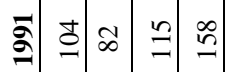

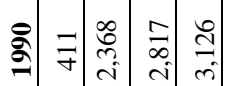

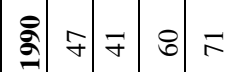

के की के के

ڤิ)

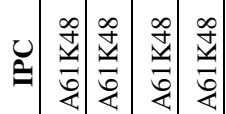

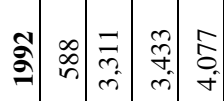

워ำ

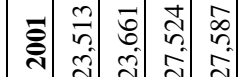

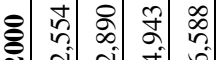

तี तิ ते तै

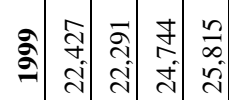

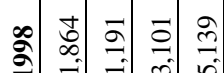

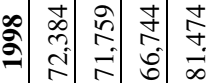

ڤำ

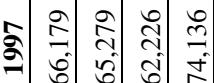

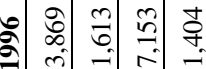

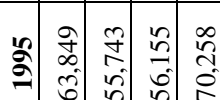

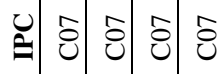

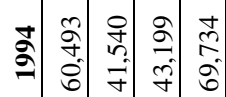

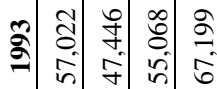

จิ

จิ

要

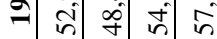

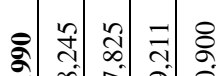

을 훙

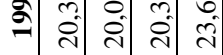

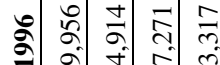

ำ

$\Rightarrow=\bar{\sim}=0$

z)

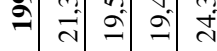

2000

$\therefore= \pm \sqrt{2}$

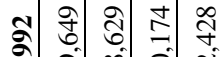

$\Rightarrow \cong \infty$ त

పิ

-2 은

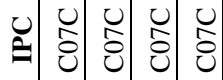

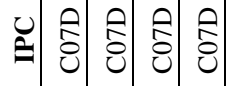

$\therefore \frac{2}{2} \div \frac{2}{2} \div$

में तं तु ते

放

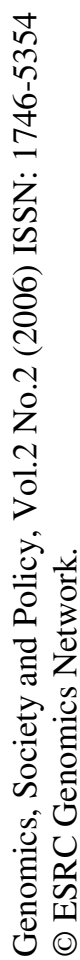

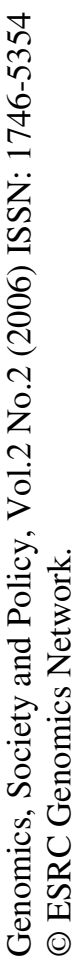

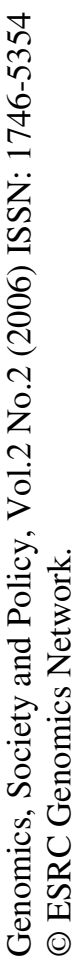

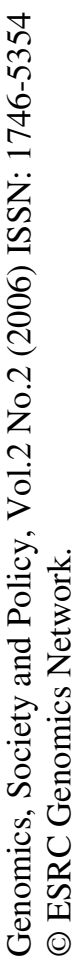

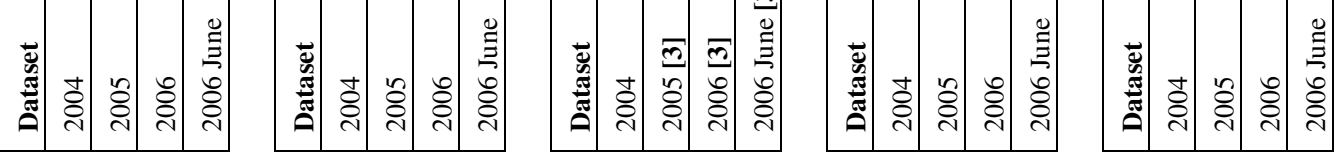




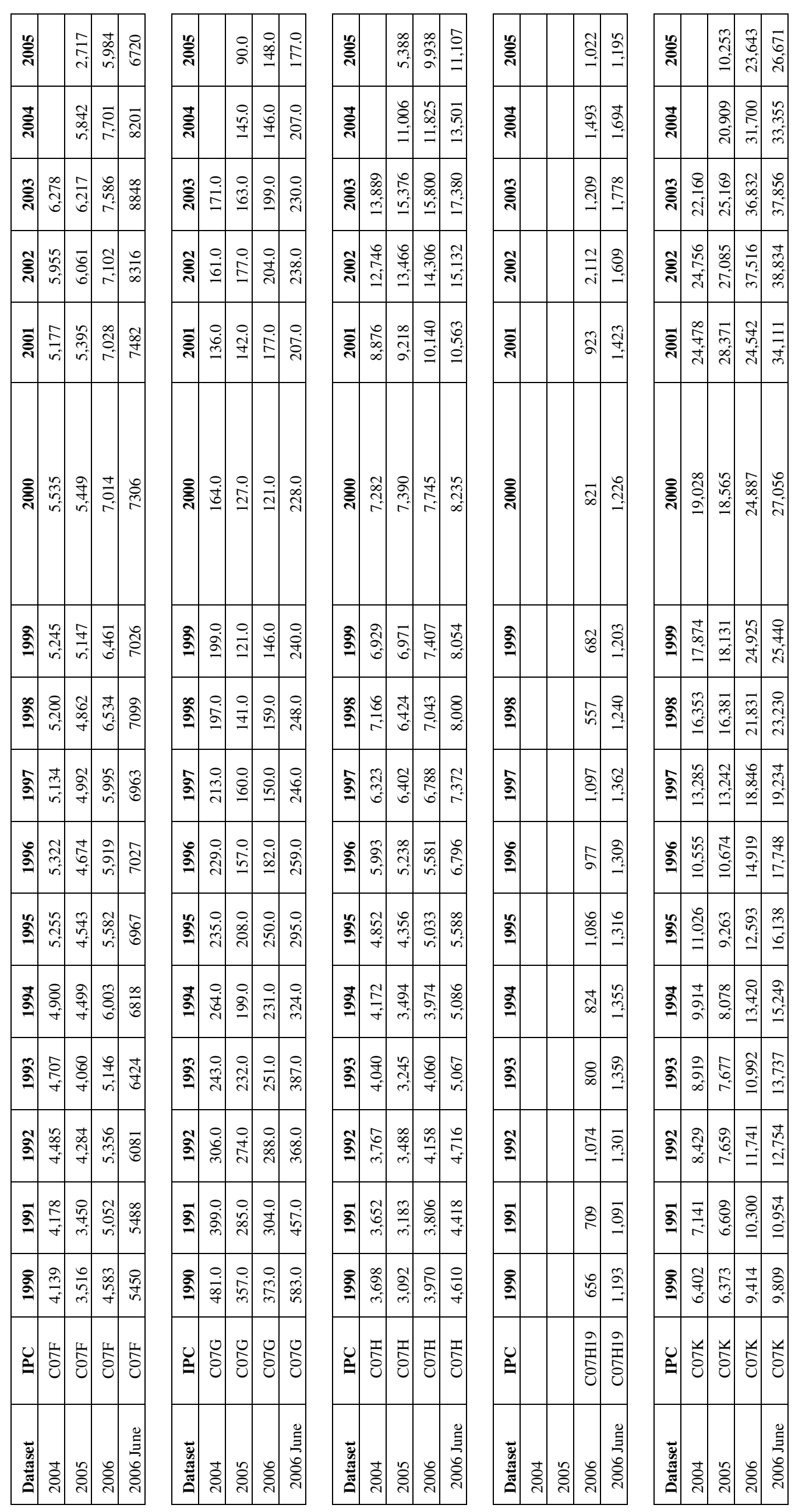




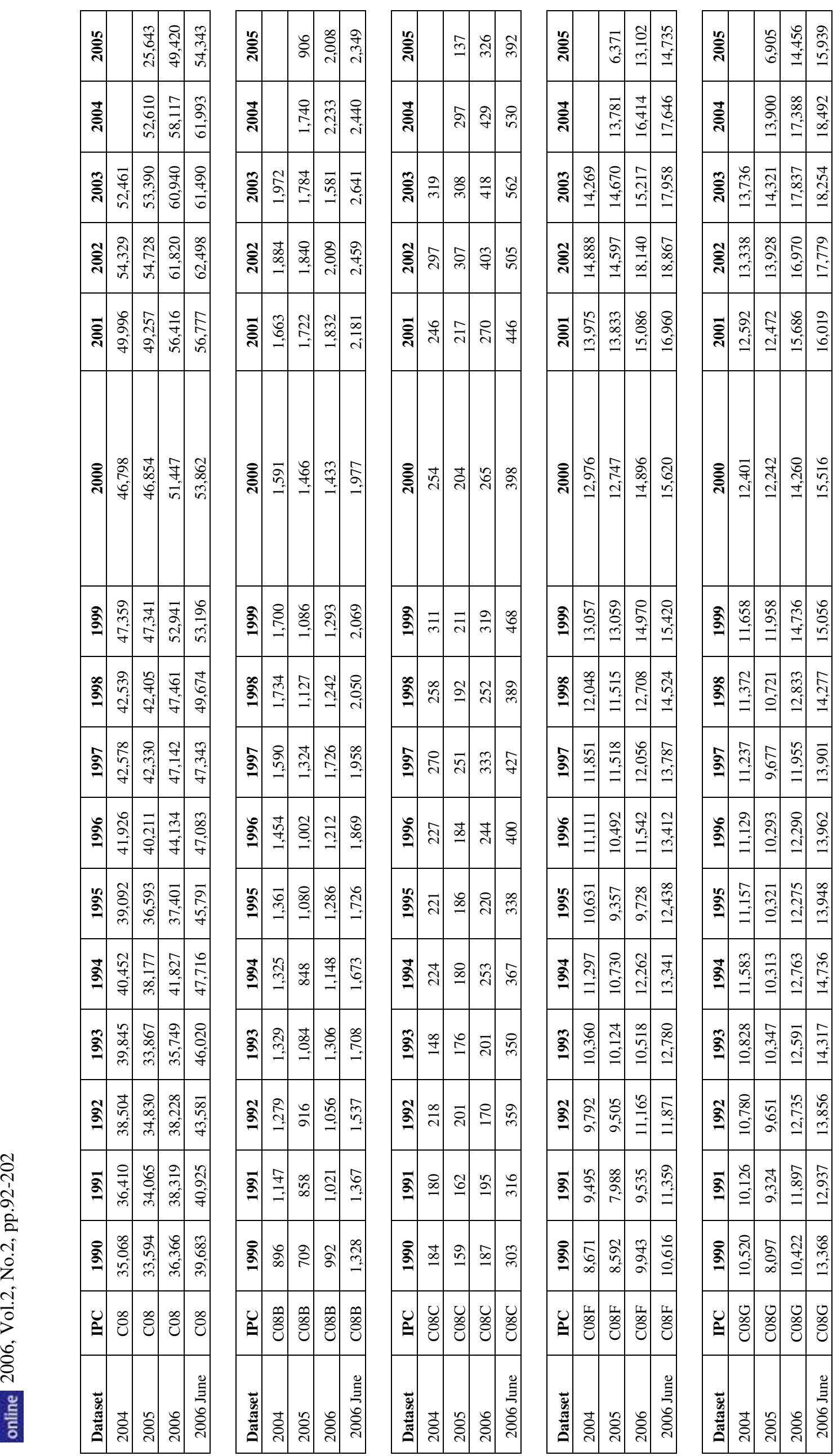

ํํㅇㅈㅗ

ป ส

ह

包

क त

धी

造

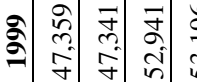

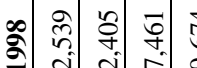

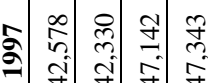

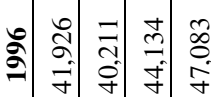

ڤิ)

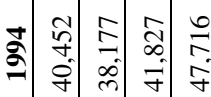

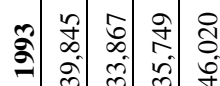

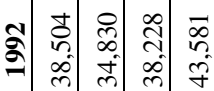

i $\quad$ :

i

$0 \infty$ 


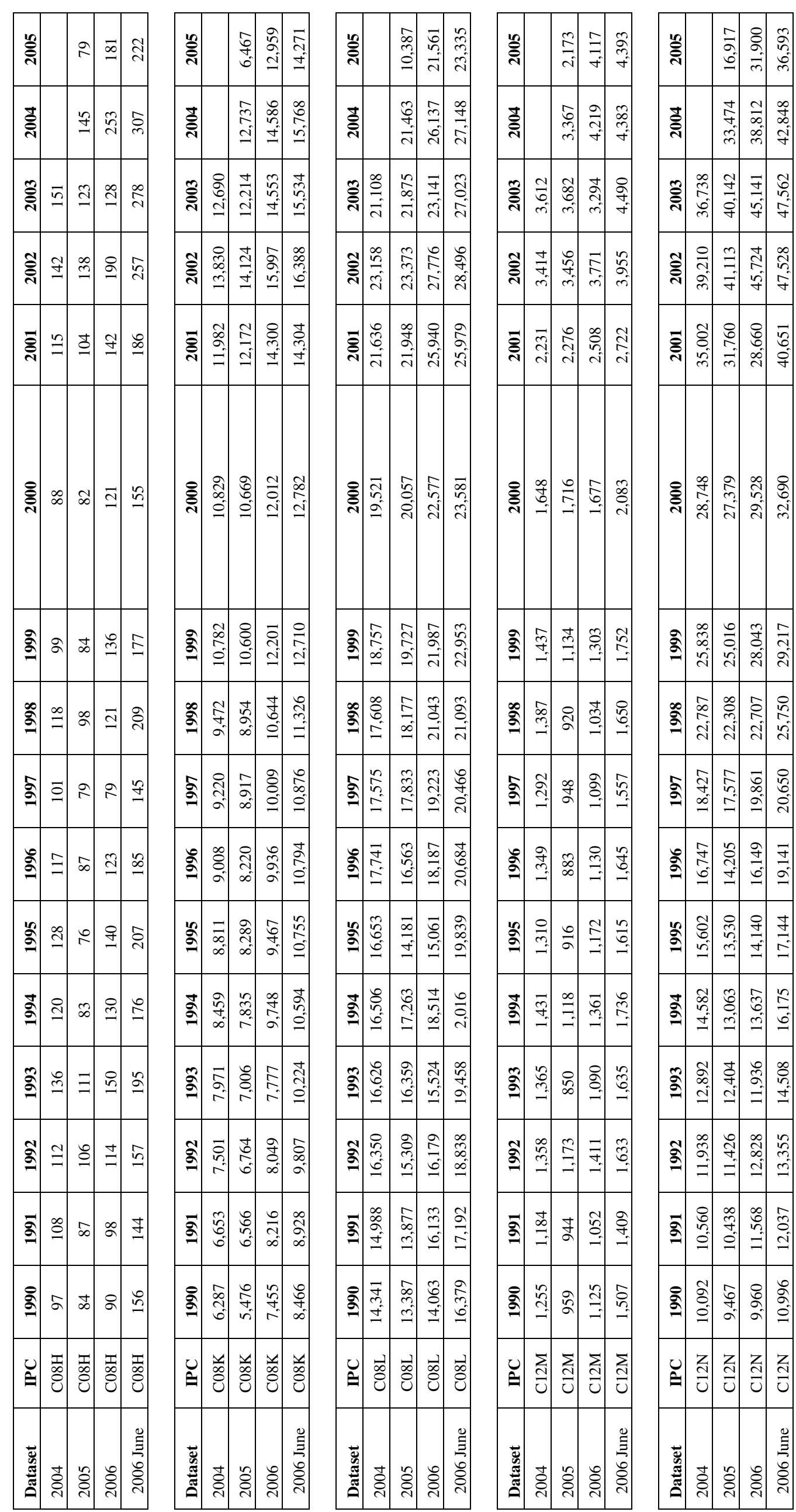




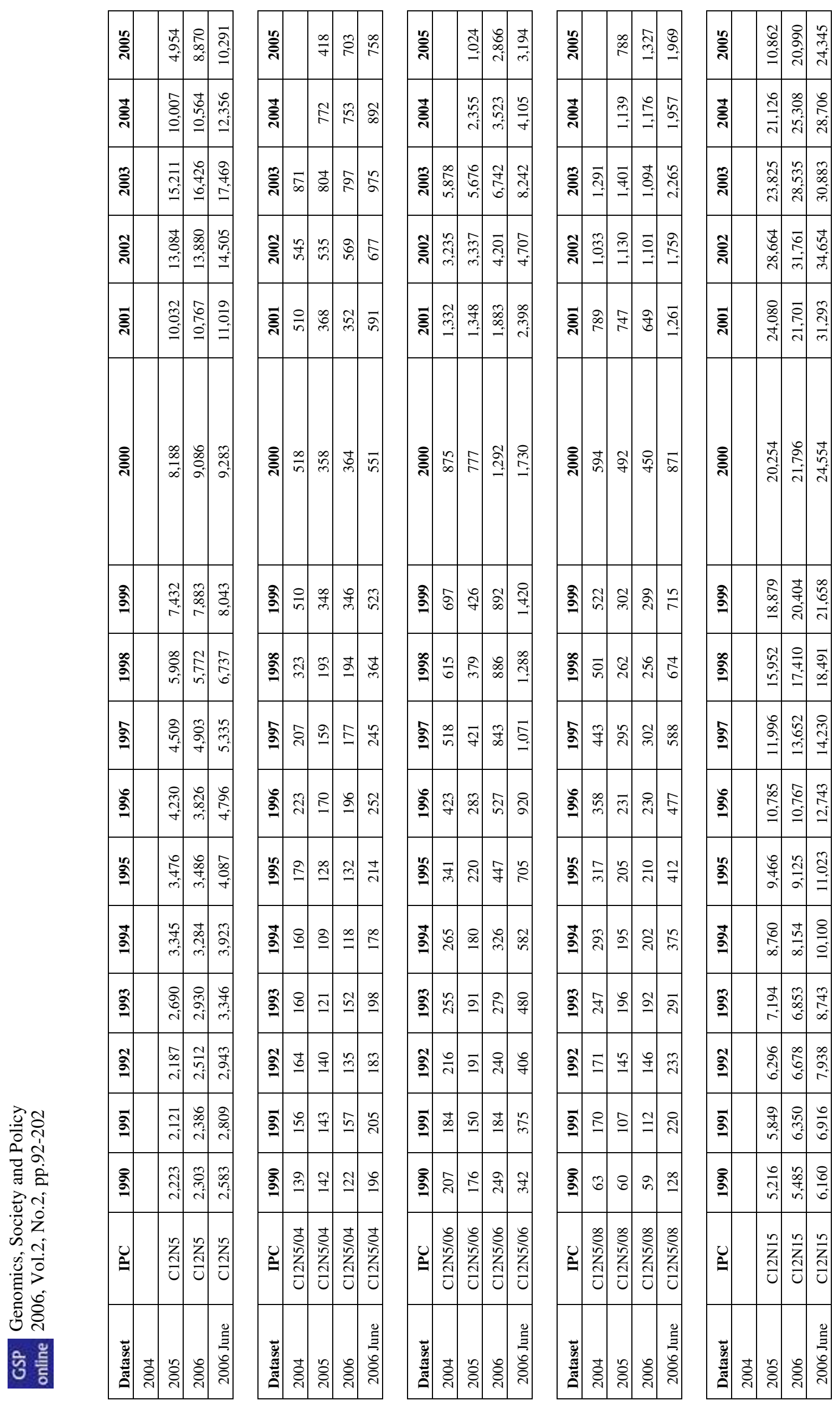




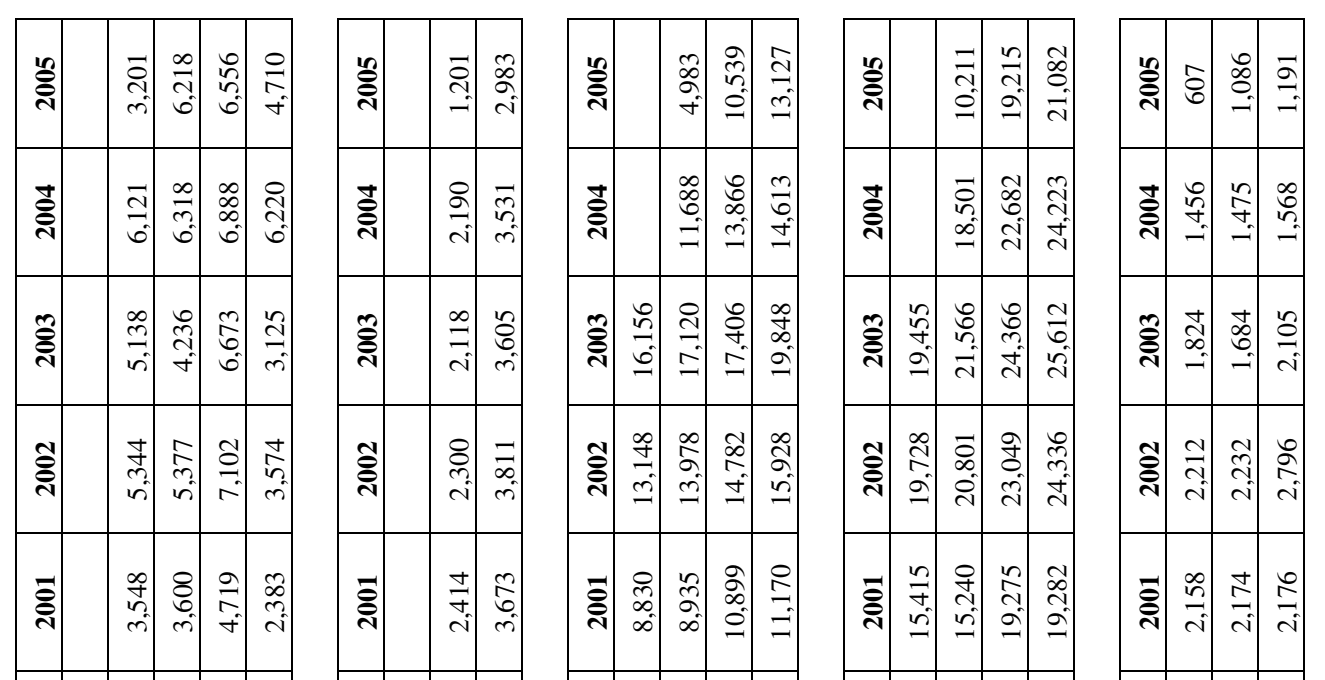

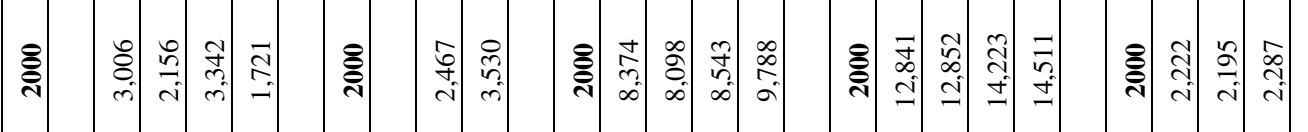

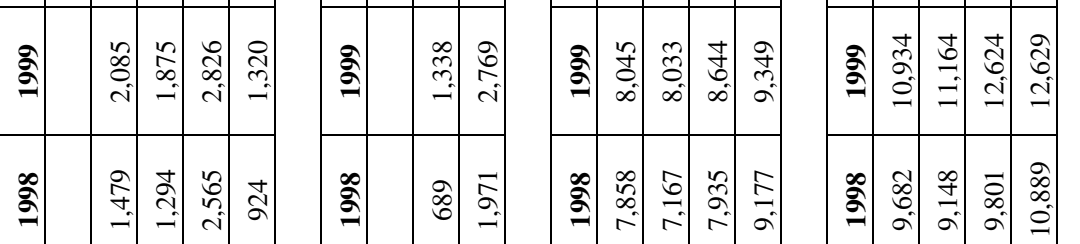

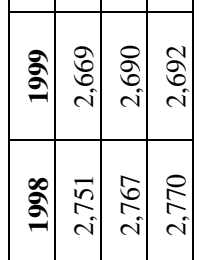

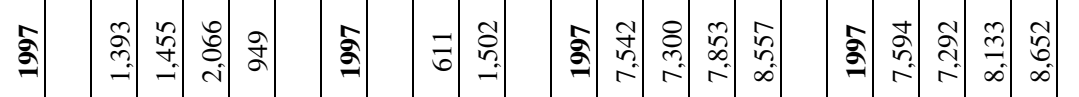

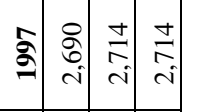

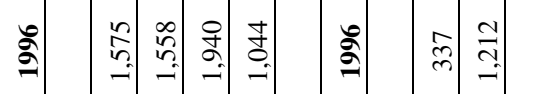

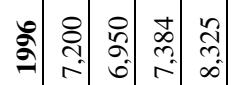

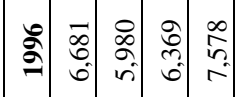

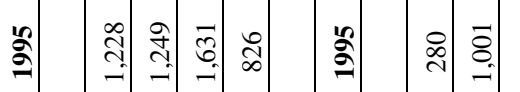

:

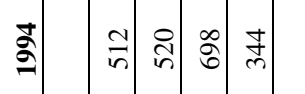

ปิ

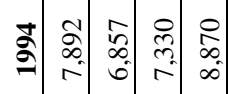

气̊

ลำำ 육

ڤ.

ปิ

ปิ

ลิ)

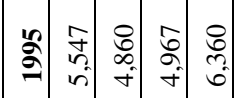

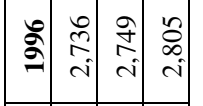

हิ)

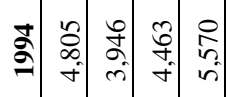

ปิ)

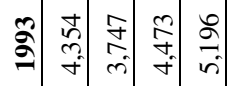

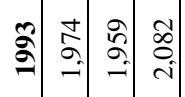

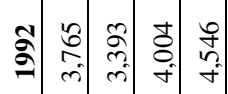

ปี ปี

चิ

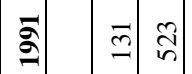

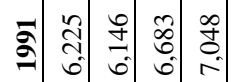

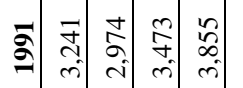

ڤิ)

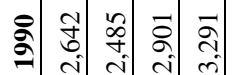

흘

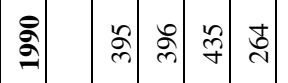

के के ले

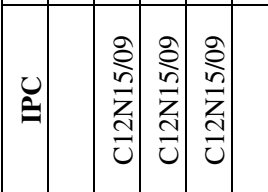

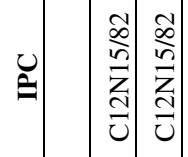

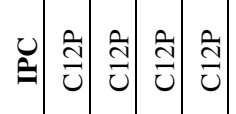

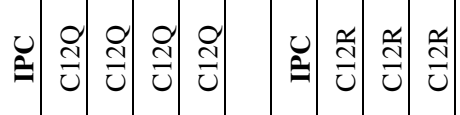

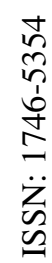

है

กั

ก)

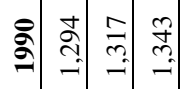

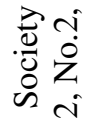

हैं

造 


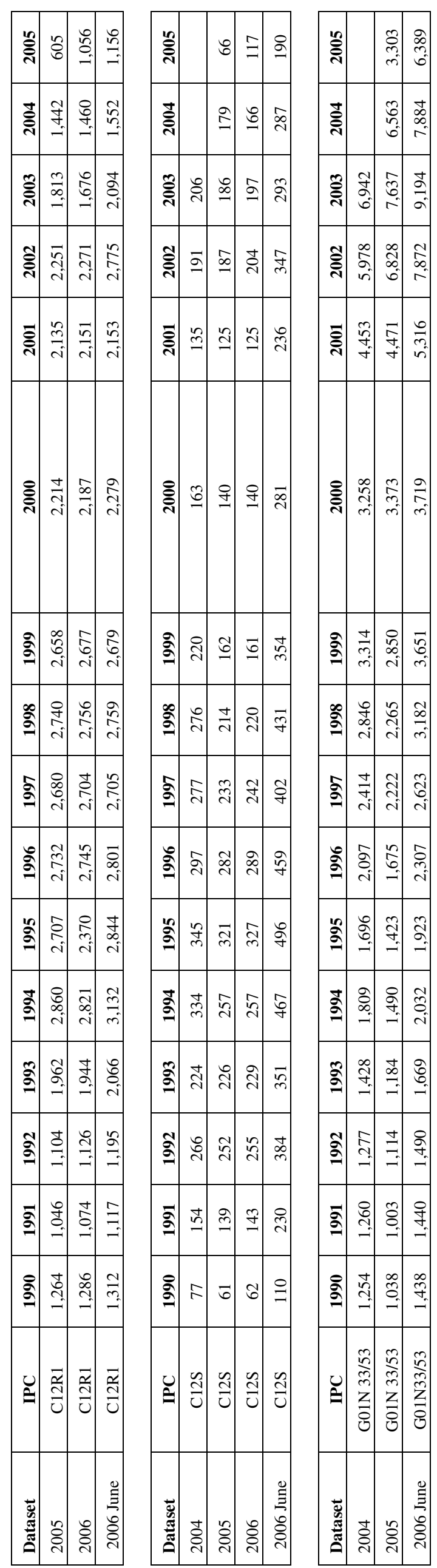

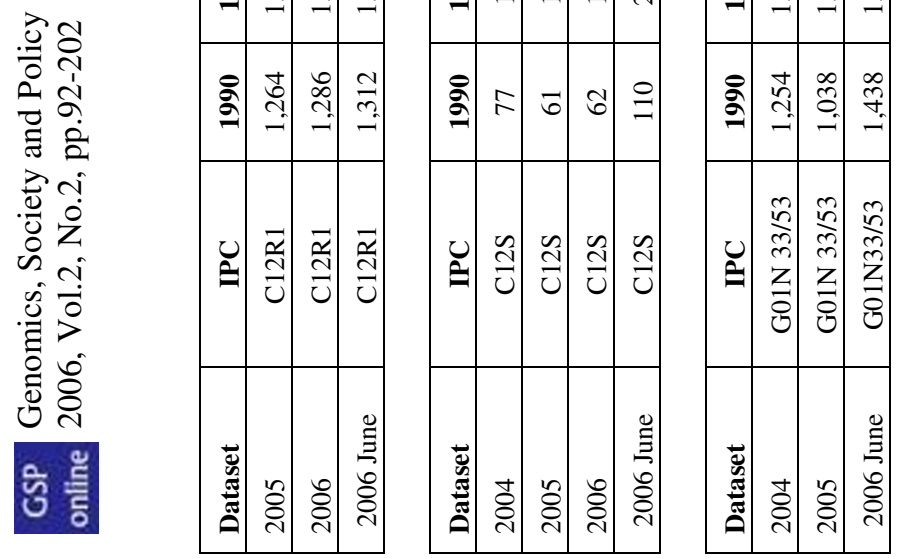


GSP Genomics, Society and Policy online 2006, Vol.2, No.2, pp.92-202

The following graphs provide basic comparisons of the results of searches of esp@cenet for a number of the main indicators (see notes above)

Figure 1

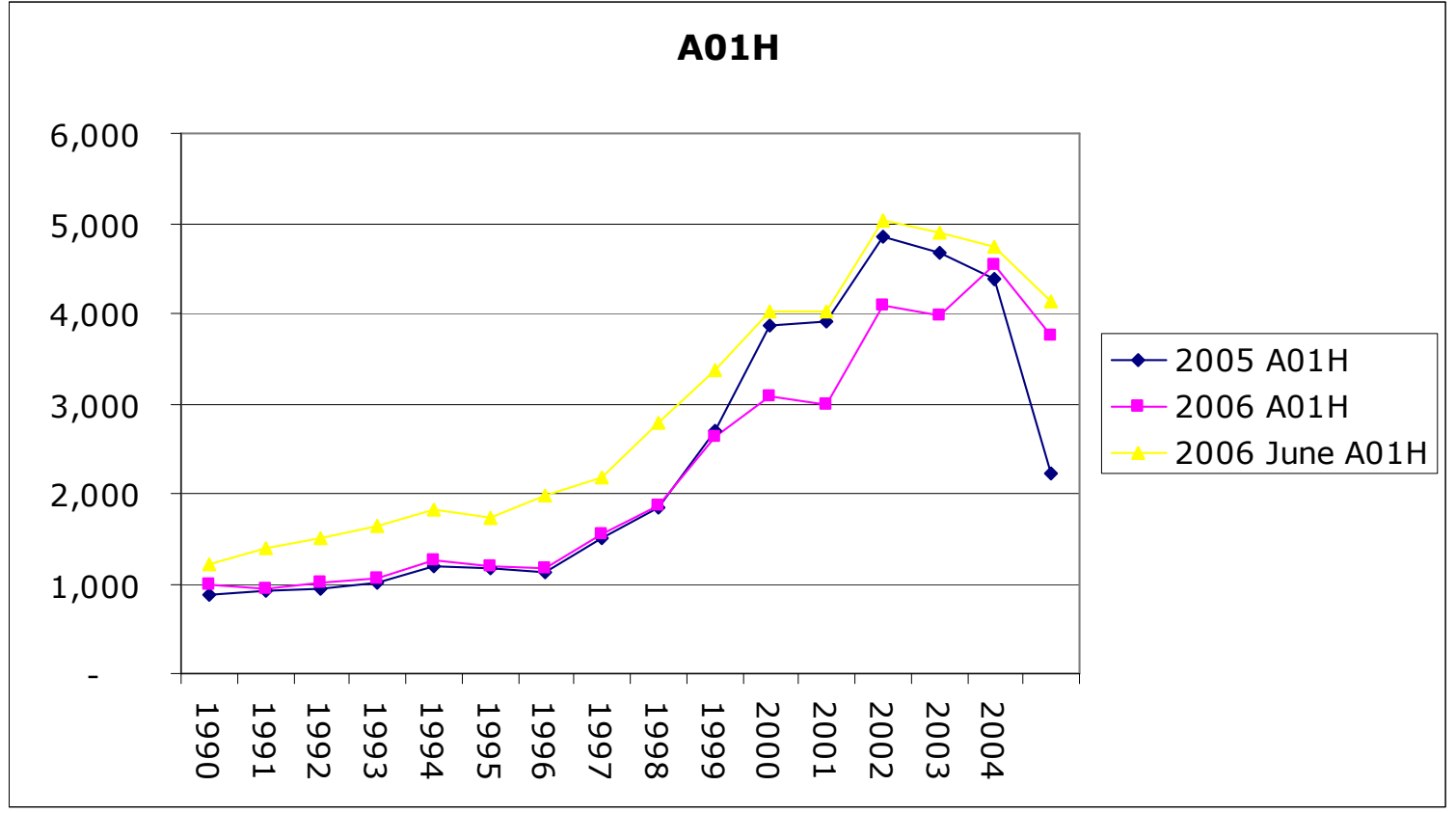

Figure 2

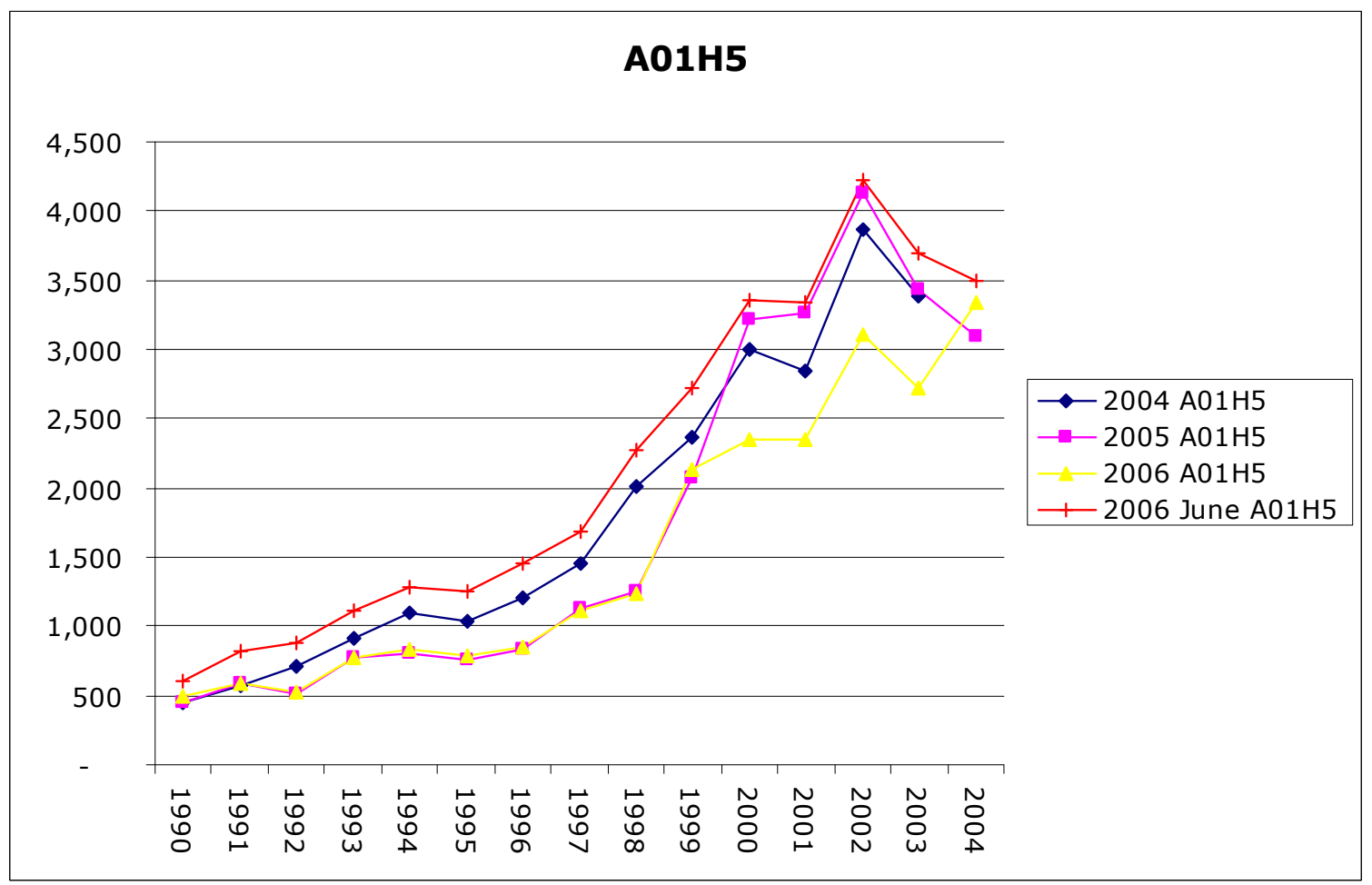


Figure 3

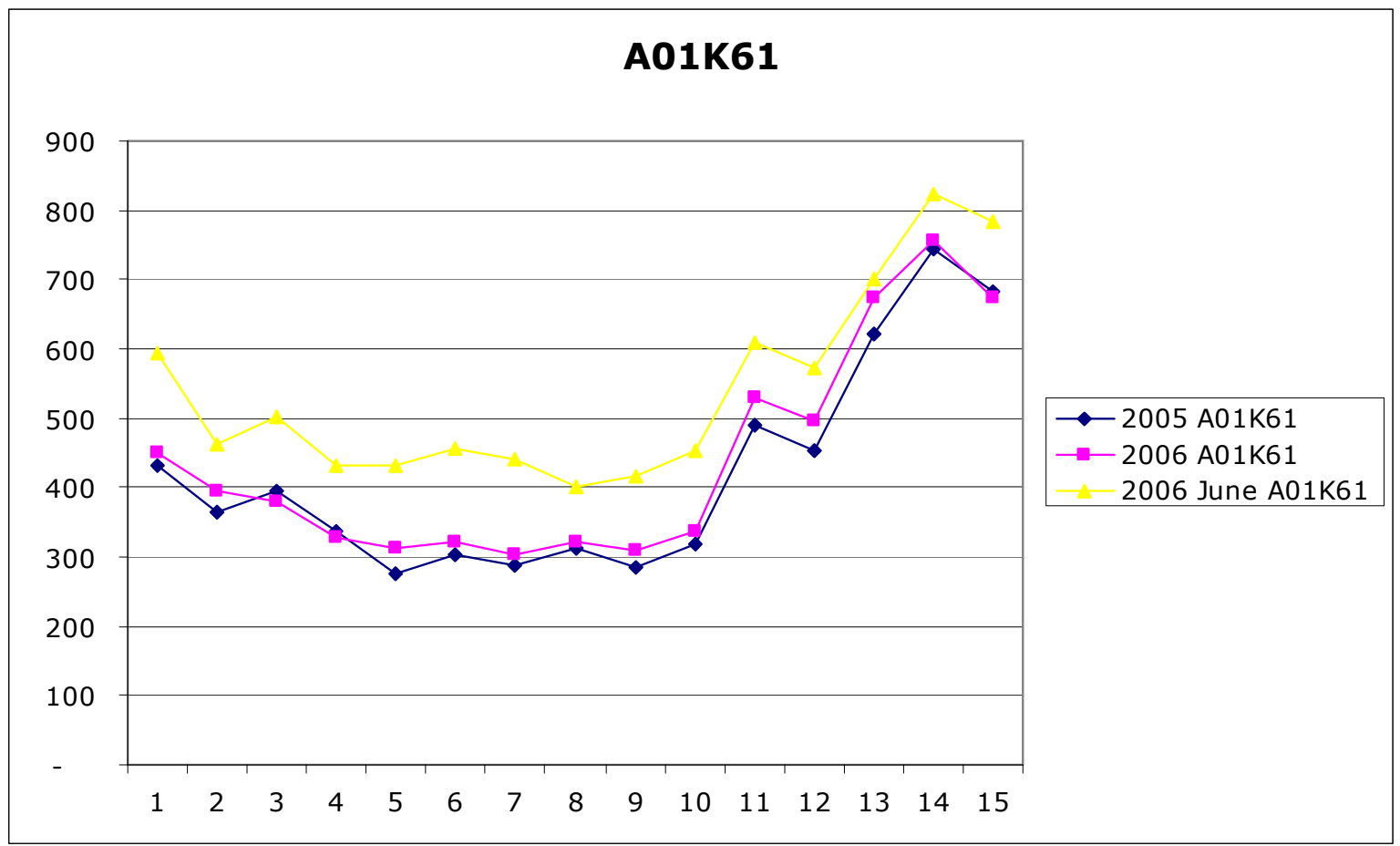

\section{Figure 4}

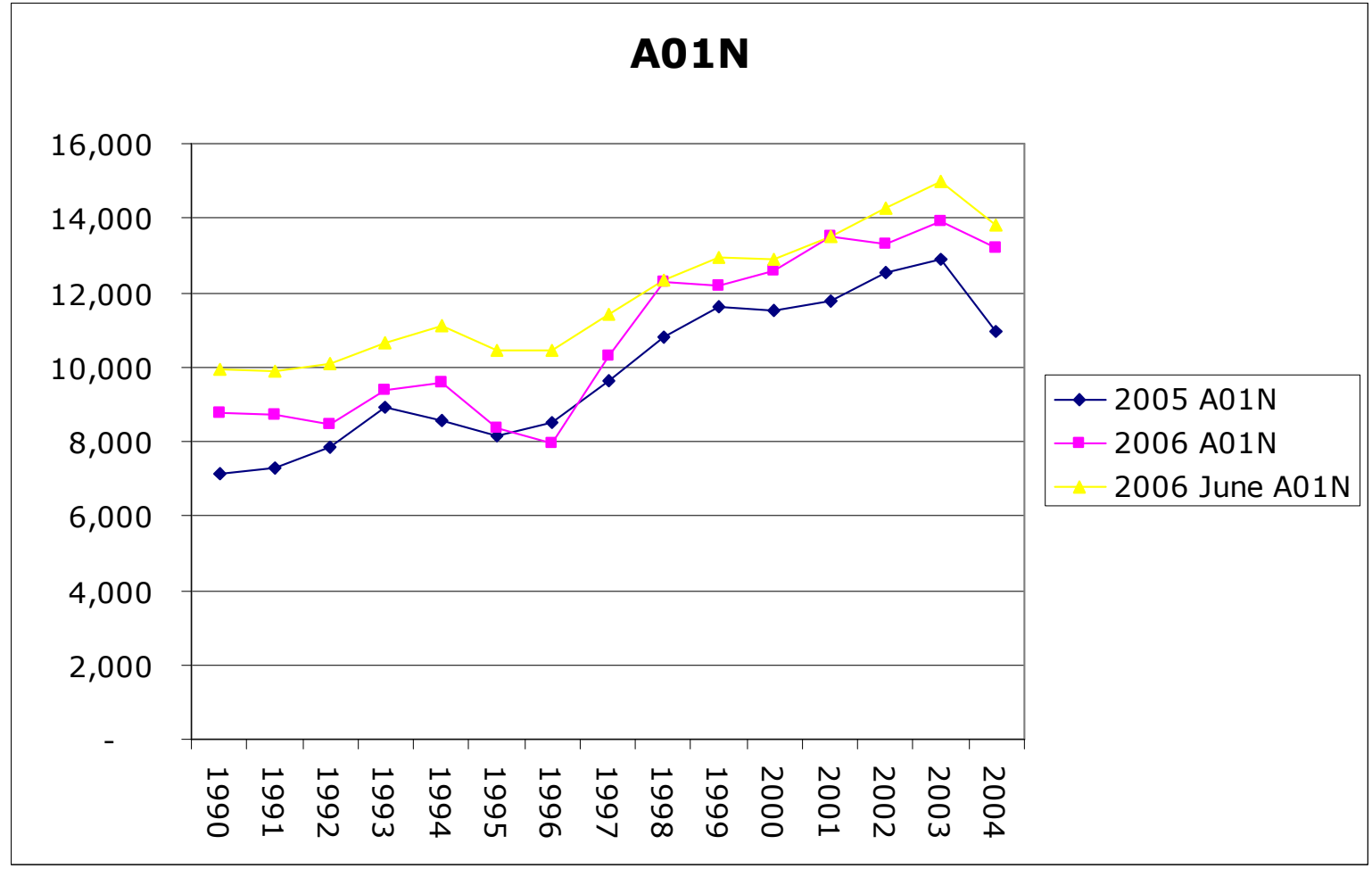


Figure 5

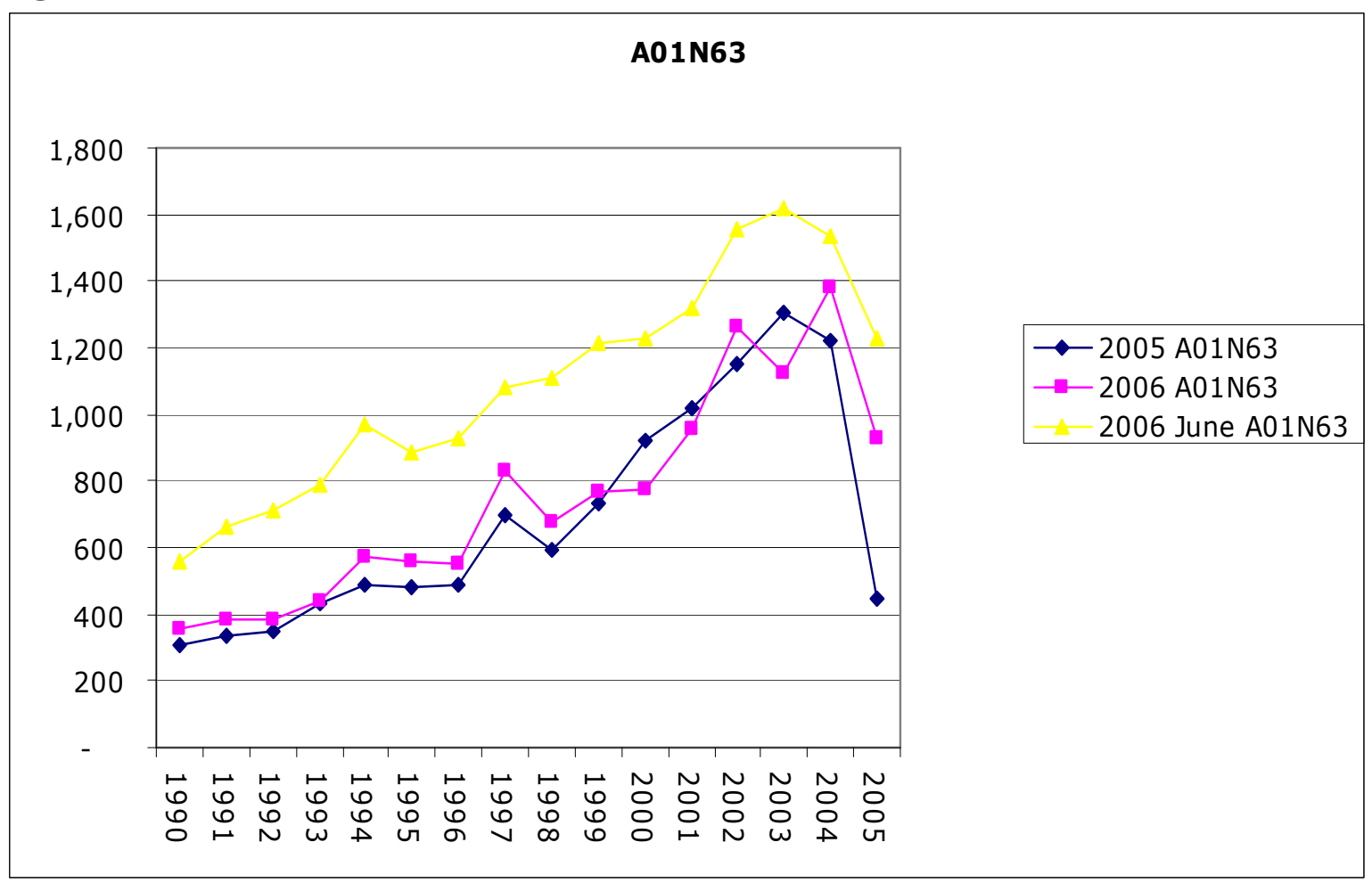

Figure 6

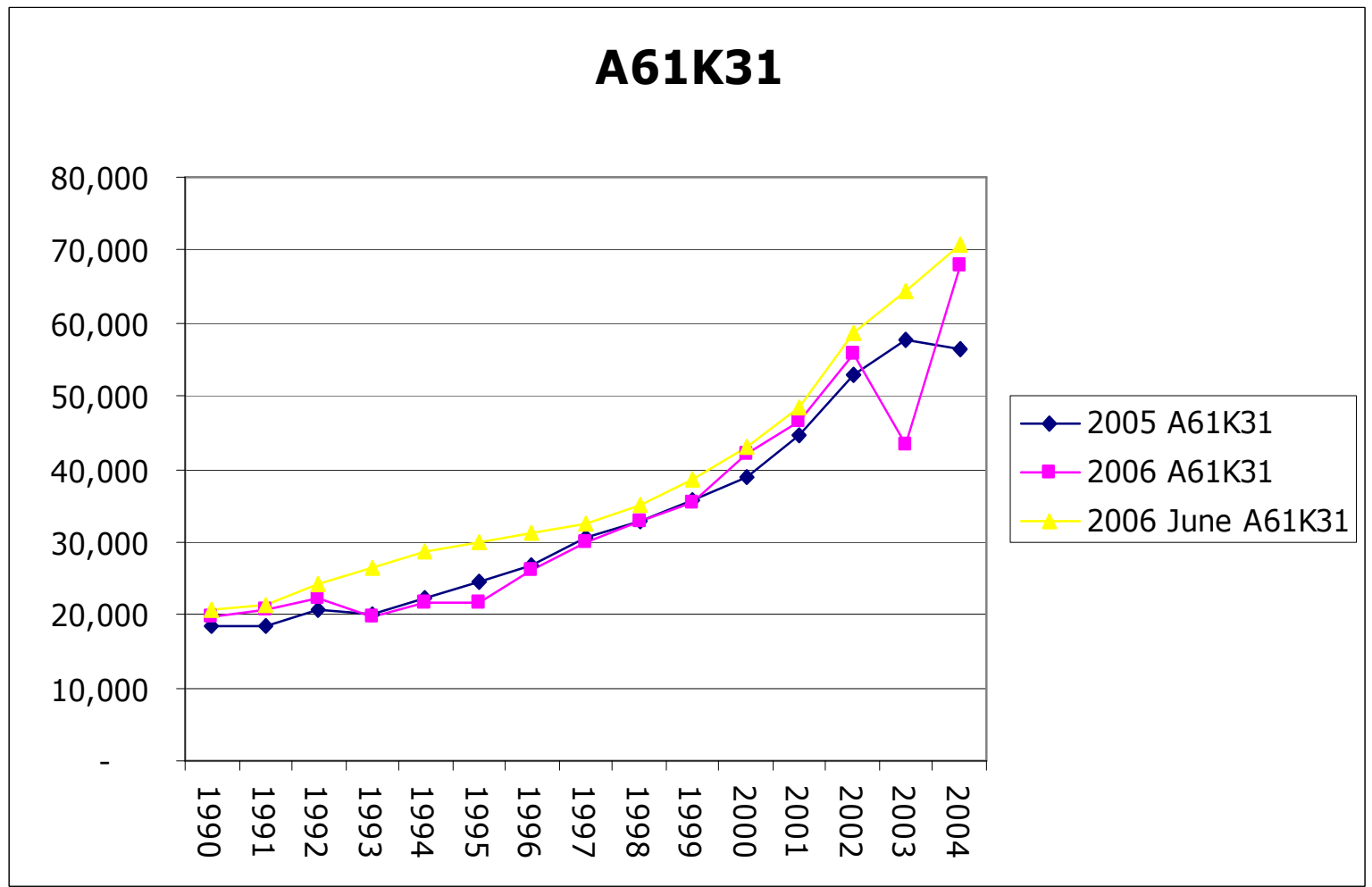


GSP Genomics, Society and Policy

Figure 7

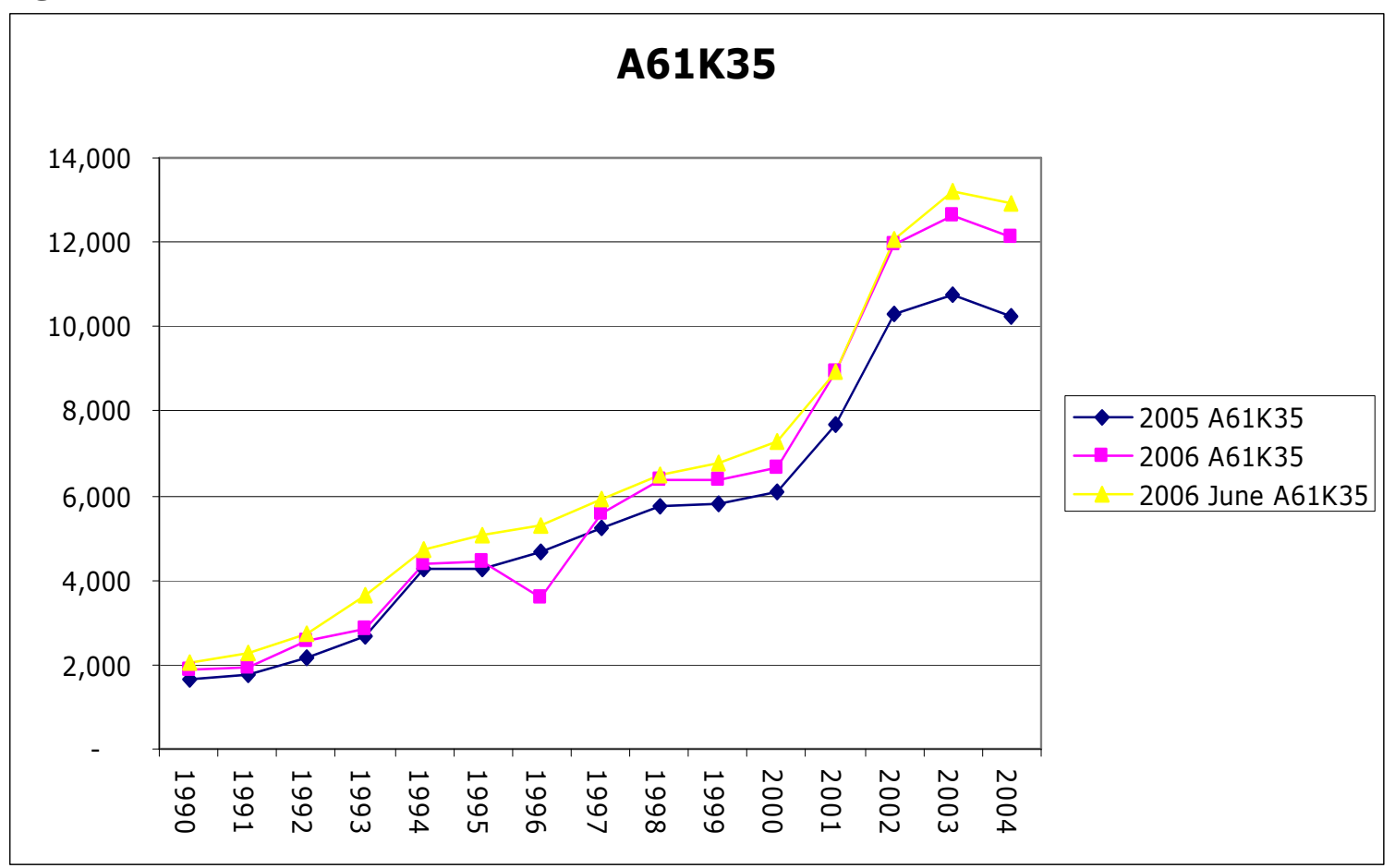

\section{Figure 8}

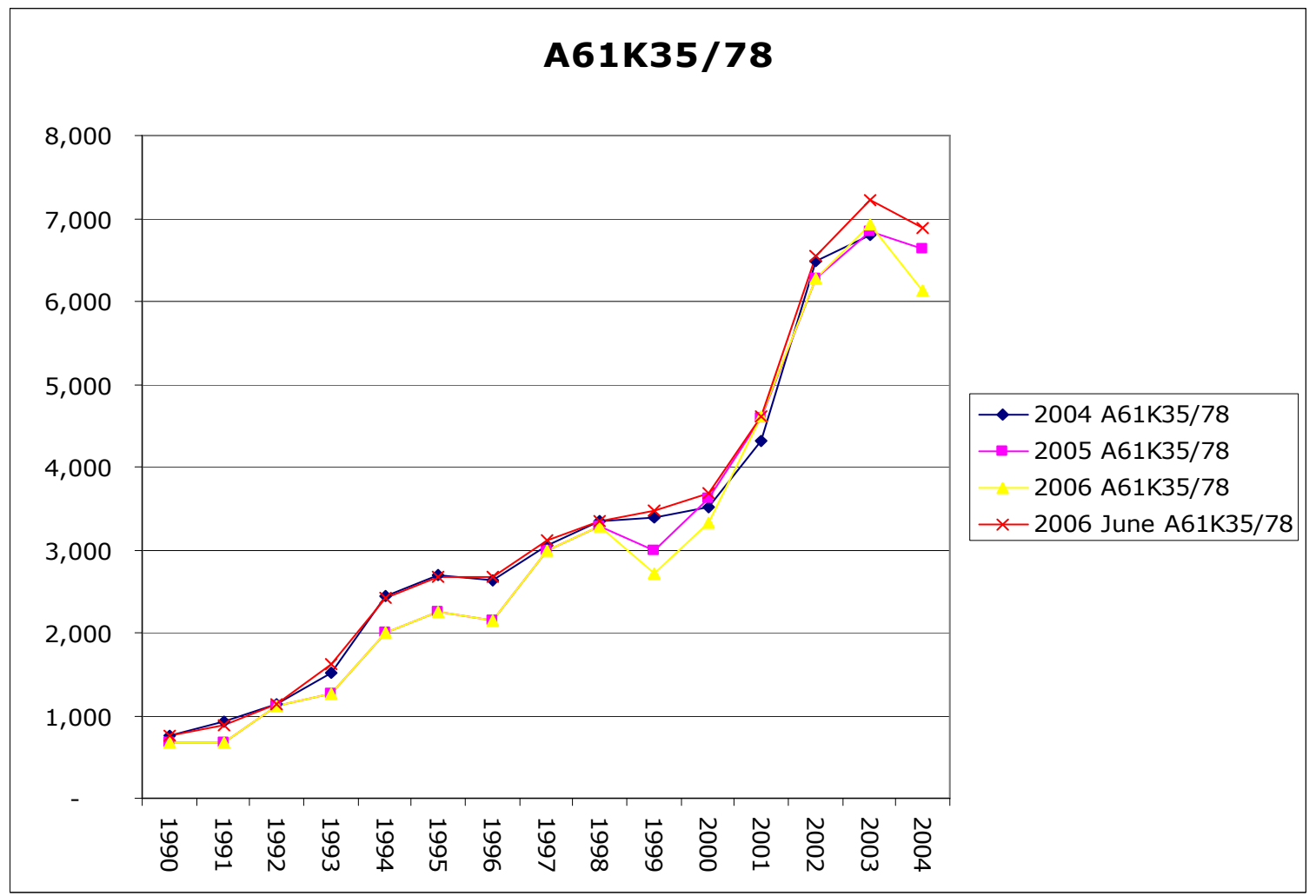


Figure 9 - Medicinal preparations containing peptides

Note that the variation reflects the use of A61K38/00 in conducting searches rather than A61K38 as commonly awarded by examiners.

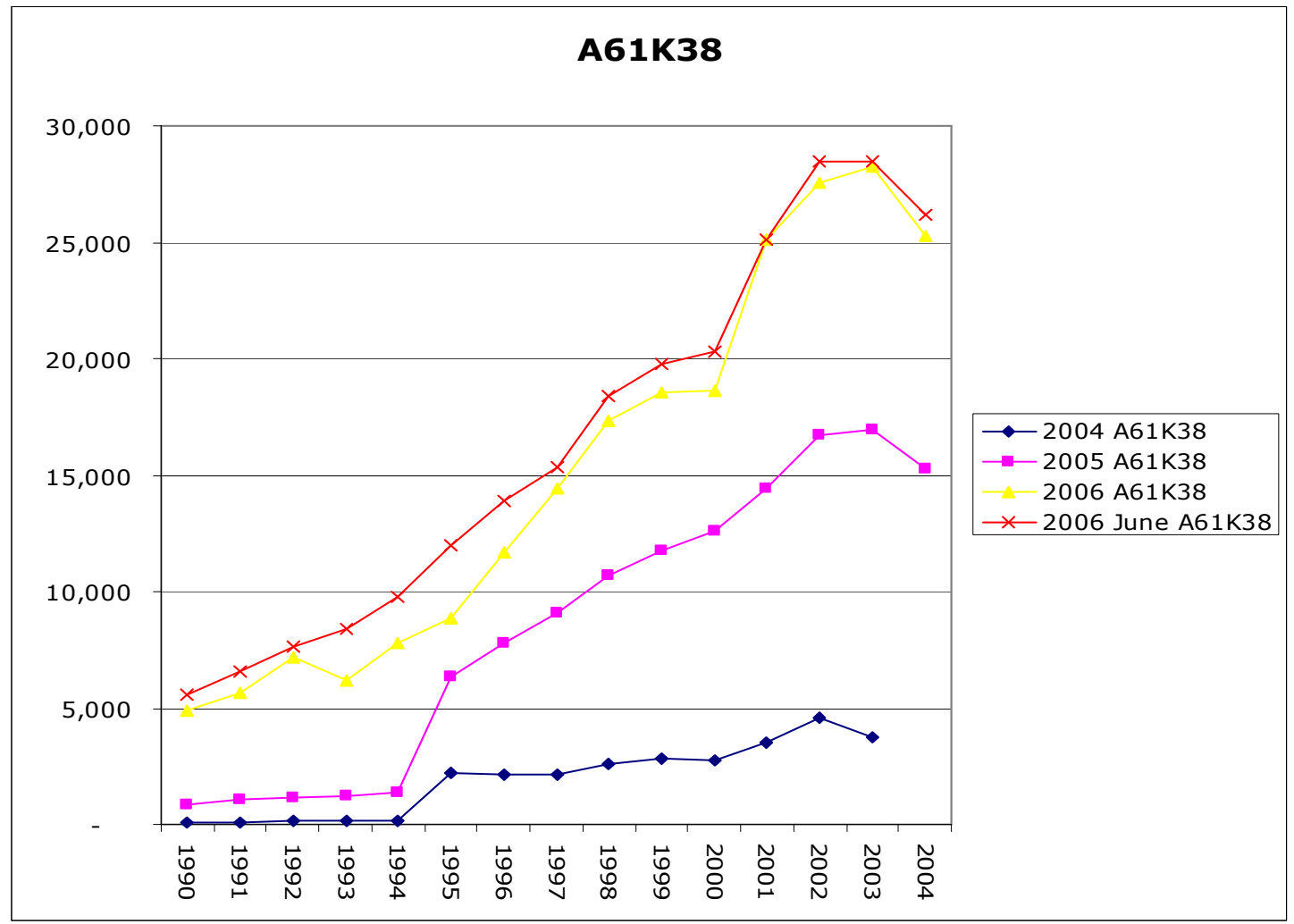

Figure 10 (see note for A61K38 in relation to the search in 2004)

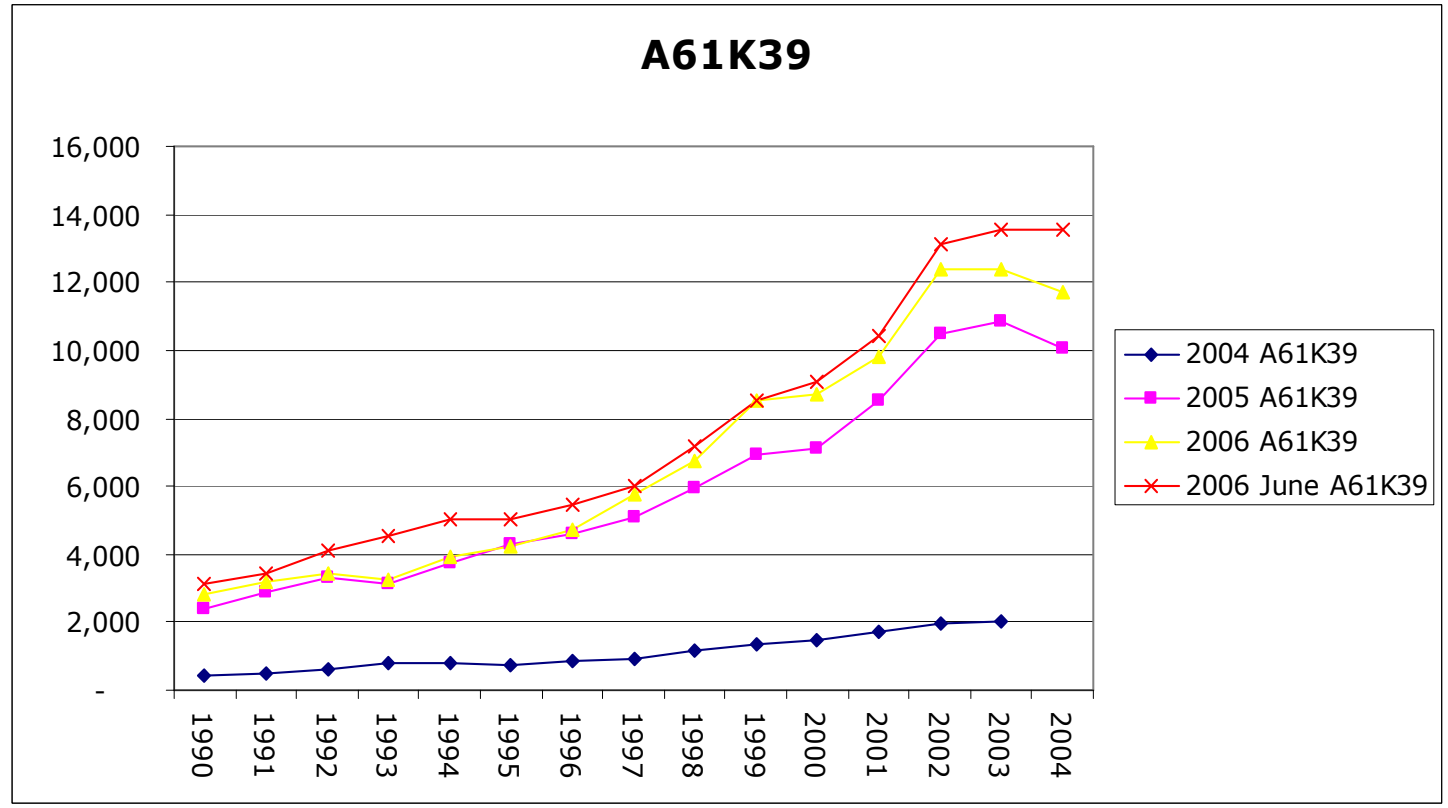


GSP Genomics, Society and Policy

Figure 11

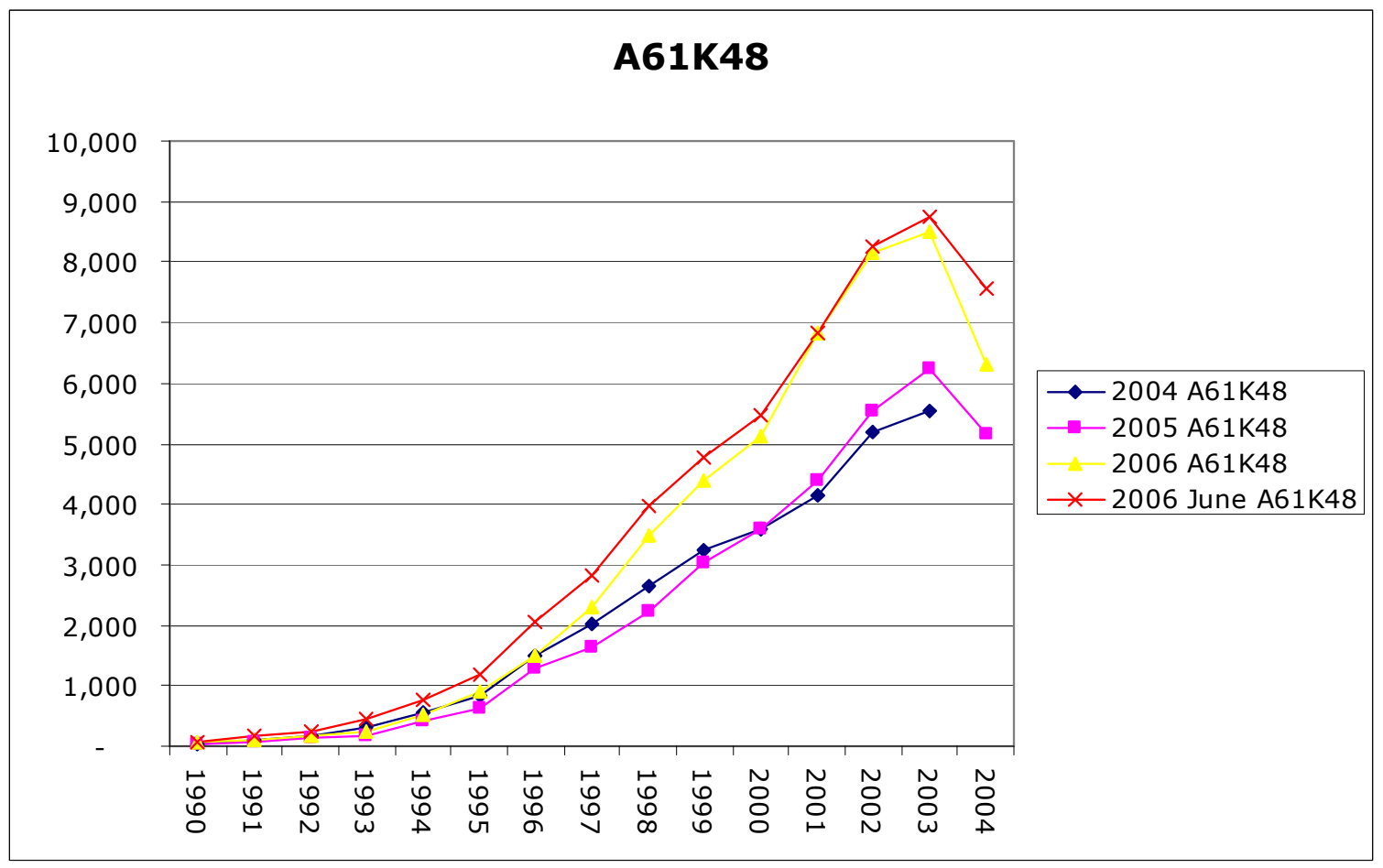

Figure 12

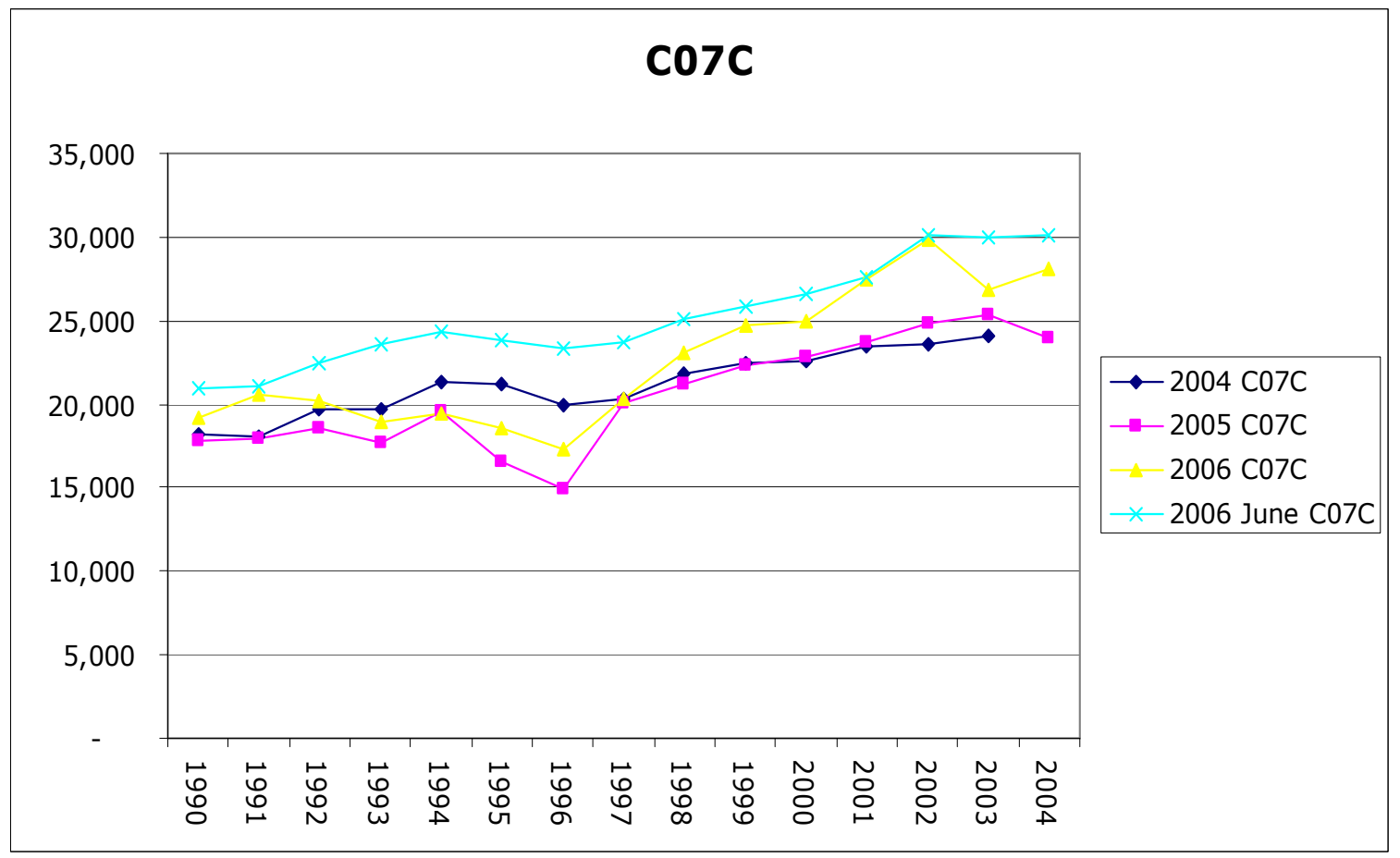


Figure 13

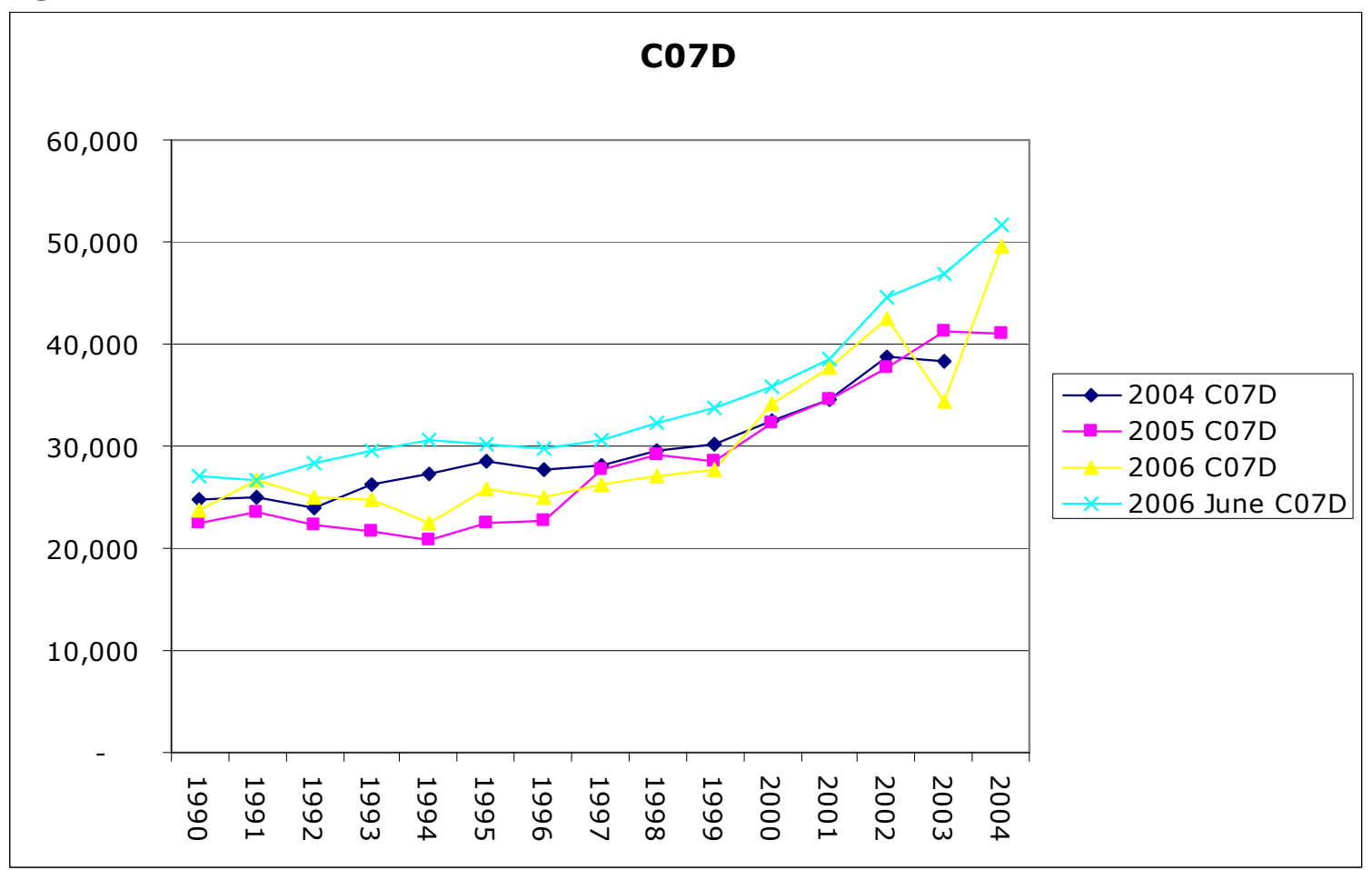

\section{Figure 14}

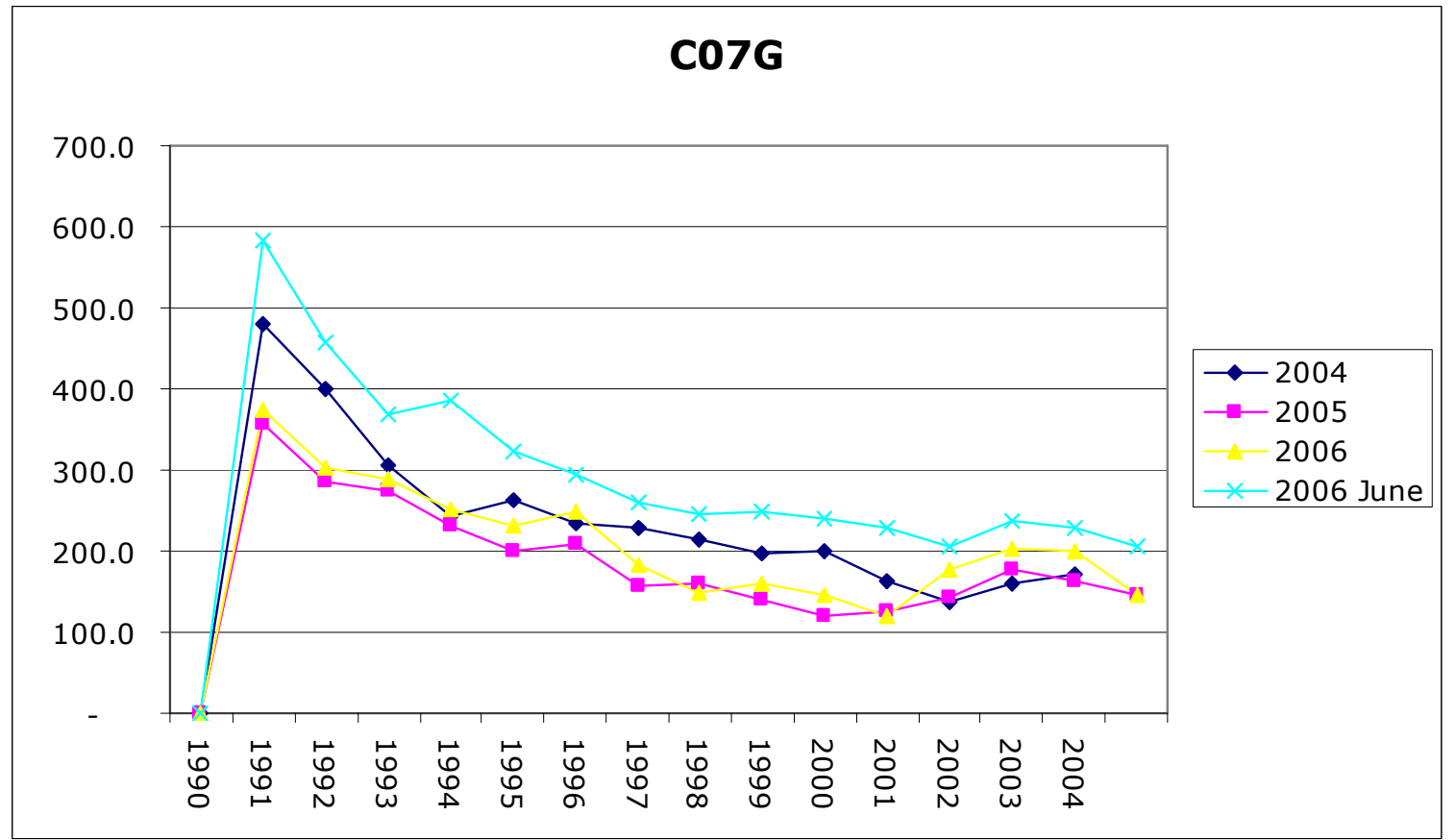


GSP Genomics, Society and Policy

Figure 15

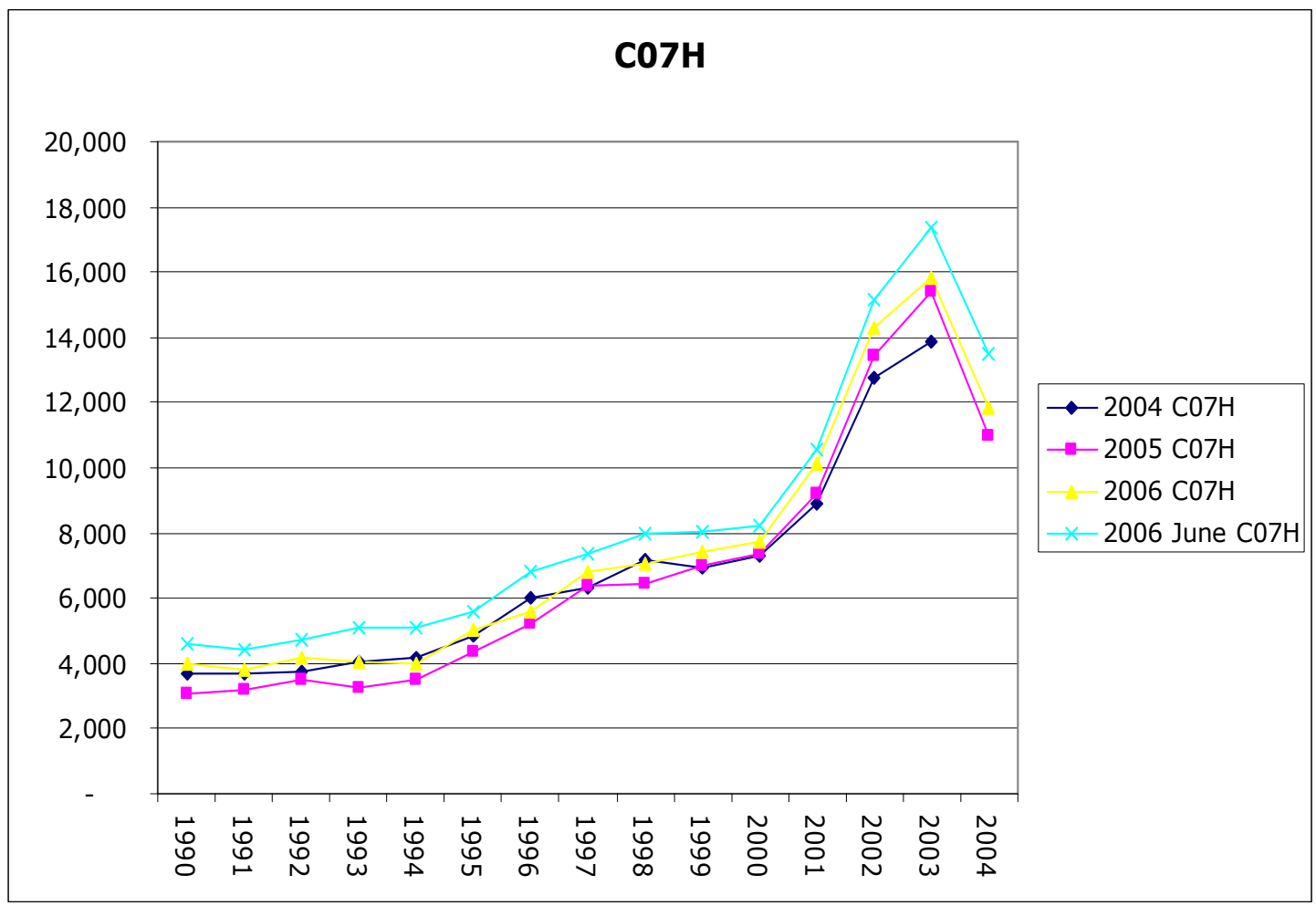

Figure 16

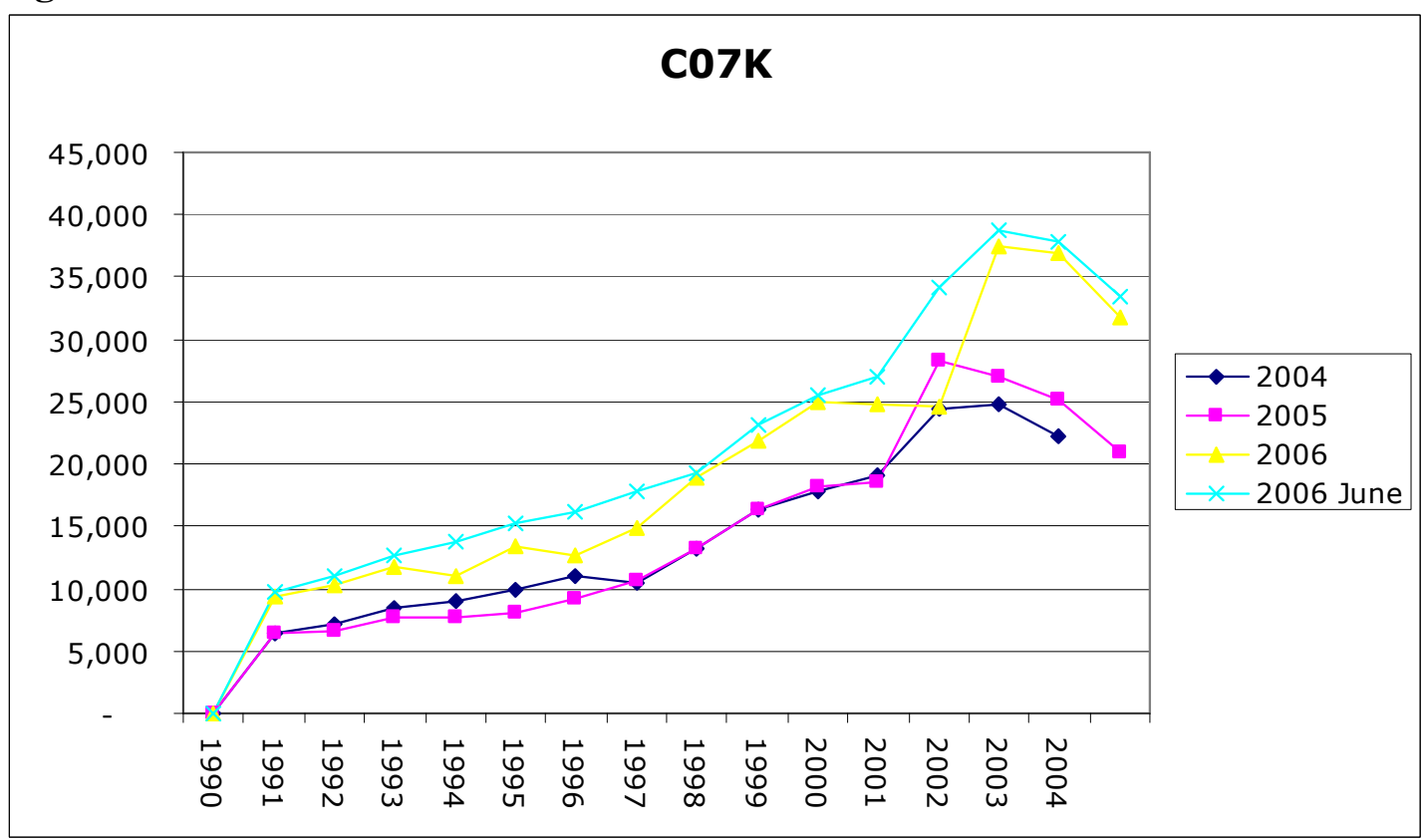


GSP Genomics, Society and Policy

Figure 17

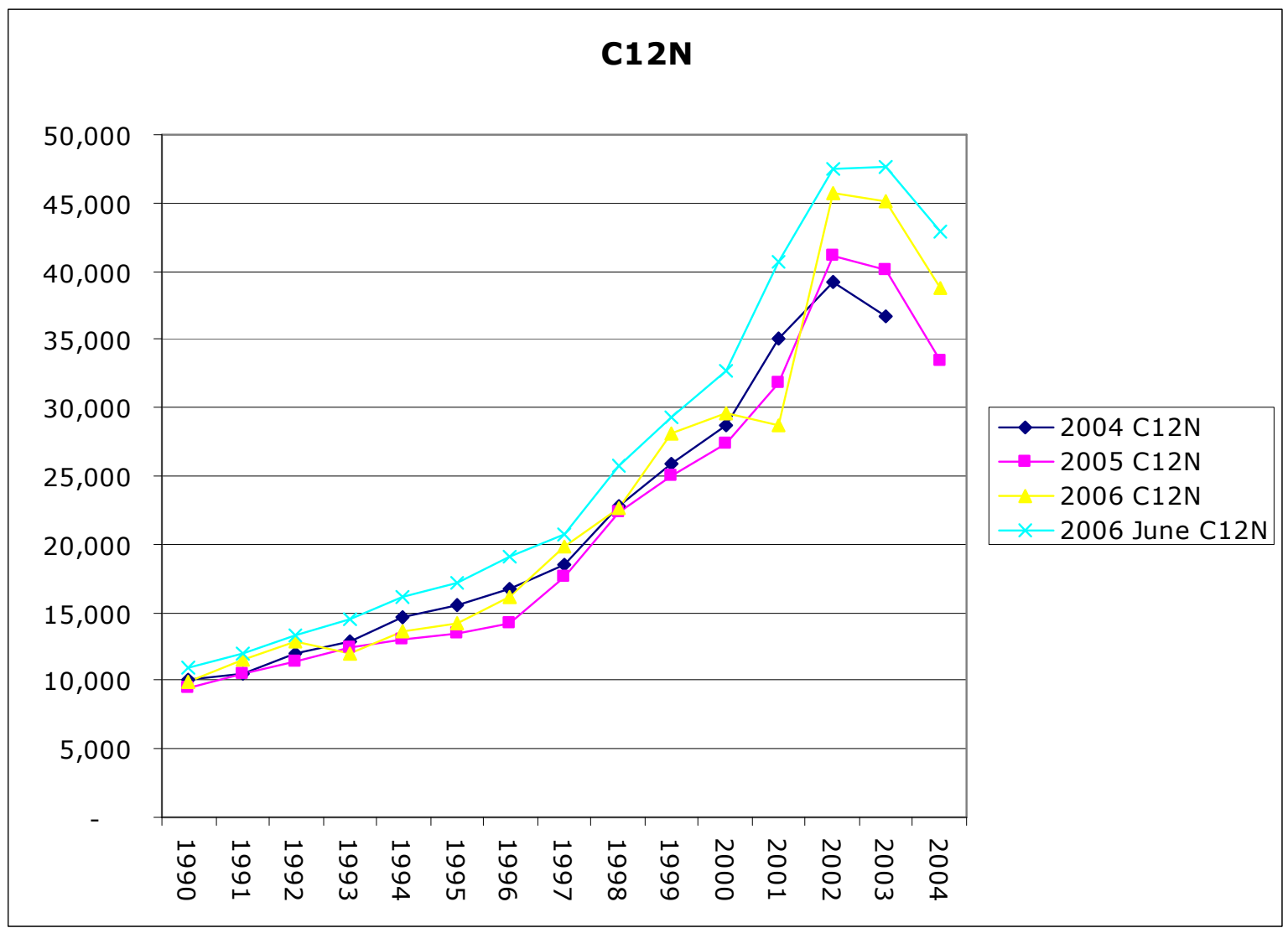

\section{Figure 18}

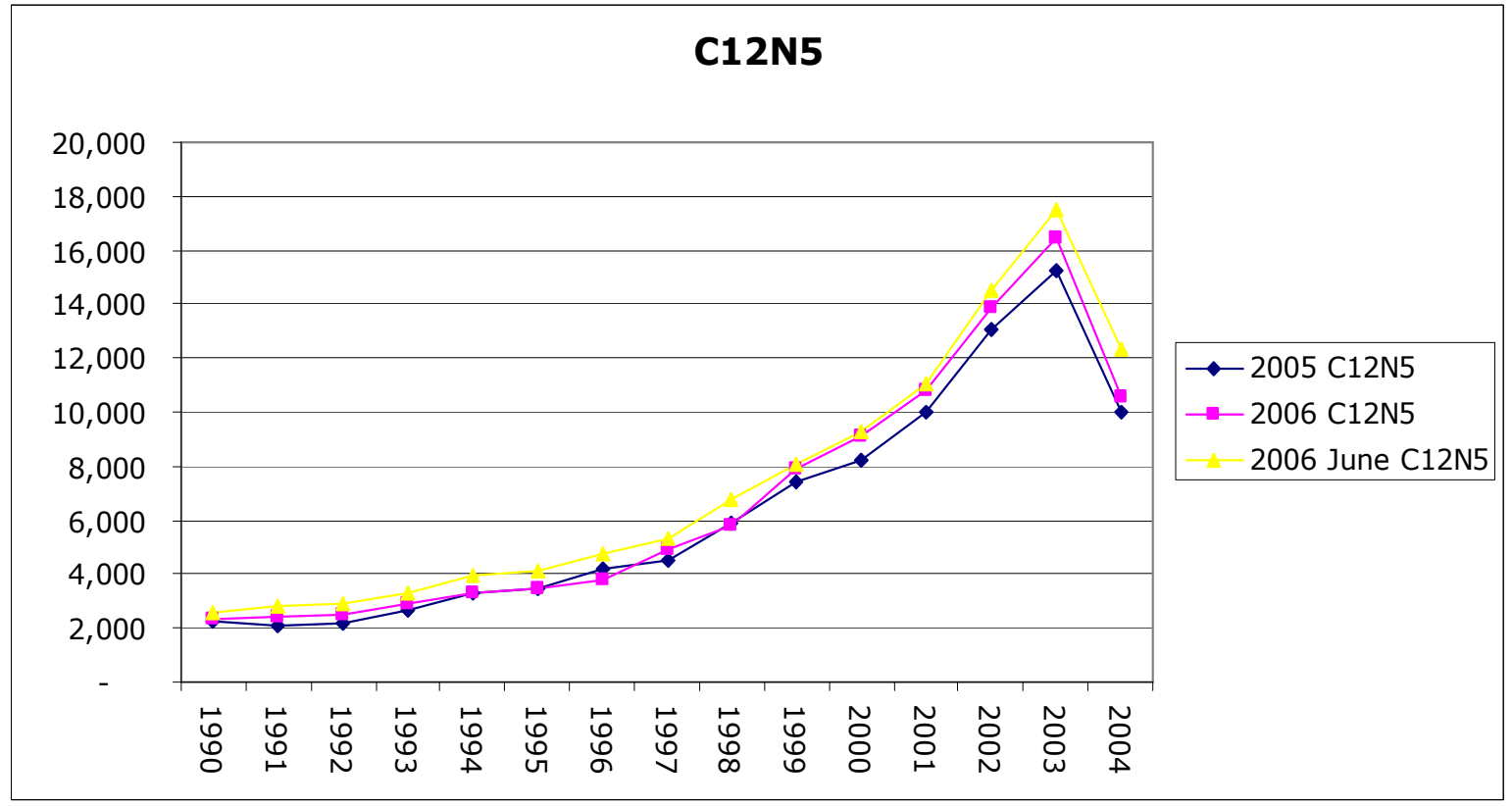


Figure 19

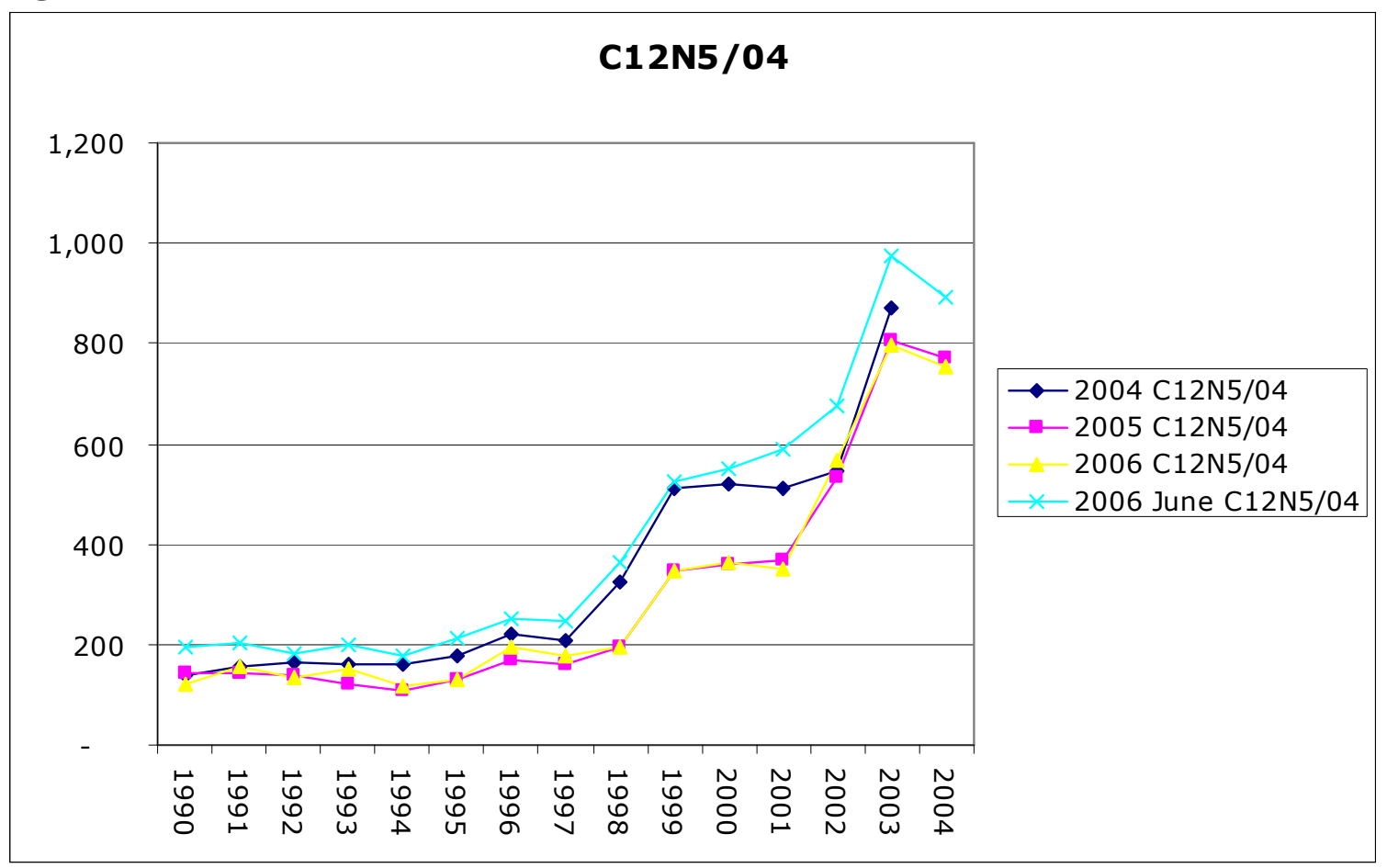

Figure 20

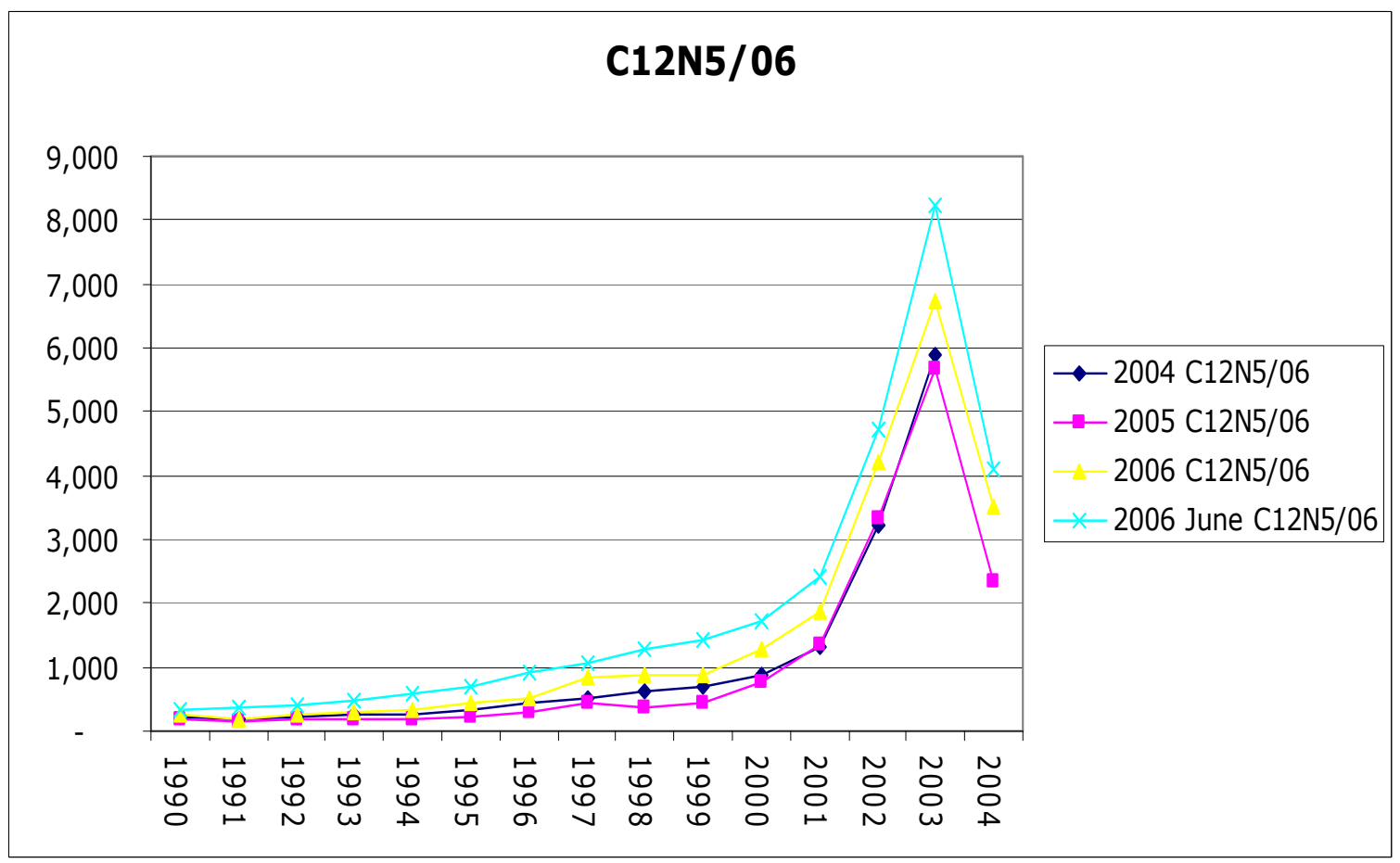


Figure 21

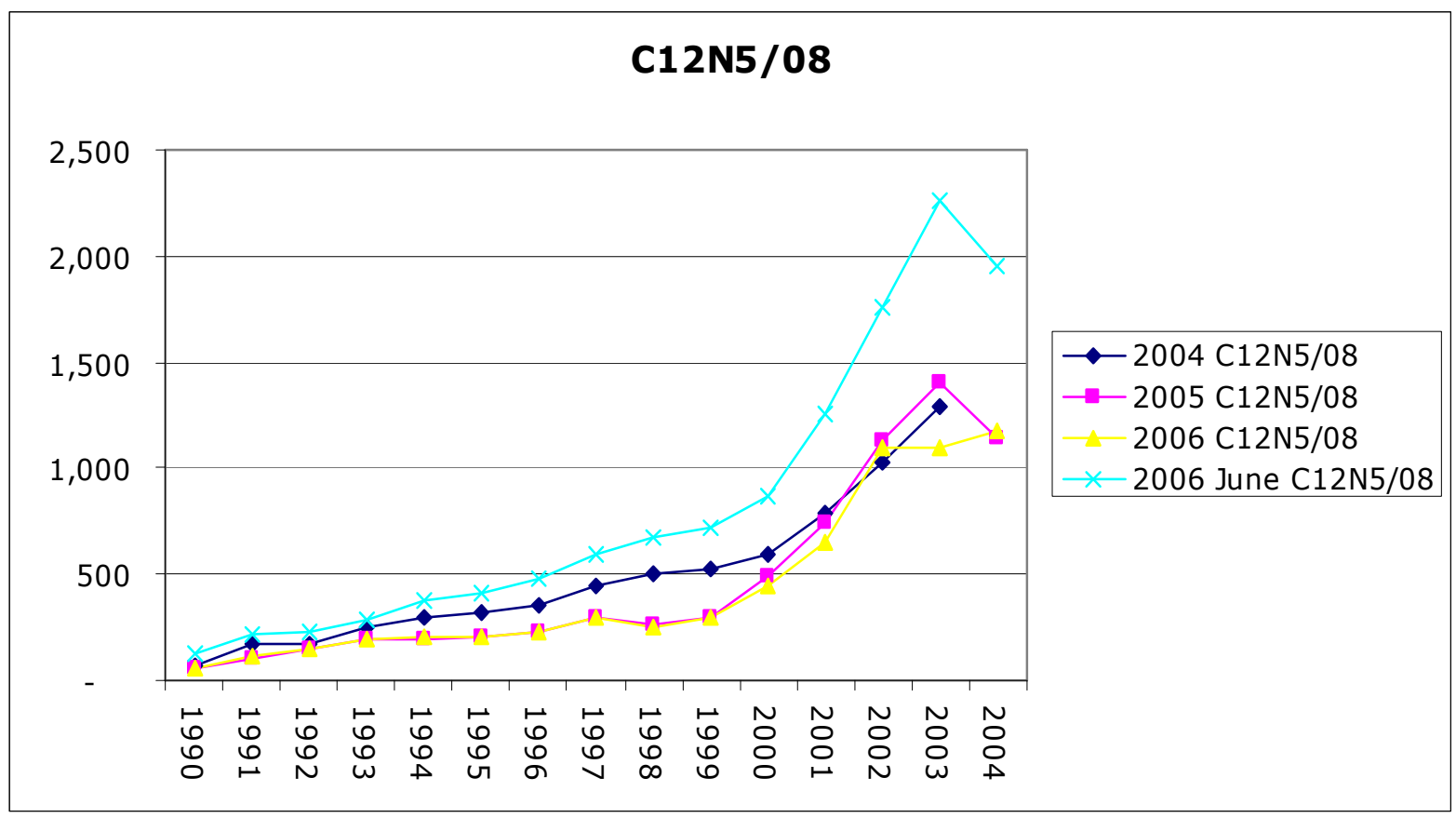

Figure 22

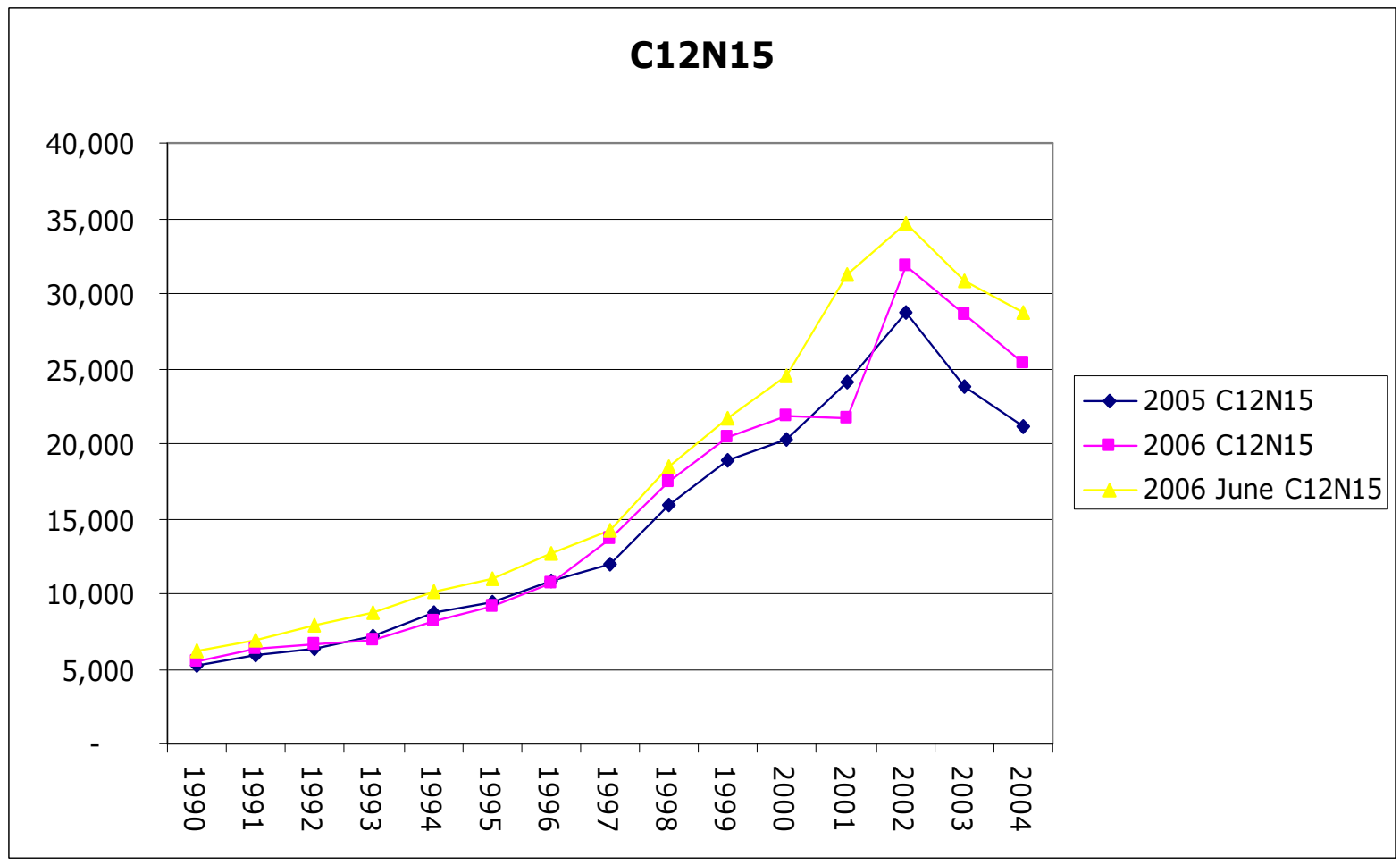


Figure 23

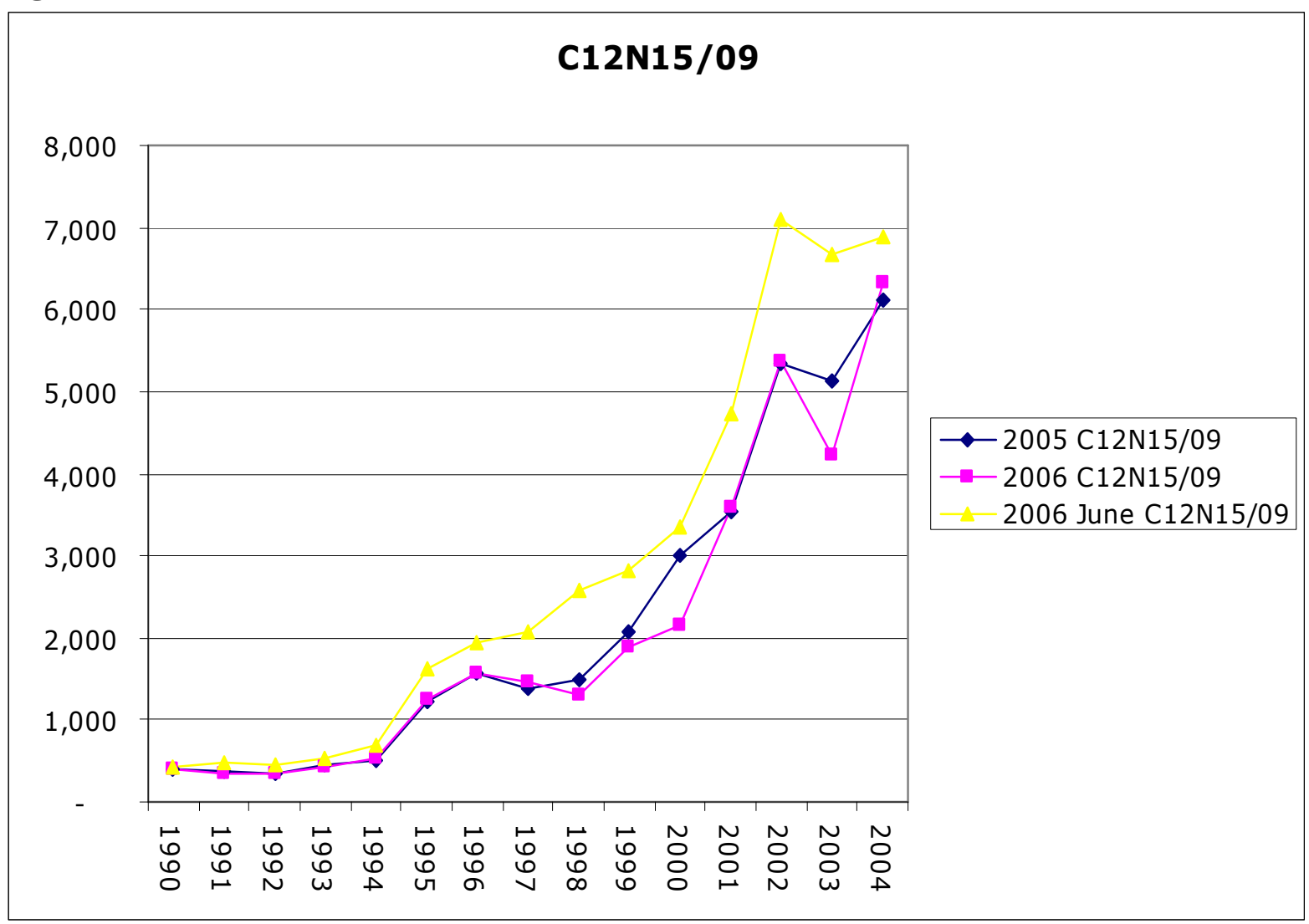

Figure 24

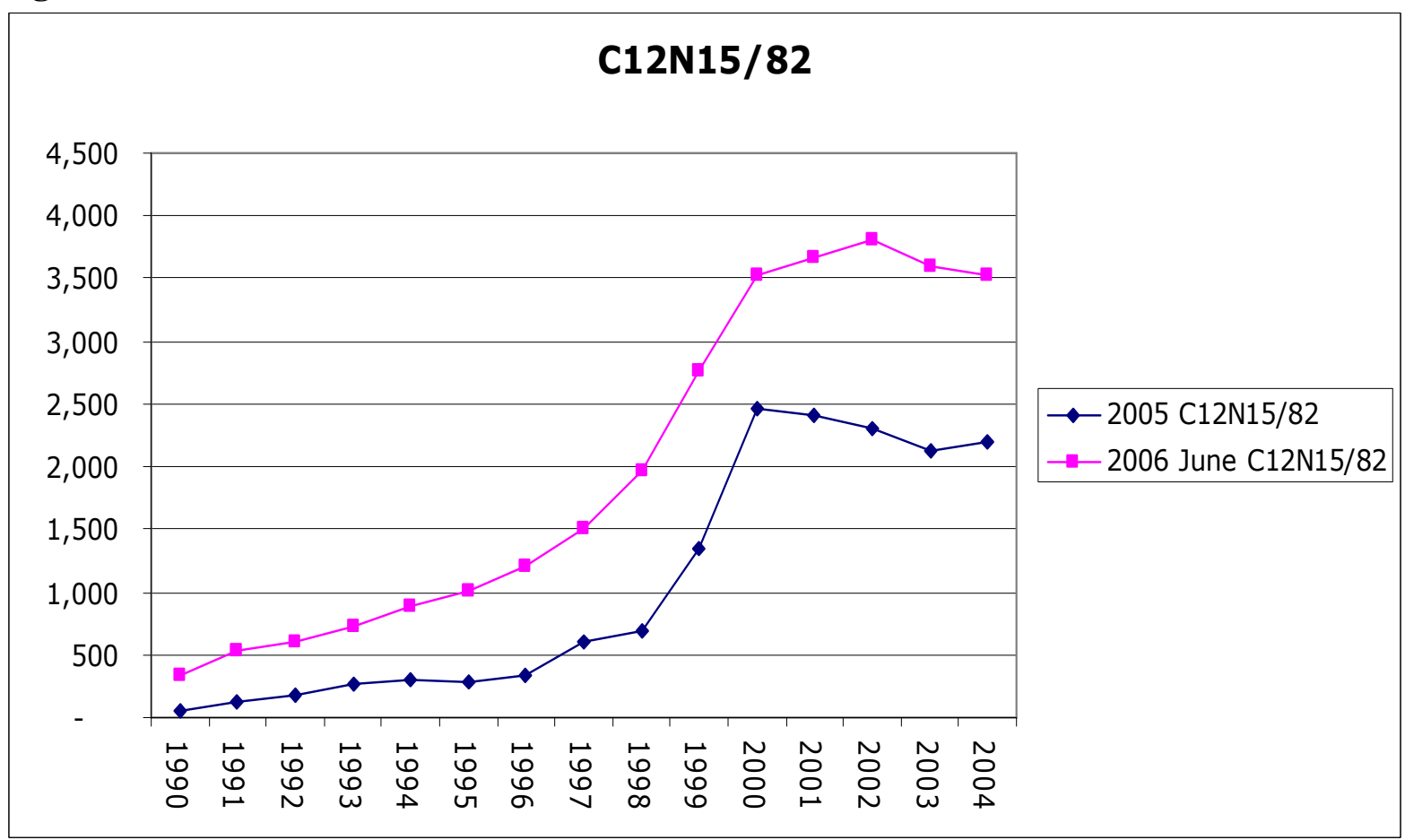


GSP Genomics, Society and Policy

Figure 25

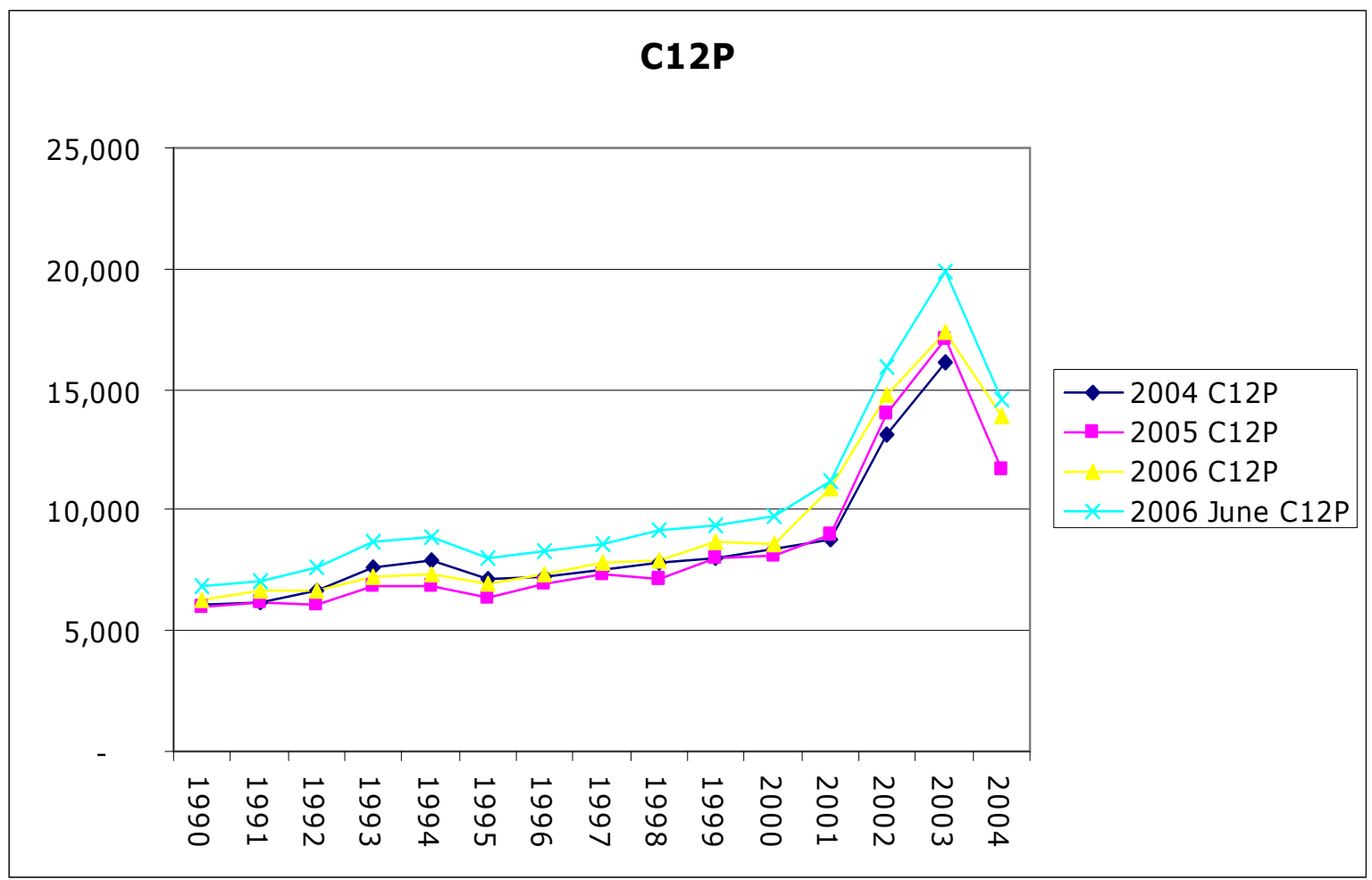

Figure 26

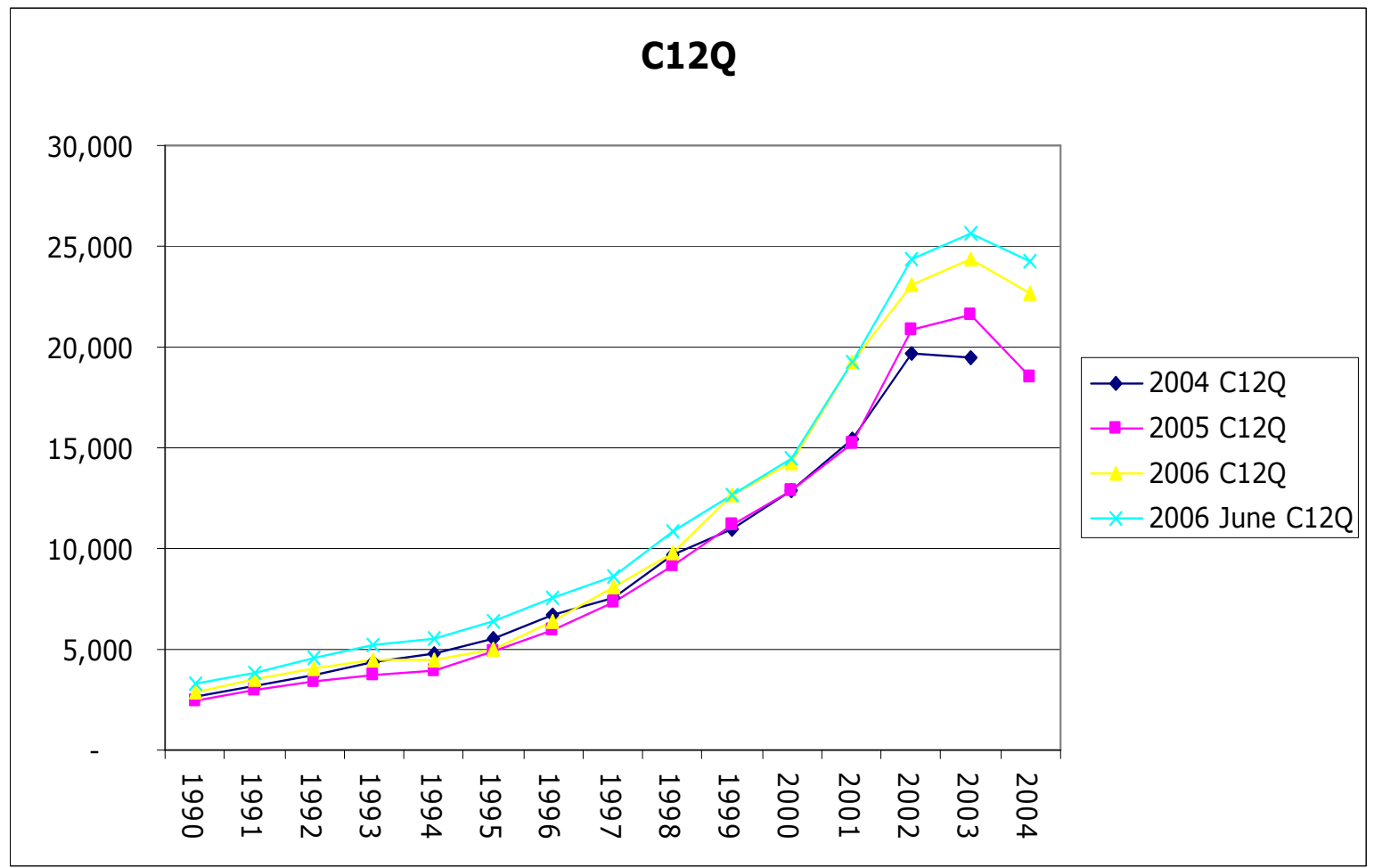


GSP Genomics, Society and Policy

Figure 27

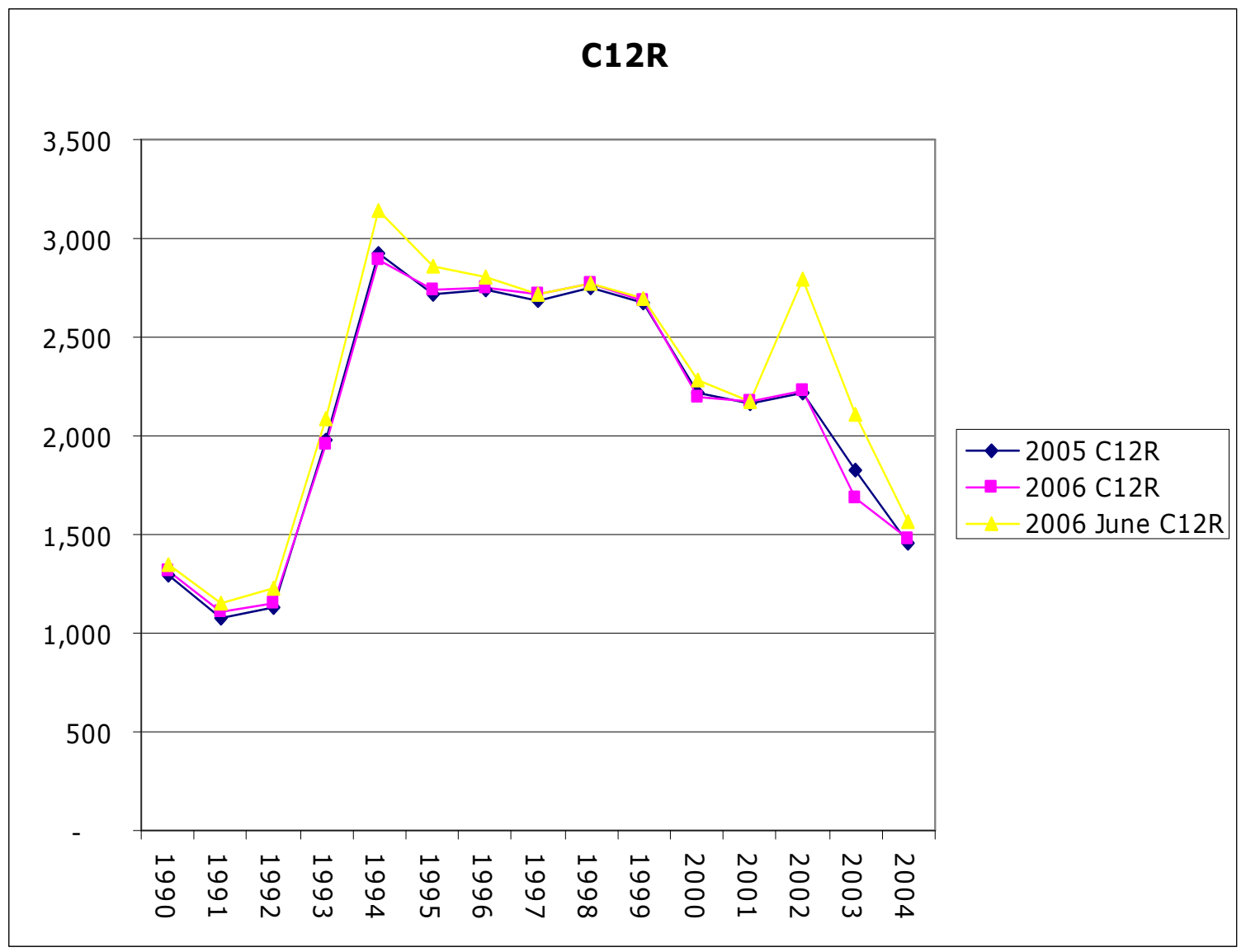

Figure 28

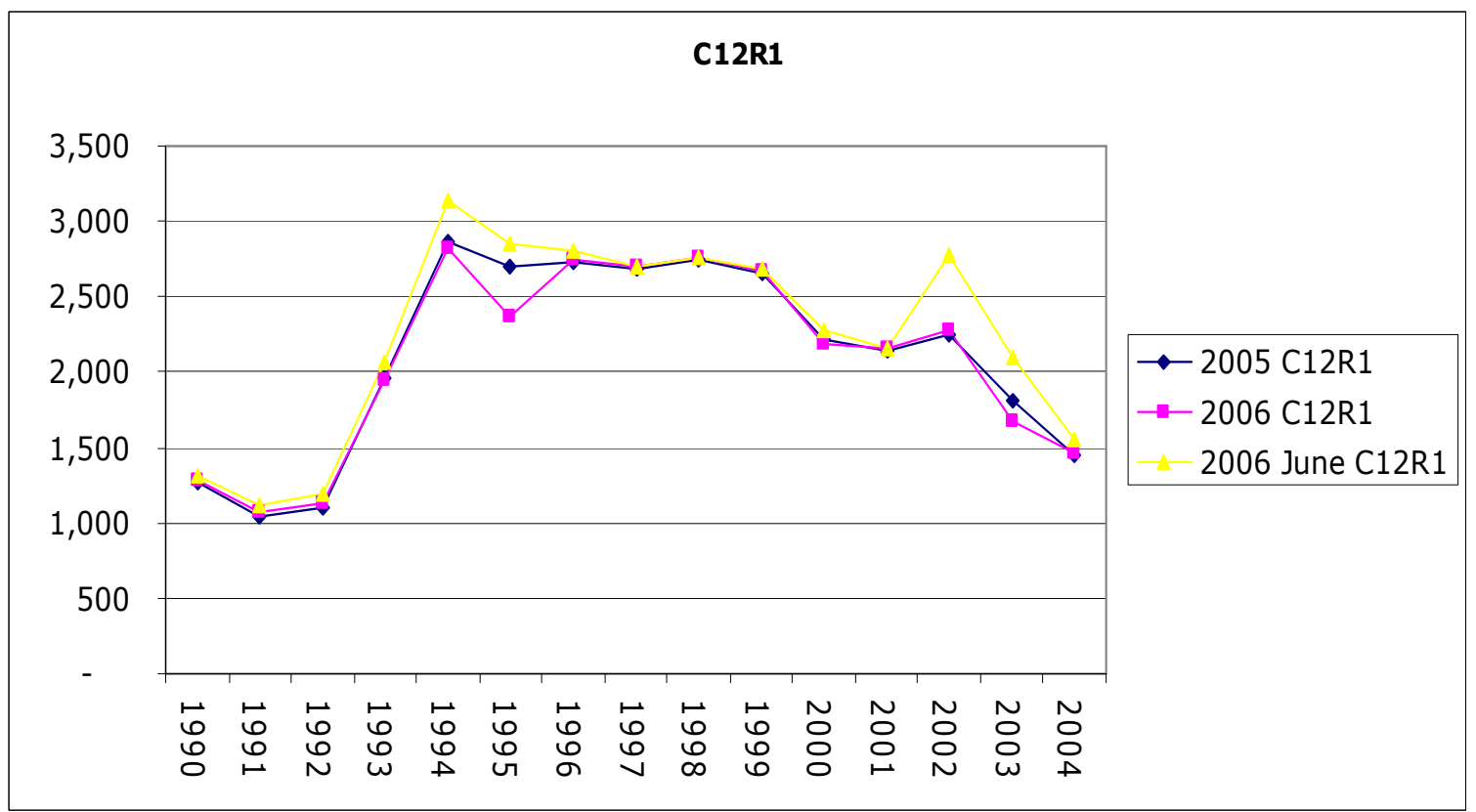


Figure 29

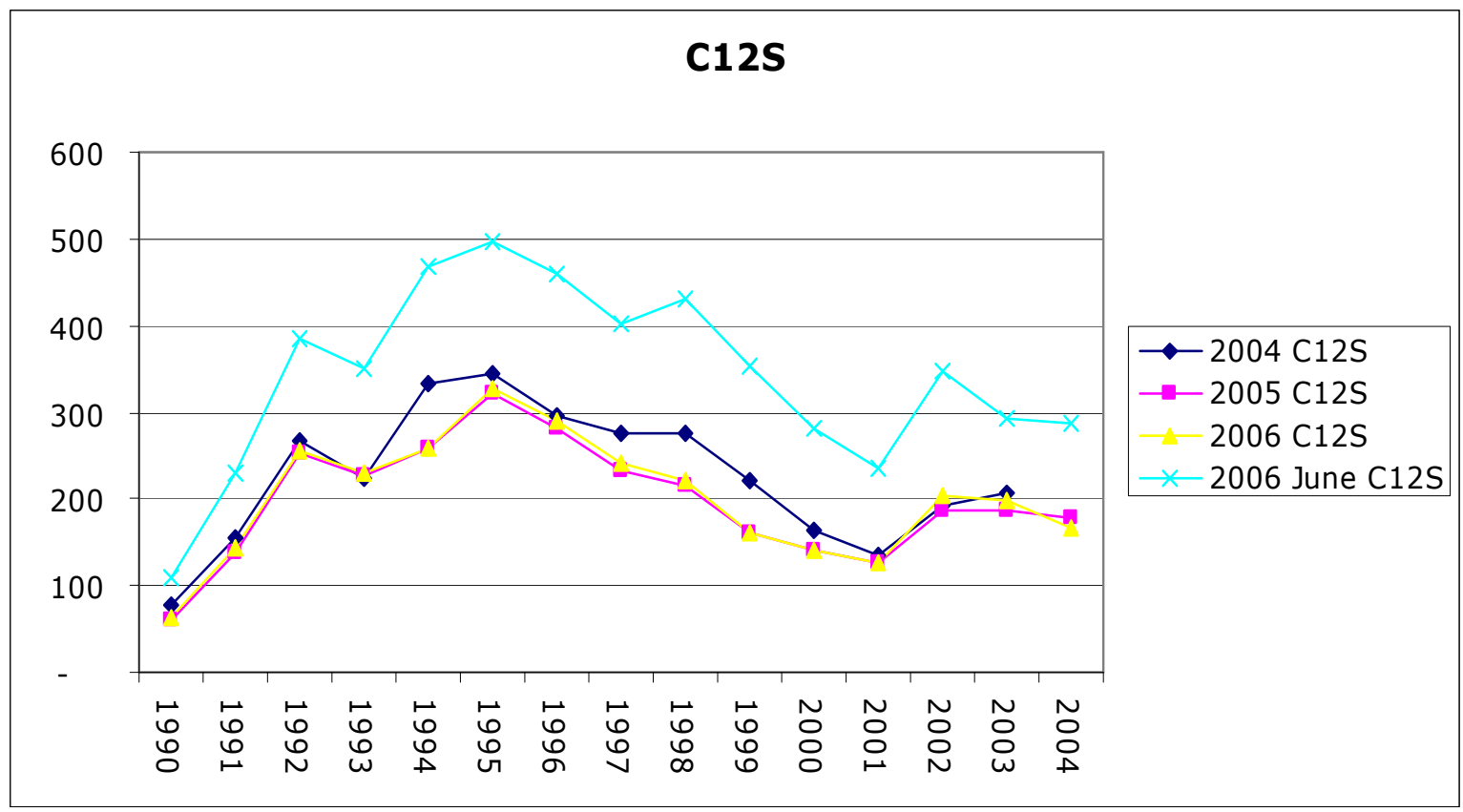

Figure 30

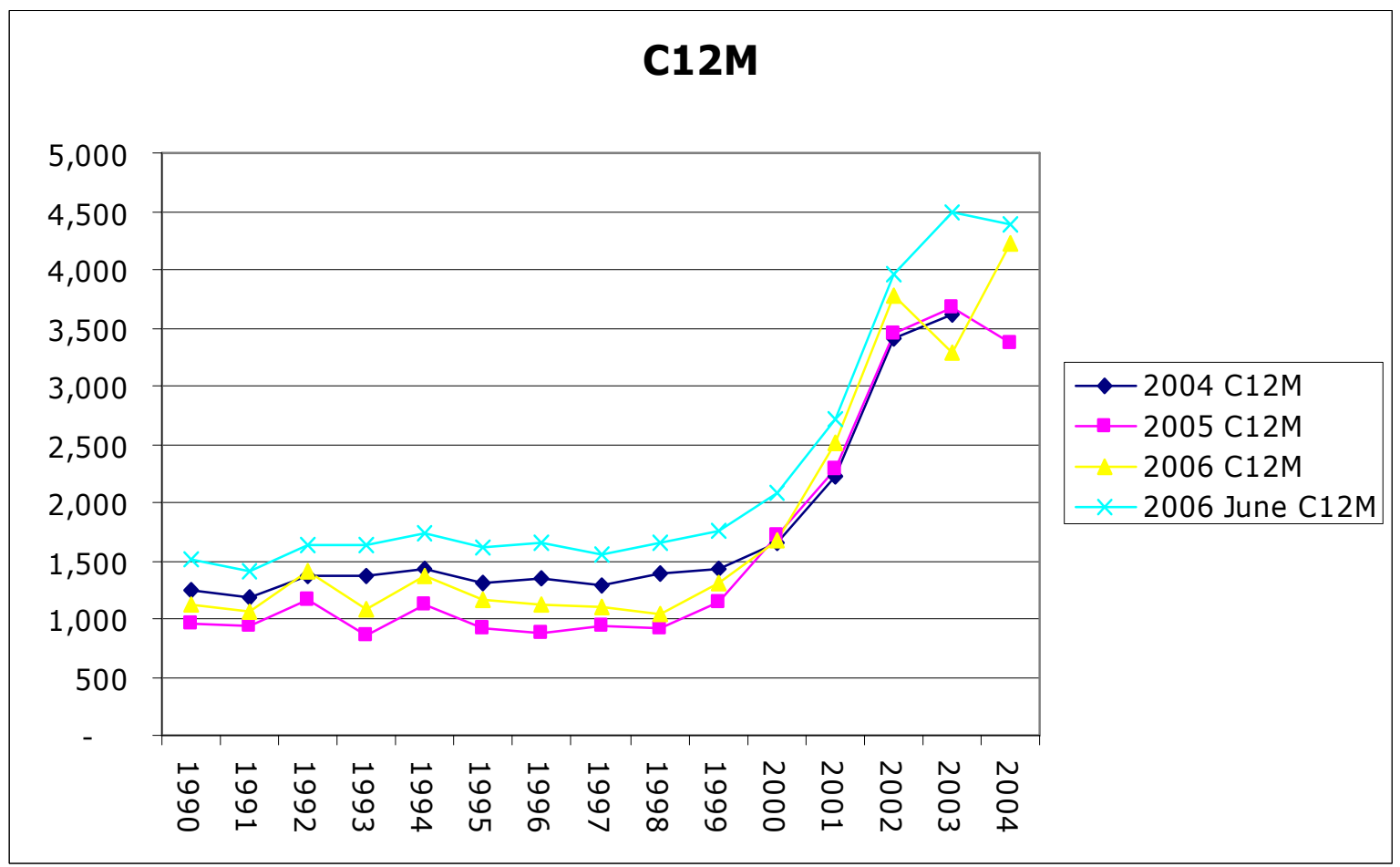


GSP Genomics, Society and Policy online 2006, Vol.2, No.2, pp.92-202

\section{Figure 31}

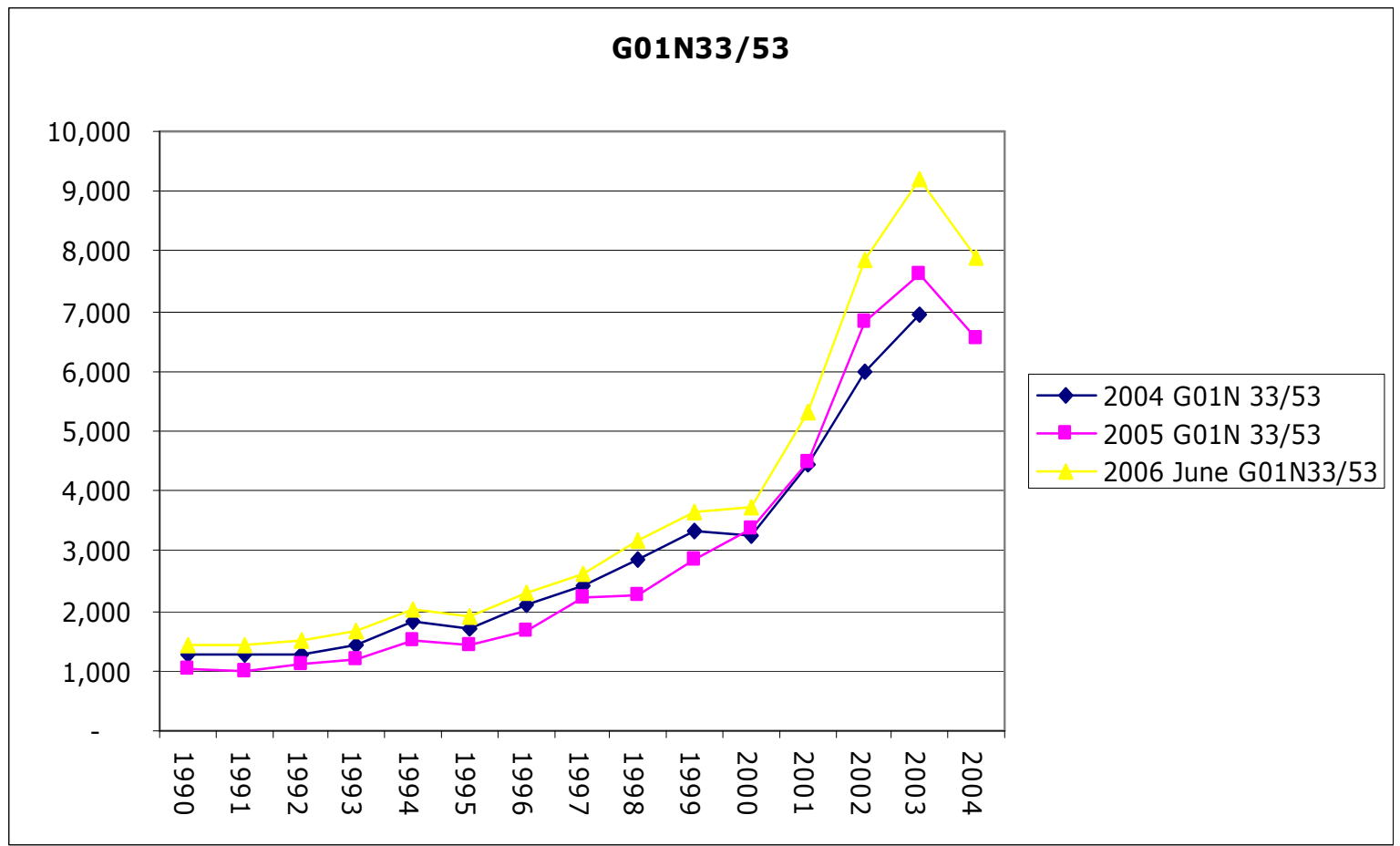


GSP Genomics, Society and Policy online 2006, Vol.2, No.2, pp.92-202

\section{Data coverage tables}

Source: European Patent Office esp@cenet database.

http://ep.espacenet.com/help?locale=en_EP\&method=handleHelpTopic\&topic=detailedcoverage

Database Coverage Summary Calculation by Kind Code (July 2006)

For Kind Codes See WIPO Standard ST. 16.

Note coverage tables exclude XP documents (non-patent literature).

A, B or C

$32,401,382$

$\mathrm{B}$ - second level patent publication

$6,573,043$

C - third level patent publication

$3,210,828$

D Germany - Granted European Patent

766,070

$\mathrm{T}$ (Translations)

$1,429,522$

E (Reissues)

91,489

F (Reexamined)

1,813

$\mathrm{G}$ (Singapore - type assumed patent)

10,238

L (Patent abstract publication)

2,122

P (Plant Patent US)

62,924

M (Special medicine patent FR)

3,607

R -Separately published search report

13,236

$\underline{\text { Sub-Total }}$

$\underline{44,566,274}$

Other

$\mathrm{H}$ - Registrations (US)

664

$S$ - Design patent documents

87,863

U (Utility Model First Level)

$5,051,989$

Y (Utility Model Second Level)

$1,167,989$

Sub-Total

$\underline{6,308,505}$

$\underline{\text { Total }}$

$\underline{50,874,779}$ 
Table VIII. Background Data for Database Summary Calculation

Source: European Patent Office esp@ @enet database.

http://ep.espacenet.com/help?locale=en_EP\&method=handleHelpTopic\&topic=detailedcoverage

The following table gives an overview of the contents of the worldwide database

Explanations of the table fields

Country: country code.

Code: kind code.

Documents: Number of documents of that kind code available in the worldwide database.

Oldest: oldest document of that type having a publication date available. This does not necessarily correspond to the oldest document in the database as usually this one does not contain a populated publication date.

Most recent: the most recent document available when the monthly screening has taken place.

Note: when no kind code is specified, it means A, B or C documents.

Table is current as of 13 July 2006

\begin{tabular}{|c|c|c|c|c|c|c|}
\hline Country & Code & Documents & Oldest & & Most recent & \\
\hline \multirow{33}{*}{ Second level } & $\mathrm{B}$ & 409,950 & AT124B & $(25 / 08 / 1899)$ & AT501082B & $(15 / 06 / 2006)$ \\
\hline & $\mathrm{B}$ & 317,987 & AU113576B & $(30 / 07 / 1941)$ & AU2004240260B & $(22 / 06 / 2006)$ \\
\hline & $\mathrm{B}$ & 102 & BA96066B & $(06 / 03 / 1998)$ & BA98222B & $(02 / 08 / 1999)$ \\
\hline & $\mathrm{B}$ & 4,685 & BG60191 & $(30 / 12 / 1993)$ & BG64720 & $(30 / 12 / 2005)$ \\
\hline & $\mathrm{B}$ & 370 & CA924561 & $(17 / 04 / 1973)$ & CA1340121 & $(10 / 11 / 1998)$ \\
\hline & $\mathrm{B}$ & 2,234 & CH547897 & $(11 / 04 / 1974)$ & CH690157 & $(15 / 11 / 2000)$ \\
\hline & $\mathrm{B}$ & 19,497 & $\mathrm{CN} 85100001 \mathrm{~B}$ & $(10 / 09 / 1985)$ & CN1019872B & $(30 / 12 / 1992)$ \\
\hline & $\mathrm{B}$ & 129,593 & CS110525 & $(15 / 04 / 1964)$ & CS292695 & $(12 / 11 / 2003)$ \\
\hline & $\mathrm{B}$ & 1,104 & CU20602 & $(13 / 02 / 1974)$ & CU22424 & $(11 / 12 / 1995)$ \\
\hline & $\mathrm{B}$ & 18,368 & CZ277680 & $(17 / 03 / 1993)$ & CZ296057 & $(14 / 12 / 2005)$ \\
\hline & $\mathrm{B}$ & 568,160 & DEST2616 & $(19 / 07 / 1951)$ & DE102005024063 & $(06 / 07 / 2006)$ \\
\hline & $\mathrm{B}$ & 65,110 & DK6487B & $(11 / 04 / 1904)$ & DK176107B & $(26 / 06 / 2006)$ \\
\hline & $\mathrm{B}$ & 6,519 & EA7 & $(01 / 07 / 1996)$ & EA6519 & $(29 / 12 / 2005)$ \\
\hline & $\mathrm{B}$ & 1,723 & EE2977 & $(15 / 02 / 1996)$ & EE4654 & $(15 / 06 / 2006)$ \\
\hline & $\mathrm{B}$ & 806,540 & EP0000010 & $(09 / 01 / 1980)$ & EP1625185 & $(05 / 07 / 2006)$ \\
\hline & $\mathrm{B}$ & 22,456 & ES2032239 & $(16 / 08 / 1993)$ & ES2244319 & $(16 / 06 / 2006)$ \\
\hline & $\mathrm{B}$ & 76,073 & FI40001B & $(31 / 05 / 1968)$ & FI117080B & $(31 / 05 / 2006)$ \\
\hline & $\mathrm{B}$ & 565,819 & FR2024873 & $(03 / 11 / 1972)$ & FR2873597 & $(07 / 07 / 2006)$ \\
\hline & $\mathrm{B}$ & 295,522 & GB2000004 & $(06 / 01 / 1982)$ & GB2419376 & $(05 / 07 / 2006)$ \\
\hline & $\mathrm{B}$ & 4,764 & GR1000001 & $(31 / 10 / 1989)$ & GR1005089 & $(28 / 12 / 2005)$ \\
\hline & $\mathrm{B}$ & 2,596 & HR920188 & $(31 / 12 / 1995)$ & HR20040291 & $(30 / 06 / 2006)$ \\
\hline & $\mathrm{B}$ & 62,121 & HU162185 & $(29 / 01 / 1973)$ & HU224764 & $(30 / 01 / 2006)$ \\
\hline & $\mathrm{B}$ & 238 & ID837 & $(29 / 07 / 1992)$ & ID1055 & $(30 / 10 / 1996)$ \\
\hline & $\mathrm{B}$ & 37,829 & IE17383 & $(09 / 01 / 1948)$ & IE83868 & $(20 / 04 / 2005)$ \\
\hline & $\mathrm{B}$ & $1,486,859$ & JP36008730B & $(26 / 06 / 1961)$ & JP10747284B & $(06 / 05 / 1998)$ \\
\hline & $\mathrm{B}$ & 261,391 & KR8000166 & $(10 / 03 / 1980)$ & KR447719 & $(06 / 04 / 2005)$ \\
\hline & $\mathrm{B}$ & 2,303 & LT3001 & $(25 / 08 / 1994)$ & LT5303 & $(27 / 12 / 2005)$ \\
\hline & $\mathrm{B}$ & 3,238 & LV10001 & $(20 / 10 / 1994)$ & LV13372 & $(20 / 12 / 2005)$ \\
\hline & $\mathrm{B}$ & 1,049 & MD1 & $(31 / 01 / 1994)$ & MD3063 & $(31 / 05 / 2006)$ \\
\hline & $\mathrm{B}$ & 11,681 & MX165914 & $(09 / 12 / 1982)$ & MX226742 & $(16 / 03 / 2005)$ \\
\hline & $\mathrm{B}$ & 51,528 & NL24297B & $(15 / 12 / 1924)$ & NL195034B & $(01 / 08 / 2003)$ \\
\hline & $\mathrm{B}$ & 87,127 & NO155347B & $(08 / 12 / 1966)$ & NO321713B & $(26 / 06 / 2006)$ \\
\hline & $\mathrm{B}$ & 111,678 & PL45687B & $(13 / 03 / 1962)$ & PL191342B & $(28 / 04 / 2006)$ \\
\hline
\end{tabular}


GSP Genomics, Society and Policy online 2006, Vol.2, No.2, pp.92-202

\begin{tabular}{|c|c|c|c|c|c|c|}
\hline Country & Code & Documents & Oldest & & Most recent & \\
\hline & B & 26,143 & РT66476 & $(12 / 03 / 1976)$ & PT102929 & (30/09/2005) \\
\hline & B & 18,404 & RO102338 & $(20 / 08 / 1991)$ & RO120682 & $(30 / 05 / 2006)$ \\
\hline & $\mathrm{B}$ & 170,658 & SE205247 & $(07 / 06 / 1966)$ & SE470607 & $(17 / 04 / 2000)$ \\
\hline & $\mathrm{B}$ & 572 & SI8111622 & $(30 / 06 / 1998)$ & SI9720005 & $(28 / 02 / 2006)$ \\
\hline & B & 7,441 & SK277680B & $(08 / 12 / 1993)$ & SK285127B & $(01 / 06 / 2006)$ \\
\hline & $\mathrm{B}$ & 905,282 & US3367320 & $(29 / 12 / 1981)$ & US7073200 & $(04 / 07 / 2006)$ \\
\hline & $\mathrm{B}$ & 8,329 & WO9817166 & $(01 / 10 / 1998)$ & WO2006056260 & $(06 / 07 / 2006)$ \\
\hline & & $6,573,043$ & & & & \\
\hline \multirow[t]{11}{*}{ Third level } & $\mathrm{C}$ & 192,351 & CA1200490 & $(11 / 02 / 1986)$ & CA2486778 & (13/06/2006) \\
\hline & $\mathrm{C}$ & 227,103 & CN1019873C & $(06 / 01 / 1993)$ & CN1247064C & $(22 / 03 / 2006)$ \\
\hline & $\mathrm{C}$ & $1,160,245$ & DE3543 & $(09 / 01 / 1879)$ & DE19903836 & $(06 / 07 / 2006)$ \\
\hline & $\mathrm{C}$ & 146,498 & DK1C & $(25 / 03 / 1895)$ & DK166105C & $(28 / 06 / 2004)$ \\
\hline & $\mathrm{C}$ & 49,600 & FI37062C & $(31 / 08 / 1968)$ & FI98384C & $(07 / 07 / 2005)$ \\
\hline & $\mathrm{C}$ & $1,198,487$ & JP746395C & $(23 / 10 / 1974)$ & JP2119385C & $(06 / 12 / 1996)$ \\
\hline & $\mathrm{C}$ & 59,330 & NL90C & $(02 / 01 / 1914)$ & NL1028882C & $(18 / 04 / 2006)$ \\
\hline & $\mathrm{C}$ & 49,845 & NO173699C & $(19 / 01 / 1909)$ & NO174814C & $(15 / 01 / 2004)$ \\
\hline & $\mathrm{C}$ & 126,609 & SE3819 & (30/09/1892) & SE527871 & $(27 / 06 / 2006)$ \\
\hline & $\mathrm{C}$ & 760 & UA36210 & $(17 / 01 / 2005)$ & UA74120 & $(17 / 10 / 2005)$ \\
\hline & & $3,210,828$ & & & & \\
\hline $\begin{array}{l}\text { Granted, European } \\
\text { Patent number in } \\
\text { bulletin (Germany) }\end{array}$ & $\mathrm{D}$ & 766,070 & DE1710263D & $(18 / 03 / 1971)$ & DE602005000012D & $(06 / 07 / 2006)$ \\
\hline \multirow[t]{3}{*}{$\begin{array}{l}\text { Reissue } \\
\text { patents/certificates } \\
\text { of addition or } \\
\text { amendments } \\
\end{array}$} & $\mathrm{E}$ & 74,108 & FR1983E & $(01 / 01 / 1900)$ & FR96688E & $(03 / 11 / 1978)$ \\
\hline & $\mathrm{E}$ & 4,770 & MX2938E & $(02 / 01 / 1980)$ & MX7734E & $(27 / 06 / 1991)$ \\
\hline & $\mathrm{E}$ & 12,611 & USRE892E & $(07 / 02 / 1860)$ & USRE39157E & $(04 / 07 / 2006)$ \\
\hline \multirow[t]{8}{*}{$\begin{array}{l}\text { Certificates of } \\
\text { reexamined patents }\end{array}$} & $\mathrm{F}$ & 76 & FR6F & $(08 / 01 / 1962)$ & FR357F & $(27 / 07 / 1973)$ \\
\hline & $\mathrm{F}$ & 1,737 & MD62F & (30/09/1994) & MD3064F & (31/05/2006) \\
\hline & $\mathrm{G}$ & 10,238 & SG1082G & $(25 / 02 / 1983)$ & SG37095G & $(01 / 09 / 1995)$ \\
\hline & $\mathrm{H}$ & 2,122 & USH1H & $(03 / 12 / 1985)$ & USH2162H & $(04 / 07 / 2006)$ \\
\hline & $\mathrm{L}$ & 62,924 & IE10651L & $(03 / 01 / 1930)$ & IE980730L & $(10 / 02 / 1999)$ \\
\hline & M & 3,607 & FR664M & $(10 / 07 / 1960)$ & FR8495M & $(27 / 07 / 1973)$ \\
\hline & $\mathrm{P}$ & 13,236 & US3987P & $(14 / 12 / 1976)$ & US16774P & $(04 / 07 / 2006)$ \\
\hline & $\mathrm{R}$ & 664 & LT2001R & $(15 / 10 / 1992)$ & LT2664R & $(25 / 04 / 1994)$ \\
\hline
\end{tabular}


GSP Genomics, Society and Policy online 2006, Vol.2, No.2, pp.92-202

\begin{tabular}{|c|c|c|c|c|c|c|}
\hline Country & Code & Documents & Oldest & & Most recent & \\
\hline & S & 87,863 & USD65880S & $(28 / 10 / 1924)$ & USD524510S & $(04 / 07 / 2006)$ \\
\hline \multirow[t]{12}{*}{ Translations } & $\mathrm{T}$ & 324,684 & AT1T & $(15 / 11 / 1980)$ & AT324774T & $(15 / 05 / 2006)$ \\
\hline & $\mathrm{T}$ & 102 & BE2T & $(07 / 12 / 1979)$ & BE114T & $(15 / 06 / 1993)$ \\
\hline & $\mathrm{T}$ & 1 & CN1084394T & $(08 / 05 / 2002)$ & CN1084394T & $(08 / 05 / 2002)$ \\
\hline & $\mathrm{T}$ & 408,339 & DE2953699T & $(18 / 06 / 1979)$ & DE602004000303T & $(06 / 07 / 2006)$ \\
\hline & $\mathrm{T}$ & 74,346 & DK380312T & $(01 / 08 / 1990)$ & DK1592565T & $(03 / 07 / 2006)$ \\
\hline & $\mathrm{T}$ & 203,352 & ES266162T & $(16 / 01 / 1983)$ & ES2255330T & $(16 / 06 / 2006)$ \\
\hline & $\mathrm{T}$ & 28,147 & GR88300001T & $(18 / 10 / 1988)$ & GR2001300079T & $(31 / 01 / 2002)$ \\
\hline & $\mathrm{T}$ & 346,009 & JP54500001T & $(26 / 07 / 1979)$ & JP2006514819T & $(11 / 05 / 2006)$ \\
\hline & $\mathrm{T}$ & 1 & NL8020180T & $(03 / 08 / 1981)$ & NL8020180T & $(03 / 08 / 1981)$ \\
\hline & $\mathrm{T}$ & 26,231 & PT498721T & $(28 / 04 / 2000)$ & PT1580446T & $(30 / 11 / 2005)$ \\
\hline & $\mathrm{T}$ & 5,778 & SI619406T & $(31 / 10 / 1997)$ & SI1525420T & $(28 / 02 / 2006)$ \\
\hline & $\mathrm{T}$ & 12,532 & TR970231T & $(21 / 03 / 1997)$ & TR200505223T & $(21 / 04 / 2006)$ \\
\hline Total T & & $1,429,522$ & & & & \\
\hline Utility Models & $\mathrm{U}$ & 8,355 & AT1U & $(25 / 07 / 1994)$ & AT8361U & $(15 / 06 / 2006)$ \\
\hline \multirow[t]{18}{*}{$\begin{array}{l}\text { First publication } \\
\text { level }\end{array}$} & $\mathrm{U}$ & 1,116 & BG95859U & $(18 / 01 / 1994)$ & BG109149U & $(30 / 12 / 2005)$ \\
\hline & $\mathrm{U}$ & 75,934 & BR5500123U & $(05 / 08 / 1975)$ & BRMU8502403U & $(13 / 06 / 2006)$ \\
\hline & $\mathrm{U}$ & 124,288 & CN85200999U & $(09 / 04 / 1985)$ & CN2126504U & $(30 / 12 / 1992)$ \\
\hline & $\mathrm{U}$ & 6,709 & CZ6492U & $(04 / 09 / 1997)$ & CZ15984U & $(14 / 12 / 2005)$ \\
\hline & $\mathrm{U}$ & 922,669 & DE1289165U & $(08 / 02 / 1931)$ & DE212004000045U & $(08 / 06 / 2006)$ \\
\hline & $\mathrm{U}$ & 5,043 & DK9200001U & $(28 / 08 / 1992)$ & DK200600139U & $(23 / 06 / 2006)$ \\
\hline & $\mathrm{U}$ & 132,239 & ES245540U & $(01 / 05 / 1959)$ & ES1062489U & $(16 / 06 / 2006)$ \\
\hline & $\mathrm{U}$ & 6,871 & FI1U & $(03 / 02 / 1992)$ & FI7100U & $(31 / 05 / 2006)$ \\
\hline & $\mathrm{U}$ & 1,214 & GR88200217U & $(19 / 01 / 1990)$ & GR2001200024U & $(31 / 01 / 2002)$ \\
\hline & $\mathrm{U}$ & 3,001 & HU1U & $(28 / 08 / 1992)$ & HU3038U & $(30 / 01 / 2006)$ \\
\hline & $\mathrm{U}$ & $3,721,483$ & JP46000001U & $(13 / 09 / 1971)$ & JP2001000044U & $(26 / 12 / 2001)$ \\
\hline & $\mathrm{U}$ & 14,734 & KR7800006U & $(10 / 01 / 1978)$ & KR9814477U & $(05 / 06 / 1998)$ \\
\hline & $\mathrm{U}$ & 2,859 & PH5097U & $(02 / 12 / 1981)$ & PH8499U & $(23 / 12 / 1997)$ \\
\hline & $\mathrm{U}$ & 11,767 & PL100599U & $(08 / 01 / 1996)$ & PL115371U & $(19 / 09 / 2005)$ \\
\hline & $\mathrm{U}$ & 2,785 & PT6095U & $(23 / 06 / 1967)$ & PT10046U & $(30 / 11 / 2005)$ \\
\hline & $\mathrm{U}$ & 6,805 & TR960121U & $(21 / 06 / 1996)$ & TR200601427U & $(21 / 04 / 2006)$ \\
\hline & $\mathrm{U}$ & 52 & VN1U & $(24 / 09 / 1989)$ & VN167U & $(25 / 10 / 1996)$ \\
\hline & $\mathrm{U}$ & 4,065 & UA6429U & $(17 / 01 / 2005)$ & UA10058U & $(17 / 10 / 2005)$ \\
\hline Total U & & $5,051,989$ & & & & \\
\hline Utility models & $\mathrm{Y}$ & 764 & BG51920Y & $(30 / 09 / 1994)$ & BG781Y & $(30 / 12 / 2005)$ \\
\hline \multirow[t]{8}{*}{$\begin{array}{l}\text { Second publication } \\
\text { level }\end{array}$} & $\mathrm{Y}$ & 639,932 & $\mathrm{CN} 2126505 \mathrm{Y}$ & $(06 / 01 / 1993)$ & CN2766491Y & $(22 / 03 / 2006)$ \\
\hline & $\mathrm{Y}$ & 203,455 & ES120641Y & $(01 / 01 / 1968)$ & ES1061638Y & $(16 / 06 / 2006)$ \\
\hline & $\mathrm{Y}$ & 1,658 & GR2000001Y & $(16 / 12 / 1988)$ & GR2002393Y & $(12 / 02 / 2002)$ \\
\hline & $\mathrm{Y}$ & 198,949 & KR8000075Y & $(28 / 01 / 1980)$ & KR246513Y & $(31 / 10 / 2001)$ \\
\hline & $\mathrm{Y}$ & 154 & MX8Y & $(09 / 10 / 1992)$ & MX173Y & $(16 / 05 / 1994)$ \\
\hline & $\mathrm{Y}$ & 8,502 & PL53707Y & $(31 / 01 / 1996)$ & PL62220Y & $(28 / 04 / 2006)$ \\
\hline & $\mathrm{Y}$ & 1,491 & PT6095Y & $(13 / 02 / 1969)$ & РT9951Y & $(31 / 05 / 2005)$ \\
\hline & $\mathrm{Y}$ & 113,084 & TW378487Y & $(01 / 01 / 2000)$ & TW269707Y & $(01 / 07 / 2005)$ \\
\hline Total Y & & $1,167,989$ & & & & \\
\hline
\end{tabular}


GSP Genomics, Society and Policy online 2006, Vol.2, No.2, pp.92-202

\begin{tabular}{|c|c|c|c|c|c|c|}
\hline Country & Code & Documents & Oldest & & Most recent & \\
\hline \multicolumn{7}{|c|}{ By country code } \\
\hline $\mathrm{AP}$ & & 1,282 & AP1 & $(03 / 07 / 1985)$ & AP1473 & $(30 / 09 / 2005)$ \\
\hline $\mathrm{AR}$ & & 48,484 & AR192208 & $(08 / 02 / 1973)$ & AR048850 & $(31 / 05 / 2006)$ \\
\hline AT & & 96,818 & AT288854 & $(12 / 08 / 1963)$ & AT501144 & $(15 / 06 / 2006)$ \\
\hline $\mathrm{AU}$ & & 884,399 & AU440426 & $(24 / 01 / 1928)$ & AU2006202386 & $(22 / 06 / 2006)$ \\
\hline $\mathrm{BA}$ & & 113 & BA94018 & $(28 / 12 / 1998)$ & BA98363 & $(14 / 09 / 2001)$ \\
\hline $\mathrm{BE}$ & & 274,732 & BE37789 & $(15 / 09 / 1875)$ & BE1016269 & $(06 / 06 / 2006)$ \\
\hline $\mathrm{BG}$ & & 43,711 & BG16598 & $(15 / 02 / 1973)$ & BG109181 & $(30 / 12 / 2005)$ \\
\hline $\mathrm{BR}$ & & 313,242 & BR7204242 & $(18 / 07 / 1974)$ & BRPI9912005 & $(13 / 06 / 2006)$ \\
\hline $\mathrm{CA}$ & & $1,179,786$ & CA195677 & $(06 / 01 / 1920)$ & CA2530826 & $(17 / 06 / 2006)$ \\
\hline $\mathrm{CH}$ & & 692,128 & $\mathrm{CH} 70$ & $(09 / 01 / 1888)$ & CH695593 & $(30 / 06 / 2006)$ \\
\hline $\mathrm{CN}$ & & 744,671 & CN85100001 & $(10 / 09 / 1985)$ & CN1751550 & $(22 / 03 / 2006)$ \\
\hline $\mathrm{CS}$ & & 42,842 & CS8100212 & $(26 / 02 / 1982)$ & CS9103618 & $(28 / 12 / 1992)$ \\
\hline $\mathrm{CU}$ & & 1,146 & CU33525 & $(13 / 02 / 1974)$ & CU22307 & $(02 / 12 / 1994)$ \\
\hline $\mathrm{CY}$ & & 2,511 & CY1 & $(06 / 05 / 1921)$ & CY2514 & $(23 / 12 / 2005)$ \\
\hline $\mathrm{CZ}$ & & 48,406 & CZ8300867 & $(13 / 01 / 1993)$ & CZ20050602 & $(14 / 12 / 2005)$ \\
\hline $\mathrm{DE}$ & & $1,741,221$ & DE1491381 & $(25 / 04 / 1964)$ & DE102006011226 & $(06 / 07 / 2006)$ \\
\hline DK & & 98,547 & DK69840 & $(22 / 08 / 1949)$ & DK200401907 & $(09 / 06 / 2006)$ \\
\hline EA & & 223 & EA960001 & $(01 / 07 / 1996)$ & EA980486 & $(29 / 10 / 1998)$ \\
\hline $\mathrm{EE}$ & & 4,643 & EE9400002 & $(15 / 12 / 1995)$ & EE200500030 & $(15 / 06 / 2006)$ \\
\hline EG & & 10,213 & EG9971 & $(31 / 01 / 1976)$ & EG23526 & $(29 / 03 / 2006)$ \\
\hline EP & & $1,676,305$ & EP0000001 & $(20 / 12 / 1978)$ & EP1677588 & $(05 / 07 / 2006)$ \\
\hline ES & & 282,569 & ES280605 & $(16 / 12 / 1947)$ & ES2255466 & $(16 / 06 / 2006)$ \\
\hline FI & & 105,642 & FI18668 & $(09 / 03 / 1933)$ & FI20055463 & $(31 / 05 / 2006)$ \\
\hline FR & & $1,966,638$ & FR318205 & $(09 / 10 / 1902)$ & FR2880512 & $(07 / 07 / 2006)$ \\
\hline GB & & $2,216,512$ & GB185900680 & $(05 / 08 / 1859)$ & GB2421882 & $(05 / 07 / 2006)$ \\
\hline GR & & 35,898 & GR57843 & $(04 / 07 / 1977)$ & GR2005100269 & $(31 / 12 / 2005)$ \\
\hline HK & & 52,380 & HK9376 & $(05 / 03 / 1976)$ & HK1076134 & $(09 / 06 / 2006)$ \\
\hline HR & & 8,493 & HR920170 & $(11 / 08 / 1994)$ & HR20060152 & $(30 / 06 / 2006)$ \\
\hline $\mathrm{HU}$ & & 73,550 & HU2984 & $(02 / 03 / 1970)$ & HU0501031 & $(30 / 01 / 2006)$ \\
\hline ID & & 14,348 & ID20298 & $(26 / 11 / 1988)$ & ID30600 & $(03 / 01 / 2002)$ \\
\hline IE & & 15,038 & IE891581 & $(02 / 01 / 1991)$ & IE20050606 & $(22 / 03 / 2006)$ \\
\hline IL & & 64,355 & IL20106 & $(25 / 01 / 1968)$ & IL168574 & $(18 / 12 / 2005)$ \\
\hline IN & & 54,283 & IN84678 & $(02 / 08 / 1975)$ & IN193030 & $(19 / 06 / 2004)$ \\
\hline IT & & 77,452 & IT354856 & $(11 / 12 / 1937)$ & ITRM20030511 & $(02 / 02 / 2004)$ \\
\hline JP & & $9,153,235$ & JP46026238 & $(29 / 07 / 1971)$ & JP2006121880 & $(11 / 05 / 2006)$ \\
\hline $\mathrm{KE}$ & & 1,336 & KE2536 & $(11 / 07 / 1975)$ & KE3892 & $(01 / 09 / 1989)$ \\
\hline KR & & 281,986 & KR7800099 & $(08 / 01 / 1978)$ & KR2005028360 & $(23 / 03 / 2005)$ \\
\hline LT & & 2,500 & LT99 & $(25 / 03 / 1994)$ & LT2005063 & $(27 / 12 / 2005)$ \\
\hline $\mathrm{LU}$ & & 49,784 & LU27693 & $(02 / 06 / 1945)$ & LU91206 & $(30 / 05 / 2006)$ \\
\hline LV & & 3,655 & LV5001 & $(10 / 06 / 1993)$ & LV12838 & $(20 / 05 / 2002)$ \\
\hline $\mathrm{MC}$ & & 2,570 & $\mathrm{MC1}$ & $(13 / 12 / 1957)$ & MC200083 & $(07 / 12 / 2005)$ \\
\hline MD & & 952 & MD930016 & $(30 / 09 / 1994)$ & MD20040196 & $(31 / 05 / 2006)$ \\
\hline MN & & 233 & MN57 & $(20 / 11 / 1972)$ & MN465 & $(15 / 06 / 1989)$ \\
\hline MT & & 545 & MT542 & $(25 / 06 / 1968)$ & MT1078 & $(08 / 05 / 1992)$ \\
\hline MW & & 732 & MW3671 & $(09 / 05 / 1973)$ & MW13093 & $(12 / 10 / 1994)$ \\
\hline MX & & 83,343 & MX143111 & $(14 / 10 / 1980)$ & MXPA99008569 & $(10 / 04 / 2006)$ \\
\hline MY & & 9,618 & MY153 & $(31 / 12 / 1953)$ & MY109280 & $(31 / 12 / 1996)$ \\
\hline NL & & 316,887 & NL190149 & $(17 / 02 / 1924)$ & NL1030316 & $(18 / 04 / 2006)$ \\
\hline $\mathrm{NO}$ & & 148,421 & NO71432 & $(23 / 12 / 1946)$ & NO20055263 & $(12 / 05 / 2006)$ \\
\hline
\end{tabular}

Genomics, Society and Policy, Vol.2 No.2 (2006) ISSN: 1746-5354

(C) ESRC Genomics Network. 
GSP Genomics, Society and Policy

\begin{tabular}{|c|c|c|c|c|c|c|}
\hline Country & Code & Documents & Oldest & & Most recent & \\
\hline $\mathrm{NZ}$ & & 85,556 & NZ171180 & $(06 / 03 / 1978)$ & NZ546316 & $(30 / 06 / 2006)$ \\
\hline $\mathrm{OA}$ & & 11,760 & OA1 & $(15 / 01 / 1966)$ & OA11847 & $(23 / 08 / 2005)$ \\
\hline $\mathrm{PH}$ & & 19,956 & PH9199 & $(03 / 07 / 1975)$ & PH31699 & $(18 / 01 / 1999)$ \\
\hline PL & & 169,703 & PL68374 & $(28 / 02 / 1973)$ & PL373762 & $(05 / 09 / 2005)$ \\
\hline PT & & 38,140 & PT56642 & $(01 / 11 / 1971)$ & PT103313 & $(30 / 11 / 2005)$ \\
\hline RO & & 38,589 & $\mathrm{RO1}$ & $(08 / 01 / 1907)$ & RO102718 & $(22 / 03 / 1993)$ \\
\hline RUC & & 272,924 & RU2000001 & $(15 / 02 / 1993)$ & RU2274965 & $(20 / 04 / 2006)$ \\
\hline SE & & 152,321 & SE122183 & $(13 / 07 / 1948)$ & SE0501863 & $(27 / 06 / 2006)$ \\
\hline SG & & 27,341 & SG39090 & $(17 / 08 / 1990)$ & SG118232 & $(27 / 06 / 2006)$ \\
\hline SI & & 5,731 & SI9100001 & $(27 / 11 / 1992)$ & SI21869 & $(28 / 02 / 2006)$ \\
\hline SK & & 20,875 & SK3893 & $(07 / 07 / 1993)$ & SK51112005 & $(01 / 06 / 2006)$ \\
\hline SU & & $1,162,205$ & SU18207 & $(30 / 09 / 1930)$ & SU1829367 & $(20 / 04 / 2006)$ \\
\hline $\mathrm{TJ}$ & & 164 & TJ96000415 & $(14 / 11 / 1998)$ & TJ20040823 & $(06 / 01 / 2006)$ \\
\hline TR & & 17,145 & TR16570 & $(01 / 01 / 1973)$ & TR200503829 & $(21 / 04 / 2006)$ \\
\hline TWB & & 115,969 & TW211035B & $(11 / 08 / 1993)$ & TW235029B & $(21 / 06 / 2005)$ \\
\hline US & & $5,986,161$ & US11713 & $(19 / 09 / 1854)$ & US2006143744 & $(29 / 06 / 2006)$ \\
\hline $\mathrm{VN}$ & & 112 & VN1 & $(06 / 07 / 1984)$ & VN344 & $(25 / 04 / 1997)$ \\
\hline WO & & $1,140,656$ & WO7800001 & $(19 / 10 / 1978)$ & WO2006072117 & $(06 / 07 / 2006)$ \\
\hline $\mathrm{ZA}$ & & 206,516 & ZA6901534 & $(27 / 01 / 1971)$ & ZA200501578 & $(09 / 12 / 2005)$ \\
\hline DZ & & 16 & DZ2403 & $(04 / 01 / 2005)$ & DZ2783 & $(08 / 10 / 2005)$ \\
\hline GC & & 77 & GC0000093 & $(29 / 06 / 2005)$ & GC0000170 & $(29 / 06 / 2005)$ \\
\hline MA & & 797 & MA27120 & $(03 / 01 / 2005)$ & MA27916 & $(02 / 05 / 2006)$ \\
\hline UA & & 290 & UA72070 & $(17 / 01 / 2005)$ & UA74127 & $(17 / 10 / 2005)$ \\
\hline $\mathrm{A}, \mathrm{B}$ or $\mathrm{C}$ & & $32,401,382$ & & & & \\
\hline
\end{tabular}


Table IX. Worldwide Database - Detailed Coverage (2006 data)

Source: European Patent Office esp@ cenet database

http://ep.espacenet.com/help?locale=en_EP\&method=handleHelpTopic\&topic=detailedcoverage.

The following table gives an overview of the contents of the worldwide database.

Note: XP documents are excluded from the 2006 data and encompassed 4.9 million documents in 2005

Explanations of the table fields

Country: country code.

Code: kind code.

Documents: Number of documents of that kind code available in the worldwide database.

Oldest: oldest document of that type having a publication date available.

This does not necessarily correspond to the oldest document in the database as usually this one does not contain a populated publication date.

Source: European Patent Office esp@ cenet database

Most recent: the most recent document available when the monthly screening has taken place.

Note: when no kind code is specified, it means A, B or C documents

Table is current as of 13 July 2006

\begin{tabular}{|c|c|c|c|c|c|c|}
\hline Country & Code & Documents & Oldest & & Most recent & \\
\hline $\mathrm{AP}$ & & 1,282 & AP1 & $(03 / 07 / 1985)$ & AP1473 & $(30 / 09 / 2005)$ \\
\hline $\mathrm{AR}$ & & 48,484 & AR192208 & $(08 / 02 / 1973)$ & AR048850 & $(31 / 05 / 2006)$ \\
\hline \multirow[t]{4}{*}{ AT } & & 96,818 & AT288854 & $(12 / 08 / 1963)$ & AT501144 & $(15 / 06 / 2006)$ \\
\hline & $\mathrm{B}$ & 409,950 & AT124B & $(25 / 08 / 1899)$ & AT501082B & $(15 / 06 / 2006)$ \\
\hline & $\mathrm{T}$ & 324,684 & AT1T & $(15 / 11 / 1980)$ & AT324774T & $(15 / 05 / 2006)$ \\
\hline & $\mathrm{U}$ & 8,355 & AT1U & $(25 / 07 / 1994)$ & AT8361U & $(15 / 06 / 2006)$ \\
\hline \multirow[t]{2}{*}{$\mathrm{AU}$} & & 884,399 & AU440426 & $(24 / 01 / 1928)$ & AU2006202386 & $(22 / 06 / 2006)$ \\
\hline & $\mathrm{B}$ & 317,987 & AU113576B & $(30 / 07 / 1941)$ & AU2004240260B & $(22 / 06 / 2006)$ \\
\hline \multirow[t]{2}{*}{ BA } & & 113 & BA94018 & $(28 / 12 / 1998)$ & BA98363 & $(14 / 09 / 2001)$ \\
\hline & $\mathrm{B}$ & 102 & BA96066B & $(06 / 03 / 1998)$ & BA98222B & $(02 / 08 / 1999)$ \\
\hline \multirow[t]{2}{*}{$\mathrm{BE}$} & & 274,732 & BE37789 & $(15 / 09 / 1875)$ & BE1016269 & $(06 / 06 / 2006)$ \\
\hline & $\mathrm{T}$ & 102 & BE2T & $(07 / 12 / 1979)$ & BE114T & $(15 / 06 / 1993)$ \\
\hline \multirow[t]{4}{*}{ BG } & & 43,711 & BG16598 & $(15 / 02 / 1973)$ & BG109181 & $(30 / 12 / 2005)$ \\
\hline & $\mathrm{B}$ & 4,685 & BG60191 & $(30 / 12 / 1993)$ & BG64720 & $(30 / 12 / 2005)$ \\
\hline & $\mathrm{U}$ & 1,116 & BG95859U & $(18 / 01 / 1994)$ & BG109149U & $(30 / 12 / 2005)$ \\
\hline & $\mathrm{Y}$ & 764 & BG51920Y & $(30 / 09 / 1994)$ & BG781Y & $(30 / 12 / 2005)$ \\
\hline \multirow[t]{2}{*}{ BR } & & 313,242 & BR7204242 & $(18 / 07 / 1974)$ & BRPI9912005 & $(13 / 06 / 2006)$ \\
\hline & $\mathrm{U}$ & 75,934 & BR5500123U & $(05 / 08 / 1975)$ & BRMU8502403U & $(13 / 06 / 2006)$ \\
\hline \multirow[t]{3}{*}{$\mathrm{CA}$} & & $1,179,786$ & CA195677 & $(06 / 01 / 1920)$ & CA2530826 & $(17 / 06 / 2006)$ \\
\hline & $\mathrm{B}$ & 370 & CA924561 & $(17 / 04 / 1973)$ & CA1340121 & $(10 / 11 / 1998)$ \\
\hline & $\mathrm{C}$ & 192,351 & CA1200490 & $(11 / 02 / 1986)$ & CA2486778 & $(13 / 06 / 2006)$ \\
\hline \multirow[t]{2}{*}{$\mathrm{CH}$} & & 692,128 & $\mathrm{CH} 70$ & $(09 / 01 / 1888)$ & CH695593 & $(30 / 06 / 2006)$ \\
\hline & $\mathrm{B}$ & 2,234 & $\mathrm{CH} 547897$ & $(11 / 04 / 1974)$ & CH690157 & $(15 / 11 / 2000)$ \\
\hline \multirow[t]{6}{*}{$\mathrm{CN}$} & & 744,671 & CN85100001 & $(10 / 09 / 1985)$ & CN1751550 & $(22 / 03 / 2006)$ \\
\hline & $\mathrm{B}$ & 19,497 & CN85100001B & $(10 / 09 / 1985)$ & CN1019872B & $(30 / 12 / 1992)$ \\
\hline & $\mathrm{C}$ & 227,103 & CN1019873C & $(06 / 01 / 1993)$ & CN1247064C & $(22 / 03 / 2006)$ \\
\hline & $\mathrm{T}$ & 1 & CN1084394T & $(08 / 05 / 2002)$ & CN1084394T & $(08 / 05 / 2002)$ \\
\hline & $\mathrm{U}$ & 124,288 & CN85200999U & $(09 / 04 / 1985)$ & $\mathrm{CN} 2126504 \mathrm{U}$ & $(30 / 12 / 1992)$ \\
\hline & $\mathrm{Y}$ & 639,932 & CN2126505Y & $(06 / 01 / 1993)$ & CN2766491Y & $(22 / 03 / 2006)$ \\
\hline $\mathrm{CS}$ & & 42,842 & CS8100212 & $(26 / 02 / 1982)$ & CS9103618 & $(28 / 12 / 1992)$ \\
\hline
\end{tabular}


GSP Genomics, Society and Policy 2006, Vol.2, No.2, pp.92-202

\begin{tabular}{|c|c|c|c|c|c|c|}
\hline Country & Code & Documents & Oldest & & Most recent & \\
\hline & $\mathrm{B}$ & 129,593 & CS110525 & $(15 / 04 / 1964)$ & CS292695 & $(12 / 11 / 2003)$ \\
\hline \multirow[t]{2}{*}{$\mathrm{CU}$} & & 1,146 & CU33525 & $(13 / 02 / 1974)$ & CU22307 & $(02 / 12 / 1994)$ \\
\hline & $\mathrm{B}$ & 1,104 & CU20602 & $(13 / 02 / 1974)$ & CU22424 & $(11 / 12 / 1995)$ \\
\hline $\mathrm{CY}$ & & 2,511 & CY1 & $(06 / 05 / 1921)$ & CY2514 & $(23 / 12 / 2005)$ \\
\hline \multirow[t]{3}{*}{$\mathrm{CZ}$} & & 48,406 & CZ8300867 & (13/01/1993) & CZ20050602 & $(14 / 12 / 2005)$ \\
\hline & $\mathrm{B}$ & 18,368 & CZ277680 & $(17 / 03 / 1993)$ & CZ296057 & $(14 / 12 / 2005)$ \\
\hline & $\mathrm{U}$ & 6,709 & CZ6492U & $(04 / 09 / 1997)$ & CZ15984U & $(14 / 12 / 2005)$ \\
\hline \multirow[t]{6}{*}{$\mathrm{DE}$} & & $1,741,221$ & DE1491381 & $(25 / 04 / 1964)$ & DE102006011226 & $(06 / 07 / 2006)$ \\
\hline & $\mathrm{B}$ & 568,160 & DEST2616 & $(19 / 07 / 1951)$ & DE102005024063 & $(06 / 07 / 2006)$ \\
\hline & $\mathrm{C}$ & $1,160,245$ & DE3543 & $(09 / 01 / 1879)$ & DE19903836 & $(06 / 07 / 2006)$ \\
\hline & $\mathrm{D}$ & 766,070 & DE1710263D & $(18 / 03 / 1971)$ & DE602005000012D & $(06 / 07 / 2006)$ \\
\hline & $\mathrm{T}$ & 408,339 & DE2953699T & $(18 / 06 / 1979)$ & DE602004000303T & $(06 / 07 / 2006)$ \\
\hline & $\mathrm{U}$ & 922,669 & DE1289165U & $(08 / 02 / 1931)$ & DE212004000045U & $(08 / 06 / 2006)$ \\
\hline \multirow[t]{5}{*}{ DK } & & 98,547 & DK69840 & $(22 / 08 / 1949)$ & DK200401907 & $(09 / 06 / 2006)$ \\
\hline & $\mathrm{B}$ & 65,110 & DK6487B & $(11 / 04 / 1904)$ & DK176107B & $(26 / 06 / 2006)$ \\
\hline & $\mathrm{C}$ & 146,498 & DK1C & $(25 / 03 / 1895)$ & DK166105C & $(28 / 06 / 2004)$ \\
\hline & $\mathrm{T}$ & 74,346 & DK380312T & $(01 / 08 / 1990)$ & DK1592565T & $(03 / 07 / 2006)$ \\
\hline & $\mathrm{U}$ & 5,043 & DK9200001U & $(28 / 08 / 1992)$ & DK200600139U & $(23 / 06 / 2006)$ \\
\hline \multirow[t]{2}{*}{ EA } & & 223 & EA960001 & $(01 / 07 / 1996)$ & EA980486 & $(29 / 10 / 1998)$ \\
\hline & $\mathrm{B}$ & 6,519 & EA7 & $(01 / 07 / 1996)$ & EA6519 & $(29 / 12 / 2005)$ \\
\hline \multirow[t]{2}{*}{$\mathrm{EE}$} & & 4,643 & EE9400002 & $(15 / 12 / 1995)$ & EE200500030 & $(15 / 06 / 2006)$ \\
\hline & $\mathrm{B}$ & 1,723 & EE2977 & $(15 / 02 / 1996)$ & EE4654 & $(15 / 06 / 2006)$ \\
\hline EG & & 10,213 & EG9971 & $(31 / 01 / 1976)$ & EG23526 & $(29 / 03 / 2006)$ \\
\hline \multirow[t]{2}{*}{ EP } & & $1,676,305$ & EP0000001 & $(20 / 12 / 1978)$ & EP1677588 & $(05 / 07 / 2006)$ \\
\hline & B & 806,540 & EP0000010 & $(09 / 01 / 1980)$ & EP1625185 & $(05 / 07 / 2006)$ \\
\hline \multirow[t]{5}{*}{ ES } & & 282,569 & ES280605 & $(16 / 12 / 1947)$ & ES2255466 & $(16 / 06 / 2006)$ \\
\hline & $\mathrm{B}$ & 22,456 & ES2032239 & $(16 / 08 / 1993)$ & ES2244319 & $(16 / 06 / 2006)$ \\
\hline & $\mathrm{T}$ & 203,352 & ES266162T & $(16 / 01 / 1983)$ & ES2255330T & $(16 / 06 / 2006)$ \\
\hline & $\mathrm{U}$ & 132,239 & ES245540U & $(01 / 05 / 1959)$ & ES1062489U & $(16 / 06 / 2006)$ \\
\hline & $\mathrm{Y}$ & 203,455 & ES120641Y & $(01 / 01 / 1968)$ & ES1061638Y & $(16 / 06 / 2006)$ \\
\hline \multirow[t]{4}{*}{ FI } & & 105,642 & FI18668 & $(09 / 03 / 1933)$ & FI20055463 & $(31 / 05 / 2006)$ \\
\hline & $\mathrm{B}$ & 76,073 & FI40001B & $(31 / 05 / 1968)$ & FI117080B & $(31 / 05 / 2006)$ \\
\hline & $\mathrm{C}$ & 49,600 & FI37062C & $(31 / 08 / 1968)$ & FI98384C & $(07 / 07 / 2005)$ \\
\hline & $\mathrm{U}$ & 6,871 & FI1U & $(03 / 02 / 1992)$ & FI7100U & $(31 / 05 / 2006)$ \\
\hline \multirow[t]{5}{*}{ FR } & & $1,966,638$ & FR318205 & $(09 / 10 / 1902)$ & FR2880512 & $(07 / 07 / 2006)$ \\
\hline & $\mathrm{B}$ & 565,819 & FR2024873 & $(03 / 11 / 1972)$ & FR2873597 & $(07 / 07 / 2006)$ \\
\hline & E & 74,108 & FR1983E & $(01 / 01 / 1900)$ & FR96688E & $(03 / 11 / 1978)$ \\
\hline & $\mathrm{F}$ & 76 & FR6F & $(08 / 01 / 1962)$ & FR357F & $(27 / 07 / 1973)$ \\
\hline & M & 3,607 & FR664M & $(10 / 07 / 1960)$ & FR8495M & $(27 / 07 / 1973)$ \\
\hline \multirow[t]{2}{*}{ GB } & & $2,216,512$ & GB185900680 & $(05 / 08 / 1859)$ & GB2421882 & $(05 / 07 / 2006)$ \\
\hline & $\mathrm{B}$ & 295,522 & GB2000004 & $(06 / 01 / 1982)$ & GB2419376 & $(05 / 07 / 2006)$ \\
\hline \multirow[t]{5}{*}{ GR } & & 35,898 & GR57843 & $(04 / 07 / 1977)$ & GR2005100269 & $(31 / 12 / 2005)$ \\
\hline & $\mathrm{B}$ & 4,764 & GR1000001 & $(31 / 10 / 1989)$ & GR1005089 & $(28 / 12 / 2005)$ \\
\hline & $\mathrm{T}$ & 28,147 & GR88300001T & (18/10/1988) & GR2001300079T & $(31 / 01 / 2002)$ \\
\hline & $\mathrm{U}$ & 1,214 & GR88200217U & $(19 / 01 / 1990)$ & GR2001200024U & $(31 / 01 / 2002)$ \\
\hline & $\mathrm{Y}$ & 1,658 & GR2000001Y & $(16 / 12 / 1988)$ & GR2002393Y & $(12 / 02 / 2002)$ \\
\hline HK & & 52,380 & HK9376 & $(05 / 03 / 1976)$ & HK1076134 & $(09 / 06 / 2006)$ \\
\hline \multirow[t]{2}{*}{ HR } & & 8,493 & HR920170 & $(11 / 08 / 1994)$ & HR20060152 & $(30 / 06 / 2006)$ \\
\hline & B & 2,596 & HR920188 & $(31 / 12 / 1995)$ & HR20040291 & $(30 / 06 / 2006)$ \\
\hline $\mathrm{HU}$ & & 73,550 & HU2984 & $(02 / 03 / 1970)$ & HU0501031 & $(30 / 01 / 2006)$ \\
\hline
\end{tabular}

Genomics, Society and Policy, Vol.2 No.2 (2006) ISSN: 1746-5354

(C) ESRC Genomics Network. 
GSP Genomics, Society and Policy online 2006, Vol.2, No.2, pp.92-202

\begin{tabular}{|c|c|c|c|c|c|c|}
\hline Country & Code & Documents & Oldest & & Most recent & \\
\hline & $\mathrm{B}$ & 62,121 & HU162185 & $(29 / 01 / 1973)$ & HU224764 & $(30 / 01 / 2006)$ \\
\hline & $\mathrm{U}$ & 3,001 & HU1U & $(28 / 08 / 1992)$ & HU3038U & (30/01/2006) \\
\hline \multirow[t]{2}{*}{ ID } & & 14,348 & ID20298 & $(26 / 11 / 1988)$ & ID30600 & $(03 / 01 / 2002)$ \\
\hline & $\mathrm{B}$ & 238 & ID837 & $(29 / 07 / 1992)$ & ID1055 & $(30 / 10 / 1996)$ \\
\hline \multirow[t]{3}{*}{ IE } & & 15,038 & IE891581 & $(02 / 01 / 1991)$ & IE20050606 & $(22 / 03 / 2006)$ \\
\hline & $\mathrm{B}$ & 37,829 & IE17383 & $(09 / 01 / 1948)$ & IE83868 & $(20 / 04 / 2005)$ \\
\hline & $\mathrm{L}$ & 62,924 & IE10651L & $(03 / 01 / 1930)$ & IE980730L & $(10 / 02 / 1999)$ \\
\hline IL & & 64,355 & IL20106 & $(25 / 01 / 1968)$ & IL168574 & $(18 / 12 / 2005)$ \\
\hline IN & & 54,283 & IN84678 & $(02 / 08 / 1975)$ & IN193030 & $(19 / 06 / 2004)$ \\
\hline IT & & 77,452 & IT354856 & $(11 / 12 / 1937)$ & ITRM20030511 & $(02 / 02 / 2004)$ \\
\hline \multirow[t]{5}{*}{ JP } & & $9,153,235$ & JP46026238 & $(29 / 07 / 1971)$ & JP2006121880 & $(11 / 05 / 2006)$ \\
\hline & $\mathrm{B}$ & $1,486,859$ & JP36008730B & $(26 / 06 / 1961)$ & JP10747284B & $(06 / 05 / 1998)$ \\
\hline & $\mathrm{C}$ & $1,198,487$ & JP746395C & $(23 / 10 / 1974)$ & JP2119385C & $(06 / 12 / 1996)$ \\
\hline & $\mathrm{T}$ & 346,009 & JP54500001T & $(26 / 07 / 1979)$ & JP2006514819T & $(11 / 05 / 2006)$ \\
\hline & $\mathrm{U}$ & $3,721,483$ & JP46000001U & $(13 / 09 / 1971)$ & JP2001000044U & $(26 / 12 / 2001)$ \\
\hline KE & & 1,336 & KE2536 & $(11 / 07 / 1975)$ & KE3892 & $(01 / 09 / 1989)$ \\
\hline \multirow[t]{4}{*}{$\mathrm{KR}$} & & 281,986 & KR7800099 & $(08 / 01 / 1978)$ & KR2005028360 & $(23 / 03 / 2005)$ \\
\hline & $\mathrm{B}$ & 261,391 & KR8000166 & $(10 / 03 / 1980)$ & KR447719 & $(06 / 04 / 2005)$ \\
\hline & $\mathrm{U}$ & 14,734 & KR7800006U & $(10 / 01 / 1978)$ & KR9814477U & $(05 / 06 / 1998)$ \\
\hline & $\mathrm{Y}$ & 198,949 & KR8000075Y & $(28 / 01 / 1980)$ & KR246513Y & $(31 / 10 / 2001)$ \\
\hline \multirow[t]{3}{*}{ LT } & & 2,500 & LT99 & $(25 / 03 / 1994)$ & LT2005063 & $(27 / 12 / 2005)$ \\
\hline & $\mathrm{B}$ & 2,303 & LT3001 & $(25 / 08 / 1994)$ & LT5303 & $(27 / 12 / 2005)$ \\
\hline & $\mathrm{R}$ & 664 & LT2001R & $(15 / 10 / 1992)$ & LT2664R & $(25 / 04 / 1994)$ \\
\hline LU & & 49,784 & LU27693 & $(02 / 06 / 1945)$ & LU91206 & $(30 / 05 / 2006)$ \\
\hline \multirow[t]{2}{*}{ LV } & & 3,655 & LV5001 & $(10 / 06 / 1993)$ & LV12838 & $(20 / 05 / 2002)$ \\
\hline & B & 3,238 & LV10001 & $(20 / 10 / 1994)$ & LV13372 & $(20 / 12 / 2005)$ \\
\hline MC & & 2,570 & $\mathrm{MC} 1$ & $(13 / 12 / 1957)$ & MC200083 & $(07 / 12 / 2005)$ \\
\hline \multirow[t]{3}{*}{ MD } & & 952 & MD930016 & $(30 / 09 / 1994)$ & MD20040196 & $(31 / 05 / 2006)$ \\
\hline & $\mathrm{B}$ & 1,049 & MD1 & $(31 / 01 / 1994)$ & MD3063 & $(31 / 05 / 2006)$ \\
\hline & $\mathrm{F}$ & 1,737 & MD62F & $(30 / 09 / 1994)$ & MD3064F & $(31 / 05 / 2006)$ \\
\hline MN & & 233 & MN57 & $(20 / 11 / 1972)$ & MN465 & $(15 / 06 / 1989)$ \\
\hline MT & & 545 & MT542 & $(25 / 06 / 1968)$ & MT1078 & $(08 / 05 / 1992)$ \\
\hline MW & & 732 & MW3671 & $(09 / 05 / 1973)$ & MW13093 & $(12 / 10 / 1994)$ \\
\hline \multirow[t]{4}{*}{ MX } & & 83,343 & MX143111 & $(14 / 10 / 1980)$ & MXPA99008569 & $(10 / 04 / 2006)$ \\
\hline & B & 11,681 & MX165914 & $(09 / 12 / 1982)$ & MX226742 & $(16 / 03 / 2005)$ \\
\hline & E & 4,770 & MX2938E & $(02 / 01 / 1980)$ & MX7734E & $(27 / 06 / 1991)$ \\
\hline & $\mathrm{Y}$ & 154 & MX8Y & $(09 / 10 / 1992)$ & MX173Y & $(16 / 05 / 1994)$ \\
\hline MY & & 9,618 & MY153 & $(31 / 12 / 1953)$ & MY109280 & $(31 / 12 / 1996)$ \\
\hline \multirow[t]{4}{*}{ NL } & & 316,887 & NL190149 & $(17 / 02 / 1924)$ & NL1030316 & $(18 / 04 / 2006)$ \\
\hline & $\mathrm{B}$ & 51,528 & NL24297B & $(15 / 12 / 1924)$ & NL195034B & $(01 / 08 / 2003)$ \\
\hline & $\mathrm{C}$ & 59,330 & NL90C & $(02 / 01 / 1914)$ & NL1028882C & $(18 / 04 / 2006)$ \\
\hline & $\mathrm{T}$ & 1 & NL8020180T & $(03 / 08 / 1981)$ & NL8020180T & $(03 / 08 / 1981)$ \\
\hline \multirow[t]{3}{*}{$\mathrm{NO}$} & & 148,421 & NO71432 & $(23 / 12 / 1946)$ & NO20055263 & $(12 / 05 / 2006)$ \\
\hline & B & 87,127 & NO155347B & $(08 / 12 / 1966)$ & NO321713B & $(26 / 06 / 2006)$ \\
\hline & $\mathrm{C}$ & 49,845 & NO173699C & $(19 / 01 / 1909)$ & $\mathrm{NO} 174814 \mathrm{C}$ & $(15 / 01 / 2004)$ \\
\hline $\mathrm{NZ}$ & & 85,556 & NZ171180 & $(06 / 03 / 1978)$ & NZ546316 & $(30 / 06 / 2006)$ \\
\hline $\mathrm{OA}$ & & 11,760 & OA1 & $(15 / 01 / 1966)$ & OA11847 & $(23 / 08 / 2005)$ \\
\hline \multirow[t]{2}{*}{$\mathrm{PH}$} & & 19,956 & PH9199 & $(03 / 07 / 1975)$ & PH31699 & $(18 / 01 / 1999)$ \\
\hline & $\mathrm{U}$ & 2,859 & PH5097U & $(02 / 12 / 1981)$ & PH8499U & $(23 / 12 / 1997)$ \\
\hline PL & & 169,703 & PL68374 & (28/02/1973) & PL373762 & $(05 / 09 / 2005)$ \\
\hline
\end{tabular}

Genomics, Society and Policy, Vol.2 No.2 (2006) ISSN: 1746-5354

(C) ESRC Genomics Network. 
GSP Genomics, Society and Policy online 2006, Vol.2, No.2, pp.92-202

\begin{tabular}{|c|c|c|c|c|c|c|}
\hline Country & Code & Documents & Oldest & & Most recent & \\
\hline & $\mathrm{B}$ & 111,678 & PL45687B & $(13 / 03 / 1962)$ & PL191342B & $(28 / 04 / 2006)$ \\
\hline & $\mathrm{U}$ & 11,767 & PL100599U & $(08 / 01 / 1996)$ & PL115371U & $(19 / 09 / 2005)$ \\
\hline & $\mathrm{Y}$ & 8,502 & PL53707Y & $(31 / 01 / 1996)$ & PL62220Y & $(28 / 04 / 2006)$ \\
\hline \multirow[t]{5}{*}{ PT } & & 38,140 & PT56642 & $(01 / 11 / 1971)$ & PT103313 & $(30 / 11 / 2005)$ \\
\hline & $\mathrm{B}$ & 26,143 & PT66476 & $(12 / 03 / 1976)$ & PT102929 & $(30 / 09 / 2005)$ \\
\hline & $\mathrm{T}$ & 26,231 & PT498721T & $(28 / 04 / 2000)$ & PT1580446T & $(30 / 11 / 2005)$ \\
\hline & $\mathrm{U}$ & 2,785 & PT6095U & $(23 / 06 / 1967)$ & PT10046U & $(30 / 11 / 2005)$ \\
\hline & $\mathrm{Y}$ & 1,491 & PT6095Y & $(13 / 02 / 1969)$ & PT9951Y & $(31 / 05 / 2005)$ \\
\hline \multirow[t]{2}{*}{ RO } & & 38,589 & RO1 & $(08 / 01 / 1907)$ & RO102718 & $(22 / 03 / 1993)$ \\
\hline & $\mathrm{B}$ & 18,404 & RO102338 & $(20 / 08 / 1991)$ & RO120682 & $(30 / 05 / 2006)$ \\
\hline RUC & & 272,924 & RU2000001 & $(15 / 02 / 1993)$ & RU2274965 & $(20 / 04 / 2006)$ \\
\hline \multirow[t]{3}{*}{ SE } & & 152,321 & SE122183 & $(13 / 07 / 1948)$ & SE0501863 & $(27 / 06 / 2006)$ \\
\hline & $\mathrm{B}$ & 170,658 & SE205247 & $(07 / 06 / 1966)$ & SE470607 & $(17 / 04 / 2000)$ \\
\hline & $\mathrm{C}$ & 126,609 & SE3819 & $(30 / 09 / 1892)$ & SE527871 & $(27 / 06 / 2006)$ \\
\hline \multirow[t]{2}{*}{ SG } & & 27,341 & SG39090 & $(17 / 08 / 1990)$ & SG118232 & $(27 / 06 / 2006)$ \\
\hline & G & 10,238 & SG1082G & $(25 / 02 / 1983)$ & SG37095G & $(01 / 09 / 1995)$ \\
\hline \multirow[t]{3}{*}{ SI } & & 5,731 & SI9100001 & $(27 / 11 / 1992)$ & SI21869 & $(28 / 02 / 2006)$ \\
\hline & $\mathrm{B}$ & 572 & SI8111622 & $(30 / 06 / 1998)$ & SI9720005 & $(28 / 02 / 2006)$ \\
\hline & $\mathrm{T}$ & 5,778 & SI619406T & $(31 / 10 / 1997)$ & SI1525420T & $(28 / 02 / 2006)$ \\
\hline \multirow[t]{2}{*}{ SK } & & 20,875 & SK3893 & $(07 / 07 / 1993)$ & SK51112005 & $(01 / 06 / 2006)$ \\
\hline & $\mathrm{B}$ & 7,441 & SK277680B & $(08 / 12 / 1993)$ & SK285127B & $(01 / 06 / 2006)$ \\
\hline SU & & $1,162,205$ & SU18207 & $(30 / 09 / 1930)$ & SU1829367 & $(20 / 04 / 2006)$ \\
\hline $\mathrm{TJ}$ & & 164 & TJ96000415 & $(14 / 11 / 1998)$ & TJ20040823 & $(06 / 01 / 2006)$ \\
\hline \multirow[t]{3}{*}{ TR } & & 17,145 & TR16570 & $(01 / 01 / 1973)$ & TR200503829 & $(21 / 04 / 2006)$ \\
\hline & $\mathrm{T}$ & 12,532 & TR970231T & $(21 / 03 / 1997)$ & TR200505223T & $(21 / 04 / 2006)$ \\
\hline & $\mathrm{U}$ & 6,805 & TR960121U & $(21 / 06 / 1996)$ & TR200601427U & $(21 / 04 / 2006)$ \\
\hline \multirow[t]{2}{*}{ TWB } & & 115,969 & TW211035B & $(11 / 08 / 1993)$ & TW235029B & $(21 / 06 / 2005)$ \\
\hline & $\mathrm{Y}$ & 113,084 & TW378487Y & $(01 / 01 / 2000)$ & TW269707Y & $(01 / 07 / 2005)$ \\
\hline \multirow[t]{6}{*}{ US } & & $5,986,161$ & US11713 & $(19 / 09 / 1854)$ & US2006143744 & $(29 / 06 / 2006)$ \\
\hline & $\mathrm{B}$ & 905,282 & US3367320 & $(29 / 12 / 1981)$ & US7073200 & $(04 / 07 / 2006)$ \\
\hline & E & 12,611 & USRE892E & $(07 / 02 / 1860)$ & USRE39157E & $(04 / 07 / 2006)$ \\
\hline & $\mathrm{H}$ & 2,122 & USH1H & $(03 / 12 / 1985)$ & USH2162H & $(04 / 07 / 2006)$ \\
\hline & $\mathrm{P}$ & 13,236 & US3987P & $(14 / 12 / 1976)$ & US16774P & $(04 / 07 / 2006)$ \\
\hline & S & 87,863 & USD65880S & $(28 / 10 / 1924)$ & USD524510S & $(04 / 07 / 2006)$ \\
\hline \multirow[t]{2}{*}{$\mathrm{VN}$} & & 112 & VN1 & $(06 / 07 / 1984)$ & VN344 & $(25 / 04 / 1997)$ \\
\hline & $\mathrm{U}$ & 52 & VN1U & $(24 / 09 / 1989)$ & VN167U & $(25 / 10 / 1996)$ \\
\hline \multirow[t]{2}{*}{ WO } & & $1,140,656$ & WO7800001 & $(19 / 10 / 1978)$ & WO2006072117 & $(06 / 07 / 2006)$ \\
\hline & $\mathrm{B}$ & 8,329 & WO9817166 & $(01 / 10 / 1998)$ & WO2006056260 & $(06 / 07 / 2006)$ \\
\hline $\mathrm{ZA}$ & & 206,516 & ZA6901534 & $(27 / 01 / 1971)$ & ZA200501578 & $(09 / 12 / 2005)$ \\
\hline DZ & & 16 & DZ2403 & $(04 / 01 / 2005)$ & DZ2783 & $(08 / 10 / 2005)$ \\
\hline $\mathrm{GC}$ & & 77 & GC0000093 & $(29 / 06 / 2005)$ & GC0000170 & $(29 / 06 / 2005)$ \\
\hline MA & & 797 & MA27120 & $(03 / 01 / 2005)$ & MA27916 & $(02 / 05 / 2006)$ \\
\hline \multirow[t]{3}{*}{ UA } & & 290 & UA72070 & $(17 / 01 / 2005)$ & UA74127 & $(17 / 10 / 2005)$ \\
\hline & $\mathrm{C}$ & 760 & UA36210 & $(17 / 01 / 2005)$ & UA74120 & $(17 / 10 / 2005)$ \\
\hline & $\mathrm{U}$ & 4,065 & UA6429U & $(17 / 01 / 2005)$ & UA10058U & $(17 / 10 / 2005)$ \\
\hline & & $50,874,779$ & & & & \\
\hline
\end{tabular}




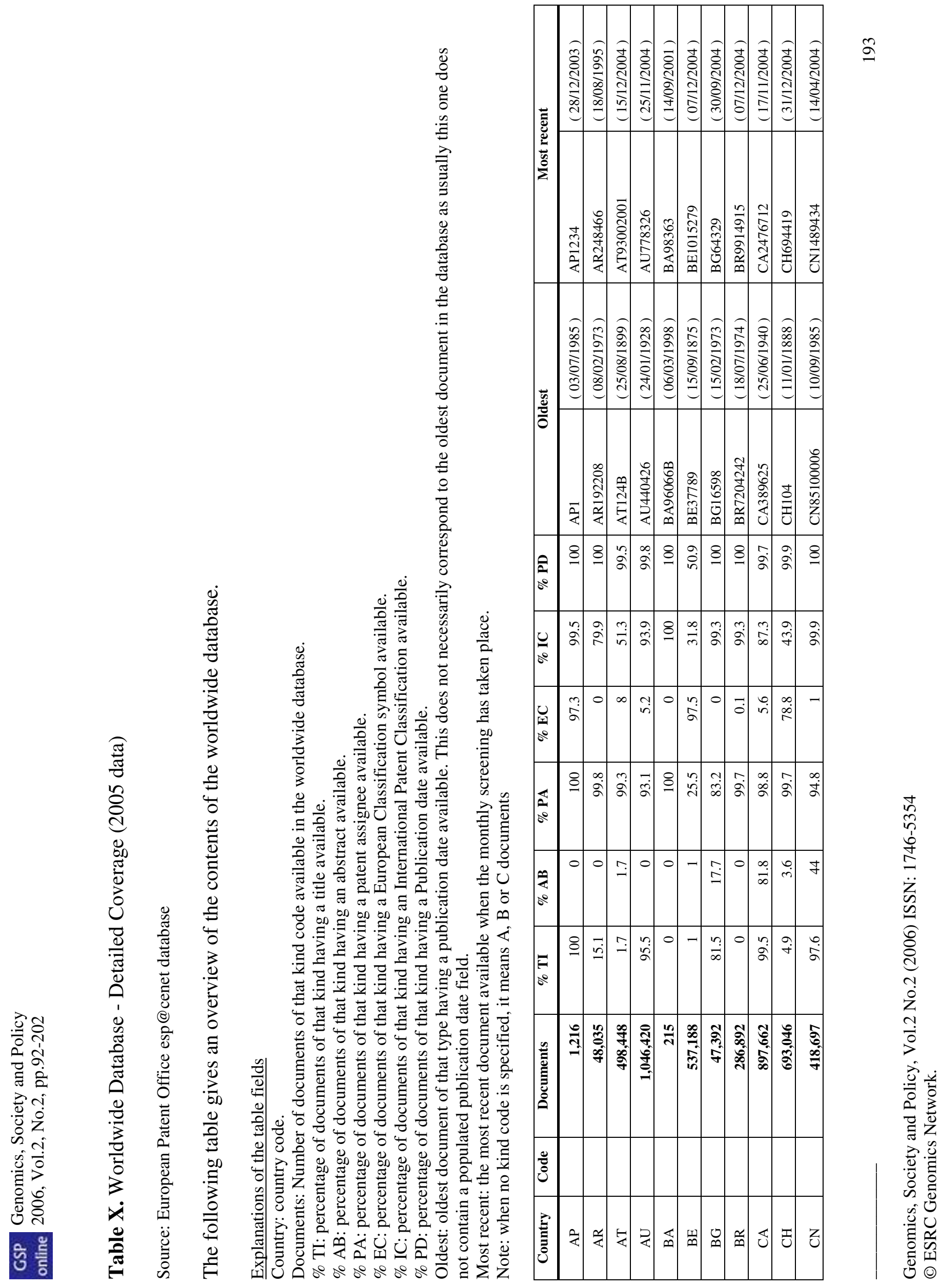




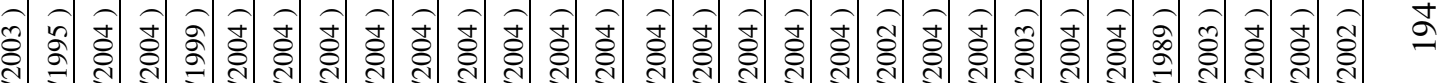

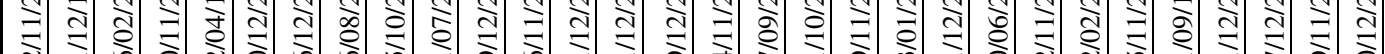

(1)

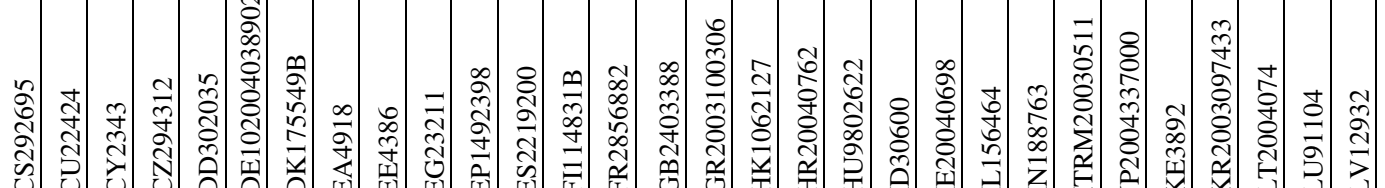

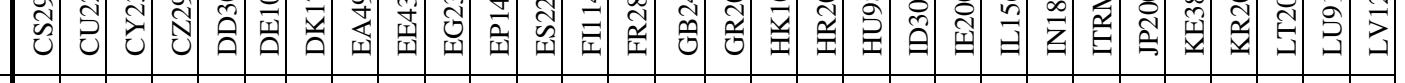

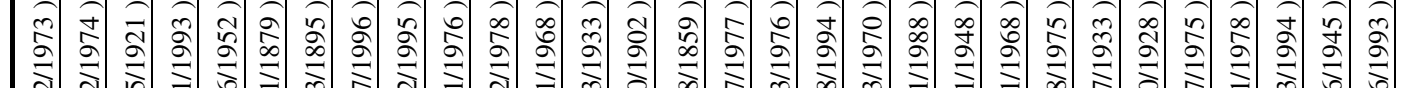

离

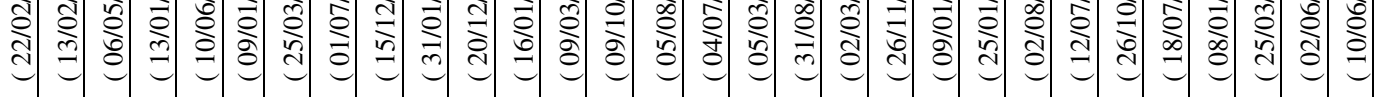

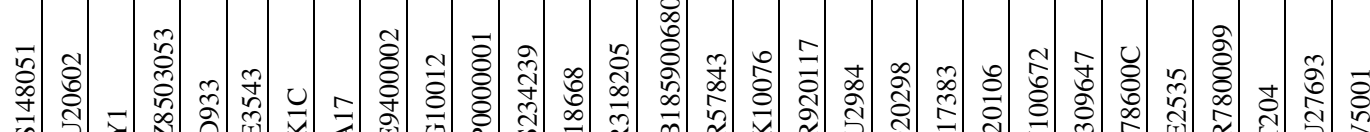

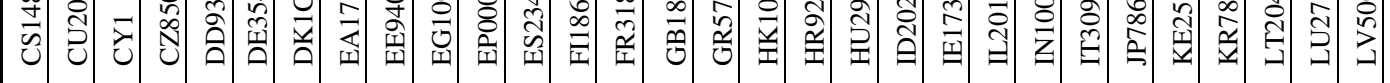

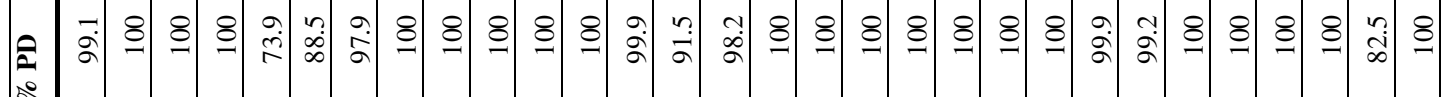
$\therefore$

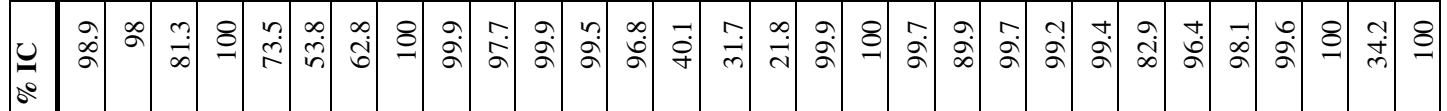

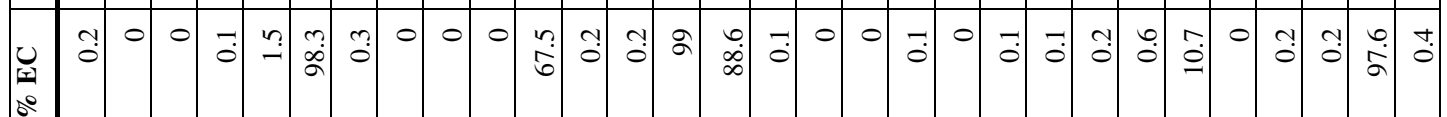
๘ œ $\circ$

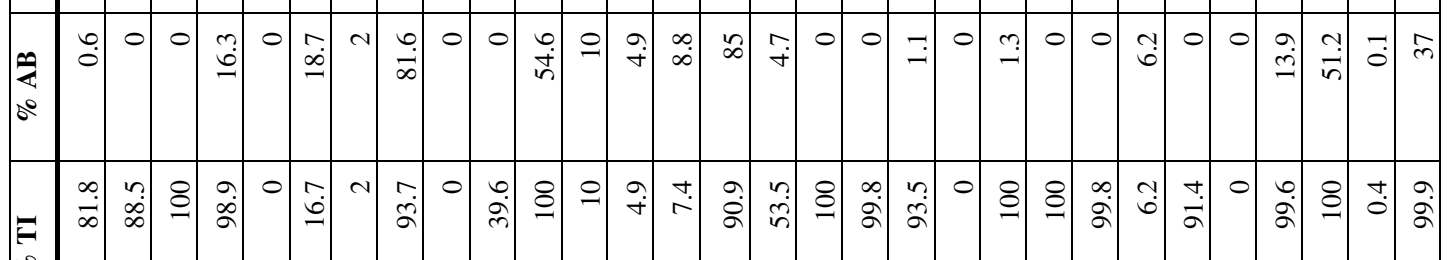
-

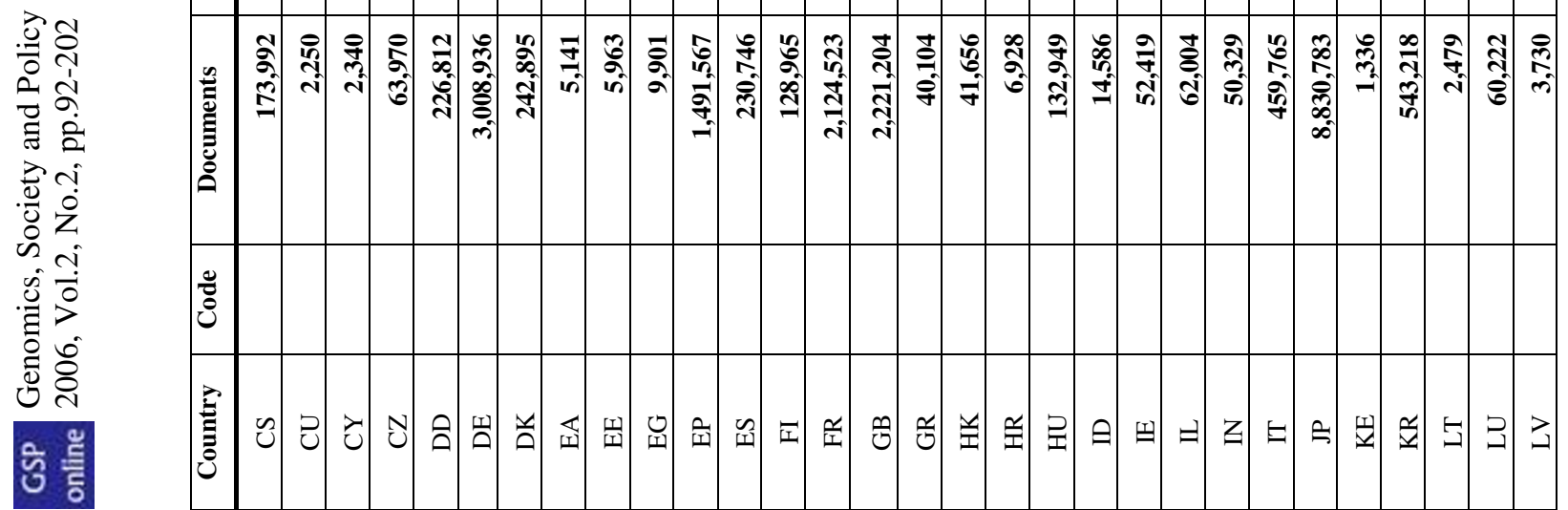




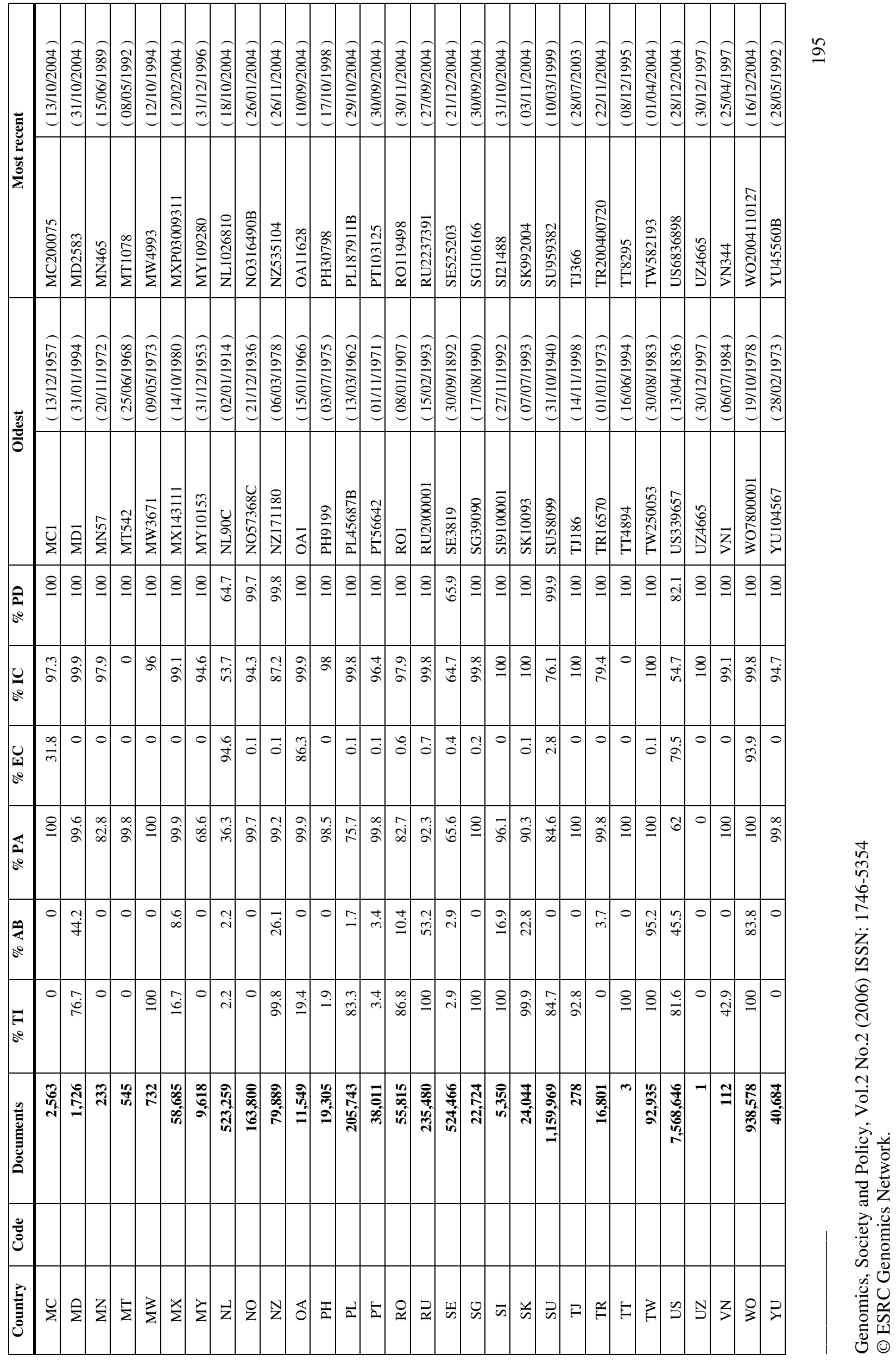




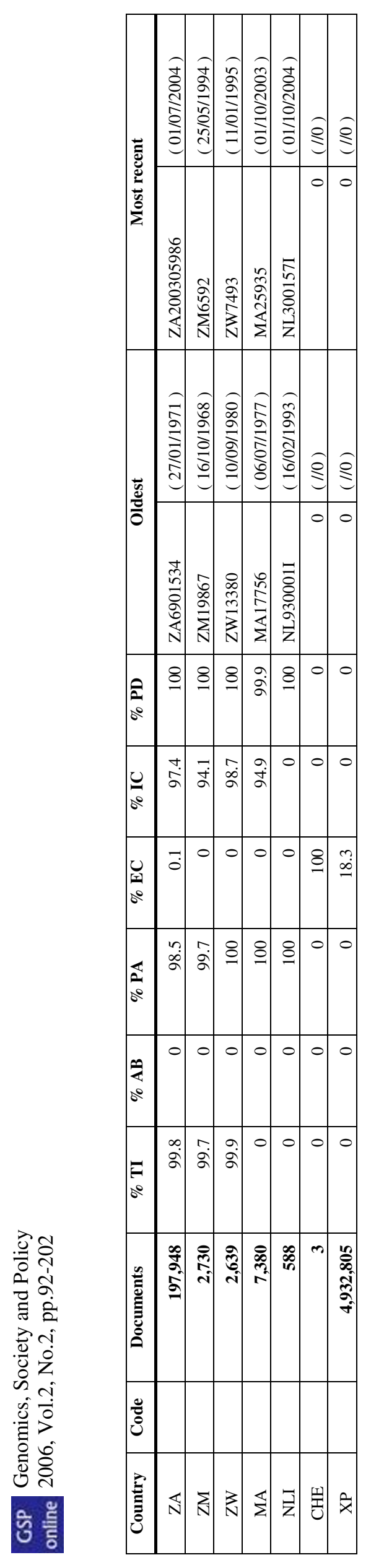


Table XI. Worldwide Database - Detailed Coverage (2004 data)

Source: European Patent Office esp@cenet database

Note: Taken from hard copy.

The following table gives an overview of the contents of the worldwide database

Explanations of the table fields

Country: country code.

Code: kind code.

Documents: Number of documents of that kind code available in the worldwide database.

Oldest: oldest document of that type having a publication date available. This does not necessarily correspond to the oldest document in the database as usually this one does not contain a populated publication date field.

Most recent: the most recent document available when the monthly screening has taken place.

Note: when no kind code is specified, it means A, B or C documents

http://ep.espacenet.com/help?topic=index\&locale=en_EP\&method=handleHelpTopic.

Table is current as of 2 August 2004

\begin{tabular}{|c|c|}
\hline Country & 2,004 \\
\hline $\mathrm{AP}$ & 1,165 \\
\hline APD & 3 \\
\hline APU & 1 \\
\hline $\mathrm{AR}$ & 40,755 \\
\hline AT & 497,623 \\
\hline $\mathrm{AT}$ & 271,247 \\
\hline ATT & 7,026 \\
\hline $\mathrm{AU}$ & $1,042,670$ \\
\hline AUD & \\
\hline BA & 215 \\
\hline BAD & 1 \\
\hline $\mathrm{BE}$ & 536,719 \\
\hline BET & 102 \\
\hline BG & 47,043 \\
\hline BGU & 966 \\
\hline BGY & 679 \\
\hline BR & 278,133 \\
\hline BRU & 68,978 \\
\hline BRY & 1 \\
\hline CA & 833,947 \\
\hline CAE & 54 \\
\hline $\mathrm{CAF}$ & 5 \\
\hline $\mathrm{CH}$ & 692,770 \\
\hline CHD & 6,919 \\
\hline $\mathrm{CHH}$ & 8 \\
\hline $\mathrm{CN}$ & 416,294 \\
\hline CNT & 73,982 \\
\hline $\mathrm{CNU}$ & 599,219 \\
\hline $\mathrm{CNY}$ & 7,834 \\
\hline CS & 173,992 \\
\hline $\mathrm{CU}$ & 2,250 \\
\hline $\mathrm{CY}$ & 2,302 \\
\hline $\mathrm{CZ}$ & 62,931 \\
\hline
\end{tabular}


GSP Genomics, Society and Policy online 2006, Vol.2, No.2, pp.92-202

\begin{tabular}{|c|c|}
\hline Country & 2,004 \\
\hline CZU & 5,100 \\
\hline DD & 226,810 \\
\hline DDT & 1 \\
\hline DDU & 5,634 \\
\hline DE & $2,990,574$ \\
\hline DED & 656,420 \\
\hline DEI & 252 \\
\hline DET & 333,739 \\
\hline DK & 241,104 \\
\hline DKL & 1 \\
\hline DKT & 61,226 \\
\hline DKU & 4,566 \\
\hline DKY & 85 \\
\hline EA & 4,740 \\
\hline $\mathrm{EE}$ & 5,811 \\
\hline EG & 9,829 \\
\hline EP & $1,440,769$ \\
\hline ES & 230,082 \\
\hline EST & 165,065 \\
\hline ESU & 127,152 \\
\hline ESY & 197,345 \\
\hline FA & 1 \\
\hline FI & 128,358 \\
\hline FIU & 6,126 \\
\hline FR & $2,117,810$ \\
\hline FRE & 94,192 \\
\hline FRF & 329 \\
\hline FRM & 8,091 \\
\hline GB & $2,215,342$ \\
\hline GR & 39,964 \\
\hline GRT & 28,128 \\
\hline GRU & 1,216 \\
\hline GRY & 1,658 \\
\hline $\mathrm{HK}$ & 40,009 \\
\hline HR & 6,634 \\
\hline $\mathrm{HU}$ & 131,960 \\
\hline HUU & 2,737 \\
\hline ID & 14,586 \\
\hline IDS & 53 \\
\hline IE & 52,240 \\
\hline IEL & 62,936 \\
\hline $\mathrm{IL}$ & 61,412 \\
\hline IN & 50,329 \\
\hline INE & 3 \\
\hline IT & 459,758 \\
\hline ITT & 2 \\
\hline ITU & 35,435 \\
\hline ITY & 24,928 \\
\hline ITZ & 20,020 \\
\hline JP & $8,661,837$ \\
\hline JPB1 & 979 \\
\hline JPB2 & 17,800 \\
\hline
\end{tabular}


GSP Genomics, Society and Policy online 2006, Vol.2, No.2, pp.92-202

\begin{tabular}{|c|c|}
\hline Country & 2,004 \\
\hline JPT & 271,623 \\
\hline JPU & $3,712,670$ \\
\hline JPY & 98,756 \\
\hline JPZ & 6 \\
\hline KE & 1,336 \\
\hline KED & 16 \\
\hline $\mathrm{KR}$ & 543,217 \\
\hline KRU & 14,737 \\
\hline KRY & 198,943 \\
\hline LT & 2,440 \\
\hline LTR & 664 \\
\hline LU & 60,191 \\
\hline LUD & 15 \\
\hline LV & 3,730 \\
\hline $\mathrm{MC}$ & 2,560 \\
\hline MCE & 97 \\
\hline $\mathrm{MD}$ & 1,652 \\
\hline MDE & 89 \\
\hline MDF & 1,344 \\
\hline MDG & 1 \\
\hline MDU & 21 \\
\hline MDW & 77 \\
\hline MDY & 16 \\
\hline MN & 233 \\
\hline MT & 545 \\
\hline MW & 732 \\
\hline MX & 48,858 \\
\hline MXE & 4,770 \\
\hline MXY & 154 \\
\hline MY & 9,618 \\
\hline MY-U & 1 \\
\hline $\mathrm{NC}$ & 46 \\
\hline NL & 521,572 \\
\hline NLT & 1,555 \\
\hline NO & 163,800 \\
\hline NZ & 78,029 \\
\hline $\mathrm{OA}$ & 11,445 \\
\hline OAE & 153 \\
\hline $\mathrm{PH}$ & 18,947 \\
\hline PHU & 2,859 \\
\hline PL & 202,263 \\
\hline PLU & 10,907 \\
\hline PLY & 6,832 \\
\hline PT & 37,961 \\
\hline PTD & 2 \\
\hline PTT & 17,928 \\
\hline PTU & 2,663 \\
\hline PTY & 1,432 \\
\hline RO & 55,589 \\
\hline RUC & 227,862 \\
\hline SE & 522,740 \\
\hline SEE & 26 \\
\hline
\end{tabular}


GSP Genomics, Society and Policy online 2006, Vol.2, No.2, pp.92-202

\begin{tabular}{|l|r|}
\hline Country & 2,004 \\
\hline SEL & 2 \\
SG & 21,980 \\
SGG & 10,238 \\
SI & 5,258 \\
SIT & 3,976 \\
SK & 23,768 \\
SU & $1,159,629$ \\
SUT & 8 \\
TH & 3 \\
TJ & 278 \\
TJR & 30 \\
TR & 16,668 \\
TRT & 11,100 \\
TRU & 3,593 \\
TRY & 1 \\
TT & 3 \\
TW & 87,967 \\
TWY & 75,699 \\
TWB & 87,967 \\
US & $7,384,459$ \\
USE & 23,313 \\
USF & 46 \\
USH & 2,079 \\
USI & 4,170 \\
USP & 12,092 \\
USR & 1 \\
USS & 56,984 \\
UZ & 1 \\
VN & 112 \\
VNU & 52 \\
WO & 892,902 \\
YU & 40,684 \\
YUD & 1 \\
ZA & 194,826 \\
ZAD & 197 \\
ZM & 2,730 \\
ZMD & $2,675,154$ \\
ZW & 2,639 \\
MA & 7,380 \\
NLI & \\
XP & \\
& \\
\hline
\end{tabular}




\section{Table XII. Country codes}

Source: http://ep.espacenet.com/help?locale=en_EP\&method=handleHelpTopic\&topic=countrycodes.

Country codes are two letters indicating the country or organisation where the patent application was filed or granted (e.g. GB for Great Britain).

The list of countries covered by our worldwide database is shown in this table:

\begin{tabular}{|c|c|}
\hline Code & Name \\
\hline $\mathrm{AP}$ & African Regional Industrial Property Organization \\
\hline AR & Argentina \\
\hline AT & Austria \\
\hline AU & Australia \\
\hline $\mathrm{BA}$ & Bosnia and Herzegovina \\
\hline $\mathrm{BE}$ & Belgium \\
\hline $\mathrm{BG}$ & Bulgaria \\
\hline $\mathrm{BR}$ & Brazil \\
\hline $\mathrm{CA}$ & Canada \\
\hline $\mathrm{CH}$ & Switzerland \\
\hline $\mathrm{CN}$ & China \\
\hline $\mathrm{CS}$ & Czechoslovakia (up to 1993) \\
\hline $\mathrm{CU}$ & Cuba \\
\hline $\mathrm{CY}$ & Cyprus \\
\hline $\mathrm{CZ}$ & Czech Republic \\
\hline DD & Germany, excluding the territory that, prior to 3 October 1990, constituted the Federal Republic of Germany \\
\hline $\mathrm{DE}$ & Germany \\
\hline DK & Denmark \\
\hline DZ & Algeria \\
\hline EA & Eurasian Patent Organisation \\
\hline EE & Estonia \\
\hline EG & Egypt \\
\hline EP & European Patent Office \\
\hline ES & Spain \\
\hline FI & Finland \\
\hline FR & France \\
\hline GB & United Kingdom \\
\hline GR & Greece \\
\hline HK & Hong Kong \\
\hline HR & Croatia \\
\hline $\mathrm{HU}$ & Hungary \\
\hline $\mathrm{IE}$ & Ireland \\
\hline $\mathrm{IL}$ & Israel \\
\hline IN & India \\
\hline IT & Italy \\
\hline JP & Japan \\
\hline $\mathrm{KE}$ & Kenya \\
\hline $\mathrm{KR}$ & Republic of Korea \\
\hline LT & Lithuania \\
\hline $\mathrm{LU}$ & Luxembourg \\
\hline LV & Latvia \\
\hline
\end{tabular}




\begin{tabular}{|c|c|}
\hline Code & Name \\
\hline $\mathrm{MC}$ & Monaco \\
\hline MD & Republic of Moldova \\
\hline $\mathrm{MN}$ & Mongolia \\
\hline MT & Malta \\
\hline MW & Malawi \\
\hline MX & Mexico \\
\hline MY & Malaysia \\
\hline $\mathrm{NC}$ & New Caledonia \\
\hline NL & Netherlands \\
\hline NO & Norway \\
\hline $\mathrm{NZ}$ & New Zealand \\
\hline $\mathrm{OA}$ & African Intellectual Property Organization \\
\hline $\mathrm{PH}$ & Philippines \\
\hline PL & Poland \\
\hline $\mathrm{PT}$ & Portugal \\
\hline RO & Romania \\
\hline RU & Russian Federation \\
\hline SE & Sweden \\
\hline SG & Singapore \\
\hline SI & Slovenia \\
\hline SK & Slovakia \\
\hline SU & Union of Soviet Socialist Republics (USSR) \\
\hline $\mathrm{TJ}$ & Tajikistan \\
\hline $\mathrm{TR}$ & Turkey \\
\hline TT & Trinidad and Tobago \\
\hline TW & Taiwan \\
\hline US & United States of America \\
\hline VN & Vietnam \\
\hline WO & World Intellectual Property Organisation (WIPO) \\
\hline YU & Yugoslavia \\
\hline $\mathrm{ZA}$ & South Africa \\
\hline $\mathrm{ZM}$ & Zambia \\
\hline ZW & Zimbabwe \\
\hline
\end{tabular}

